DOE/D-10430(NV)

\title{
U.S. Hydropower Resource Assessment for Nevada
}

\author{
Prepared by: \\ Alison M. Conner \\ James E. Francfort \\ Project Manager: \\ Ben N. Rinehart
}

Published October 1997

Idaho National Engineering and Environmental Laboratory Renewable Energy Products Department Lockheed Martin Idaho Technologies Company Idaho Falls, Idaho 83415

\section{Prepared for the}

U.S. Department of Energy

Assistant Secretary for Energy Efficiency and Renewable Energy

Under DOE Idaho Operations Office

Contract DE-AC07-94ID13223

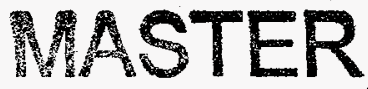




\begin{abstract}
The U.S. Department of Energy is developing an estimate of the undeveloped hydropower potential in the United States. The Hydropower Evaluation Software (HES) is a computer model that was developed by the Idaho National Engineering Laboratory ${ }^{a}$ for this purpose. HES measures the undeveloped hydropower resources available in the United States, using uniform criteria for measurement. The software was developed and tested using hydropower information and data provided by the Southwestern Power Administration. It is a menu-driven program that allows the personal computer user to assign environmental attributes to potential hydropower sites, calculate development suitability factors for each site based on the environmental attributes present, and generate reports based on these suitability factors. This report describes the resource assessment results for the State of Nevada.
\end{abstract}

a. In January 1997, the name of the Idaho National Engineering Laboratory (INEL) was changed to the Idaho National Engineering and Environmental Laboratory (INEEL). INEEL will be used throughout the text of the document, except where the use of INEL is historically important. 


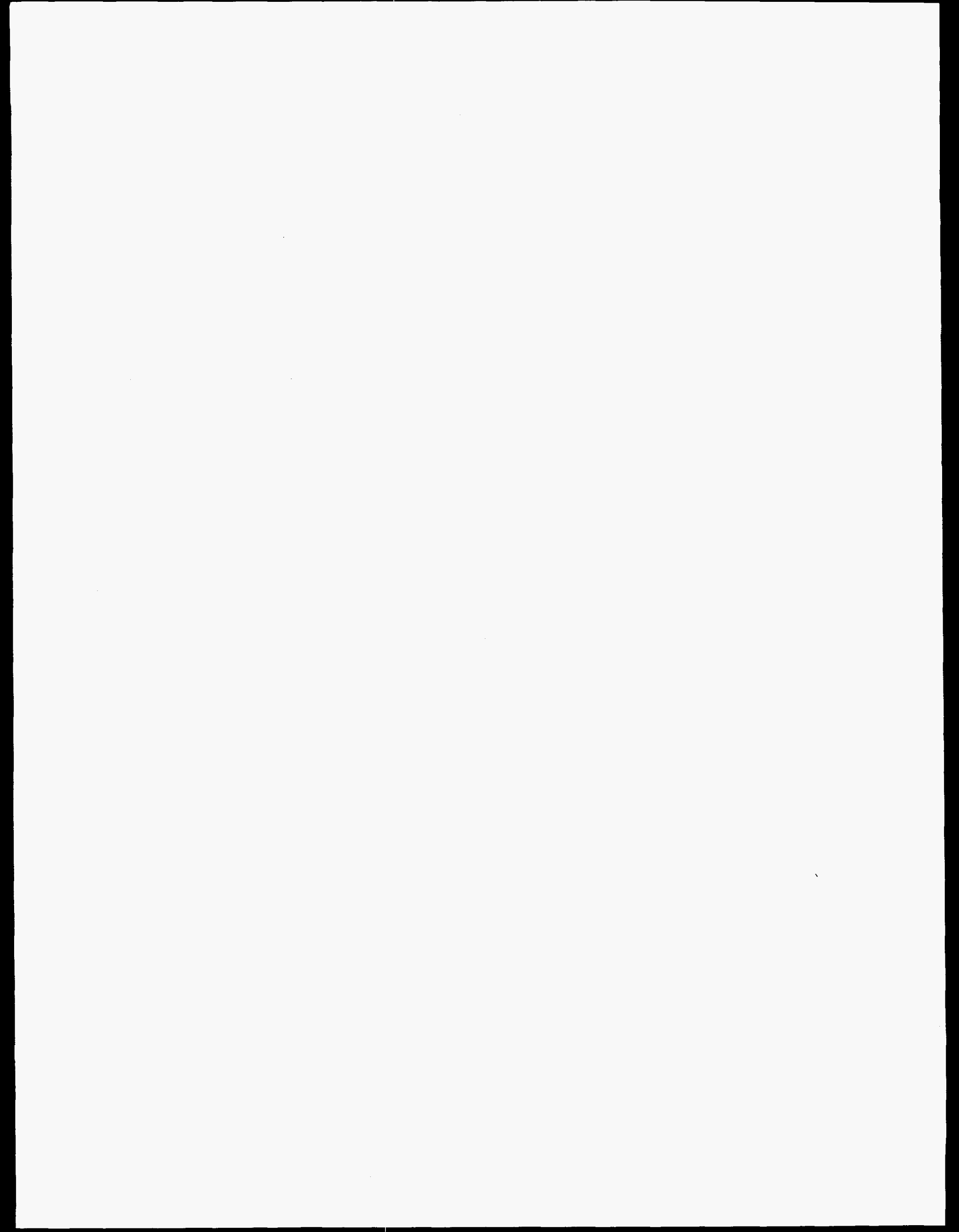




\section{ACKNOWLEDGMENTS}

The authors thank Peggy A. M. Brookshier and John V. Flynn of the U.S. Department of Energy, and Michael J. Anderson of the State of Nevada for their active participation and timely comments. 


\section{CONTENTS}

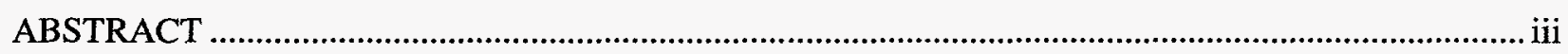

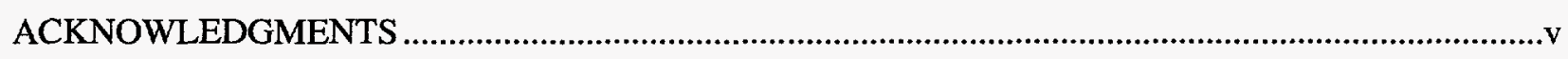

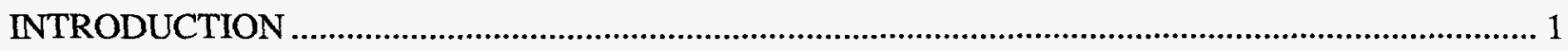

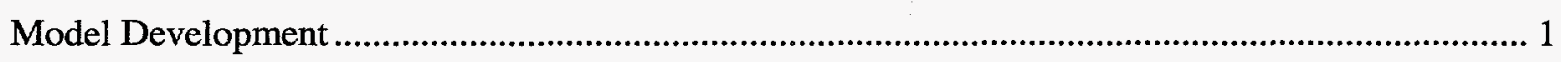

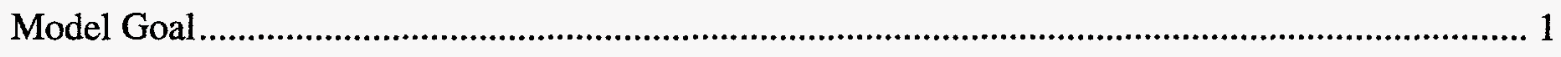

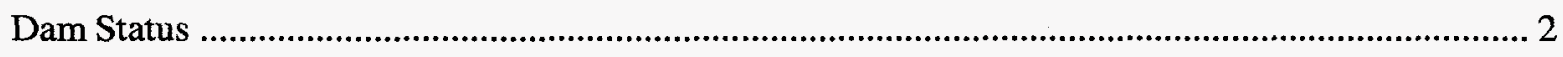

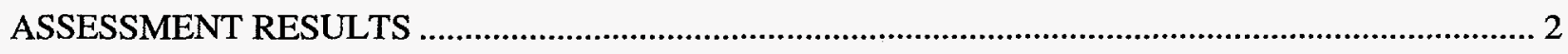

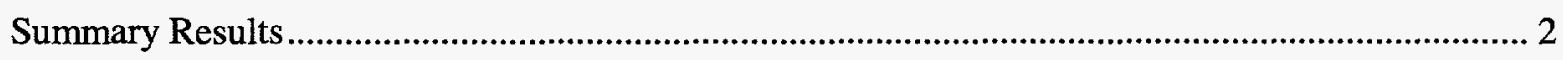

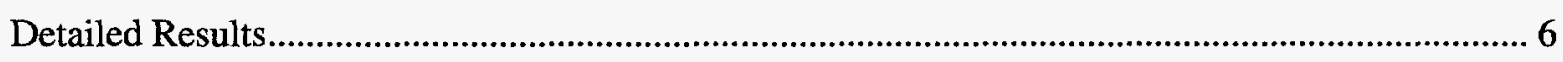

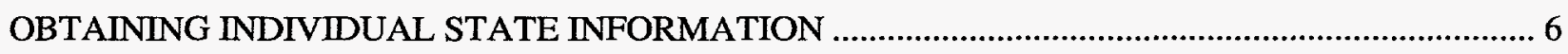

ADDITIONAL HYDROPOWER EVALUATION SOFTWARE INFORMATION .............................. 7

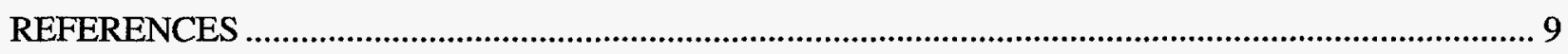

Appendix A-Summary Report

Appendix B-River Basins Report

Appendix C-Nevada Sites List

Appendix D-Individual Resource Database List

Appendix E-List of 201 Additional Sites

\section{FIGURES}

1. Number of sites, by capacity groups, with HES-modeled undeveloped hydropower potential.......... 3

2. The nonmodeled and HES-modeled undeveloped hydropower potential ...................................... 3 
3. The number of sites with undeveloped hydropower potential and the total megawatts of HES-modeled undeveloped hydropower potential.................................................................. 4

4. The South Fork Dam is an example of an earthfilled embankment dam ...................................... 4

5. An aerial view of the South Fork Dam on the South Fork Humbolt River, Nevada.......................... 5

6. Number of sites with undeveloped hydropower potential in the Nevada river basins...................... 5

7. Megawatts of HES-modeled undeveloped hydropower potential in the Nevada river basins ............ 6

\section{TABLES}

1. Undeveloped hydropower potential summary for Nevada 


\section{U.S. Hydropower Resource Assessment for Nevada}

\section{INTRODUCTION}

In June 1989, the U.S. Department of Energy initiated the development of a National Energy Strategy to identify the energy resources available to support the expanding demand for energy in the United States. Public hearings conducted as part of the strategy development process indicated that undeveloped hydropower resources were not well defined. As a result, the Department of Energy established an interagency Hydropower Resource Assessment Team to ascertain the undeveloped hydropower potential. In connection with these efforts by the Department of Energy, the Idaho National Engineering Laboratory designed the Hydropower Evaluation Software (HES), which has been used to perform a resource assessment of the undeveloped conventional hydropower potential in over 30 states. This report presents the results of the hydropower resource assessment for the State of Nevada. Undeveloped pumped storage hydropower potential is not included.

The HES was developed as a tool to measure undeveloped hydropower potential regionally or by state. The software is not intended to provide precise development factors for individual sites, but to provide regional or state totals. Because the software was developed as a generic measurement tool encompassing national issues, regional and state totals must be considered judiciously; various local issues may skew undeveloped hydropower potential totals. The information for the resource assessment was compiled from the Federal Energy Regulatory Commission's Hydroelectric Power Resources Assessment database and several other sources. Refer to DOE/ID-10338, the User's Manual (Francfort, Matthews, Rinehart 1991) for the specifics of the software and to DOE/ID-10430.1, the Status Report (Conner, Francfort, Rinehart 1996) for an overview of all resource assessment activities to date.

\section{Model Development}

Hydropower Evaluation Software, both a probability-factor computer model and a database, is a menu-driven program that is intended to be user-friendly. Computer screens and report-generation capabilities were developed to meet the needs of users nationwide. The software uses environmental attribute data to generate an overall project environmental suitability factor (PESF) between 0.1 and 0.9 , where 0.9 indicates the highest likelihood of development and 0.1 indicates the lowest likelihood of development. The suitability factors depend on the unique environmental attributes of each potential site. They reflect the considerations that (a) environmental concerns can make a potential site unacceptable, prohibiting its development (for a suitability factor of 0.1 ), or (b) if there are no environmental concerns, there is no negative effect on the likelihood of site development (for a suitability factor of 0.9 ). A combination of attributes can result in a lower suitability factor because multiple environmental considerations would reduce the likelihood that a site may be developed to its physical potential.

\section{Model Goal}

The goal of the HES is to assemble an accurate resource database of all sites with undeveloped hydropower potential in the United States for use as a planning tool to determine the viable national hydropower potential. Undeveloped hydropower potential is not limited to the development of new sites; it also includes the development of additional hydropowergenerating capacity at sites that currently have hydropower, but are not developed to their full potential. This undeveloped hydropower potential is a source of nonpolluting, renewable energy available to meet the growing power needs of the United States. The HES should help make this goal obtainable and ensure a set of uniform criteria for national assessment. 


\section{Dam Status}

The effects of environmental attributes vary by dam status. The dam status classifications used are as follows:

$\mathrm{W}=$ Developed hydropower site with current power generation, but the total hydropower potential has not been fully developed. Only the undeveloped hydropower potential is discussed in this report.

$\mathrm{W} / \mathrm{O}=$ Developed site without current power generation. The site has some type of developed impoundment or diversion structure, but no developed hydropower generating capability.

$\mathrm{U}=$ Undeveloped site. The site does not have power generation capability nor a developed impoundment or diversion structure.

\section{ASSESSMENT RESULTS Summary Results}

A total of 181 sites (Table 1) have been identified and assessed for their undeveloped hydropower potential. The HES results for individual site capacities range from 9 kilowatts (kW) to 3 megawatts (MW). All of the HESmodeled sites in Nevada are small hydropower sites (no individual site is larger than $10 \mathrm{MW}$ ). In fact, the majority, or $87 \%$, of the sites are less than $1 \mathrm{MW}$ each (Figure 1).

The nonmodeled undeveloped hydropower potential total for Nevada was identified as 126
MW. The HES results lowers this estimate about $47 \%$ to $67 \mathrm{MW}$. The greatest reduction in undeveloped hydropower potential, by MW, occurs at sites with no current power generation capability nor impoundment or diversion structure in place (undeveloped category [U]). These sites have an HES-modeled undeveloped hydropower potential of $32 \mathrm{MW}$, a $60 \%$ or 48 MW reduction in the estimated undeveloped hydropower potential (Figure 2). Figure 3 correlates the number of sites that have undeveloped hydropower potential with the total megawatts of HES-modeled undeveloped hydropower potential. Figures 4 and 5 are photographs of the South Fork Dam on the South Fork Humbolt River. Owned by the State of Nevada, the South Fork Dam reservoir has a 30,000 acre feet (AF) storage capacity and is not equipped for power generation.

The 181 identified sites are located within 18 major river basins and 9 lake-based river basins. The number of sites per river basin ranges from 1 in the Big Lake, Gridley Lake, Hot Creek, Middlegate, Newark, Pueblo, and Railroad river basins to 38 in the Humbolt River Basin (Figure 6). The Humbolt River Basin has the most undeveloped hydropower potential (16 MW) of the Nevada river basins. Twenty-four percent of the undeveloped hydropower potential in the State of Nevada is contained within the Humbolt River Basin (Figure 7).

The State of Nevada also provided information on an additional 201 hydropower sites (Appendix E). These sites do not have any undeveloped hydropower potential; therefore, they were not included in the HES model.

Table 1. Undeveloped hydropower potential summary for Nevada. The table contains the nonmodeled undeveloped nameplate potential and the HES-modeled undeveloped potential totals.

\begin{tabular}{lccc}
\hline & Number of projects & $\begin{array}{c}\text { Nameplate potential } \\
(\mathrm{MW})\end{array}$ & $\begin{array}{c}\text { HES-modeled potential } \\
(\mathrm{MW})\end{array}$ \\
\cline { 2 - 4 } With Power & 9 & 4.7 & 3.9 \\
W/O Power & 48 & 41.2 & 31.1 \\
Undeveloped & 124 & 80.0 & 32.2 \\
State Total & 181 & 125.9 & 67.2 \\
\hline
\end{tabular}




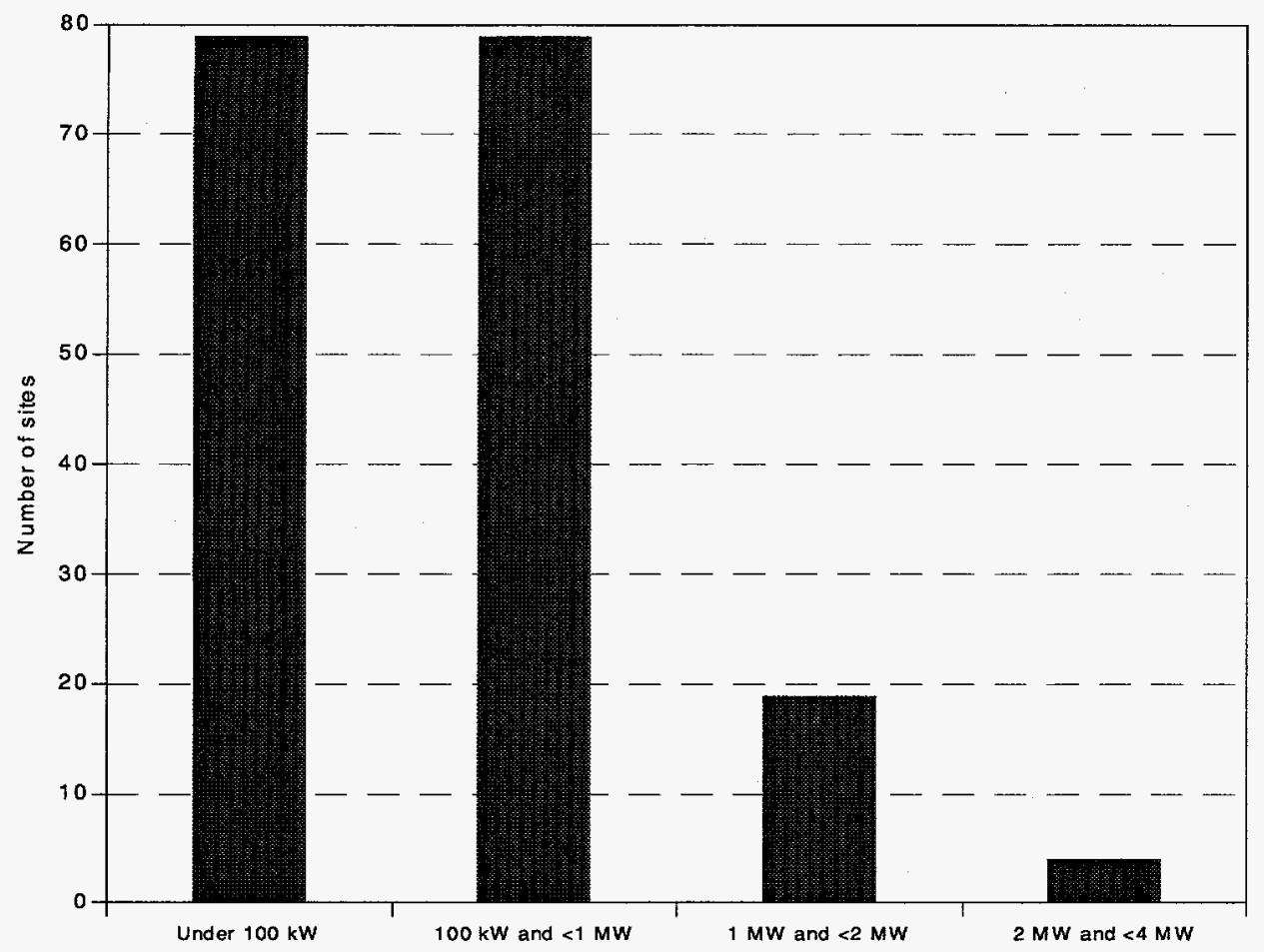

Figure 1. Number of sites, by capacity groups, with HES-modeled undeveloped hydropower potential.

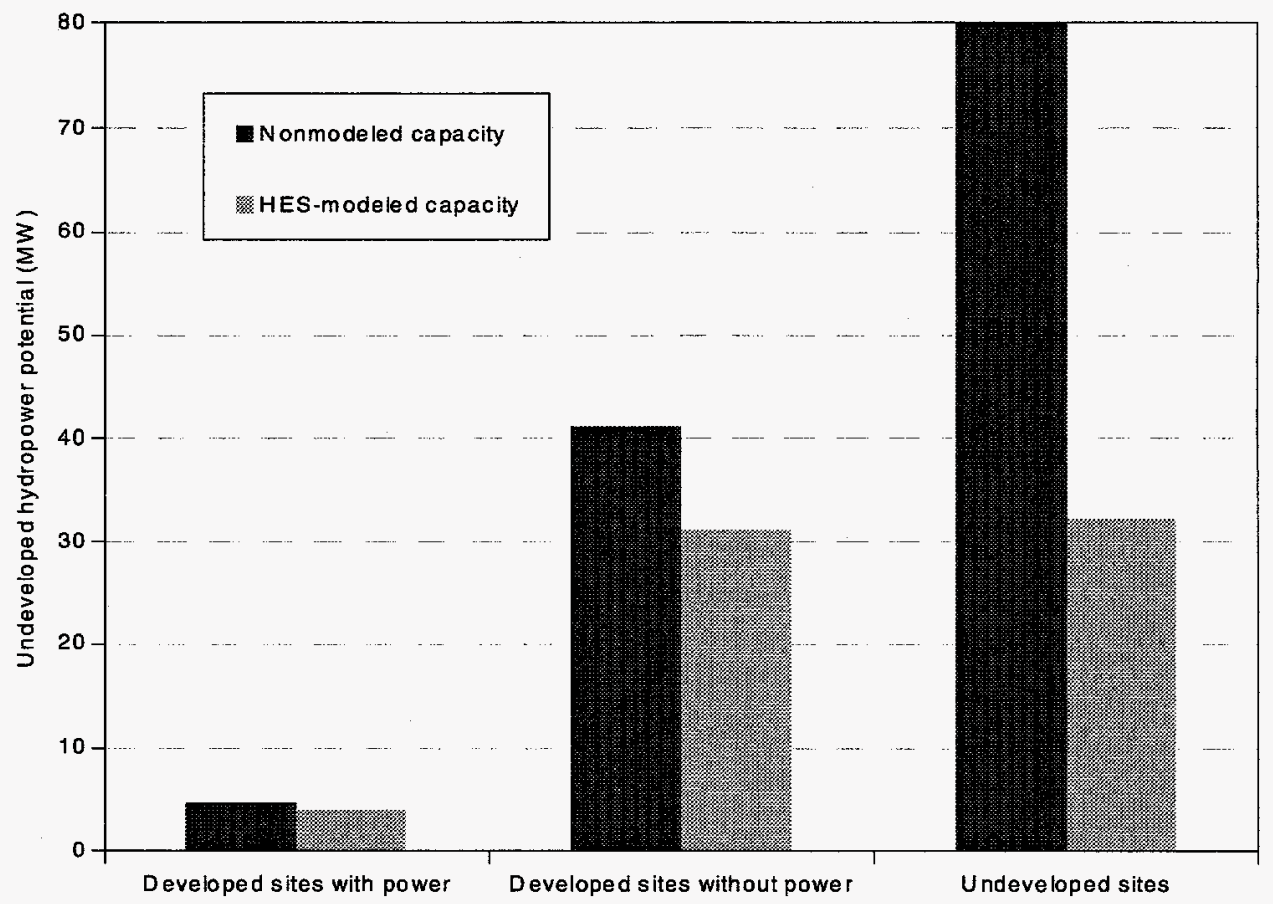

Figure 2. The nonmodeled and HES-modeled undeveloped hydropower potential. 


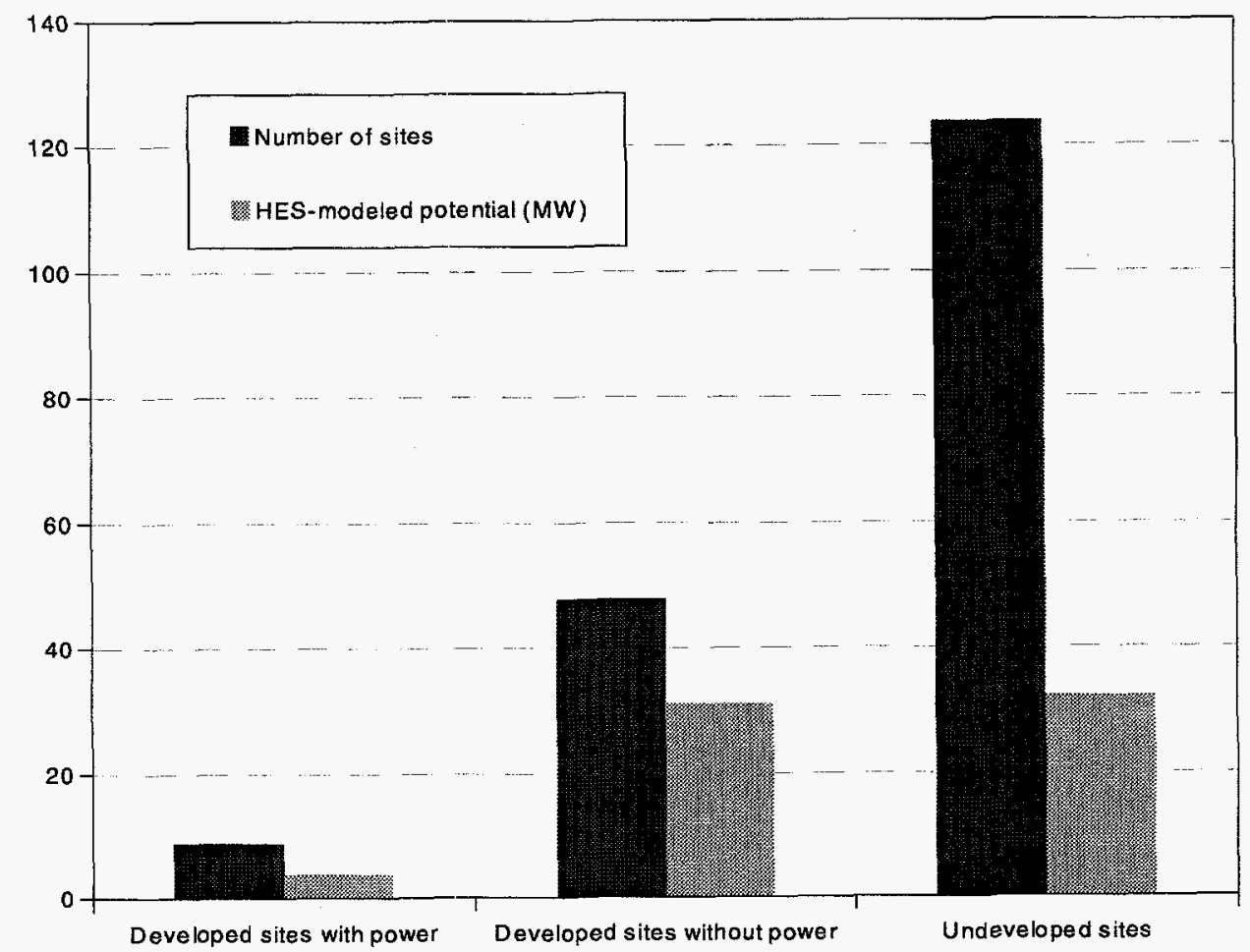

Figure 3. The number of sites with undeveloped hydropower potential and the total megawatts of HES-modeled undeveloped hydropower potential.

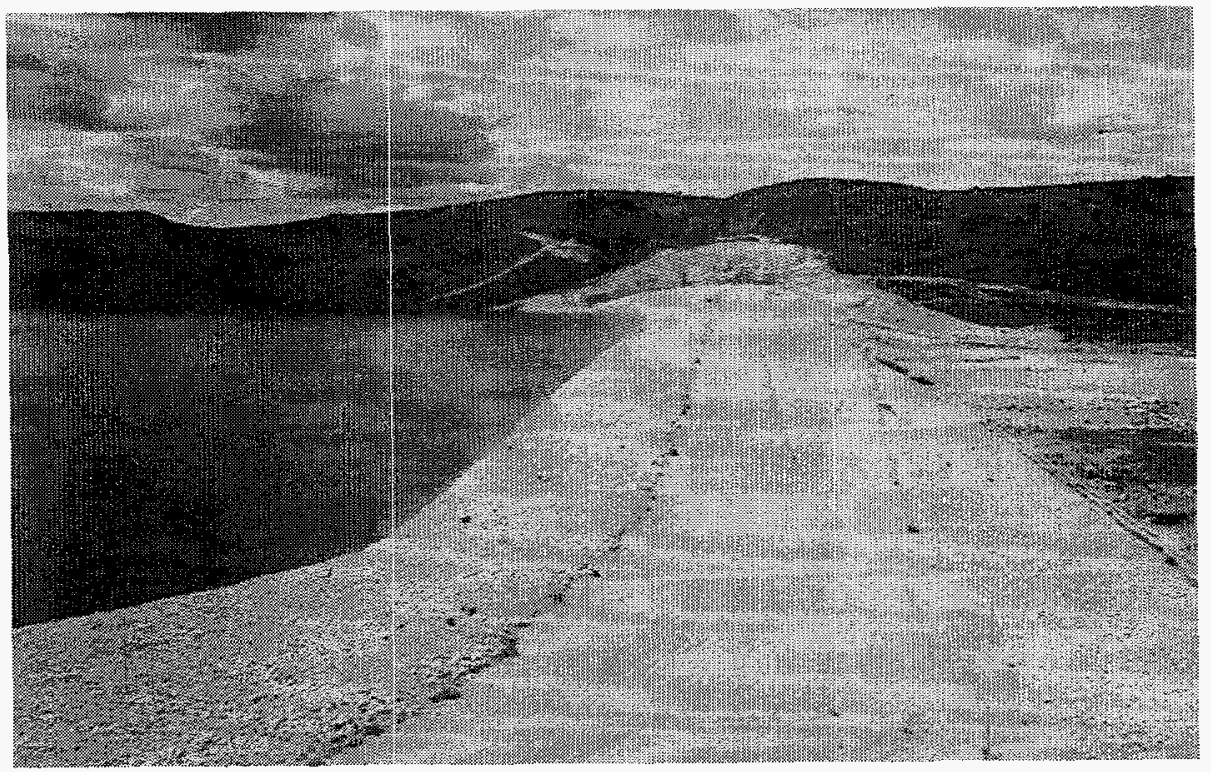

Figure 4. The South Fork Dam, on the South Fork Humbolt River, is an example of an earthfilled embankment structure, 55 feet high, without power generation. 


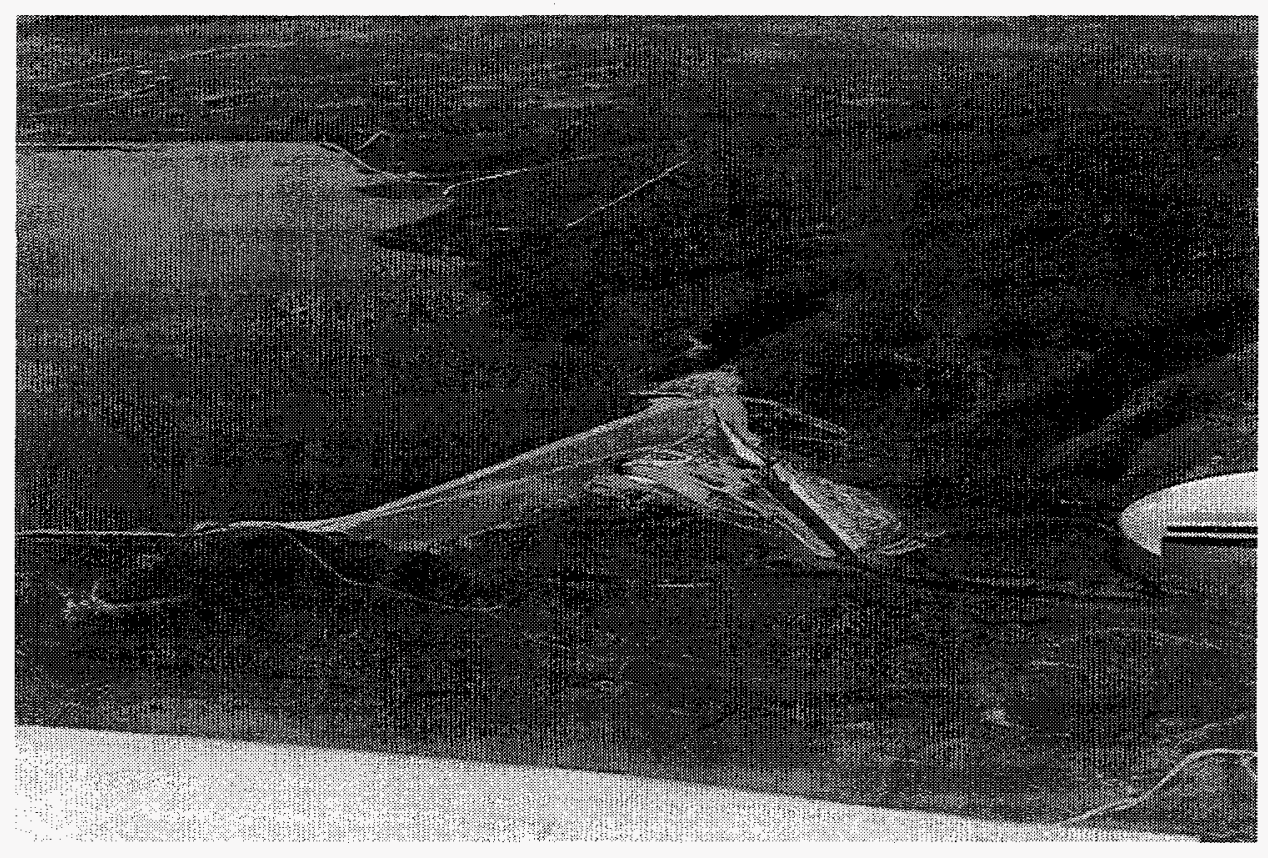

Figure 5. An aerial view of the South Fork Dam, owned by the State of Nevada, Department of Conservation and Natural Resources.

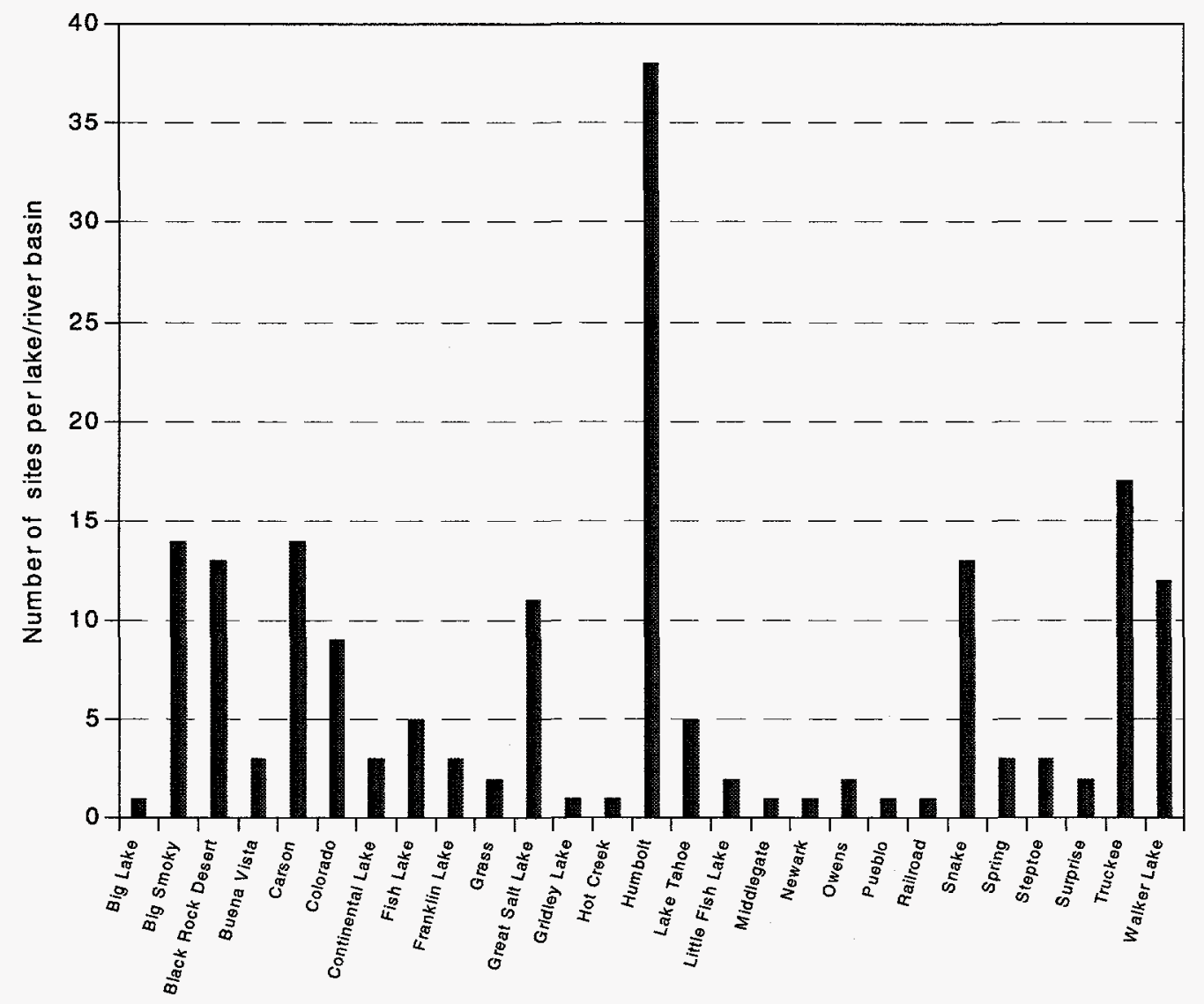

Figure 6. Number of sites with undeveloped hydropower potential in the Nevada river basins. 


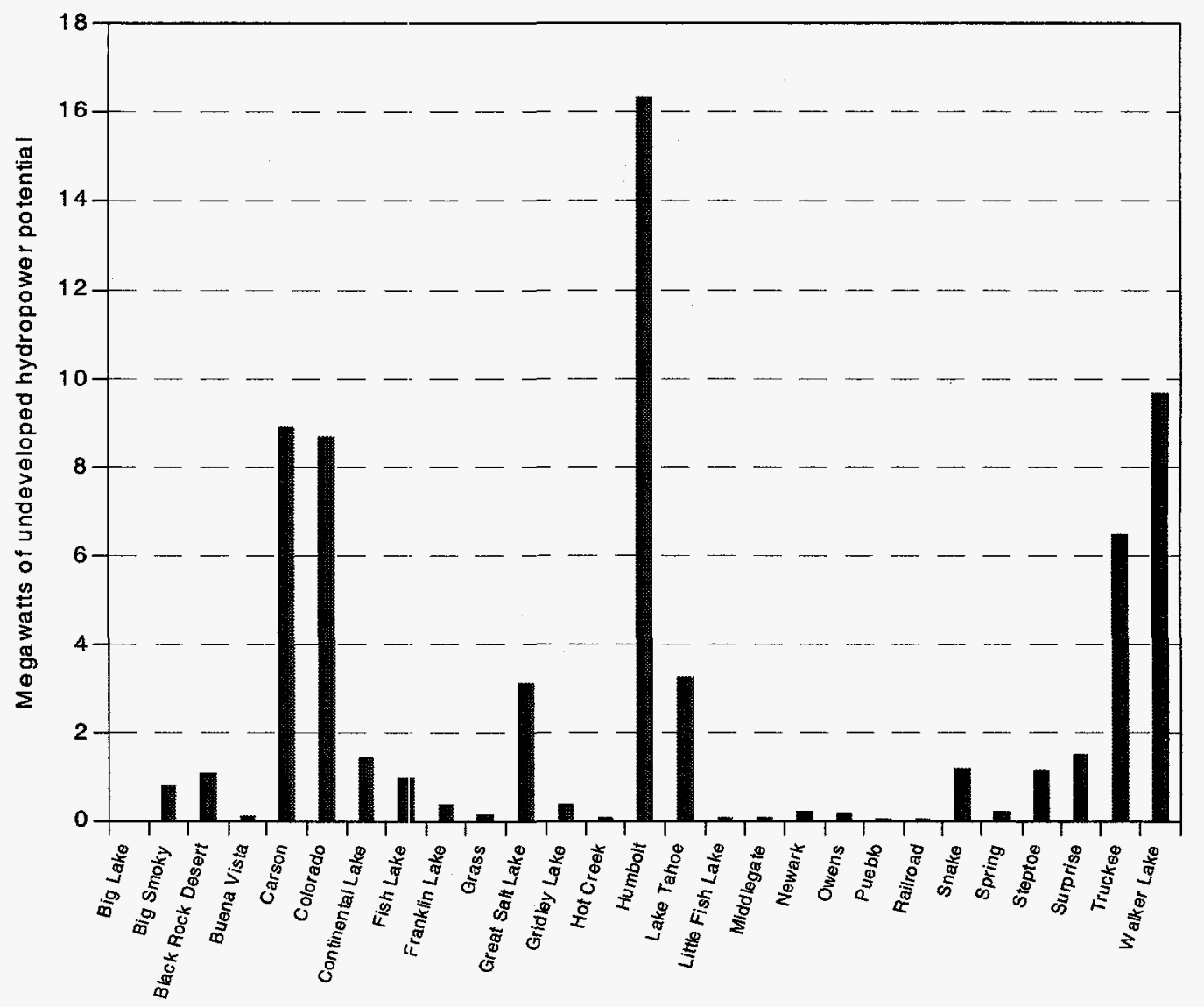

Figure 7. Megawatts of HES-modeled undeveloped hydropower potential in the Nevada river basins.

\section{Detailed Results}

The appendices contain, in the form of HES-generated reports, detailed information about the undeveloped hydropower potential in Nevada. The appendices contain the following information:

Appendix A summarizes the undeveloped hydropower potential by dam status groups. The number of sites, nonmodeled undeveloped hydropower potential, and HES-modeled undeveloped hydropower potential is provicled based on the dam status.

Appendix B provides the hydropower resource assessment by river basin, which includes the project number, project name, stream name, dam status, nonmodeled undeveloped hydropower potential, and the HES-modeled undeveloped hydropower potential for each site. Subtotals are provided for each river basin.
Appendix $\mathbf{C}$ lists the project numbers, plant name, stream name, if a site is Federally owned, nonmodeled undeveloped hydropower potential, and HES-modeled undeveloped hydropower potential. The sites are grouped by dam status.

Appendix D contains a resource database list for the 181 sites in Nevada. Information includes plant name, stream, state, county, river basin and owner names, project number, nameplate and HES-modeled undeveloped hydropower potential, the unit and plant types, dam status, latitude, longitude, and the environmental factors that the HES uses to determine the PESF.

Appendix E lists the additional 201 hydropower sites reported by the State of Nevada. The list is sorted by stream name. 


\section{OBTAINING INDIVIDUAL STATE INFORMATION}

Additional copies of the hydropower resource assessment results for individual states are available and can be obtained by writing or calling the authors or the National Technical Information Service (NTIS).

Telephone Orders-(703) 487-4650. NTIS sales desk and customer services are available between 8:30 a.m. and 5:00 p.m., Eastern Standard Time.

Fax-(703) 321-8547. Customers may fax their orders to NTIS. These orders may be charged to a NTIS deposit account, American Express, VISA, or MasterCard.

Mail Orders-Mail orders should be sent to National Technical Information Service, Document Sales, 5285 Port Royal Road, Springfield, VA 22161. Call the sales desk for prices before placing an order.

E-mail-Customers may e-mail their requests to info@ntis.fedworld.gov.

Method of Payment-Customers may pay for reports (and other NTIS products and services) by (a) credit card (American Express, Visa or MasterCard); (b) check or money order on a United States bank payable to NTIS; (c) NTIS deposit account; or, (d) by asking to be billed (add \$7.50 per order), United States, Canada, and Mexico, only.

Handling Fee-A $\$ 4.00$ handling fee per total order applies to orders from the United States, Canada, and Mexico. Handling charges do not apply to rush order service or pick-up orders.

Postage and Shipping-Orders are shipped first class mail, or equivalent, to addresses in the United States, Canada, and Mexico.

Order Turnaround Time-Orders for technical reports generally are shipped within 3 to 5 days of receipt. For faster service, NTIS offers rush order service.
Rush Order Service-Call 1-800-533-NTIS. In Virginia, Canada, and Mexico call (703) 487-4700. For NTIS rush order service add $\$ 15$ per item. This guarantees that an order will be processed through NTIS within 24 hours of its receipt. These orders receive immediate, individual attention. The items ordered are delivered by first call mail. Call NTIS for information on rush order service for computer products.

For Help in Tracing an Order-Call (703) $487-4650$ and request the customer service option.

\section{ADDITIONAL HYDROPOWER EVALUATION SOFTWARE INFORMATION}

Additional information concerning the HES can be obtained by contacting Ben Rinehart or Jim Francfort at the addresses provided below. Copies of the software and the User's Manual may also be obtained from them.

Ben Rinehart, Project Manager

Idaho National Engineering and Environmental Laboratory

P.O. Box 1625, MS 3830

Idaho Falls, ID 83415-3830

(208) 526-1002

Jim Francfort

Idaho National Engineering and Environmental Laboratory

P.O. Box 1625, MS 3830

Idaho Falls, ID 83415-3830

(208) 526-6787

Information concerning the State of $\mathrm{Ne}$ vada's involvement with the resource assessment or about the identified sites may be obtained by contacting:

Michael J. Anderson, P.E.

State of Nevada, Department of Conservation and Natural Resources

Division of Water Resources

123 W. Nye Lane, Suite 246

Carson City, Nevada 89706-0818

(702) 687-4380 


\section{REFERENCES}

Conner, A. M., J. E. Francfort, and B. N. Rinehart, 1996, Uniform Criteria for U.S. Hydropower Resource Assessment, Hydropower Evaluation Software Status Report-II, DOE/ID 10430.1, Idaho National Engineering Laboratory, Idaho Falls, Idaho.

Francfort, J. E., S. D. Matthews, and B. N. Rinehart, 1991, Hydropower Evaluation Software User's Manual, DOE/DD-10338, Idaho National Engineering Laboratory, Idaho Falls, Idaho. 


\section{Appendix A}

Summary Report 
H Y D R O P O W E R

Number of

state

Category

Projects

NV

with Power

W/O Power

Undeveloped

STATE TOTAL

TOTALS

With Power

W/O Power

Undeveloped

GRAND TOTAL
C A P A C I T Y

Name Plate

Capacity (KW)

4738

41165

80005

125908

4738

41165

80005

125908
DATE: $08 / 19 / 97$

$S$ U M M A R Y

HES Adjusted Capacity (KW)

3915.45

31114.25

32196.25

67225.95

3915.45

31114.25

32196.25

67225.95 
Appendix B

River Basins Report 


\begin{tabular}{|c|c|c|c|c|c|}
\hline & HYDROPOWER RESOURCE & ASSESSMENT BY & RIVER & BASIN & \\
\hline $\begin{array}{l}\text { FERC } \\
\text { Number }\end{array}$ & $\begin{array}{c}\text { Plant Name/ } \\
\text { Stream }\end{array}$ & $\begin{array}{l}\text { Dam Name } \\
\text { stat Rating }\end{array}$ & $\begin{array}{l}\text { Plate } \\
g(\mathrm{KW})\end{array}$ & PESF & $\mathrm{PESF} * \mathrm{KW}$ \\
\hline $\begin{array}{l}\text { ** Rivel } \\
\text { NV00016 }\end{array}$ & $\begin{array}{l}\text { Basin BIG LAKE BASIN } \\
\text { LITTLE VALLEY RESERVOIR } \\
\text { COLEMAN CANYON CREEK }\end{array}$ & wo & 29.00 & 0.90 & 26.10 \\
\hline ** Subtc & otal ** & & 29.00 & & 26.10 \\
\hline $\begin{array}{l}\text { ** River } \\
\text { NV00536 }\end{array}$ & $\begin{array}{l}r \text { Basin BIG SMOKY BASIN } \\
\text { ROUND MOUNTAIN HYDRAULIC } \\
\text { JEFFERSON \& SHOSHONE CREEKS }\end{array}$ & $\mathrm{U}$ & 82.00 & 0.90 & 73.80 \\
\hline NVHO0 052 & $\begin{array}{l}\text { ARC DOME \#1 } \\
\text { SOUTH TWIN RIVER }\end{array}$ & $\mathrm{U}$ & 162.00 & 0.50 & 81.00 \\
\hline NVHO102 & $\begin{array}{l}\text { SPENCER PLANT } \\
\text { BIRCH CREEK }\end{array}$ & $\mathrm{U}$ & 94.00 & 0.50 & 47.00 \\
\hline NVH0103 & $\begin{array}{l}\text { MILLET PLANT } \\
\text { BOWMAN CREEK }\end{array}$ & $\mathbf{U}$ & 45.00 & 0.50 & 22.50 \\
\hline NVH0115 & $\begin{array}{ll}\text { PABLO CANYON PLANT } \\
\text { PABLO CANYON CREEK }\end{array}$ & $\mathrm{U}$ & 95.00 & 0.50 & 47.50 \\
\hline NVH0116 & $\begin{array}{l}\text { BARKER PLANT } \\
\text { BARKER CREEK }\end{array}$ & $\mathrm{U}$ & 69.00 & 0.90 & 62.10 \\
\hline NVHO117 & $\begin{array}{l}\text { NOT P.C. PLANT } \\
\text { BROAD CREEK }\end{array}$ & $\mathrm{U}$ & 104.00 & 0.50 & 52.00 \\
\hline NVH0118 & $\begin{array}{l}\text { COVE PLANT } \\
\text { COVE CANYON CREEK }\end{array}$ & $\mathrm{U}$ & 67.00 & 0.50 & 33.50 \\
\hline NVH0119 & $\begin{array}{l}\text { ARC DOME \#2 } \\
\text { NORTH TWIN RIVER }\end{array}$ & $\mathrm{U}$ & 134.00 & 0.50 & 67.00 \\
\hline NVHO 120 & $\begin{array}{l}\text { LAST CHANCE PLANT } \\
\text { LAST CHANCE CREEK }\end{array}$ & $\mathrm{U}$ & 78.00 & 0.50 & 39.00 \\
\hline NVHO121 & $\begin{array}{l}\text { GOLDRUSH PLANT } \\
\text { OPHIR CREEK }\end{array}$ & $\mathrm{U}$ & 78.00 & 0.50 & 39.00 \\
\hline NV00127 & $\begin{array}{l}\text { GROVES LAKE } \\
\text { KINGSTON CREEK }\end{array}$ & Wo & 225.00 & 0.75 & 168.75 \\
\hline NV00191 & $\begin{array}{l}\text { SEYLER RESERVOIR } \\
\text { PEAVINE CREEK }\end{array}$ & Wo & 53.00 & 0.90 & 47.70 \\
\hline NV03898 & $\begin{array}{l}\text { ROUND MOUNTAIN } \\
\text { JETT CREEK }\end{array}$ & wo & 82.00 & 0.90 & 73.80 \\
\hline
\end{tabular}


Page No.

$08 / 19 / 97$

FERC

Number
Plant Name/

stream
Dam Name Plate

stat Rating (KW) PESF PESF * KW

** Subtotal **

1368.00

854.65

** River Basin BLACK ROCK DESERT BASIN

NVHO035 LEONARD RANCH PLANT
LEONARD CREEK

$\mathrm{U}$

$\mathrm{U}$

KING'S RIVER

NVH0037 LOG CABIN PLANT

LOG CABIN CREEK

NVH0038 GRANITE PLANT

GRANITE CREEK

NVH0039 MCDERMITT \#1

EAST QUINN RIVER

NVH0040 MCDERMITT \#2

MCDERMITT CREEK

NVH0089 UPPER SOLDIER MEADOWS COLEMAN CREEK

NVHO092 SUMMIT PLANT

SUMMER CAMP CREEK

NVH0093 BATTLE CREEK PLANT

BATTLE CREEK

NVH0095 WILLOW CREEK PLANT BIG CREEK

NVH0096 REBEL YELL PLANT REBEL CREEK

NV00024 BILK CREEK RESERVOIR BILK CREEK

NV00197 SOLDIER MEADOWS RESERVOIR SOLDIER CREEK

$\mathrm{U}$

U

$\mathrm{U}$

U

U

$\mathrm{U}$

$\mathrm{U}$

$\mathrm{U}$
$96.00 \quad 0.90 \quad 86.40$

$54.00 \quad 0.90$

48.60

$43.00 \quad 0.90$

38.70

$29.00 \quad 0.90$

26.10

$136.00 \quad 0.50$

68.00

55.80

$62.00 \quad 0.90$

$69.00 \quad 0.90$

62.10

60.30

$67.00 \quad 0.90$

$262.00 \quad 0.90$

235.80

$130.00 \quad 0.90$

117.00

$67.00 \quad 0.50$

33.50

wo

$185.00 \quad 0.90$

166.50

wo

$116.00 \quad 0.90$

104.40

** Subtotal ** 
Page No.

$08 / 19 / 97$

$\begin{array}{cc}\text { FERC } & \text { Plant Name/ } \\ \text { Number } & \text { Stream }\end{array}$

** River Basin BUENA VISTA BASIN

NVH0126 UNIONVILLE \#2 INDIAN CREEK

NVH0127 UNIONVILLE \#3 COTTONWOOD CREEK

NVH0128 UNIONVILIE \#1 BUENA VISTA CREEK

** Subtotal **

** River Basin CARSON RIVER BASIN

06133 BROKEN DAM

EAST FORK CARSON RIVER

NV001 WATASHEAMU

EAST FORK CARSON RIVER

NV20004 REDHEAD DAM

EAST FORK CARSON RIVER

NV2U003 MEXICAN DAM

CARSON RIVER

NVHO004 CLEAR CREEK

CLEAR CREEK

NVH0006 BRYANT CREEK

BRYANT CREEK

NVHO068 MOTT CANYON PLANT

MOTT CREEK

NVH0069 MOUNT SEIGEL PLANT

PINE NUT CREEK

NVH0125 CARSON CITY

ASH CANYON CREEK

NVU0018 EUREKA DAM

CARSON RIVER

NV80000 26 FOOT DROP POWER PLANT $\mathrm{V}$ CANAL

NVO0234 EAST PEAK LAKE

DAGGETT CREEK
Dam Name Plate

stat Rating (KW) PESF PESF * KW

U

$43.00 \quad 0.90$

38.70

U

$43.00 \quad 0.90$

38.70

U

$79.00 \quad 0.90$

71.10

165.00

148.50

$\mathrm{U}$

$2000.00 \quad 0.10$

200.00

U

$8000.00 \quad 0.10$

800.00

U

$2700.00 \quad 0.25$

675.00

U

$3500.00 \quad 0.50$

1750.00

$\mathrm{U}$

$198.00 \quad 0.25$

49.50

U

$104.00 \quad 0.50$

52.00

U

$163.00 \quad 0.90$

146.70

$\mathrm{U}$

$839.00 \quad 0.25$

209.75

$\mathrm{U}$

$244.00 \quad 0.90$

219.60

$\mathrm{U}$

$6000.00 \quad 0.25$

1500.00

W

$1600.00 \quad 0.90$

1440.00

พo

$131.00 \quad 0.90$

117.90 
Page No.

$08 / 19 / 97$

$\begin{array}{cc}\text { FERC } & \text { Plant Name/ } \\ \text { Number } & \text { Stream }\end{array}$

NV10120 CARSON RIVER DIVERSION CARSON RIVER

NV10133 STILLWATER POINT CARSON RIVER

** Subtotal **

** River Basin COLORADO RIVER BASIN

05423A LAS VEGAS WASH 2

LAS VEGAS WASH

05423B LAS VEGAS WASH 3

LAS VEGAS WASH

10463 S NEVADA TREATMENT

LAS VEGAS EFFLUENT

10577 WETLANDS RECOVEROS CONTRI LAS VEGAS EFFLUENT

NVHOOO2 OVERTON PLANT

VIRGIN RIVER

NVHO048 URSINE PLANT

MEADOW VALLEY WASH

NVH0144 PRIOR TO PRESTON PLANT'

WHITE RIVER

10958 LAS VEGAS WASH

LAS VEGAS WASH

NV00113 BOWMAN RESERVOIR MUDDY RIVER

** Subtotal **
Wo

wo

Dam

stat Rating (KW) PESF

$2200.00 \quad 0.75$

$1100.00 \quad 0.10$

28779.00

** River Basin CONTINEN
05602B ALDER CREEK 2

ALDER CREEK

05602A ALDER CREEK 1 ALDER CREEK
U

$\mathrm{U}$

$\mathrm{U}$

U

$\mathrm{U}$

U

$\mathrm{U}$

Wo

Wo

14518.00

U

$$
400.00 \quad 0.90 \quad 360.00
$$$$
300.00 \quad 0.90
$$$$
270.00
$$$$
6000.00 \quad 0.50 \quad 3000.00
$$$$
3150.00 \quad 0.50
$$$$
1575.00
$$

215.00

29.25

951.30

2085.00

255.60

$284.00 \quad 0.90$

8741.15

$275.00 \quad 0.90$

247.50

wo

$1300.00 \quad 0.90$

1170.00 
Page No.

$08 / 19 / 97$

FERC

Number

NV00005 ONION RESERVOIR

LITTLE ALDER CREEK

** Subtotal **

** River Basin FISH LAKE BASIN

NVH0031 MOUNT BARCROFT \#1

PERRY-AIKEN CREEK

NVH0033 INDIAN CREEK PLANT

INDIAN CREEK

$\begin{array}{ll}01746 & \text { LEIDY } \\ & \text { LEIDY (CIRCLE L) }\end{array}$

NV10222 B-B MINE RESERVOIR ROCK CREEK

NV10223 CHIATOVICH CREEK RESERVOIR CHIATOVICH CREEK

** Subtotal **

* * River Basin FRANKLIN LAKE BASIN

NVH0028 OVERLAND PLANT

NVH0029 FRANKLIN LAKE PLANT

FRANKLIN RIVER

NVH0081 FRANKLIN \#3

MEYER'S CREEK

** Subtotal **

** River Basin GRASS BASIN

NVH0105 CALLAGHAN \#2

STEINER CREEK

NV10460 CALLAGHAN CREEK RESERVOIR CALLAGHAN CREEK
OVERLAND CREEK

Dam Name Plate

stat Rating (KW) PESF PESF * KW

wo

$76.00 \quad 0.75$

57.00

1651.00

1474.50

$\mathrm{U}$

$313.00 \quad 0.90$

281.70

$\mathrm{U}$

$167.00 \quad 0.90$

150.30

W

$200.00 \quad 0.75$

150.00

wo

$285.00 \quad 0.90$

256.50

wo

$177.00 \quad 0.90$

159.30

1142.00

997.80

$\mathrm{U}$

$323.00 \quad 0.90$

290.70

U

$230.00 \quad 0.50$

115.00

$\mathrm{U}$

$$
43.00 \quad 0.50
$$

21.50

596.00

427.20

$\mathrm{U}$

$110.00 \quad 0.90$

99.00

wo

$83.00 \quad 0.90$

74.70

** Subtotal ** 
Page No.

$08 / 19 / 97$

$\begin{array}{cc}\text { FERC } & \text { Plant Name/ } \\ \text { Number } & \text { Stream }\end{array}$

** River Basin GREAT SALT LAKE BASIN

NV45518 LEHMAN PLANT

LEHMAN CREEK \& TRIBUTARIES

NV49555 RED POINT PLANT

THOUSAND SPRINGS CREEK

NV49556 ECCLES PLANT

THOUSAND SPRINGS CREEKK

NVH0063 GOSHUTE PLANT

SPRING CREEK

NVH0065 BAKER PLANT

BAKER CREEK

NVH0139 KALAMAZOO PLANT
KALAMAZOO CREEK

NVH0142 BIG WASH PLANT

BIG WASH CREEK

NVHO143 SNAKE PLANT

SNAKE CREEK

NV00109 DAEK RESERVOIR

THOUSAND SPRINGS CREEK

NV00110 TWENTY THREE MILE RES. THOUSAND SPRINGS CREEK

NV00112 SILVER CREEK DAM SILVER CREEK

** Subtotal **

** River Basin GRIDLEY LAKE BASIN

05619 KNOTT CREEK

KNOTT CREEK

** Subtotal **

** River Basin HOT CREEK BASIN

NVHO124 SOUTH SIXMILE CANYON

SIXMILE CREEK
U

$128.00 \quad 0.90$

115.20

U

$76.00 \quad 0.90$

68.40

$\mathrm{U}$

$76.00 \quad 0.90$

68.40

$\mathrm{U}$

$64.00 \quad 0.50$

32.00

U

$161.00 \quad 0.90$

144.90

U

$211.00 \quad 0.50$

105.50

U

$185.00 \quad 0.50$

92.50

U

$165.00 \quad 0.50$

82.50

Wo

$1300.00 \quad 0.90$

1170.00

wo

$1100.00 \quad 0.90$

990.00

wo

$311.00 \quad 0.90$

279.90

3777.00

3149.30

wo

$554.00 \quad 0.75$

415.50

554.00

415.50

U

$106.00 \quad 0.90$

95.40 


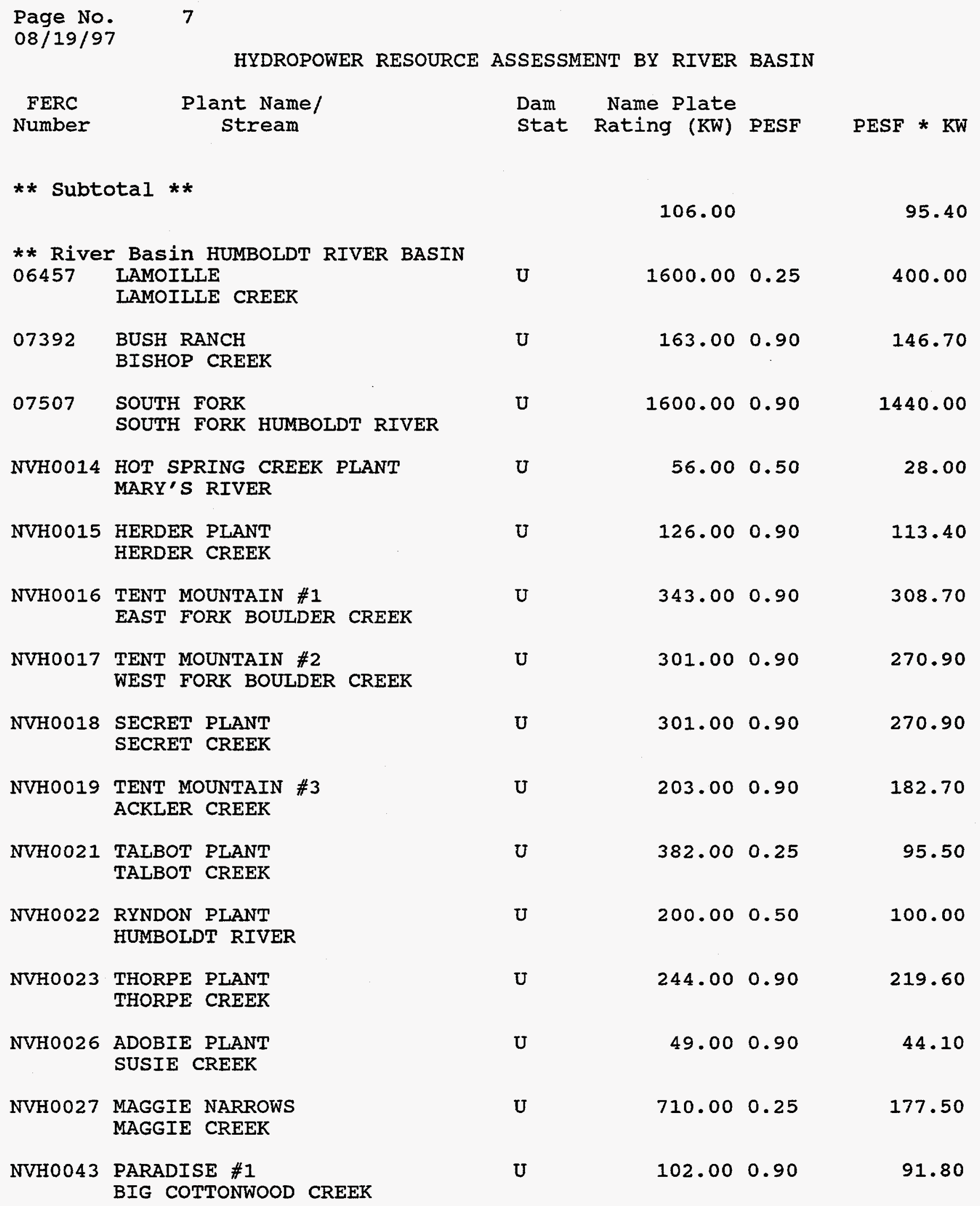


Page No.

$08 / 19 / 97$

$\begin{array}{cc}\text { FERC } & \text { Plant Name/ } \\ \text { Number } & \text { Stream }\end{array}$

NVH0044 PARADISE \#2

INDIAN CREEK

NVH0045 GOLCONDA PLANT

POLE CREEK

NVH0046 ROCK CREEK DAM

ROCK CREEK

NVHO051 YOMBA PLANT

REESE RIVER

NVH0080 DIXIE FLATS PLANT

DIXIE CREEK

NVH0097 COLONY CREEK PLANT

SINGAS CREEK

NVH0098 PARADISE \#2

WASH O'NEIL CREEK

NVHO099 STONE HOUSE PLANT

ABEL CREEK

NVH0100 COIEMAN CREEK PLANT

MULLINEX CREEK

NVH0101 AUSTIN-50 PLANT

BIG CREEK

NVHO112 CORRAL WASH PLANT

CLEAR CREEK

NVU0017 DEVIL'S GATE RESERVOIR

NORTH FORK HUMBOLDT RIVER

NVHO042 PARADISE MILL

MARTIN CREEK

08392 RYE PATCH

HUMBOLDT RIVER

11311A TS RANCH HYDRO 1

GOLD MINE DISCHARGE PIPELINE

11311B TS RANCH HYDRO 2

GOLD MINE DISCHARGE PIPELINE
Dam Name Plate
stat Rating (KW) PESF PESF * KW

U

$183.00 \quad 0.50$

91.50

$\mathrm{U}$

$73.00 \quad 0.90$

65.70

$\mathrm{U}$

$137.00 \quad 0.25$

34.25

$\mathrm{U}$

$89.00 \quad 0.50$

44.50

$\mathrm{U}$

$99.00 \quad 0.90$

89.10

$\mathrm{U}$

$52.00 \quad 0.90$

46.80

U

$53.00 \quad 0.90$

47.70

U

$49.00 \quad 0.90$

44.10

U

$106.00 \quad 0.90$

95.40

U

$63.00 \quad 0.50$

31.50

U

$41.00 \quad 0.50$

20.50

U

$1900.00 \quad 0.90$

1710.00

W

$126.00 \quad 0.75$

94.50

wo

$1400.00 \quad 0.75$

1050.00

wo

$2500.00 \quad 0.75$

1875.00

wo

$2000.00 \quad 0.75$

1500.00 
Page No.

$08 / 19 / 97$

$\begin{array}{cc}\text { FERC } & \text { Plant Name/ } \\ \text { Number } & \text { Stream }\end{array}$

NV00050 METROPOLIS RESERVOIR

BISHOP CREEK

NV00051 BOYD RESERVOIR

RABBIT CREEK

NV00055 ZUNINO RESERVOIR

SMITH CREEK

NV00063 LOWER PITT-TAYLOR RES.

HUMBOLDT RIVER

NV00226 SOUTH FORK RESERVOIR

SOUTH FORK HUMBOLDT RIVER

NV01151 CHIMNEY RESERVOIR

LITTLE HUMBOLDT RIVER

NV10114 PALISADES \#2

PINE CREEK

** Subtotal **

** River Basin LAKE TAHOE BASIN

NVH0133 DIAMOND PEAK PLANT

INCLINE CREEK

08703 NEVADA STATE SUPPLY NV ST CONDUIT (MARLETTE LK)

09530 TAHOE TUNNEL

IAKE TAHOE EFFLUENT PIPELINE

NV00199 INCLINE LAKE

THIRD CREEK

NV10170 LOGAN CREEK

LOGAN CREEK

** Subtotal **

** River Basin LITTLE FISH LAKE BASIN

NVH0122 TEXTILE PLANT

DANVILLE CREEK

NVH0123 WINDOW PLANT

CLEAR CREEK
Dam Name Plate
Stat Rating (KW) PESF PESF * KW

wo

$1800.00 \quad 0.90$

1620.00

Wo

$250.00 \quad 0.90$

225.00

WO

$107.00 \quad 0.75$

80.25

Wo

$1500.00 \quad 0.90$

1350.00

Wo

$2900.00 \quad 0.75$

2175.00

wo

$95.00 \quad 0.75$

71.25

wo

$$
77.00 \quad 0.90
$$

69.30

21980.00

16321.15

U

$110.00 \quad 0.90$

99.00

wo

$1100.00 \quad 0.75$

825.00

wo

$2500.00 \quad 0.75$

1875.00

wo

$422.00 \quad 0.75$

316.50

Wo

$203.00 \quad 0.75$

152.25

4335.00

3267.75

U

$96.00 \quad 0.50$

48.00

U

$82.00 \quad 0.50$

41.00 
Page No.

$08 / 19 / 97$

FERC

Number

HYDROPOWER RIESOURCE ASSESSMENT BY RIVER BASIN

Plant Name/

stream
Dam Name Plate

stat Rating (KW) PESF PESF * KW

** Subtotal **

178.00

89.00

** River Basin MIDDLEGATE BAS:CN

NVHO129 BASQUE PLANT

EDWARDS CREEK

$\mathrm{U}$

$94.00 \quad 0.90$

84.60

** Subtotal **

$$
94.00
$$

84.60

** River Basin NEWARK BASIN

09618 ROBINSON SPRINGS

ROBINSON SPRINGS

wo

$300.00 \quad 0.75$

225.00

** Subtotal **

300.00

225.00

** River Basin OWENS RIVER BASIN

NV10322 RATTLESNAKE RESERVOIR

QUEEN CREEK

NVHO083 QUEEN RANCH PLANT

MORRIS CREEK

WO

$140.00 \quad 0.90$

126.00

wo

$142.00 \quad 0.50$

71.00

** Subtotal **

282.00

197.00

** River Basin PUEBLO BASIN

NVH0085 TRIDENT \#2

WILDER CREEK

U

$75.00 \quad 0.90$

67.50

** Subtotal **

75.00

67.50

** River Basin RAILROAD BASIN

NVH0053 CURRANT PLANT

CURRANT CREEK

$\mathrm{U}$

$81.00 \quad 0.90$

72.90

** Subtotal **

81.00

72.90

** River Basin SNAKE RIVER BASIN

NVHOOO9 CHICKEN CREEK SUMMIT

JACK CREEK

U

$120.00 \quad 0.25$

30.00

NVHO011 ROWLAND PLANT

BRUNEAU RIVER

$\mathrm{U}$

$617.00 \quad 0.10$

61.70 
Page No.

$08 / 19 / 97$

FERC

Number

HYDROPOWER RESOURCE ASSESSMENT BY RIVER BASIN

Plant Name/

Stream
Dam Name Plate
Stat Rating (KW) PESF PESF * KW

$\mathrm{U}$

$102.00 \quad 0.25$

25.50

COON CREEK

$\mathrm{U}$

$58.00 \quad 0.50$

29.00

COPPER CREEK

NVH0073 BIG BUCK PLANT

BUCK CREEK

NVH0074 DAVE POWER PLANT

DAVE CREEK

NVH0075 JARBIDGE \#1

EAST FORK JARBIDGE RIVER

NVH0076 CANYON PLANT

CANYON CREEK

NVU0001 PATSVILLE OWYHEE RIVER

NVU0002 SKULL CREEK

OWYHEE RIVER

NV03720 CURLEY-WOOD

JARBIDGE RIVER

U

$197.00 \quad 0.10$

19.70

U

$91.00 \quad 0.10$

9.10

U

$260.00 \quad 0.10$

26.00

U

$102.00 \quad 0.10$

10.20

$\mathrm{U}$

1600.000 .10

160.00

$\mathrm{U}$

$3400.00 \quad 0.10$

340.00

W

พอ

wo

NV10189 HEADGATE DAM

SALMON FALLS CREEK

** Subtotal **

** River Basin SPRING BASIN

NV07847 PIERMONT MILL

PIERMONT CREEK

NV45749 VIPONT CREEK PLANT STEPHENS CREEK

NV45750 CLEVELAND RANCH PLANT CLEVE CREER
7282.00

$\mathrm{U}$

1210.20

76.50

U

U

$85.00 \quad 0.90$

$65.00 \quad 0.90$

58.50

103.50
152.50

144.00

202.50

$270.00 \quad 0.75$ 
Page No.

$08 / 19 / 97$

\section{FERC}

Number

$$
\text { Plant Name/ }
$$

stream

** Subtotal **

** River Basin STEPTOE BASIN

NVH0138 BIG INDIAN PLANT

BIG INDIAN CREEK

NVU0023 KEYSTONE RESERVOIR

GLEASON CREEK

NV00174 COMINS LAKE

STEPTOE CREEK

** Subtotal **

** River Basin SUPRISE BASIN

NV00022 WALL CANYON \#1

WALL CREEK

NV00023 WALL CANYON \#2

WALL CREEK

** Subtotal **

** River Basin TRUCKEE RIVER BASIN

07779 GALENA CREEK

GALENA CREEK

08973 TRUCKEE

TRUCKEE RIVER

09646 HUNTER CREEK

HUNTER CREEK

NV2U001 LAWTON DAM

TRUCKEE RIVER

NV2U002 VERDI DAM

TRUCKEE RIVER

NV2U005 PEKOE DAM

TRUCKEE RIVER

NVH0055 WHITE'S PLANT

WHITE'S CREEK
Dam Name Plate
Stat Rating (KW) PESF PESF * KW

$$
265.00
$$

238.50

U

$79.00 \quad 0.50$

39.50

U

$1200.00 \quad 0.90$

1080.00

wo

$102.00 \quad 0.75$

76.50

1381.00

1196.00

wo

$1100.00 \quad 0.90$

990.00

wo

$$
600.00 \quad 0.90
$$

540.00

1700.00

1530.00

$\mathrm{U}$

$300.00 \quad 0.50$

150.00

$\mathrm{U}$

$1500.00 \quad 0.50$

750.00

$\mathrm{U}$

$270.00 \quad 0.90$

243.00

$\mathrm{U}$

$4100.00 \quad 0.10$

410.00

U

$4000.00 \quad 0.10$

400.00

$\mathrm{U}$

$2000.00 \quad 0.10$

200.00

U

$213.00 \quad 0.90$

191.70 
Page No.

$08 / 19 / 97$

FERC

Number

Plant Name/

stream

NVH0134 GREY POWER PLANT

WEST FORK GREY CREEK

NVH0136 MOUNT ROSE PLANT WEST

DEEP CANYON

NVHO137 UNNAMED PLANT

(NO NAME)

04164

WASHOE

TRUCKEE R

NV01412 OPHIR POWER PLANT

OPHIR CREEK

NV06850 BOWERS MANSION PLANT

FRANKTOWN CREEK

NV09344 PECETTI

THOMAS CREEK

NV01198 FLORISTON MILL

BRONCO AND DRY PRARIE CREEKS

NV10121 DERBY DIVERSION DAM

TRUCKEE RIVER

NV10472 WASHOE LAKE

STEAMBOAT CREEK

** Subtotal **

** River Basin WALKER LAKE BASIN

NV2UC06 HUDSON RESERVOIR

WEST WALKER RIVER

NVHO008 SMITH VALLEY

DESERT CREEK

NVH0049 EAST FORK POWER PLANT

EAST FORK WALKER RIVER

NVH0106 PINE GROVE PLANT

DAIZELL CREEK

NVH0108 BODIE PIANT

BODIE CREEK
Dam Name Plate

stat Rating (KW) PESF PESF * KW

$\mathrm{U}$

$189.00 \quad 0.90$

170.10

$\mathrm{U}$

$427.00 \quad 0.50$

213.50

U

$305.00 \quad 0.90$

274.50

W

$900.00 \quad 0.90$

810.00

W

$335.00 \quad 0.75$

251.25

W

$811.00 \quad 0.90$

729.90

W

$142.00 \quad 0.90$

127.80

wo

$427.00 \quad 0.90$

384.30

wo

wo

$2300.00 \quad 0.50$

1150.00

$69.00 \quad 0.75$

51.75

18288.00

6507.80

U

$1400.00 \quad 0.50$

700.00

U

$427.00 \quad 0.50$

213.50

U

$108.00 \quad 0.50$

54.00

U

$793.00 \quad 0.50$

396.50

U

$854.00 \quad 0.90$

768.60 
Page No.

$08 / 19 / 97$

\section{FERC}

Number

NVH0109 ROUGH WATER PLANT ROUGH CREEK

NVH0110 COTTONWOOD COTTONWOOD CREEK

NVU0020 HOYE CANYON WEST WALKER RIVER $\begin{array}{ll}06420 & \text { SWEETWATER RANCH } \\ \text { SWEETWATER CREEK }\end{array}$

NV10101 CAT CREEK RESERVOIR CAT CREEK

NV10132 WEBER DAM WALKER RIVER

NV10470 TOPAZ RESERVOIR WALKER RIVER
Dam Name Plate
stat Rating (KW) PESF PESF * KW

$U$

$470.00 \quad 0.90$

423.00

$\mathrm{U}$

$1372.00 \quad 0.50$

686.00

$\mathrm{U}$

$3400.00 \quad 0.50$

1700.00

W

$319.00 \quad 0.50$

159.50

wo

$630.00 \quad 0.50$

315.00

wo

$1700.00 \quad 0.75$

พo

$4000.00 \quad 0.75$

15473.00

9691.10

125908.00

67225.95 
Appendix C

Nevada Sites List 
HYDROPOWER RESOURCE ASSESSMENT BY FERC NUMBER

\section{Plant Name/ stream}

** FERC Number 05423A

LAS VEGAS WASH 2

LAS VEGAS WASH

** Subtotal **

** FERC Number 05423B

LAS VEGAS WASH 3

LAS VEGAS WASH

** Subtotal **

** FERC Number 05602B

ALDER CREEK 2

ALDER CREEK

** Subtotal **

** FERC Number 06133

BROKEN DAM

EAST FORK CARSON RIVER

** Subtotal **

** FERC Number 06457

LAMOILLE

LAMOILLE CREEK

** Subtotal **

** FERC Number 07392

BUSH RANCH

BISHOP CREEK

** Subtotal **

** FERC Number 07507

SOUTH FORK

SOUTH FORK HUMBOLDT RIVER

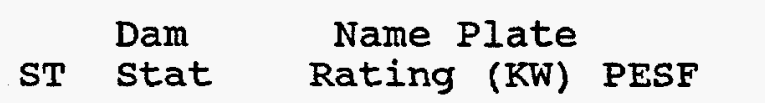

$\mathrm{PESF} * \mathrm{KW}$

NV U

$$
400.00 \quad 0.90
$$

360.00

$$
400.00
$$

360.00

NV U

300.00

0.90

270.00

300.00

270.00

NV U

275.00

0.90

247.50

275.00

247.50

NV U

2000.00

0.10

200.00

2000.00

200.00

NV U

$1600.00 \quad 0.25$

400.00

1600.00

400.00

NV U

$163.00 \quad 0.90$

146.70

163.00

146.70

NV U

$1600.00 \quad 0.90$

1440.00 
Plant Name/

stream

** Subtotal **

** FERC Number 07779

GALENA CREEK

GALENA CREEK

** Subtotal **

** FERC Number 08973

TRUCKEE

TRUCKEE RIVER

** Subtotal **

** FERC Number 09646

HUNTER CREEK

HUNTER CREEK

** Subtotal **

** FERC Number 10463

$S$ NEVADA TREATMENT

IAS VEGAS EFFLUENT

** Subtotal **

** FERC Number 10577

WETIANDS RECOVEROS CONTRI

IAAS VEGAS EFFLUENT

** Subtotal **

** FERC Number NV001

WATASHEAMU

EAST FORK CARSON RIVER

** Subtotal ** $\begin{array}{lll}\text { Dam } & \text { Name Plate } & \\ \text { ST Stat } & \text { Rating (KW) PESF } & \text { PESF * KW }\end{array}$

1600.00

1440.00

NV U

$300.00 \quad 0.50$

150.00

300.00

150.00

NV U

$1500.00 \quad 0.50$

750.00

1500.00

750.00

NV U

$270.00 \quad 0.90$

243.00

270.00

243.00

NV U

$6000.00 \quad 0.50$

3000.00

6000.00

3000.00

NV U

$3150.00 \quad 0.50$

1575.00

3150.00

1575.00

NV U

$8000.00 \quad 0.10$

800.00

8000.00

800.00 
Plant Name/

stream

** FERC Number NV00536

ROUND MOUNTAIN HYDRAULIC

JEFFERSON \& SHOSHONE CREEKS

** Subtotal **

** FERC Number NV07847

PIERMONT MILL

PIERMONT CREEK

** Subtotal **

** FERC Number NV20004

REDHEAD DAM

EAST FORK CARSON RIVER

** Subtotal **

** FERC Number NV2U001

LAWTON DAM

TRUCKEE RIVER

** Subtotal **

** FERC Number NV2U002

VERDI DAM

TRUCKEE RIVER

** Subtotal **

** FERC Number NV2U003

MEXICAN DAM

CARSON RIVER

** Subtotal **

** FERC Number NV2U005

PEKOE DAM

TRUCKEE RIVER

\author{
Dam Name Plate \\ $S T$ stat Rating (KW) PESF PESF * $\mathrm{KW}$
}

NV U

$82.00 \quad 0.90$

73.80

$$
82.00
$$

NV U

$85.00 \quad 0.90$

76.50

85.00

76.50

NV U

$2700.00 \quad 0.25$

675.00

2700.00

675.00

NV U

$4100.00 \quad 0.10$

410.00

4100.00

410.00

NV U

4000.00

0.10

400.00

4000.00

400.00

NV U

$3500.00 \quad 0.50$

1750.00

3500.00

1750.00

NV U

$2000.00 \quad 0.10$

200.00 
Plant Name/ stream

** Subtotal **

** FERC Number NV2UC06

HUDSON RESERVOIR

WEST WALKER RIVER

** Subtotal **

** FERC Number NV45518

LEHMAN PLANT

IEHMAN CREEK \& TRIBUTARIES

** Subtotal **

** FERC Number NV45749

VIPONT CREEK PLANT

STEPHENS CREEK

** Subtotal **

** FERC Number NV45750

CLEVELAND RANCH PLANT

CLEVE CREEK

** Subtotal **

** FERC Number NV49555

RED POINT PLANT

THOUSAND SPRINGS CREEK

** Subtotal **

** FERC Number NV49556

ECCLES PLANT

THOUSAND SPRINGS CREEK

** Subtotal ** $\begin{array}{lll}\text { Dam } & \text { Name Plate } & \\ \text { ST Stat } & \text { Rating (KW) PESF } & \text { PESF * } \mathrm{KW}\end{array}$

$$
2000.00
$$

200.00

NV U

$1400.00 \quad 0.50$

700.00

1400.00

700.00

NV U

$128.00 \quad 0.90$

115.20

128.00

115.20

NV U

$65.00 \quad 0.90$

58.50

65.00

58.50

NV U

$115.00 \quad 0.90$

103.50

115.00

103.50

NV U

$76.00 \quad 0.90$

68.40

76.00

68.40

NV U

$76.00 \quad 0.90$

68.40

76.00

68.40 
Page No.

$08 / 19 / 97$

HYDROPOWER RESOURCE ASSESSMENT BY FERC NUMBER

Plant Name/

Stream

** FERC Number NVHOOO2

OVERTON PLANT

VIRGIN RIVER

** Subtotal **

** FERC Number NVHO004

CLEAR CREEK

CLEAR CREEK

** Subtotal **

** FERC Number NVH0006 BRYANT CREEK

BRYANT CREEK

** Subtotal **

** FERC Number NVHO008

SMITH VALLEY

DESERT CREEK

** Subtotal **

** FERC Number NVHO009

CHICKEN CREEK SUMMIT

JACK CREEK

** Subtotal **

** FERC Number NVH0011

ROWLAND PLANT

BRUNEAU RIVER

** Subtotal **

** FERC Number NVHO014 HOT SPRING CREEK PLANT MARY'S RIVER

\author{
Dam \\ ST Stat \\ Name Plate \\ Rating (KW) PESF
}

$\mathrm{PESF}$ * KW

NV U

$430.00 \quad 0.50$

215.00

430.00

215.00

NV U

$198.00 \quad 0.25$

49.50

198.00

49.50

NV U

$104.00 \quad 0.50$

52.00

104.00

52.00

NV U

$427.00 \quad 0.50$

213.50

427.00

213.50

NV U

$120.00 \quad 0.25$

30.00

120.00

30.00

NV U

$617.00 \quad 0.10$

61.70

617.00

61.70

NV U

$56.00 \quad 0.50$

28.00 

Plant Name/
Stream

** Subtotal **

** FERC Number NVH0015

HERDER PLANT

HERDER CREEK

** Subtotal **

** FERC Number NVHO016

TENT MOUNTAIN \#1

EAST FORK BOULDER CREEK

** Subtotal **

** FERC Number NVH0017

TENT MOUNTAIN \#2

WEST FORK BOULDER CREEK

** Subtotal **

** FERC Number NVH0018

SECRET PLANT

SECRET CREEK

** Subtotal **

** FERC Number NVH0019

TENT MOUNTAIN \#3

ACKLER CREEK

** Subtotal **

** FERC Number NVHO021

TALBOT PLANT

TALBOT CREEK

** Subtotal **

$$
56.00
$$

28.00

NV U

$126.00 \quad 0.90$

113.40

126.00

113.40

NV U

$343.00 \quad 0.90$

308.70

343.00

308.70

NV U

$301.00 \quad 0.90$

270.90

301.00

270.90

NV U

$301.00 \quad 0.90$

270.90

301.00

270.90

NV U

$203.00 \quad 0.90$

182.70

203.00

$182 \cdot 70$

NV U

$382.00 \quad 0.25$

95.50

382.00

95.50 


\section{Plant Name/}

stream

** FERC Number NVH0022

RYNDON PLANT

HUMBOLDT RIVER

** Subtotal **

** FERC Number NVH0023

THORPE PIANT

THORPE CREEK

** Subtotal **

** FERC Number NVHO026

ADOBIE PLANT

SUSIE CREEK

** Subtotal **

** FERC Number NVH0027

MAGGIE NARROWS

MAGGIE CREEK

** Subtotal **

** FERC Number NVHO028

OVERLAND PLANT

OVERLAND CREEK

** Subtotal **

** FERC Number NVHO029

FRANKLIN LAKE PLANT

FRANKLIN RIVER

** Subtotal **

** FERC Number NVHO031

MOUNT BARCROFT \#1

PERRY-AIKEN CREEK

\author{
Dam Name Plate \\ ST Stat Rating (KW) PESF PESF * KW
}

NV U

$200.00 \quad 0.50$

100.00

200.00

100.00

NV U

$244.00 \quad 0.90$

219.60

244.00

219.60

NV U

$$
49.00 \quad 0.90
$$

44.10

49.00

44.10

NV U

$710.00 \quad 0.25$

177.50

710.00

177.50

NV U

$323.00 \quad 0.90$

290.70

323.00

290.70

NV U

$230.00 \quad 0.50$

115.00

230.00

115.00

NV U

$313.00 \quad 0.90$

281.70 
Page No.

$08 / 19 / 97$

8

HYDROPOWER RE'SOURCE ASSESSMENT BY FERC NUMBER

Plant Name/

Stream

** Subtotal **

** FERC Number NVH0033

INDIAN CREEK PLANT

INDIAN CREEK

** Subtotal **

** FERC Number NVH0035

LEONARD RANCH PLANT

LEONARD CREEK

** Subtotal **

** FERC Number NVH0036

TRIDENT PLANT

KING'S RIVER

** Subtotal **

** FERC Number NVH0037

LOG CABIN PLANT

LOG CABIN CREEK

** Subtotal **

** FERC Number NVHO038

GRANITE PLANT

GRANITE CREEK

** Subtotal **

** FERC Number NVH0039

MCDERMITT \#1

EAST QUINN RIVER

** Subtotal **

$$
313.00
$$

NV U

$167.00 \quad 0.90$

150.30

167.00

150.30

NV U

$96.00 \quad 0.90$

86.40

96.00

86.40

NV U

$54.00 \quad 0.90$

48.60

54.00

48.60

NV U

$$
43.00 \quad 0.90
$$

38.70

$$
43.00
$$

38.70

NV U

$$
29.00 \quad 0.90
$$

$$
29.00
$$

NV U

$136.00 \quad 0.50$

68.00

136.00

68.00 
HYDROPOWER RESOURCE ASSESSMENT BY FERC NUMBER

\section{Plant Name/ stream}

** FERC Number NVH0040 MCDERMITT \#2 MCDERMITT CREEK

** Subtotal **

** FERC Number NVH0043

PARADISE \# 1

BIG COTTONWOOD CREEK

** Subtotal **

** FERC Number NVHO044

PARADISE \#2

INDIAN CREEK

** Subtotal **

** FERC Number NVHO045 GOLCONDA PLANT

POLE CREEK

** Subtotal **

** FERC Number NVHO046 ROCK CREEK DAM

ROCK CREEK

** Subtotal **

** FERC Number NVHOO48 URSINE PLANT

MEADOW VALLEY WASH

** Subtotal **

** FERC Number NVHOO49

EAST FORK POWER PLANT

EAST FORK WALKER RIVER

$\begin{array}{ll}\text { Dam } & \text { Name Plate } \\ \text { ST Stat } & \text { Rating (KW) PESF }\end{array}$

NV U

$62.00 \quad 0.90$

55.80

$$
62.00
$$

55.80

NV U

$102.00 \quad 0.90$

91.80

102.00

91.80

NV U

$183.00 \quad 0.50$

91.50

183.00

91.50

NV U

$73.00 \quad 0.90$

65.70

73.00

65.70

NV U

137.00

0.25

34.25

137.00

34.25

NV U

117.00

0.25

29.25

117.00

29.25

NV U

$108.00 \quad 0.50$

54.00 


\section{Plant Name/ Stream}

** Subtotal **

** FERC Number NVH0051

YOMBA PIJANT

REESE RIVER

** Subtotal **

** FERC Number NVH0052

ARC DOME \#1

SOUTH TWIN RIVER

** Subtotal **

** FERC Number NVH0053

CURRANT PLANT

CURRANT CREEK

** Subtotal **

** FERC Number NVH0055

WHITE'S PLANT

WHITE'S CREEK

** Subtotal **

** FERC Number NVH0063

GOSHUTE PLANT

SPRING CREEK

** Subtotal **

** FERC Number NVH0065

BAKER PLANT

BAKER CREEK

** Subtotal **

\author{
Dam Name Plate \\ ST stat Rating (KW) PESF \\ PESF * $\mathrm{KW}$
}

$$
108.00
$$

54.00

NV U

$89.00 \quad 0.50$

44.50

89.00

44.50

NV U

$162.00 \quad 0.50$

81.00

162.00

81.00

NV U

$81.00 \quad 0.90$

72.90

$$
81.00
$$

72.90

NV U

$213.00 \quad 0.90$

191.70

213.00

191.70

NV U

$64.00 \quad 0.50$

32.00

64.00

32.00

NV U

$161.00 \quad 0.90$

144.90

161.00

144.90 
HYDROPOWER RESOURCE ASSESSMENT BY FERC NUMBER

Plant Name/ Stream

** FERC Number NVH0068 MOTT CANYON PLANT MOTT CREEK

** Subtotal **

** FERC Number NVHO069 MOUNT SEIGEL PLANT

PINE NUT CREEK

** Subtotal **

** FERC Number NVHO071

B.C. PLANT

COON CREEK

** Subtotal **

** FERC Number NVH0072 COPPER MOUNTAIN PLANT COPPER CREEK

** Subtotal **

** FERC Number NVH0073

BIG BUCK PLANT

BUCK CREEK

** Subtotal **

** FERC Number NVH0074

DAVE POWER PLANT

DAVE CREEK

** Subtotal **

** FERC Number NVH0075 JARBIDGE \#1

EAST FORK JARBIDGE RIVER

\author{
Dam Name Plate \\ ST stat Rating (KW) PESF \\ PESF * KW
}

NV U

$163.00 \quad 0.90$

146.70

163.00

146.70

NV U

$839.00 \quad 0.25$

209.75

839.00

209.75

NV U

$102.00 \quad 0.25$

25.50

102.00

25.50

NV U

$58.00 \quad 0.50$

29.00

58.00

29.00

NV U

$197.00 \quad 0.10$

19.70

197.00

19.70

NV U

$91.00 \quad 0.10$

9.10

91.00

9.10

NV U

$260.00 \quad 0.10$

26.00 
HYDROPOWER RE'SOURCE ASSESSMENT BY FERC NUMBER

\section{Plant Name/ stream}

** Subtotal **

** FERC Number NVH0076

CANYON PLANT

CANYON CREEK

** Subtotal **

** FERC Number NVH0080

DIXIE FLATS PLANT

DIXIE CREEK

** Subtotal **

** FERC Number NVH0081

FRANKLIN \#3

MEYER'S CREEK

** Subtotal **

** FERC Number NVH0085

TRIDENT \#2

WILDER CREEK

** Subtotal **

** FERC Number NVH0089

UPPER SOLDIER MEADOWS

COLEMAN CREEK

** Subtotal **

** FERC Number NVH0092

SUMMIT PLANT

SUMMER CAMP CREEK

NV U

Dam

ST stat

NV U

Name Plate

Rating (KW) PESF

PESF * KW

260.00

26.00

10.20

102.00

10.20

NV U

$99.00 \quad 0.90$

89.10

99.00

89.10

21.50

43.00

21.50

NV U

$75.00 \quad 0.90$

67.50

75.00

67.50

NV U

$69.00 \quad 0.90$

$62 \cdot 10$

69.00

62.10

NV U

$67.00 \quad 0.90$

60.30

** Subtotal ** 


$$
\begin{gathered}
\text { Plant Name/ } \\
\text { Stream }
\end{gathered}
$$

** FERC Number NVH0093 BATTLE CREEK PLANT BATTLE CREEK

** Subtotal **

** FERC Number NVH0095 WILLOW CREEK PLANT BIG CREEK

** Subtotal **

** FERC Number NVH0096 REBEL YELL PLANT

REBEL CREEK

** Subtotal **

** FERC Number NVH0097 COLONY CREEK PLANT SINGAS CREEK

** Subtotal **

** FERC Number NVH0098 PARADISE \#2

WASH O'NEIL CREEK

** Subtotal **

** FERC Number NVH0099 STONE HOUSE PLANT ABEL CREEK

** Subtotal **

** FERC Number NVH0100 COLEMAN CREEK PLANT MULLINEX CREEK

\author{
Dam Name Plate \\ ST Stat Rating (KW) PESF \\ PESF * KW
}

NV U

$262.00 \quad 0.90$

235.80

262.00

235.80

NV U

$130.00 \quad 0.90$

117.00

130.00

117.00

NV U

$67.00 \quad 0.50$

33.50

67.00

33.50

NV U

$52.00 \quad 0.90$

46.80

52.00

46.80

NV U

$53.00 \quad 0.90$

47.70

53.00

47.70

NV U

$49.00 \quad 0.90$

44.10

49.00

44.10

NV U

$106.00 \quad 0.90$

95.40 
Plant Name/ stream

** Subtotal **

** FERC Number NVH0101

AUSTIN-50 PLANT

BIG CREEK

** Subtotal **

** FERC Number NVH0102

SPENCER PLANT

BIRCH CREEK

** Subtotal **

** FERC Number NVH0103

MILLET PLANT

BOWMAN CREEK

** Subtotal **

** FERC Number NVH0105

CALLAGHAN \#2

STEINER CREEK

** Subtotal **

** FERC Number NVH0106

PINE GROVE PLANT

DAIZELL CREEK

** Subtotal **

** FERC Number NVH0108

BODIE PLANT

BODIE CREEK

** Subtotal ** $\begin{array}{lll}\text { Dam } & \text { Name Plate } & \\ \text { ST Stat } & \text { Rating (KW) PESF } & \text { PESF * KW }\end{array}$

106.00

95.40

NV U

$63.00 \quad 0.50$

31.50

63.00

31.50

NV U

$94.00 \quad 0.50$

47.00

94.00

47.00

NV U

$45.00 \quad 0.50$

22.50

45.00

22.50

NV U

$110.00 \quad 0.90$

99.00

110.00

99.00

NV U

$793.00 \quad 0.50$

396.50

793.00

396.50

NV U

$854.00 \quad 0.90$

768.60

854.00

768.60 
Plant Name/

Stream

** FERC Number NVH0109

ROUGH WATER PLANT

ROUGH CREEK

** subtotal **

** FERC Number NVH0110

COTTONWOOD

COTTONWOOD CREEK

** Subtotal **

** FERC Number NVH0112

CORRAL WASH PLANT

CLEAR CREEK

** Subtotal **

** FERC Number NVH0115

PABLO CANYON PLANT

PABLO CANYON CREEK

** Subtotal **

** FERC Number NVH0116

BARKER PLANT

BARKER CREEK

** Subtotal **

** FERC Number NVH0117

NOT P.C. PLANT

BROAD CREEK

** Subtotal **

** FERC Number NVH0118

COVE PLANT

COVE CANYON CREEK

\author{
Dam Name Plate \\ ST Stat Rating (KW) PESF PESF * $\mathrm{KW}$
}

NV U

$470.00 \quad 0.90$

423.00

470.00

423.00

NV U

$1372.00 \quad 0.50$

686.00

1372.00

686.00

NV U

$41.00 \quad 0.50$

20.50

41.00

20.50

NV U

$95.00 \quad 0.50$

47.50

95.00

47.50

NV U

$69.00 \quad 0.90$

62.10

69.00

62.10

NV U

$104.00 \quad 0.50$

52.00

104.00

52.00

NV U

$67.00 \quad 0.50$

33.50 
Page No.

16

$08 / 19 / 97$

HYDROPOWER RESOURCE ASSESSMENT BY FERC NUMBER

Plant Name/

Stream

** Subtotal **

** FERC Number NVH0119 ARC DOME \#2

NORTH TWIN RIVER

** Subtotal **

** FERC Number NVH0120

LAST CHANCE PLANT

LAST CHANCE CREEK

** Subtotal **

** FERC Number NVH0121

GOLDRUSH PLANT

OPHIR CREEK

** Subtotal **

** FERC Number NVH0122

TEXTILE PLANT

DANVILLE CREEK

** Subtotal **

** FERC Number NVH0123

WINDOW PLANT

CLEAR CREEK

** Subtotal **

** FERC Number NVH0124

SOUTH SIXMILE CANYON

SIXMILE CREEK

** Subtotal **

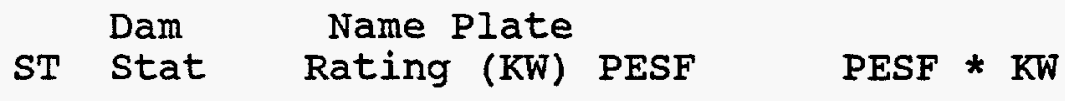

$$
67.00
$$

NV U

$134.00 \quad 0.50$

67.00

134.00

67.00

NV U

$78.00 \quad 0.50$

39.00

78.00

39.00

NV U

$78.00 \quad 0.50$

39.00

78.00

39.00

NV U

$96.00 \quad 0.50$

48.00

96.00

48.00

NV U

$82.00 \quad 0.50$

41.00

82.00

41.00

NV U

$106.00 \quad 0.90$

95.40

106.00

95.40 
Plant Name/

Stream

** FERC Number NVH0125

CARSON CITY

ASH CANYON CREEK

** Subtotal **

** FERC Number NVH0126

UNIONVILLE \#2

INDIAN CREEK

** Subtotal **

** FERC Number NVH0127

UNIONVILLE \#3

COTTONWOOD CREEK

** Subtotal **

** FERC Number NVH0128

UNIONVILLE \#1

BUENA VISTA CREEK

** Subtotal **

** FERC Number NVH0129

BASQUE PLANT

EDWARDS CREEK

** Subtotal **

** FERC Number NVH0133

DIAMOND PEAK PLANT

INCLINE CREEK

** Subtotal **

** FERC Number NVH0134

GREY POWER PLANT

WEST FORK GREY CREEK

\author{
Dam Name Plate \\ ST Stat Rating (KW) PESF PESF * KW
}

NV U

$244.00 \quad 0.90$

219.60

244.00

219.60

NV U

$43.00 \quad 0.90$

38.70

43.00

38.70

NV U

$43.00 \quad 0.90$

38.70

43.00

38.70

NV U

$79.00 \quad 0.90$

71.10

79.00

71.10

NV U

$94.00 \quad 0.90$

84.60

94.00

84.60

NV U

$110.00 \quad 0.90$

99.00

110.00

99.00

NV U

$189.00 \quad 0.90$

170.10 
Plant Name/ stream

** Subtotal **

** FERC Number NVH0136

MOUNT ROSE PLANT WEST

DEEP CANYON

** Subtotal **

** FERC Number NVH0137

UNNAMED PLANT

(NO NAME)

** Subtotal **

** FERC Number NVH0138

BIG INDIAN PLANT

BIG INDIAN CREEK

** Subtotal **

** FERC Number NVH0139

KALAMAZOO PLANT

KALAMAZOO CREEK

** Subtotal **

** FERC Number NVH0142

BIG WASH PLANT

BIG WASH CREEK

** Subtotal **

** FERC Number NVH0143

SNAKE PLANT

SNAKE CREEK

** Subtotal ** $\begin{array}{ll}\text { Dam } & \text { Name Plate } \\ \text { ST Stat } & \text { Rating (KW) PESF }\end{array}$

$$
189.00
$$

170.10

NV U

$427.00 \quad 0.50$

213.50

427.00

213.50

NV U

$305.00 \quad 0.90$

274.50

305.00

274.50

NV U

$79.00 \quad 0.50$

39.50

79.00

39.50

NV U

$211.00 \quad 0.50$

105.50

211.00

105.50

NV U

$185.00 \quad 0.50$

92.50

185.00

92.50

NV U

$165.00 \quad 0.50$

82.50

165.00

82.50 
Plant Name/

stream

** FERC Number NVH0144 PRIOR TO PRESTON PLANT WHITE RIVER

** Subtotal **

** FERC Number NVU0001 PATSVILLE OWYHEE RIVER

** Subtotal **

** FERC Number NVU0002 SKULL CREEK OWYHEE RIVER

** Subtotal **

** FERC Number NVU0017 DEVIL'S GATE RESERVOIR NORTH FORK HUMBOLDT RIVER

** Subtotal **

** FERC Number NVU0018 EUREKA DAM

CARSON RIVER

** Subtotal **

** FERC Number NVU0020

HOYE CANYON

WEST WALKER RIVER

** Subtotal **

** FERC Number NVU0023 KEYSTONE RESERVOIR GLEASON CREEK

$\begin{array}{lll}\text { Dam } & \text { Name Plate } & \\ \text { ST Stat } & \text { Rating (KW) PESF } & \text { PESF * KW }\end{array}$

NV U

$1057.00 \quad 0.90$

951.30

1057.00

951.30

NV U

$1600.00 \quad 0.10$

160.00

1600.00

160.00

NV U

$3400.00 \quad 0.10$

340.00

3400.00

340.00

NV U

$1900.00 \quad 0.90$

1710.00

1900.00

1710.00

NV U

$6000.00 \quad 0.25$

1500.00

6000.00

1500.00

NV U

$3400.00 \quad 0.50$

1700.00

3400.00

1700.00

NV U

$1200.00 \quad 0.90$

1080.00 
Plant Name/ stream

** Subtotal **

** FERC Number 01746

LEIDY CR (CIRCLE L)

LEIDY $C R$

** Subtotal **

** FERC Number 04164

WASHOE

TRUCKEE R

** Subtotal **

** FERC Number 06420

SWEETWATER RANCH

SWEETWATER CREEK

** Subtotal **

** FERC Number NV01412

OPHIR POWER PLANT

OPHIR CREEK

** Subtotal **

** FERC Number NV03720

CURLEY-WOOD

JARBIDGE RIVER

** Subtotal **

** FERC Number NV06850

BOWERS MANSION PLANT

FRANKTOWN CREEK

** Subtotal **

$\begin{array}{lll}\text { Dam } & \text { Name Plate } & \\ \text { ST Stat } & \text { Rating (KW) PESF } & \text { PESF * KW }\end{array}$

$$
1200.00
$$

1080.00

NV $W$

$200.00 \quad 0.75$

150.00

200.00

150.00

NV W

$900.00 \quad 0.90$

810.00

900.00

810.00

NV W

$319.00 \quad 0.50$

159.50

319.00

159.50

NV W

$335.00 \quad 0.75$

251.25

335.00

251.25

NV W

305.00

0.50

152.50

305.00

152.50

NV W

$811.00 \quad 0.90$

729.90

811.00

729.90 
HYDROPOWER RESOURCE ASSESSMENT BY FERC NUMBER

\section{Plant Name/ \\ Stream}

** FERC Number NV09344

PECETTI

THOMAS CREEK

** Subtotal **

** FERC Number NV80000

26 FOOT DROP POWER PLANT

$\mathrm{V}$ CANAL

** Subtotal **

** FERC Number NVH0042

PARADISE MILI

MARTIN CREEK

** Subtotal **

** FERC Number 05602A

ALDER CREEK 1

ALDER CREEK

** Subtotal **

** FERC Number 05619

KNOTT CREEK

KNOTT CREEK

** Subtotal **

** FERC Number 08392

RYE PATCH

HUMBOLDT RIVER

** Subtotal **

** FERC Number 08703

NEVADA STATE SUPPLY

NV ST CONDUIT (MARLETTE LK) $\begin{array}{ll}\text { Dam } & \text { Name Plate } \\ \text { ST } & \text { Stat } \\ \text { Rating (KW) PESF }\end{array}$

PESF * KW

NV W

$142.00 \quad 0.90$

127.80

142.00

127.80

NV W

$1600.00 \quad 0.90$

1440.00

1600.00

1440.00

NV W

$126.00 \quad 0.75$

94.50

126.00

94.50

NV wo

$1300.00 \quad 0.90$

1170.00

1300.00

1170.00

NV wo

$554.00 \quad 0.75$

415.50

554.00

415.50

NV wo

$1400.00 \quad 0.75$

1050.00

1400.00

1050.00

NV wo

$1100.00 \quad 0.75$

825.00 
Plant Name/ Stream

** Subtotal **

** FERC Number 09530

TAHOE TUNNEL

LAKE TAHOE EFFLUENT PIPELINE

** Subtotal **

** FERC Number 09618

ROBINSON SPRINGS

ROBINSON SPRINGS

** Subtotal **

** FERC Number 10958

LAS VEGAS WASH

LAS VEGAS WASH

** Subtotal **

** FERC Number 11311A

TS RANCH HYDRO 1

GOLD MINE DISCHARGE PIPELINE

** Subtotal **

** FERC Number 11311B

TS RANCH HYDRO 2

GOLD MINE DISCHARGE PIPELINE

** Subtotal **

** FERC Number NV00005

ONION RESERVOIR

IITTLE ALDER CREEK

NV wo

NV wo

NV wo

NV wo

NV wo

NV wo

$$
1100.00
$$

825.00

$2500.00 \quad 0.75$

1875.00

2500.00

1875.00

$300.00 \quad 0.75$

225.00

300.00

225.00

$2780.00 \quad 0.75$

2085.00

2780.00

2085.00

$2500.00 \quad 0.75$

1875.00

2500.00

1875.00

$2000.00 \quad 0.75$

1500.00

2000.00

1500.00

57.00

$76.00 \quad 0.75$

57.00

** Subtotal **

57.00 
HYDROPOWER RESOURCE ASSESSMENT BY FERC NUMBER

Plant Name/

Stream

** FERC Number NV00016

LITTLE VALLEY RESERVOIR

COLEMAN CANYON CREEK

** Subtotal **

** FERC Number NV00022

WALL CANYON \#1

WALI CREEK

** Subtotal **

** FERC Number NV00023

WALI CANYON \#2

WALI CREEK

** Subtotal **

** FERC Number NV00024

BILK CREEK RESERVOIR

BIIK CREEK

** Subtotal **

** FERC Number NV00037

BULL RUN RESERVOIR

BULL RUN CREEK

** Subtotal **

** FERC Number NV00050

METROPOLIS RESERVOIR

BISHOP CREEK

** Subtotal **

** FERC Number NV00051

BOYD RESERVOIR

RABBIT CREEK $\begin{array}{ll}\text { Dam } & \text { Name Plate } \\ \text { Stat } & \text { Rating (KW) PESF }\end{array}$

NV wo

$$
29.00 \quad 0.90
$$

26.10

29.00

26.10

NV wo

$1100.00 \quad 0.90$

990.00

1100.00

990.00

NV wo

$600.00 \quad 0.90$

540.00

$$
600.00
$$

540.00

NV wo

$185.00 \quad 0.90$

166.50

185.00

166.50

NV wo

$160.00 \quad 0.90$

144.00

160.00

144.00

NV wo

$1800.00 \quad 0.90$

1620.00

1800.00

1620.00

NV wo

$250.00 \quad 0.90$

225.00 


\section{Plant Name/ stream}

** Subtotal **

** FERC Number NV00055 ZUNINO RESERVOIR SMITH CREEK

** Subtotal **

** FERC Number NV00063 LOWER PITT-TAYLOR RES. HUMBOIDT RIVER

** Subtotal **

** FERC Number NV00109 DAEK RESERVOIR THOUSAND SPRINGS CREEK

** Subtotal **

** FERC Number NV00110 TWENTY THREE MILE RES. THOUSAND SPRINGS CREEK

** Subtotal **

** FERC Number NV00112

SILVER CREEK DAM

SILVER CREEK

** Subtotal **

** FERC Number NV00113

BOWMAN RESERVOIR

MUDDY RIVER

** Subtotal ** $\begin{array}{lll}\text { Dam Name Plate } & \\ \text { ST Stat } & \text { Rating (KW) PESF } & \text { PESF * KW }\end{array}$

$$
250.00
$$

225.00

NV wo

$107.00 \quad 0.75$

80.25

$$
107.00
$$

80.25

NV wo

$1500.00 \quad 0.90$

1350.00

1500.00

1350.00

NV wo

$1300.00 \quad 0.90$

1170.00

1300.00

1170.00

NV wo

$1100.00 \quad 0.90$

990.00

1100.00

990.00

NV wo

$311.00 \quad 0.90$

279.90

311.00

279.90

NV wo

$284.00 \quad 0.90$

255.60

284.00

255.60 
Plant Name/

stream

** FERC Number NV00127

GROVES LAKE

KINGSTON CREEK

** Subtotal **

** FERC Number NV00174

COMINS LAKE

STEPTOE CREEK

** Subtotal **

** FERC Number NV00191

SEYLER RESERVOIR

PEAVINE CREEK

** Subtotal **

** FERC Number NV00197

SOLDIER MEADOWS RESERVOIR

SOLDIER CREEK

** Subtotal **

** FERC Number NV00199

INCLINE LAKE

THIRD CREEK

** Subtotal **

** FERC Number NV00226

SOUTH FORK RESERVOIR

SOUTH FORK HUMBOLDT RIVER

** Subtotal **

** FERC Number NV00234

EAST PEAK LAKE

DAGGETT CREEK

$\begin{array}{llll} & \text { Dam } & \text { Name Plate } & \\ \text { ST Stat } & \text { Rating (KW) PESF } & \text { PESF * KW }\end{array}$

NV wo

$225.00 \quad 0.75$

168.75

225.00

168.75

NV wo

$102.00 \quad 0.75$

76.50

102.00

76.50

NV wo

$53.00 \quad 0.90$

47.70

53.00

47.70

NV wo

$116.00 \quad 0.90$

104.40

116.00

104.40

NV wo

$422.00 \quad 0.75$

316.50

422.00

316.50

NV wo

$2900.00 \quad 0.75$

2175.00

2900.00

2175.00

NV wo

$131.00 \quad 0.90$

117.90 
Plant Name/ Stream

** Subtotal **

** FERC Number NV01151

CHIMNEY RESERVOIR

LITTLE HUMBOLDT RIVER

** Subtotal **

** FERC Number NV01198

FLORISTON MILL

BRONCO AND DRY PRARIE CREEKS

** Subtotal **

** FERC Number NV03898

ROUND MOUNTAIN

JETT CREEK

** Subtotal **

** FERC Number NV10101

CAT CREEK RESERVOIR

CAT CREEK

** Subtotal **

** FERC Number NV10114

PALISADES \#2

PINE CREEK

** Subtotal **

** FERC Number NV10120

CARSON RIVER DIVERSION

CARSON RIVER

** Subtotal **

$\begin{array}{lll} & \text { Dam } & \text { Name Plate } \\ \text { ST Stat } & \text { Rating (KW) PESF } & \text { PESF * KW }\end{array}$

$$
131.00
$$

117.90

NV Wo

$95.00 \quad 0.75$

71.25

95.00

71.25

NV Wo

$427.00 \quad 0.90$

384.30

427.00

384.30

NV wo

$82.00 \quad 0.90$

73.80

82.00

73.80

NV wo

$630.00 \quad 0.50$

315.00

630.00

315.00

NV WO

$77.00 \quad 0.90$

69.30

77.00

69.30

NV WO

$2200.00 \quad 0.75$

1650.00

2200.00

1650.00 
Plant Name/

Stream

** FERC Number NV10121

DERBY DIVERSION DAM

TRUCKEE RIVER

** Subtotal **

** FERC Number NV10132

WEBER DAM

WALKER RIVER

** Subtotal **

** FERC Number NV10133

STILLWATER POINT

CARSON RIVER

** Subtotal **

** FERC Number NV10170

LOGAN CREEK

LOGAN CREEK

** Subtotal **

** FERC Number NV10189

HEADGATE DAM

SALMON FALLS CREEK

** Subtotal **

** FERC Number NV10222

$B-B$ MINE RESERVOIR

ROCK CREEK

** Subtotal **

** FERC Number NV10223

CHIATOVICH CREEK RESERVOIR

CHIATOVICH CREEK

$\begin{array}{ll}\text { Dam } & \text { Name Plate } \\ \text { ST Stat } & \text { Rating (KW) PESF } \quad \text { PESF * KW }\end{array}$

NV wo

$2300.00 \quad 0.50$

1150.00

2300.00

1150.00

NV wo

1700.00

0.75

1275.00

1700.00

1275.00

NV wo

1100.00

0.10

110.00

1100.00

110.00

NV wo

203.00

0.75

152.25

203.00

152.25

NV WO

270.00

0.75

202.50

270.00

202.50

NV WO

285.00

0.90

256.50

285.00

256.50

NV wo

$177.00 \quad 0.90$

159.30 
Page No.

28

$08 / 19 / 97$

HYDROPOWER RESOURCE ASSESSMENT BY FERC NUMBER

Plant Name/

Stream

** Subtotal **

** FERC Number NV10322

RATTLESNAKE RESERVOIR

QUEEN CREEK

** Subtotal **

** FERC Number NV10460

CALLAGHAN CREEK RESERVOIR

CALLAGHAN CREEK

** Subtotal **

** FERC Number NV10470

TOPAZ RESERVOIR

WALKER RIVER

** Subtotal **

** FERC Number NV10472

WASHOE LAKE

STEAMBOAT CREEK

** Subtotal **

** FERC Number NVH0083

QUEEN RANCH PLANT

MORRIS CREEK

** Subtotal **

*** Total *** $\begin{array}{lll}\text { Dam } & \text { Name Plate } & \\ \text { ST Stat } & \text { Rating (KW) PESF } & \text { PESF * KW }\end{array}$

177.00

159.30

NV wo

$140.00 \quad 0.90$

126.00

140.00

126.00

NV wo

$83.00 \quad 0.90$

74.70

83.00

74.70

NV wo

$4000.00 \quad 0.75$

3000.00

4000.00

3000.00

NV wo

$69.00 \quad 0.75$

51.75

69.00

51.75

NV wo

$142.00 \quad 0.50$

71.00

142.00

71.00

125908.00

67225.95 
Appendix D

Individual Resource Database List 

R E S O U R C E
D A T A B A $S E$
L I S T I N G

DATE: $08 / 19 / 97$

PAGE NO: 1

FERC

Number

Plant Name

Stream

State

Name

01746

LEIDY CR (CIRCLE I)

LEIDY CR

NV

county Name

River Basin

ESMERALDA

FISH LAKE BASIN

Class Owner Name

$\mathrm{R}$ FOUR RENT INC

Name Plate

Rating (KW)

PESF

Annual Energy

PESF*KW Rating (MWh)

PESF Annual

$200.00 \quad 0.75 \quad 150.00$

650.00

487.50

Unit

Plant

Project

Dam

Type

Type

status

status

Latitude $\quad 3744.00$

C

ROR

XX

W

Factor

Exist Prob

Factor

Exist Prob

wild/Scenic Protection

Wild/Scenic Tributary or

0.90 Wildlife Value

Threatened/Endangered Fish

Upstream/Downstream Threatened/Endangered Wildlife wild/Scenic Location

$\begin{array}{ll}0.90 & \text { Federal Land code } 103 \\ 0.90 & \text { Federal Land Code } 104\end{array}$

0.90 Federal Land Code 105

0.90 Federal Land code 106

0.90 Federal Land code 107

Energy Rating (MWh)

Cultural Value

Fish Presence Value

Geologic Value

other value

Recreation Value

0.90

Federal Land Code 108

0.90

Federal Land Code 198

0.90 
R E S O U R C E D A T A B A S E I I S T I N G

DATE: $08 / 19 / 97$

PAGE NO: 2

FERC

Number

Plant Name

stream

state

Name

04164 WASHOE

TRUCKEE R

NV

county Name

River Basin

WASHOE

TRUCKEE RIVER BASIN

Class Owner Name

$P$ SIERRA PACIFIC POWER CO

Name Plate

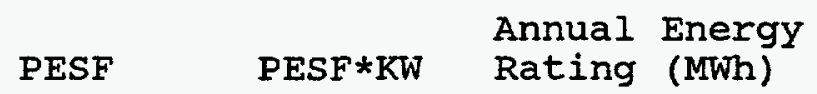

PESF Annual

Rating (KW)

$0.90 \quad 810.00$

3200.00

Energy Rating

(MWh)

900.00

Unit Plant

Project

Dam

status

Latitude

Longitude 11956.00

2880

Type

Type

status

W

Factor

Exist Prob

Factor

Exist Prob

0.90

Wildlife Value

Threatened/Endangered Fish

0.90

Wild/Scenic Protection

wild/Scenic Tributary or Threatened/Endangered Wildlife

0.90

Upstream/Downstream

wild/Scenic Location

0.90 Federal Land code 103

0.90

0.90 Federal Land Code 104

0.90

Cultural value

0.90 Federal Land Code 105

0.90

Fish Presence Value

0.90 Federal Land Code 106

0.90

0.90 Federal Land Code 107

0.90

Historic Value

0.90

Federal Land Code 108

0.90

other Value

Recreation Value

0.90

Federal Land code 198

0.90

Scenic Value

0.90

0.90 
DATE: $08 / 19 / 97$

FERC

Number

Plant Name

05423A LAS VEGAS WASH 2

county Name

CIARK

Class Owner Name

R HYDRO ELEC CONSTRUCTION INC

Name Plate

Rating (KW)

PESF

Annual Energy

400.00

$0.90 \quad 360.00$

PESF*KW

Rating (MWh)

3500.00

Unit Plant

Type

Type

C

DIV

Factor

Exist Prob

0.90

Wildlife value Threatened/Endangered Fish

Wild/Scenic Protection
Wild/Scenic Tributary or

Upstream/Downstream

wild/Scenic Location

Cultural value

Fish Presence Value

Geologic Value

Historic Value

other value

Recreation Value

Scenic Value $\begin{array}{llr}\text { Dam } & \text { Latitude } & 3606.00 \\ \text { Status } & \text { Longitude } & 11450.00\end{array}$

$\mathrm{U}$
0.90 Federal Land Code 103

0.90 Federal Land Code 104

0.90 Federal Land Code 105

0.90 Federal Land Code 106

0.90 Federal Land Code 107

0.90 Federal Land Code 108

0.90 Federal Land Code 198

0.90
PAGE NO: 3

State

Name

NV
PESF Annual

Energy Rating (MWh)

3150

Exist Prob

0.90

0.90

0.90

0.90

0.90

0.90

0.90

0.90

0.90

0.90 

R E S O U R C E
D A T A B A S E
I I S T I N G

DATE : $08 / 19 / 97$

PAGE NO: 4

FERC

Number

Plant Name

Stream

state

05423B LAS VEGAS WASH 3

IAS VEGAS WASH

Name

O5423B LAS VECAS WASH 3

County Name

River Basin

CLARK

COLORADO RIVER BASIN

$\begin{array}{cc}\text { Class } & \text { Owner Name } \\ R & \text { HYDRO ELEC CONSTRUCTION INC }\end{array}$

Name Plate

Rating ( $\mathrm{KW}$ )

PESF

Annual Energy

$300.00 \quad 0.90 \quad 270.00$

PESF*KW

Rating (MWh)

PESF Annual

Energy Rating (MWh)

NV

\section{0}

$\begin{array}{ccccc}\text { Unit } & \text { Plant } & \text { Project } & \text { Dam } & \text { Latitude } \\ \text { Type } & \text { Type } & \text { Status } & \text { Status } & \text { Longitude } 11501.00 \\ \text { C } & \text { DIV } & \mathrm{XX} & \mathrm{U} & \end{array}$

Factor

Exist Prob

Factor

Exist Prob

wild/Scenic Protection

Wild/Scenic Tributary or

0.90 Wildlife Value

Threatened/Endangered Fish

0.90

Threatened/Endangered Wildlife

0.90

Upstream/Downstream

wild/Scenic Location

Cultural value

Fish Presence Value

$\begin{array}{ll}0.90 & \text { Federal Land Code } 103 \\ 0.90 & \text { Federal Land Code } 104\end{array}$

0.90

$\begin{array}{ll}0.90 & \text { Federal Land Code } 104 \\ 0.90 & \text { Federal Land Code } 105\end{array}$

0.90

0.90 Federal Land Code 106

0.90

0.90

Geologic Value

$\begin{array}{ll}0.90 & \text { Federal Land Code } 106 \\ 0.90 & \text { Federal Land Code } 107\end{array}$

0.90

Historic Value

0.90 Federal Land Code 108

0.90

other Value

Recreation Value

0.90 Federal Land Code 198

0.90

0.90

0.90

Scenic Value 

R E S O U R C E
D A T A B A S E
I I S T I N G

DATE : $08 / 19 / 97$

PAGE NO: 5

FERC

Number

Plant Name

stream

State

Name

05602A ALDER CREEK 1

ALDER CREEK

NV

county Name

River Basin

HUMBOLDT

CONTINENTAL LAKE BASIN

Class Owner Name

$R$ LIVE OAK ASSOC WEST

Name Plate

$\begin{array}{ll}\text { PESF } & \text { PESF*KW } \\ \text { Rating (MWh) }\end{array}$

PESF Annual

Rating (KW)

$0.90 \quad 1170.00$

4500.00

Energy Rating (MWh)

1300.00

recot

Dam

status

Latitude $\quad 4141.00$

Type

Type

status

Longitude 11846.00

c

STR

$\mathrm{xx}$

wo

Factor

Exist Prob

Factor

Exist Prob

Wild/Scenic Protection

Wild/Scenic Tributary or

0.90 Wildife Value

Threatened/Endangered Fish

Upstream/Downstream

wild/Scenic Location

Cultural value

Fish Presence Value

Geologic Value

Historic Value

Other Value

Recreation value

Scenic Value

\section{Threatened/Endangered Wildlife}

0.90 Federal Land code 103

0.90 Federal Land Code 104

0.90 Federal Land Code 105

0.90 Federal Land Code 106

0.90 Federal Land Code 107

0.90 Federal Land Code 108

0.90 Federal Land Code 198
0.90

0.90

0.90

0.90

0.90

0.90

0.90

0.90

0.90

0.90 
FERC

Number

Plant Name

05602B ALDER CREEK 2

County Name

HUMBOLDT stream

ALDER CREEK

River Basin

CONTINENTAL LAKE BASIN
State

Name

NV

Class Owner Name

R LIVE OAK ASSOC WEST

Name Plate

Rating ( $\mathrm{KW}$ )

PESF

Annual Energy

275.00

$0.90 \quad 247.50$

1500.00

PESF Annual

Energy Rating (MWh)

1350

$\begin{array}{ccccc}\text { Unit } & \text { Plant } & \text { Project } & \text { Dam } & \text { Latitude } \\ \text { Type } & \text { Type } & \text { Status } & \text { Status } & \text { Longitude } 11845.00 \\ \text { C } & \text { ROR } & \text { XX } & \text { U } & \end{array}$

Factor

Wild/Scenic Protection

wild/Scenic Tributary or Upstream/Downstream wild/Scenic Location Cultural value Fish Presence Value Geologic Value Historic Value other Value Recreation Value Scenic Value
Exist Prob

0.90

Wildife Value Threatened/Endangered Fish Threatened/Endangered Wildlife

0.90 Federal Land Code 103

0.90 Federal Land Code 104

0.90 Federal Land Code 105

0.90 Federal Land Code 106

0.90 Federal Land Code 107

0.90 Federal Land Code 108

0.90 Federal Land Code 198
Exist Prob

0.90

0.90

0.90

0.90

0.90

0.90

0.90

0.90

0.90

0.90 
DATE : 08/19/97

FERC

Number

Plant Name

05619 KNOTT CREEK

County Name

HUMBOLDT
PAGE NO: 7

State

Name

KNOTT CREEK

NV
River Basin

GRIDLEY LAKE BASIN

Class Owner Name

$R \quad R$ D D INC

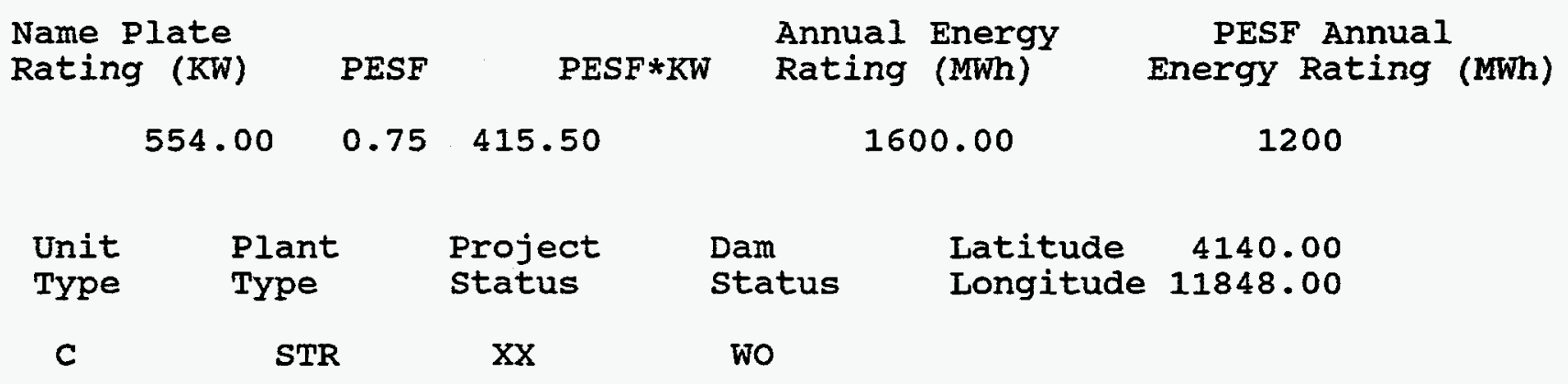

Factor

Exist Prob

Factor

Exist Prob

Wild/scenic Protection

Wild/Scenic Tributary or

Upstream/Downstream

wild/Scenic Location

Cultural value

Fish Presence Value

Geologic Value

Historic Value

other Value

Recreation value

Scenic Value

0.90 Wildife Value
Threatened/Endangered Fish

Threatened/Endangered Wildlife

0.90 Federal Land Code 103

0.90 Federal Land Code 104

0.90 Federal Land Code 105

0.90 Federal Land Code 106

0.90 Federal Land Code 107

0.90 Federal Land Code 108

0.90 Federal Land Code 198

0.90
0.90

0.90

0.90

0.90

0.75

0.90

0.90

0.90

0.90

0.90 
R E S O U R C E D A T A B A S E I I S T I N G

DATE: $08 / 19 / 97$

PAGE NO: 8

FERC

Number

Plant Name

Stream

State

Name

06133 BROKEN DAM

EAST FORK CARSON RIVER

NV

County Name

DOUGLAS
River Basin

CARSON RIVER BASIN
Class Owner Name

R SFRINGER, $M \&$ BOULDEN, $J$

Name Plate

Rating (KW)

PESF

Annual Energy

$\mathrm{PESF} * \mathrm{KW}$

Rating (MWh)

PESF Annual

2000.00

$0.10 \quad 200.00$

12600.00

1260

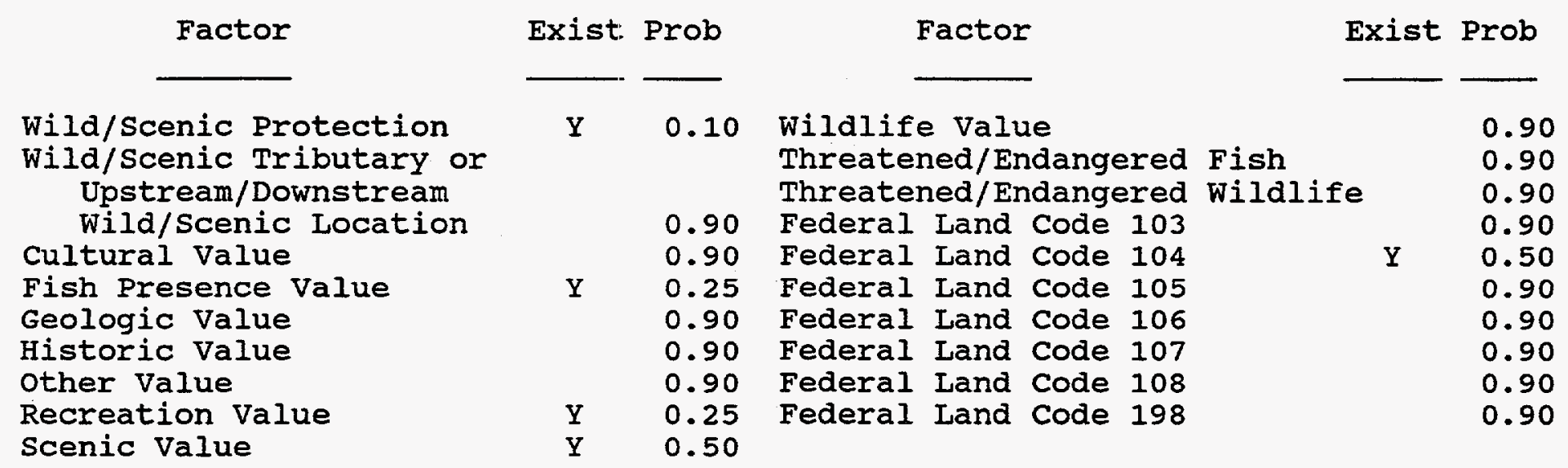


DATE : $08 / 19 / 97$

FERC

Number

06420 SWEETWATER RANCH

County Name

LYON

Class

R

SWEETWATER RANCH CO

Name Plate

Rating (KW)

PESF

Annual Energy

Rating (MWh)

$$
319.00 \quad 0.50 \quad 159.50
$$

PESF*KW

1189.00

Dam

Unit Plant

Type

Type

C

ROR
Project status

$\mathrm{xx}$
River Basin

WALKER LAKE BASIN
PAGE NO: 9

Stream

state

Name

NV
PESF Annual Energy Rating (MWh)

594.50

Factor

Exist Prob

Factor

Exist Prob

Wild/Scenic Protection

Wild/Scenic Tributary or

Upstream/ Downstream

wild/Scenic Location

Cultural Value

Fish Presence Value

Geologic Value

Historic Value

other Value

Recreation Value

Scenic Value

\begin{tabular}{|c|c|}
\hline & 0.90 \\
\hline & 0.90 \\
\hline$Y$ & 0.75 \\
\hline $\bar{Y}$ & 0.75 \\
\hline & $\begin{array}{l}0.90 \\
0.90\end{array}$ \\
\hline$Y$ & 0.75 \\
\hline$Y$ & 0.75 \\
\hline$Y$ & 0.90 \\
\hline
\end{tabular}

Wildife Value

Threatened/Endangered Fish Threatened/Endangered Wildlife

Federal Land Code 103

Federal Land code 104

Federal Land Code 105

Federal Land code 106

Federal Land Code 107

Federal Land Code 108

Federal Land Code 198
0.90

0.90

0.90

0.90

0.75

0.90

0.90

0.90

0.90

0.90 
R E S O URC E D A T A B A S E L I S T I N G

DATE: $08 / 19 / 97$

PAGE NO: 10

FERC

Number

Plant Name

06457 IAMOILLE

county Name

ELKO

class Owner Name

R BROWN, KENT L

Name Plate

Rating (KW)

1600.00

PESF

PESF*KW

Annual Energy

Rating (MWh)

6200.00

$0.25 \quad 400.00$

Plant

Type

Project status

C

ROR

$x x$

Dam
status

Latitude $\quad 4039.00$

Type

Factor

Exist Prob

Factor

Exist Prob

Wild/Scenic Protection

Wild/Scenic Tributary or Upstream/Downstream wild/Scenic Location

Cultural value

Fish Presence Value

Geologic Value

Historic Value

other Value

Recreation Value

Scenic Value

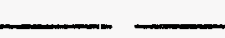

$$
0.90
$$

Wildlife Value

Threatened/Endangered $\mathrm{Fish}$

Threatened/Endangered wildlife

0.90 Federal Land Code 103

0.90 Federal Land Code 104

0.90 Federal Land Code 105

0.90 Federal Iand code 106

0.90 Federal Land Code 107

0.90 Federal Land Code 108

$\mathrm{Y} \quad 0.25$ Federal Land Code 198

$\begin{array}{ll}\mathrm{Y} & 0.25 \\ \mathrm{Y} & 0.50\end{array}$
State Name

NV
PESF Annual Energy Rating (MWh)
1550

$\mathrm{U}$

0.90

0.90

0.90

0.90

0.90

0.90

0.90

0.90

0.90

0.90 
DATE: $08 / 19 / 97$

FERC

Number

07392

BUSH RANCH
Plant Name

County Name

ELKO
PAGE NO: 11

State

Name

BISHOP CREEK

NV

River Basin

HUMBOLDT RIVER BASIN

Class Owner Name

R BUSH, I F

Name Plate

Rating (KW)

163.00
Annual Energy

PESF*KW

Rating (MWh)

855.00
PESF Annual Energy Rating (MWh)

769.50

\begin{tabular}{|c|c|c|c|}
\hline $\begin{array}{l}\text { Unit } \\
\text { Type }\end{array}$ & $\begin{array}{l}\text { Plant } \\
\text { Type }\end{array}$ & $\begin{array}{l}\text { Project } \\
\text { status }\end{array}$ & $\begin{array}{l}\text { Dam } \\
\text { status }\end{array}$ \\
\hline
\end{tabular}

C ROR

$\mathrm{XX}$

U

Factor

Exist Prob

Factor

Exist Prob

Wild/Scenic Protection

wild/Scenic Tributary or

0.90 Wildlife Value

Threatened/Endangered Fish

Upstream/Downstream wild/Scenic Location Threatened/Endangered Wildlife

0.90 Federal Land Code 103

0.90

0.90

Federal Land code 104

0.90

0.90

Federal Land Code 105

0.90

Cultural Value

Fish Presence Value

0.90

Federal Land Code 106

0.90

0.90

Federal Land Code 107

0.90

Historic Value

0.90

Federal Land Code 108

0.90

other Value

Recreation Value

0.90

0.90

0.90

Federal Land Code 198

0.90

0.90

0.90 

R E S O U R C I
D A T A B A S E
L I S T I N G

DATE: $08 / 19 / 97$

PAGE NO: 12

FERC

Number

Plant Name

07507 SOUTH FORK

county Name

ELRO

River Basin

HUMBOLDT RIVER BASIN

$\begin{array}{cr}\text { Class } & \text { Owner Name } \\ \text { R } & \text { BROWN, KENT L }\end{array}$

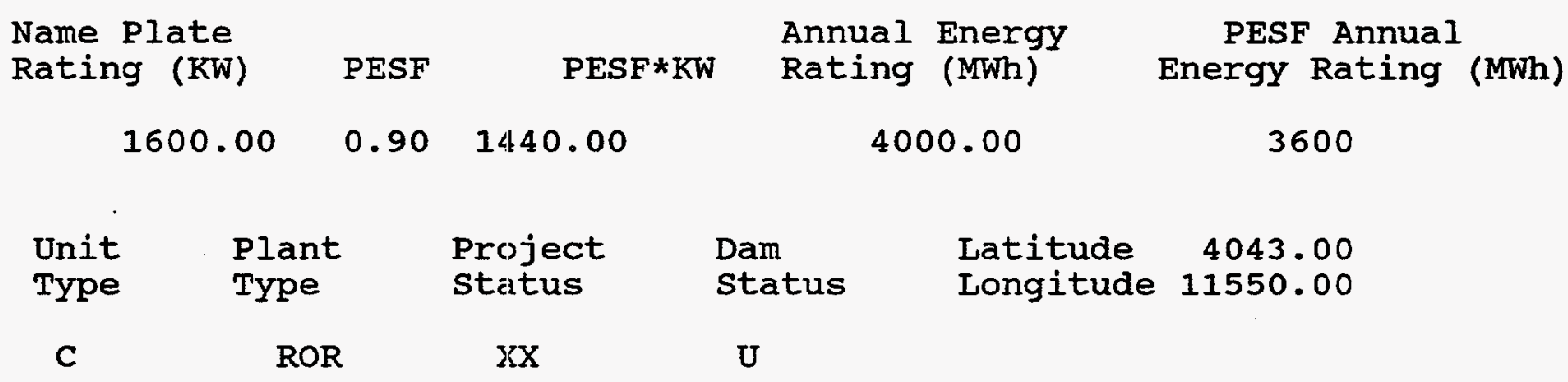

Factor

Exist: Prob

0.90 Wildlife Value

Threatened/Endangered Fish

wild/Scenic Tributary or Upstream/Downstream wild/Scenic Location Cultural value

Fish Presence Value

Geologic Value

Historic Value

other Value

Recreation Value

Scenic Value
Factor

Exist Prob
State

Name

NV

SOUTH FORK HUMBOLDT RIVER

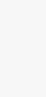


R E S O U R C E

DATE: $08 / 19 / 97$

FERC

Number

Plant Name

07779 GALENA CREEK

county Name

WASHOE

Class

$\mathbf{R}$
D A T A B A S E

L I S T I G

PAGE NO: 13 state

Name

GALENA CREEK

NV
River Basin

TRUCKEE RIVER BASIN

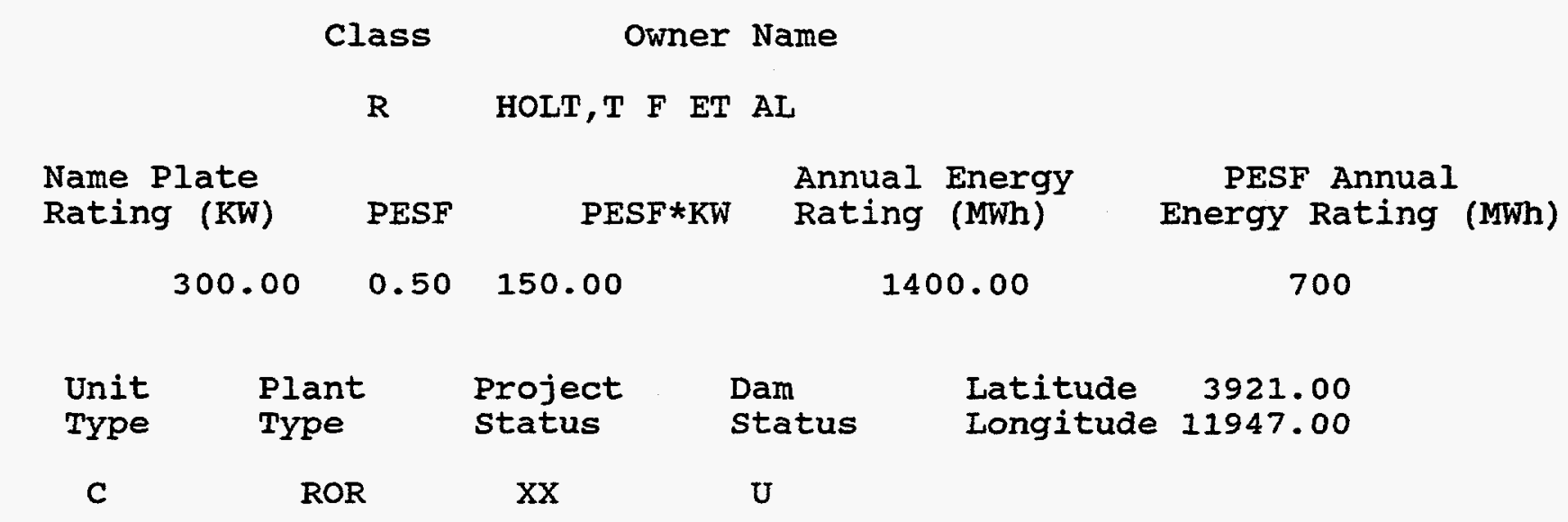

Factor

wild/Scenic Protection

wild/Scenic Tributary or

Upstream/Downstream

wild/Scenic Location

Cultural value

Fish Presence Value

Geologic Value

Historic Value

other value

Recreation value

Scenic Value
Exist Prob

Factor

Exist Prob

0.90 Wildlife Value

Threatened/Endangered Fish Threatened/Endangered Wildlife

0.90 Federal Land Code 103

0.90 Federal Land Code 104

0.90 Federal Land code 105

0.90 Federal Iand Code 106

0.90 Federal Land Code 107

0.90 Federal Land Code 108

0.90 Federal Land Code 198

0.90 
DATE: $08 / 19 / 97$

PAGE NO: 14

\section{FERC}

Number

Plant Name

08392 RYE PATCH

County Name

PERSHING

River Basin

HUMBOLDT RIVER BASIN

HUMBOLDT RIVER

State

Name

NV

Class Owner Name

R ROCKY MOUNTAIN HYDRO INC

Name Plate

Rating ( $\mathrm{KW}$ )

PESF

Annual Energy

1400.00

$0.75 \quad 1050.00$

5500.00

PESF Annual

Energy Rating (MWh)

4125

\begin{tabular}{|c|c|c|c|c|}
\hline $\begin{array}{l}\text { Unit } \\
\text { Type }\end{array}$ & $\begin{array}{l}\text { Plant } \\
\text { Type }\end{array}$ & $\begin{array}{l}\text { Project } \\
\text { status }\end{array}$ & $\begin{array}{l}\text { Dam } \\
\text { status }\end{array}$ & $\begin{array}{lr}\text { Latitude } & 4028.00 \\
\text { Longitude } & 11818.00\end{array}$ \\
\hline C & STR & $2 x X$ & wo & \\
\hline
\end{tabular}

Factor

Exist Prob

Factor

Exist Prob

Wild/scenic Protection

Wild/Scenic Tributary or

0.90 Wildlife Value
Threatened/Endangered Fish

Threatened/Endangered Wildlife

Upstream/Downstream

0.90 Federal Land Code 103

0.90 Federal Land Code 104

0.90 Federal Land Code 105

0.90 Federal Land Code 106

0.90 Federal Land Code 107

0.90 Federal Land Code 108

0.90 Federal Land Code 198

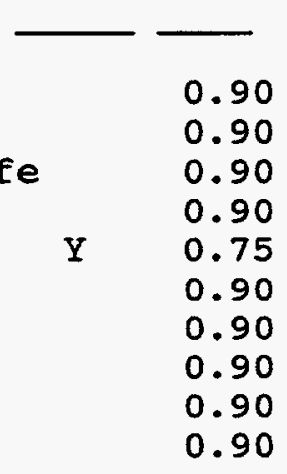

Recreation Value

0.90

Scenic Value 

R E S O U R C E
D A T A B A S E
I I S I N G

DATE: $08 / 19 / 97$

PAGE NO: 15

FERC

Number

08703

Plant Name

NEVADA STATE SUPPLY

County Name

WASHOE

Class

Owner Name

R MEGA RENEWABLES

Name Plate

Rating (KW)

PESF

Annual Energy

PESF*KW

Rating (MWh)

PESF Annual

1100.00

$0.75 \quad 825.00$

9000.00

Energy Rating (MWh)

6750

\begin{tabular}{|c|c|c|c|c|}
\hline $\begin{array}{l}\text { Unit } \\
\text { Type }\end{array}$ & $\begin{array}{l}\text { Plant } \\
\text { Type }\end{array}$ & $\begin{array}{l}\text { Project } \\
\text { Status }\end{array}$ & $\begin{array}{l}\text { Dam } \\
\text { status }\end{array}$ & $\begin{array}{lr}\text { Latitude } & 3911.00 \\
\text { Longitude } & 11948.00\end{array}$ \\
\hline C & DIV & $X X$ & wo & \\
\hline
\end{tabular}

Factor

Exist Prob

Factor

Exist Prob

Wild/scenic Protection

wild/Scenic Tributary or

Upstream/Downstream wild/Scenic Location

Cultural value

Fish Presence Value

Geologic Value

Historic Value

other Value

Recreation Value

Scenic Value

0.90 Wildlife Value

Threatened/Endangered Fish

Threatened/Endangered Wildlife

0.90 Federal Land Code 103

0.90 Federal Land Code 104

0.90 Federal Land Code 105

0.90 Federal Land Code 106

0.90 Federal Land Code 107

0.90 Federal Land Code 108

0.90 Federal Land Code 198

0.90
State

Name

NV 
R E S U R C E D A T A B A S I I S T I N

DATE: $08 / 19 / 97$

PAGE NO: 16

FERC

Number

Plant Name

Stream

State

08973 TRUCKEE

TRUCKEE RIVER

Name

NV

county Name

STOREY
River Basin

TRUCKEE RIVER BASIN

class Owner Name

R ROCKY MOUNTAIN HYDRO INC

Name Plate

Rating ( $\mathrm{KW})$

PESF

Annual Energy

PESF Annual

$1500.00 \quad 0.50$

$0.50 \quad 750.00$

6500.00

Energy Rating (MWh)

$\begin{array}{lll}\text { Unit } & \text { Plant } & \text { Project } \\ \text { Type } & \text { Type } & \text { Sta.tus }\end{array}$

C ROR XX

$\begin{array}{llr}\text { Dam } & \text { Latitude } & 3934.00 \\ \text { Status } & \text { Longitude } & 11922.00\end{array}$

$\mathrm{U}$

Factor

Wild/Scenic Protection

Wild/scenic Tributary or Upstream/Downstream wild/Scenic Location Cultural Value

Fish Presence Value

Geologic Value

Historic Value

other Value

Recreation value

Scenic Value
Exist. Prob

0.90

Wildlife value Threatened/Endangered Fish Threatened/Endangered Wildlife

0.90 Federal Land Code 103

0.90 Federal Land Code 104

0.90 Federal Land Code 105

0.90 Federal Land code 106

0.90 Federal Land Code 107

0.90 Federal Land Code 108

0.90 Federal Land Code 198

0.90
3250

Exist Prob

0.90

0.90

0.90

0.90

Y

0.50

0.90

0.90

0.90

0.90

0.90 
DATE: $08 / 19 / 97$

FERC

Number

09530

TAHOE TUNNEL

County Name

DOUGLAS

Class

Owner Name

R DOUGLAS HYDRO INC

Name Plate

Rating (KW)

PESF

Annual Energy

$P E S F * K W$

Rating (MWh)

17500.00

PAGE NO: 17

LAKE TAHOE EFFLUENT PIPELINE

River Basin

LAKE TAHOE BASIN

$$
2500.00 \quad 0.75 \quad 1875.00
$$

state

Name

NV

Project

status

Dam

Type

Type

$\mathrm{XX}$

Status

Latitude 3858.00

c

DIV

WO

Factor

Wild/Scenic Protection

Wild/Scenic Tributary or Upstream/Downstream wild/Scenic Location

Cultural Value

Fish Presence value

Geologic Value

Historic Value

other Value

Recreation Value

Scenic Value
Exist Prob

Factor

Exist Prob

0.90

Wildlife Value

Threatened/Endangered Fish

Threatened/Endangered Wildlife

0.90 Federal Land Code 103

0.90 Federal Land Code 104

0.90 Federal Land Code 105

0.90 Federal Land Code 106

0.90 Federal Land Code 107

0.90 Federal Land Code 108

0.90 Federal Land Code 198

0.90
PESF Annual Energy Rating (MWh)

13125 
DATE: $08 / 19 / 97$

FERC

Number

Plant Name

09618 ROBINSON SPRINGS

County Name

WHITE PINE

Class

Owner Name

R HARRIS, BILL

Name Plate

Rating (KW)

PESF

Annual Energy

$\mathrm{PESF} * \mathrm{KW}$

Rating (MWh)

1500.00

$0.75 \quad 225.00$

300.00

Project

status

XX

Exist Prob

Factor

Latitude $\quad 3938.00$

Type

Type

ROR

Factor

0.90

Wildlife Value

Threatened/Endangered Fish

Threatened/Endangered Wildlife

0.90 Federal Land Code 103

0.90 Federal Land Code 104

0.90 Federal Land Code 105

0.90 Federal Land Code 106

0.90 Federal Land Code 107

0.90 Federal Land Code 108

0.90 Federal Land Code 198

0.90
Fish Presence Value

Geologic Value

Historic Value

other Value

Recreation value

Scenic Value
Wild/Scenic Protection Upstream/Downstream wild/Scenic Location
State

Name

NV
PESF Annual Energy Rating (MWh)

1125 

R E S O U R C E
D A T A B A S E
L I S T I N G

DATE: $08 / 19 / 97$

PAGE NO: 19

FERC

Number

Plant Name

Stream

state

09646 HUNTER CREEK

HUNTER CREEK

Name

County Name

River Basin

WASHOE

TRUCKEE RIVER BASIN

$\begin{array}{cc}\text { Class } & \text { Owner Name } \\ R & \text { HUNTER CREEK HYDRO CO }\end{array}$

Name Plate

Rating (KW)

PESF

Annual Energy

PESF Annual

270.00

$0.90 \quad 243.00$

855.00

769.50

\begin{tabular}{|c|c|c|c|c|}
\hline $\begin{array}{l}\text { Unit } \\
\text { Type }\end{array}$ & $\begin{array}{l}\text { Plant } \\
\text { Type }\end{array}$ & $\begin{array}{l}\text { Project } \\
\text { status }\end{array}$ & $\begin{array}{l}\text { Dam } \\
\text { status }\end{array}$ & $\begin{array}{lr}\text { Latitude } & 3929.00 \\
\text { Longitude } & 11953.00\end{array}$ \\
\hline C & ROR & $x x$ & $U$ & \\
\hline
\end{tabular}

Factor

Exist Prob

0.90

Wildife Value

Threatened/Endangered Fish

Threatened/Endangered Wildlife

0.90 Federal Land Code 103

0.90 Federal Land Code 104

0.90 Federal Land code 105

0.90 Federal Land Code 106

0.90 Federal Land Code 107

0.90 Federal Land code 108

0.90 Federal Land Code 198

0.90
Wild/scenic Tributary or wild/scenic Location

Fish Presence Value

Geologic Value

other Value

Recreation Value

Scenic Value
Exist Prob

0.90

0.90

0.90

0.90

0.90

0.90

0.90

0.90

0.90

0.90 
DATE: $08 / 19 / 97$

PAGE NO: 20

FERC

Number

Plant Name

stream

State

10463 S NEVADA TREATMENT

IAS VEGAS EFFLUENT

Name

NV

county Name

River Basin

CLARK

COLORADO RIVER BASIN

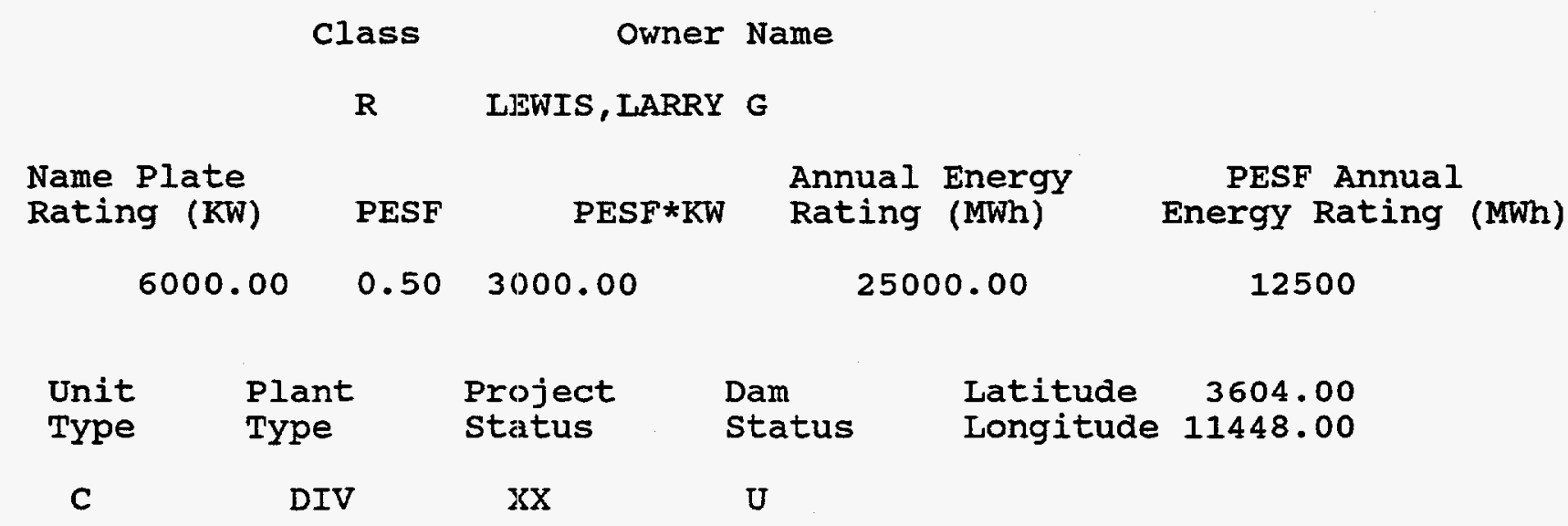

Factor

Wild/Scenic Protection

wild/Scenic Tributary or

Upstream/Downstream

wild/scenic Location

Cultural value

Fish Presence Value

Geologic Value

Historic Value

other Value

Recreation Value

Scenic Value
Exist Prob

0.90

Wildife value

Threatened/Endangered Fish

Threatened/Endangered Wildlife

0.90 Federal Land code 103

0.90 Federal Land Code 104

0.90 Federal Land Code 105

0.90 Federal Land Code 106

0.90 Federal Land Code 107

0.90 Federal Land Code 108

0.90 Federal Land Code 198

0.90
Exist Prob

0.90

0.90

0.90

0.90

$\mathbf{Y}$

0.90

0.90

0.90

0.90

0.90 
DATE: $08 / 19 / 97$

PAGE NO: 21

FERC

Number

10577
Plant Name

WETLANDS RECOVEROS CONTRL

County Name

CLARK

Class

Owner Name

$R$ LEWIS, LARRY $G$

Name Plate

Rating (KW)

3150.00

PESF
Annual Energy
$\mathrm{PESF} * \mathrm{KW}$
Rating (MWh)

17500.00

0.501575 .00

Plant Project

Type

status

$\mathrm{XX}$

Status

Latitude $\quad 3606.00$

Type

DIV

C

Factor

Exist Prob

Factor

Exist Prob

Wild/scenic Protection

wild/Scenic Tributary or

Upstream/Downstream wild/Scenic Location

Cultural value

Fish Presence Value

Geologic Value

Historic Value

other Value

Recreation Value

Scenic Value
0.90 Wildlife value

Threatened/Endangered Fish

Threatened/Endangered Wildlife

0.90 Federal Land Code 103

0.90 Federal Land code 104

0.90 Federal Land code 105

0.90 Federal Land Code 106

0.90 Federal Land code 107

0.90 Federal Land code 108

0.90 Federal Land Code 198

0.90
PESF Annual
Energy Rating (MWh)
8750

State

Name

NV 
R E S O U R C E D A T A B A S E I I S T I N G

DATE: $08 / 19 / 97$

PAGE NO: 22

FERC

Number

Plant Name

Stream

State

10958 LAS VEGAS WASH

county Name

LAS VEGAS WASH

NV

CLARK

River Basin

COLORADO RIVER BASIN

Class Owner Name

$R$ LAKE AT LAS VEGAS JNT VNTR

Name Plate

$\begin{array}{lll}\text { PESF } & \text { PESF*KW } & \begin{array}{l}\text { Annual Energy } \\ \text { Rating }\end{array} \\ \text { (MWh) }\end{array}$

PESF Annual

Rating (KW)

$0.75 \quad 2085.00$

11800.00

Energy Rating (MWh)

2780.00

8850

\begin{tabular}{|c|c|c|c|c|}
\hline $\begin{array}{l}\text { Unit } \\
\text { Type }\end{array}$ & $\begin{array}{l}\text { Plant } \\
\text { Type }\end{array}$ & $\begin{array}{l}\text { Project } \\
\text { status }\end{array}$ & $\begin{array}{l}\text { Dam } \\
\text { status }\end{array}$ & $\begin{array}{lr}\text { Latitude } \quad 3607.00 \\
\text { Longitude } 11454.00\end{array}$ \\
\hline C & ROR & $x x$ & wo & \\
\hline
\end{tabular}

Factor

Exist: Prob

Factor

Exist Prob

Wild/Scenic Protection

wild/Scenic Tributary or Upstream/Downstream wild/Scenic Location Cultural value

Fish Presence Value

Geologic Value

Historic Value

other value

Recreation value

Scenic Value
0.90

Wildife Value

Threatened/Endangered Fish

Threatened/Endangered Wildlife

0.90 Federal Land Code 103

0.90 Federal Land Code 104

0.90 Federal Land Code 105

0.90 Federal Land Code 106

0.90 Federal Land Code 107

$\mathrm{Y} \quad 0.75$

0.90

0.90
Federal Land Code 108

Federal Land Code 198
0.90

0.90

0.90

0.90

0.90

0.90

0.90

0.90

0.90

0.90 
DATE : $08 / 19 / 97$

FERC

Number

Plant Name

11311A TS RANCH HYDRO 1

County Name

EUREKA
PAGE NO: 23

State

Name

GOLD MINE DISCHARGE PIPELINE

NV
River Basin

HUMBOLDT RIVER BASIN $\begin{array}{cr}\text { Class } & \text { Owner Name } \\ \text { M } & \text { TS HYDRO }\end{array}$

Name Plate Rating ( $\mathrm{KW})$

2500.00

PESF

Annual Energy

PESF*KW

Rating (MWh)

19700.00

$0.75 \quad 1875.00$

Plant

Type

ROR

Project status

$\mathrm{XX}$

Exist Prob

Factor

Latitude $\quad 4058.00$

Dam

status

Longitude 11623.00
Factor

Wild/Scenic Protection

wild/Scenic Tributary or

Upstream/Downstream

wild/Scenic Location

Cultural value

Fish Presence Value

Geologic Value

Historic Value

other value

Recreation Value

Scenic Value

\subsection{Wildlife Value}

Threatened/Endangered Fish Threatened/Endangered Wildlife

0.90 Federal Land Code 103

0.90 Federal Land Code 104

0.90 Federal Land Code 105

0.90 Federal Land Code 106

0.90 Federal Land Code 107

0.90 Federal Land Code 108

0.90 Federal Land Code 198
PESF Annual Energy Rating (MWh)

14775

0.90 
DATE: $08 / 19 / 97$

FERC

Number Plant Name

11311B TS RANCH HYDRO 2

County Name

EUREKA

Class Owner Name

M T\$ HYDRO

Name Plate Rating (KW)

2000.00

PESF

Annual Energy Rating (MWh)

13000.00

0.751500 .00

stream

PAGE NO: 24

GOLD MINE DISCHARGE PIPELINE

River Basin

HUMBOLDT RIVER BASIN
State

Name

NV

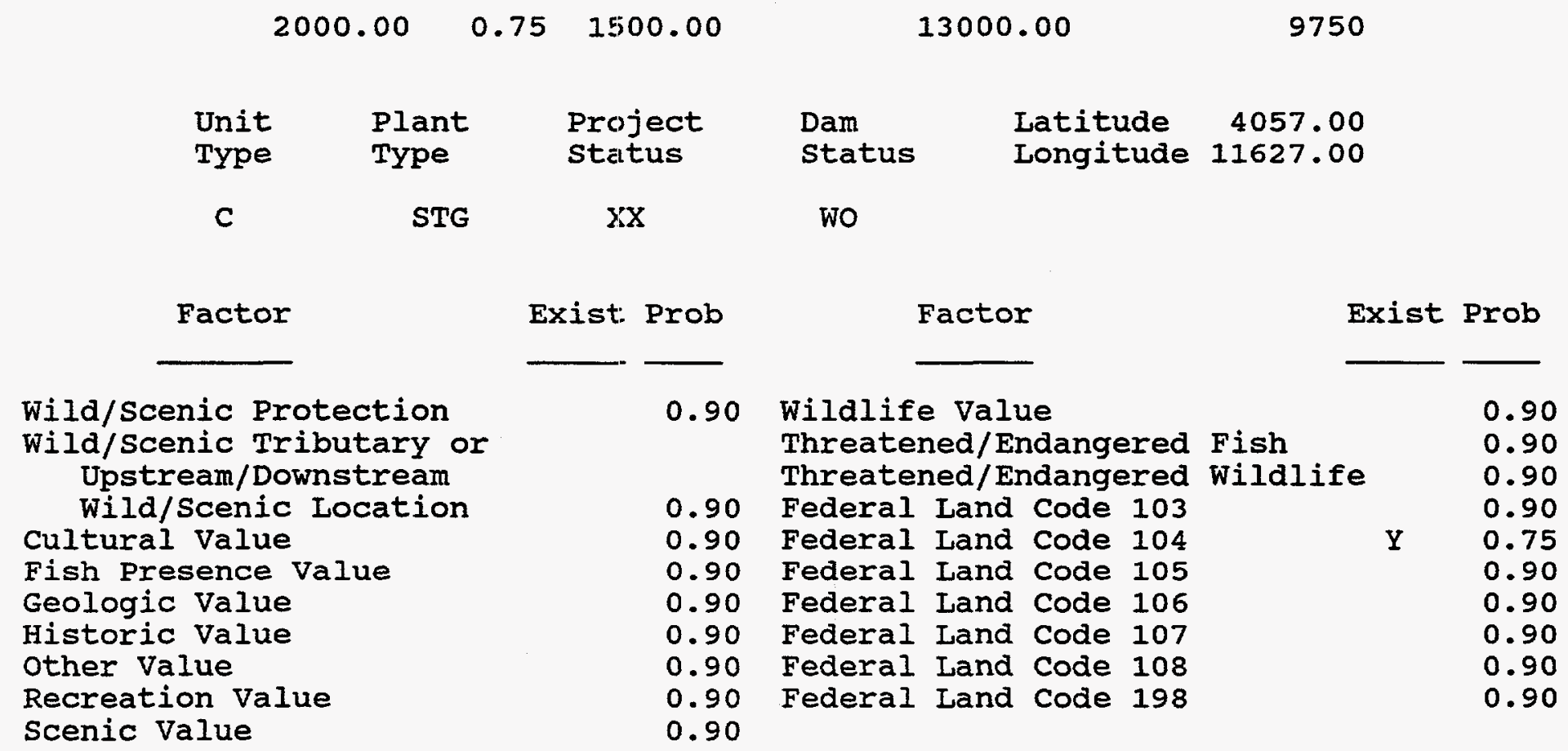



R E S O U R C E
D A T A B A $S E$
I I S T I N G

DATE: $08 / 19 / 97$

PAGE NO: 25

FERC

Number

Plant Name

NVO0005 ONION RESERVOIR

county Name

HUMBOLDT

\author{
stream \\ LITTLE ALDER CREEK \\ River Basin \\ CONTINENTAL LAKE BASIN
}

State

Name

NV

$\begin{array}{cc}\text { Class } & \text { Owner Name } \\ \text { R } & \text { NORTH FORK RANCH COMPANY }\end{array}$

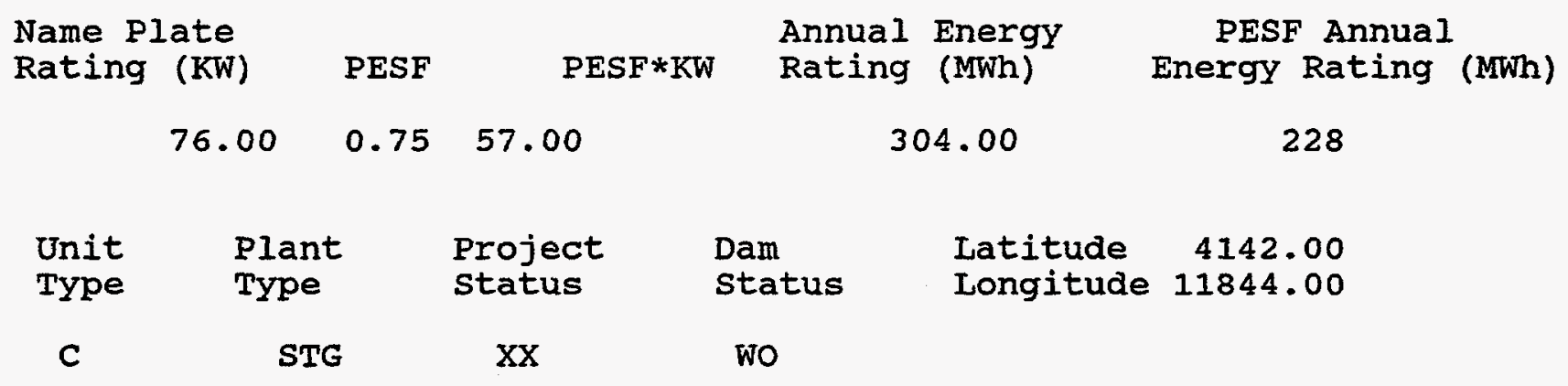

Factor

Exist Prob

Factor

Exist Prob

Wild/Scenic Protection

wild/Scenic Tributary or

Upstream/Downstream wild/Scenic Location Cultural value

Fish Presence Value

Geologic Value

Historic value

other Value

Recreation Value

Scenic Value

$\begin{array}{clll}0.90 & \begin{array}{l}\text { Wildife Value } \\ \text { Threatened/Endangered Fish }\end{array} & 0.90 \\ & & 0.90 \\ 0.90 & \text { Federal Land Code 103 } & 0.90 \\ 0.90 & \text { Federal Land Code 104 } & 0.90 \\ 0.90 & \text { Federal Land Code 105 } & 0.90 \\ 0.90 & \text { Federal Land Code 106 } & 0.90 \\ 0.90 & \text { Federal Land Code 107 } & 0.90 \\ 0.90 & \text { Federal Land Code 108 } & 0.90 \\ & 0.75 & \text { Federal Land code 198 } & 0.90 \\ & 0.90 & & 0.90\end{array}$


DATE: $08 / 19 / 97$

PAGE NO: 26

FERC

Number

Plant Name

stream

State

NV00016 LITTLE VALLEY RESERVOIR

COLEMAN CANYON CREER

Name

NV

county Name

WASHOE
River Basin

BIG LAKE BASIN

\section{Class Owner Name}

R UINKNOWN

\begin{tabular}{|c|c|c|c|c|c|c|}
\hline \multicolumn{2}{|c|}{$\begin{array}{l}\text { Name Plate } \\
\text { Rating (KW) }\end{array}$} & PESF & $\mathrm{PESF} * \mathrm{KW}$ & $\begin{array}{l}\text { Annu } \\
\text { Rati }\end{array}$ & $\begin{array}{ll}1 & \text { Energy } \\
g & (\mathrm{MWh})\end{array}$ & $\begin{array}{l}\text { PESF Annual } \\
\text { Energy Rating (MWh) }\end{array}$ \\
\hline & 29.00 & 0.90 & 26.10 & & 121.00 & 108.90 \\
\hline $\begin{array}{l}\text { Unit } \\
\text { Type }\end{array}$ & $\begin{array}{l}\text { Pla } \\
\text { Tyl }\end{array}$ & & $\begin{array}{l}\text { Project } \\
\text { Status }\end{array}$ & $\begin{array}{l}\text { Dam } \\
\text { status }\end{array}$ & $\begin{array}{l}\text { Latitude } \\
\text { Longitude }\end{array}$ & $\begin{array}{r}4155.00 \\
\quad 11945.00\end{array}$ \\
\hline C & & & $3 x$ & พo & & \\
\hline
\end{tabular}

Factor

Exist: Prob

0.90

Wild/scenic Protection

Wild/Scenic Tributary or Upstream/Downstream wild/Scenic Location Cultural value

Fish Presence Value Geologic Value

Historic Value

Other Value

Recreation Value

Scenic Value

0.90
Factor

Exist Prob

Wildlife Value Threatened/Endangered Fish Threatened/Endangered Wildlife

0.90 Federal Land Code 103

0.90 Federal Land Code 104

0.90 Federal Land Code 105

0.90 Federal Land Code 106

0.90 Federal Land Code 107

0.90 Federal Land Code 108

0.90 Federal Land code 198
0.90

0.90

0.90

0.90

0.90

0.90

0.90

0.90

0.90

0.90 
DATE: $08 / 19 / 97$

FERC

Number

Plant Name

NV00022 WALL CANYON \#1

county Name

WASHOE

Class Owner Name

R WESTERN FARM CREDIT BANK

Name Plate Rating (KW)

PESF

Annual Energy

PESF *KW Rating (MWh)

2500.00

Unit

Plant

Type

Type

c

STG

Factor

Exist Prob

0.90

wildlife Value

Threatened/Endangered Fish

Wild/Scenic Tributary or

Upstream/Downstream

wild/Scenic Location

Cultural Value

Fish Presence Value

Geologic Value

Historic Value

other Value

Recreation value

Scenic Value $\begin{array}{llr}\text { Dam } & \text { Latitude } & 4106.00 \\ \text { Status } & \text { Longitude } & 11951.00\end{array}$

wo
0.90 Federal Land Code 103

0.90 Federal Land Code 104

wildlife

0.90 Federal Land code 105

0.90 Federal Land Code 106

0.90 Federal Land Code 107

0.90 Federal Land Code 108

0.90 Federal Land Code 198

0.90
PAGE NO: 27

State

Name

NV
PESF Annual

Energy Rating (MWh)

2250

Exist Prob

Exist Prob 
R E S O U R C E D A T A B A S E I I S T I N G

DATE: $08 / 19 / 97$

PAGE NO: 28

FERC

Number

Plant Name

stream

State

NV00023 WALI CANYON \#2

WALL CREEK

Name

County Name

River Basin

WASHOE

SUPRISE BASIN

Class Owner Name

R WESTERN FARM CREDIT BANK

Name Plate

$\begin{array}{ll}\text { PESF } & \text { PESF*KW } \\ \text { Rating (MWh) }\end{array}$

PESF Annual

Rating (KW)

$0.90 \quad 540.00$

2000.00

Energy Rating (MWh)

600.00

Unit

Plant

Type

Project Status

Dam

Status

Latitude $\quad 4110.00$

Type

STG

$\mathrm{XX}$

wo

Factor

wild/scenic Protection

wild/Scenic Tributary or Upstream/ Downstream wild/scenic Location Cultural value

Fish Presence Value

Geologic Value

Historic Value

other value

Recreation Value

Scenic Value
Exist: Prob

0.90

Wildife value

Threatened/Endangered Fish

Threatened/Endangered Wildlife

0.90 Federal Land code 103

0.90 Federal Land code 104

0.90 Federal Land Code 105

0.90 Federal Land Code 106

0.90 Federal Land Code 107

0.90 Federal Land Code 108

0.90 Federal Land Code 198
NV

\section{0}


DATE: $08 / 19 / 97$

FERC

Number

NV00024 BILK CREEK RESERVOIR

county Name

HUMBOLDT
PAGE NO: 29

state

Name

NV
BILK CREEK

River Basin

BLACK ROCK DESERT BASIN

Class Owner Name

R C2 CATTLE COMPANY

\begin{tabular}{|c|c|c|}
\hline $\begin{array}{l}\text { Name Plate } \\
\text { Rating (KW) }\end{array}$ & PESF & PESF*KW \\
\hline
\end{tabular}

185.00

$0.90 \quad 166.50$

Unit

Type
Plant
Type
STG

c

\begin{abstract}
Project Status
\end{abstract}

$\mathrm{XX}$
679.00
PESF Annual Energy Rating (MWh)

611.10

Factor

Wild/Scenic Protection

wild/Scenic Tributary or Upstream/Downstream wild/Scenic Location

Cultural Value

Fish Presence Value

Geologic Value

Historic Value

other Value

Recreation Value

Scenic Value
Exist Prob

Factor

Latitude

4135.00

Status

Longitude 11825.00

wo
0.90 Wildife Value

Threatened/Endangered Fish Threatened/Endangered Wildlife

0.90 Federal Land Code 103

0.90 Federal Land Code 104

0.90 Federal Land Code 105

0.90 Federal Land Code 106

0.90 Federal Land code 107

0.90 Federal Land Code 108

0.90 Federal Land Code 198
Exist Prob

0.90

0.90

0.90

0.90

0.90

0.90

0.90

0.90

0.90

0.90 


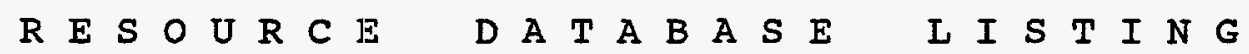

DATE: $08 / 19 / 97$

PAGE NO: 30

FERC

Number

Plant Name

NV00037 BULL RUN RESERVOIR

county Name

ELKO

stream

State

Name

BULL RUN CREEK

NV

River Basin

SNAKE RIVER BASIN

Class Owner Name

R PETAN COMPANY

Name Plate

Rating (KW)

PESF

Annual Energy

PESF Annual

160.00

$0.90 \quad 144.00$

579.00

521.10

Energy Rating (MWh)

\begin{tabular}{|c|c|c|c|c|}
\hline $\begin{array}{l}\text { Unit } \\
\text { Type }\end{array}$ & $\begin{array}{l}\text { Plant } \\
\text { Type }\end{array}$ & $\begin{array}{l}\text { Project } \\
\text { status }\end{array}$ & $\begin{array}{l}\text { Dam } \\
\text { status }\end{array}$ & $\begin{array}{lr}\text { Latitude } & 4138.00 \\
\text { Longitude } & 11609.00\end{array}$ \\
\hline C & STG & $\mathrm{xX}$ & พо & \\
\hline
\end{tabular}

Factor

Wild/Scenic Protection

wild/Scenic Tributary or Upstream/Downstream wild/scenic Location Cultural value Fish Presence Value Geologic Value Historic Value other Value Recreation value Scenic value
Exist: Prob

0.90

Wildlife Value

Threatened/Endangered Fish Threatened/Endangered Wildlife

0.90 Federal Land Code 103

0.90 Federal Land Code 104

0.90 Federal Land Code 105

0.90 Federal Land Code 106

0.90 Federal Land Code 107

0.90 Federal Land Code 108

0.90 Federal Land Code 198
Exist Prob

0.90

0.90

0.90

0.90

0.90

0.90

0.90

0.90

0.90

0.90 
DATE: $08 / 19 / 97$

FERC

Number

Plant Name

NV00050 METROPOLIS RESERVOIR

County Name

ELKO

Class

$\mathbf{R}$

PACIFIC RECLAMATION COMPANY

Name Plate Rating ( $\mathrm{KW}$ )

PESF

PESF*KW

Annual Energy Rating (MWh)

1800.00

$0.90 \quad 1620.00$

Plant

Type

STG

C

Unit
Type

Project Status

$\mathrm{xx}$

Exist Prob

Factor

Latitude $\quad 4115.00$

Dam

Status

Longitude 11455.00

wo
Factor

wild/Scenic Protection

wild/scenic Tributary or

Upstream/Downstream

wild/Scenic Location

Cultural value

Fish Presence Value

Geologic Value

Historic Value

other Value

Recreation value

Scenic Value
PAGE NO: 31

State

Name

NV
PESF Annual Energy Rating (MWh)

2700

$\begin{array}{lll}0.90 & \text { Wildlife Value } & 0.90 \\ & \text { Threatened/Endangered Fish } & 0.90 \\ & \text { Threatened/Endangered Wildlife } & 0.90 \\ 0.90 & \text { Federal Land Code 103 } & 0.90 \\ 0.90 & \text { Federal Land Code } 104 & 0.90 \\ 0.90 & \text { Federal Land Code } 105 & 0.90 \\ 0.90 & \text { Federal Land Code } 106 & 0.90 \\ 0.90 & \text { Federal Land Code } 107 & 0.90 \\ 0.90 & \text { Federal Land Code } 108 & 0.90 \\ 0.90 & \text { Federal Land Code } 198 & 0.90\end{array}$

Exist Prob

0.90 
R E S O U R C E D A T A B A S E I I S T I N G

DATE: $08 / 19 / 97$

PAGE NO: 32

FERC

Number

Plant Name

stream

State

NV00051 BOYD RESERVOIR

RABBIT CREEK

Name

County Name

River Basin

ELKO

HUMBOLDT RIVER BASIN

Class Owner Name

R F]RANK HOOPER

Name Plate

$\begin{array}{ll}\text { PESF } & \text { Annual Energy } \\ \text { Rating (MWh) }\end{array}$

PESF Annual

Rating ( $\mathrm{KW}$ )

$0.90 \quad 225.00$

913.00

Energy Rating

(MWh)

250.00

0.90

Unit Plant

Project

Dam

status

Latitude

Longitude 11532.00

821.70

Type

Type

status

wo

Factor

Wild/Scenic Protection

wild/Scenic Tributary or

Upstream/Downstream

wild/Scenic Location

Cultural value

Fish Presence Value

Geologic Value

Historic Value

other value

Recreation value

Scenic Value
Exist Prob

0.90

Wildlife value

Threatened/Endangered Fish

Threatened/Endangered Wildlife

0.90 Federal Land code 103

0.90 Federal Iand Code 104

0.90 Federal Land Code 105

0.90 Federal Land Code 106

0.90 Federal Land Code 107

0.90 Federal Land Code 108

0.90 Federal Land Code 198

0.90
Exist Prob

0.90

0.90

0.90

0.90

0.90

0.90

0.90

0.90

0.90

0.90 

R E S O U R C E
D A T A B A S E
I I S T I N G

DATE: $08 / 19 / 97$

PAGE NO: 33

FERC

Number

Plant Name

stream

State

Name

NV00055 ZUNINO RESERVOIR

SMITH CREEK

NV

County Name

River Basin

ELKO

HUMBOLDT RIVER BASIN

Class Owner Name

M ELKO COUNTY

Name Plate

Rating ( $\mathrm{KW}$ )

PESF

Annual Energy

Rating (MWh)

PESF Annual

107.00

$0.75 \quad 80.25$

406.00

Energy Rating (MWh)

Unit Plant

Project

Dam

Type

Type

status

status

Latitude

4028.00

c

DIV

XX

wo

Factor

Exist Prob

Factor

Exist Prob

wild/Scenic Protection

Wild/Scenic Tributary or

Upstream/Downstream

wild/Scenic Location

Cultural Value

Fish Presence Value

Geologic Value

Exist Prob

0.90

Wildlife Value

Threatened/Endangered Fish

304.50

Historic Value

Threatened/Endangered Wildlife

0.90 Federal Land Code 103

0.90 Federal Land Code 104

0.90 Federal Land Code 105

0.90 Federal Land Code 106

0.90 Federal Land code 107

other Value

Recreation Value

0.90

Federal Land Code 108

$\mathrm{Y} \quad \begin{aligned} & 0.75 \\ & 0.90\end{aligned}$

Federal Land Code 198

0.90

0.90

0.90

0.90

0.90

0.90

0.90

0.90

0.90

0.90 
RESOURC E D A TA B A S E I I S T I N G

DATE: $08 / 19 / 97$

PAGE NO: 34

FERC

Number

Plant Name

Stream

State

Name

NV00063 LOWER PITT-TAYLOR RES.

HUMBOLDT RIVER

NV

county Name

PERSHING
River Basin

HUMBOLDT RIVER BASIN

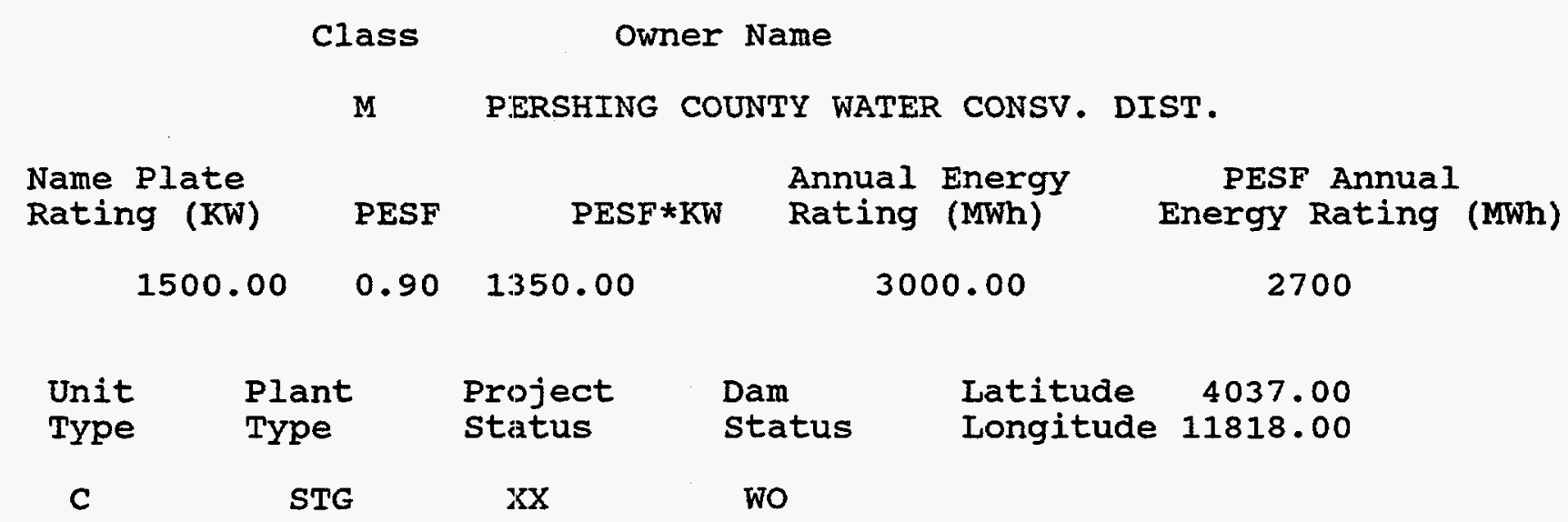

Factor

Wild/Scenic Protection

Wild/Scenic Tributary or Upstream/Downstream wild/Scenic Location cultural Value

Fish Presence Value

Geologic Value

Historic Value

other Value

Recreation Value

Scenic Value
Exist Prob

0.90

0.90

0.90

0.90

0.90

0.90

0.90

0.90

0.90
Factor

Exist Prob

Wildife value

Threatened/Endangered Fish

Threatened/Endangered Wildlife

Federal Iand Code 103

Federal Land Code 104

Federal Land Code 105

Federal Land Code 106

Federal Land Code 107

Federal Land Code 108

Federal Land Code 198
0.90

0.90

0.90

0.90

0.90

0.90

0.90

0.90

0.90

0.90 
DATE: $08 / 19 / 97$

FERC

Number

NV001

WATASHEAMU
Plant Name

County Name

DOUGLAS
PAGE NO: 35

State

Name

EAST FORK CARSON RIVER

NV
River Basin

CARSON RIVER BASIN

Class Owner Name

F BUREAU OF RECLAMATION

Name Plate Rating (KW) 8000.00 0.10800 .00

Unit Type

$$
\begin{gathered}
\text { Plant } \\
\text { Type } \\
\text { STG }
\end{gathered}
$$

C

Factor

Exist Prob
Annual Energy Rating (MWh) 34000.00
Latitude Longitude 11942.00

$\begin{array}{llr}\text { Dam } & \text { Latitude } & 3850.00 \\ \text { Status } & \text { Longitude } & 11942.00\end{array}$

$\mathrm{U}$
PESF Annual Energy Rating (MWh) 3400

Wild/Scenic Protection

wild/Scenic Tributary or Upstream/ Downstream wild/Scenic Location Cultural Value

Fish Presence Value

Geologic Value

Historic Value

other value

Recreation value

Scenic Value

Factor

$\mathrm{Y}$

\subsection{0}

\section{Wildlife Value} Threatened/Endangered Fish Threatened/Endangered Wildlife Exist Prob Federal Land code 103

0.90$$
\text { Federal Land Code } 104
$$

$Y$

$$
0.90
$$$$
\text { Federal Land Code } 105
$$$$
0.90 \text { Federal Land Code } 106
$$$$
0.90 \text { Federal Land Code } 107
$$$$
0.90 \text { Federal Land Code } 108
$$

Y 0.25 Federal Land Code 198

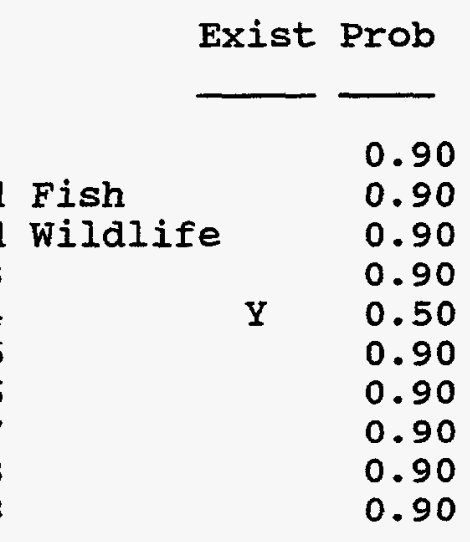



R E S O U R C E
D A T A B A S E
I I S T I N G

DATE: $08 / 19 / 97$

PAGE NO: 36

FERC

Number

Plant Name

Stream

State

NV00109 DAEK RESERVOIR

THOUSAND SPRINGS CREEK

Name

NVOO109 DAEK RESERVOIR

County Name

River Basin

ELKO

GREAT SALT LAKE BASIN

Class Owner Name

$R$ WALKER-WINECUP-GAMBLE INCORPORATED

Name Plate

Rating (KW)

PESF

Annual Energy

PESF Annual

1300.00

$0.90 \quad 1170.00$

2600.00

Energy Rating (MWh)

NV

\begin{tabular}{|c|c|c|c|c|c|}
\hline $\begin{array}{l}\text { Unit } \\
\text { Type }\end{array}$ & $\begin{array}{l}\text { Plant } \\
\text { Type }\end{array}$ & $\begin{array}{l}\text { Project } \\
\text { Status }\end{array}$ & $\begin{array}{l}\text { Dam } \\
\text { status }\end{array}$ & $\begin{array}{l}\text { Latitude } \\
\text { Longitude }\end{array}$ & $\begin{array}{r}4122.00 \\
11405.00\end{array}$ \\
\hline C & STG & $x x$ & wo & & \\
\hline
\end{tabular}

Factor

Exist Prob

Factor

Exist Prob

Wild/scenic Protection

Wild/Scenic Tributary or

0.90 Wildlife Value

Threatened/Endangered Fish

0.90

Threatened/Endangered Wildlife

0.90

Upstream/Downstream

wild/Scenic Location

Cultural value

Fish Presence Value

$\begin{array}{lll}0.90 & \text { Federal Land Code } 103 \\ 0.90 & \text { Federal Land code } 104\end{array}$

0.90

0.90 Federal Land Code 104

0.90

$\begin{array}{ll}0.90 & \text { Federal Land Code } 106 \\ 0.90 & \text { Federal Land Code } 107\end{array}$

0.90

Geologic Value

0.90

Historic Value

0.90 Federal Land Code 108

0.90

other Value

Recreation value

0.90 Federal Land Code 198

0.90

0.90

Scenic Value

0.90

0.90 
DATE: $08 / 19 / 97$

FERC

Number

Plant Name

NV00110 TWENTY THREE MILE RES.

County Name

ELKO
PAGE NO: 37

State

Name

NV
THOUSAND SPRINGS CREEK

River Basin

GREAT SALT LAKE BASIN

Class Owner Name

$R$ WALKER-WINECUP-GAMBLE INCORPORATED

Name Plate

Rating (KW)

1100.00

PESF

Annual Energy

PESF*KW Rating (MWh)

2300.00

$0.90 \quad 990.00$
Plant
Type
STG
Project Status
$\mathrm{XX}$
Dam
Status

Latitude $\quad 4132.00$

Type

C

Factor

Exist Prob

Factor

Exist Prob

Wild/Scenic Protection

wild/Scenic Tributary or

Upstream/Downstream

wild/Scenic Location

Cultural value

Fish Presence Value

Geologic Value

Historic Value

other Value

Recreation Value

Scenic Value
0.90 Wildife Value

Threatened/Endangered Fish

Threatened/Endangered Wildlife

0.90 Federal Land Code 103

0.90 Federal Land Code 104

0.90 Federal Land Code 105

0.90 Federal Land Code 106

0.90 Federal Land Code 107

0.90 Federal Land Code 108

0.90 Federal Land Code 198

0.90
PESF Annual

Energy Rating (MWh)

2070 wo 

R E S O U R C E
D A T A B A S E
L I S T I G

DATE: $08 / 19 / 97$

PAGE NO: 38

FERC

Number

Plant Name

stream

State

Name

NV00112 SILVER CREEK DAM

SILVER CREEK

NV

county Name

WHITE PINE
River Basin

GREAT SALT LAKE BASIN
Class Owner Name

R BAKER RANCHES, INCORPORATED

Name Plate

Rating ( $K W)$

PESF

$\mathrm{PESF} * \mathrm{KW}$

Annual Energy Rating (MWh)

1135.00

Latitude 3906.00

Unit

Plant

Type

Project status

C

STG
Dam
Status

$\mathrm{xx}$
PESF Annual Energy Rating (MWh)
Factor

Wild/Scenic Protection wild/Scenic Tributary or Upstream/Downstream wild/scenic Location Cultural value Fish Presence value Geologic Value Historic Value other Value Recreation value Scenic Value
Exist Prob

0.90

Wildlife Value Threatened/Endangered Fish Threatened/Endangered Wildife

0.90 Federal Land code 103

0.90 Federal Land Code 104

0.90 Federal Land Code 105

0.90 Federal Land Code 106

0.90 Federal Land Code 107

0.90 Federal Land Code 108

0.90 Federal Land Code 198

$$
1021.50
$$


DATE: $08 / 19 / 97$

FERC

Number

Plant Name

NV00113 BOWMAN RESERVOIR

County Name

CLARK

Class

$\mathbf{P}$

MUDDY RIVER IRRIGATION DISTRICT

Name Plate Rating (KW)

PESF

PESF $*$ KW

Annual Energy

Rating (MWh)

1018.00

$0.90 \quad 255.60$

284.00

Unit

Plant

Type

Project

status

$\mathrm{XX}$

Exist Prob

Factor $\begin{array}{llr}\text { Dam } & \text { Latitude } & 3637.00 \\ \text { Status } & \text { Longitude } & 11429.00\end{array}$

$\begin{array}{llr}\text { Dam } & \text { Latitude } & 3637.00 \\ \text { Status } & \text { Longitude } & 11429.00\end{array}$

wo
State

Name

NV
DIV$$
\text { C }
$$

Factor

Wild/Scenic Protection

wild/Scenic Tributary or Upstream/ Downstream wild/Scenic Location

Cultural value

Fish Presence Value

Geologic Value

Historic Value

other value

Recreation Value

Scenic Value
PESF Annual Energy Rating (MWh)

916.20
0.90
Wildlife value
Threatened/Endangered Fish
Threatened/Endangered Wildlife
0.90
0.90 Federal Land Code 103
0.90
0.90
Federal Land Code 104
0.90
0.90
Federal Land Code 105
0.90
0.90 Federal Land Code 106
0.90
0.90 Federal Land Code 107
0.90
0.90 Federal Land Code 108
0.90
Federal Land Code 198
0.90
0.90
0.90
0.90

Exist Prob 
R E S O U R C E D A T A B A S E I I S T I N G

DATE : $08 / 19 / 97$

PAGE NO: 40

FERC

Number

Plant Name

NV00127 GROVES LAKE

County Name

LANDER
Stream

KINGSTON CREEK

River Basin

BIG SMOKY BASIN

\section{Class Owner Name}

R NEVADA DEPARTMENT OF CONSERVATION

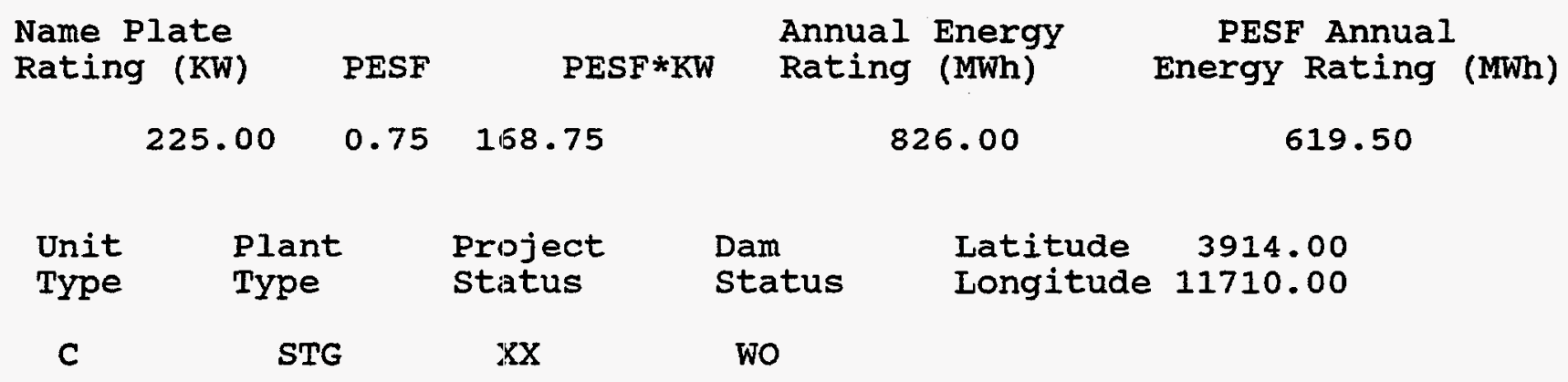

Factor

Wild/Scenic Protection

wild/Scenic Tributary or Upstream/Downstream wild/Scenic Location Cultural value

Fish Presence Value

Geologic Value

Historic Value

other Value

Recreation Value

Scenic Value
Exist Prob

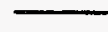

0.90

Wildlife value Threatened/Endangered Fish Threatened/Endangered Wildlife

0.90 Federal Land Code 103

0.90 Federal Land Code 104

0.90 Federal Land Code 105

0.90 Federal Land Code 106

0.90 Federal Land Code 107

0.90 Federal Iand Code 108

Y 0.75 Federal Land Code 198
Exist Prob

0.90

0.90

0.90

0.90

0.90

0.90

0.90

0.90

0.90

0.90 
DATE: $08 / 19 / 97$

FERC

Number

Plant Name

NV00174 COMINS LAKE

County Name

WHITE PINE

Class

Owner Name

M NEVADA DEPARTMENT OF TRANSPORTATION

Name Plate

Rating ( $\mathrm{KW})$

PESF

Annual Energy

PESF*KW

Rating (MWh)

381.00

Latitude $\quad 3910.00$

Unit

Plant

Type

Type

C

STG

Factor

Exist Prob

Factor

Exist Prob

Wild/Scenic Protection

Wild/Scenic Tributary or

Upstream/Downstream

wild/Scenic Location

Cultural value

Fish Presence Value

Geologic Value

Historic Value

other value

Recreation Value

Scenic Value
PAGE NO: 41

State

Name

NV
River Basin

STEPTOE BASIN
PESF Annual Energy Rating (MWh)
$0.75 \quad 76.50$

Project status

$\mathrm{Xx}$
Dam
status

wo

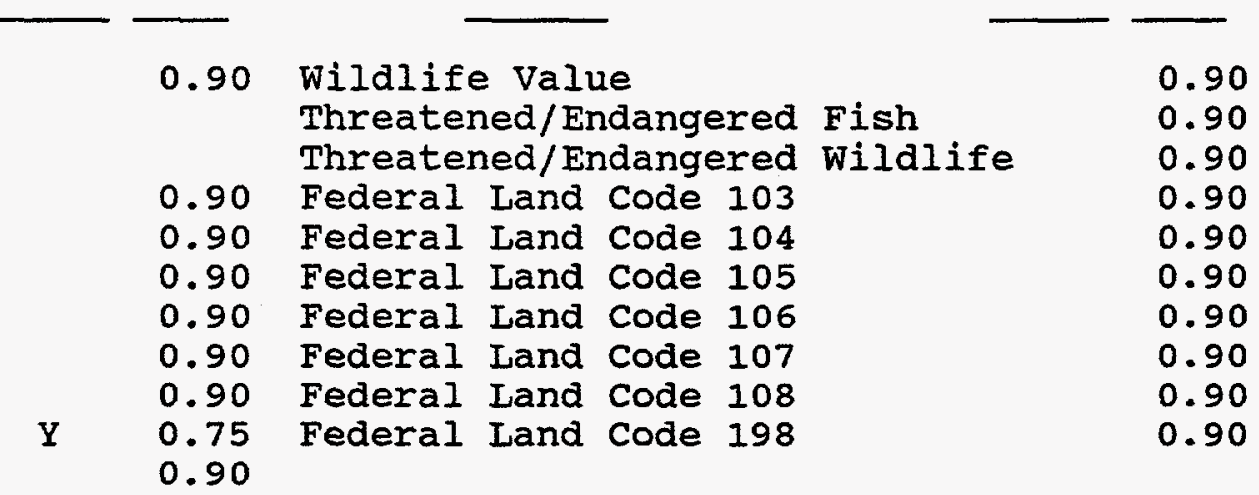



R E S O U R C E
D A T A B A S E
I I S T I N G

DATE: $08 / 19 / 97$

PAGE NO: 42

FERC

Number

Plant Name

Stream

State

NV00191 SEYLER RESERVOIR

PEAVINE CREEK

Name

NVO0191 SEYLER RESERVOIR

county Name

River Basin

NYE

BIG SMOKY BASIN

Class Owner Name

$R$ CARL HAAS

Name Plate

Rating (KW)

PESF

Annual Energy

PESF*KW Rating (MWh)

PESF Annual

$$
\begin{array}{lll}
53.00 & 0.90 \quad 47.70
\end{array}
$$

207.00

Energy Rating (MWh)

$\begin{array}{lllll}\text { Unit } & \text { Plant } & \text { Project } & \text { Dam } & \text { Latitude } 3832.00 \\ \text { Type } & \text { Type } & \text { Stitus } & \text { Status } & \text { Longitude 11714.00 }\end{array}$

C

STG

$\mathrm{XX}$

Wo

Factor

wild/Scenic Protection

Wild/Scenic Tributary or Upstream/Downstream wild/scenic Location Cultural value Fish Presence Value Geologic Value Historic Value other Value Recreation value Scenic Value
Exist Prob

0.90

Wildlife Value Threatened/Endangered Fish Threatened/Endangered Wildlife

0.90 Federal Land Code 103

0.90 Federal Land Code 104

0.90 Federal Land Code 105

0.90 Federal Land code 106

0.90 Federal Land Code 107

0.90 Federal Land code 108

0.90 Federal Land Code 198

NV

186.30

0.90

0.90

0.90

0.90

0.90

0.90

0.90

0.90

0.90

0.90

0.90 
FERC

Number

NV00197 SOLDIER MEADOWS RESERVOIR SOLDIER CREEK

River Basin

HUMBOLDT

BLACK ROCK DESERT BASIN

State Name

NV

\section{Class Owner Name \\ R SUSAN VALLEY RANCH}

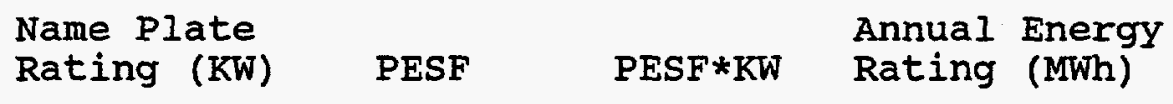

$116.00 \quad 0.90 \quad 104.40$

Unit Plant Project

Type

Type

C

STG

\begin{abstract}
status
\end{abstract}
$\mathrm{xx}$

$$
452.00
$$

Latitude $\quad 4124.00$

Dam

status

$$
\text { Longitude } 11909.00
$$

Factor

Wild/Scenic Protection

Wild/scenic Tributary or Upstream/Downstream wild/Scenic Location Cultural value

Fish Presence Value

Geologic Value

Historic value

other Value

Recreation Value

Scenic Value

Exist Prob

Factor

Exist Prob

0.90 Wildife Value

Threatened/Endangered $F$ ish

Threatened/Endangered Wildlife

0.90 Federal Land Code 103

0.90 Federal Iand Code 104

0.90 Federal Land Code 105

0.90 Federal Land Code 106

0.90 Federal Land Code 107

0.90 Federal Land Code 108

0.90 Federal Land Code 198

0.90
PESF Annual Energy Rating (MWh)

406.80 
DATE: $08 / 19 / 97$

PAGE NO: 44

FERC

Number

Plant Name

stream

State

NV00199 INCLINE IAKE

THIRD CREEK

Name

NV

county Name

WASHOE
River Basin

IAAKE TAHOE BASIN

\section{Class Owner Name}

R IINCLINE LAAKE CORPORATION

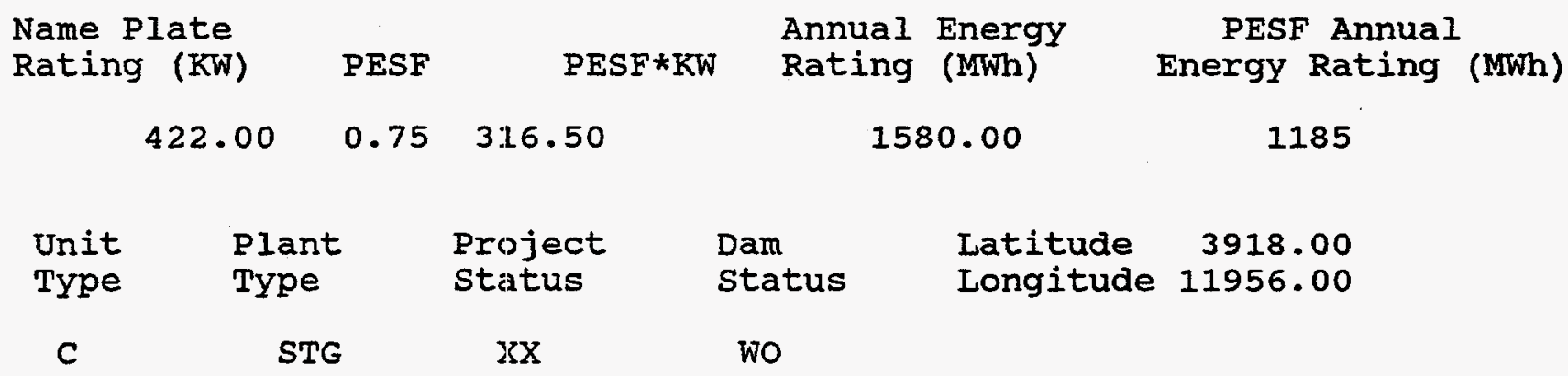

Factor

Wild/Scenic Protection

wild/Scenic Tributary or Upstream/Downstream wild/Scenic Location Cultural Value

Fish Presence Value

Geologic Value

Historic Value

other value

Recreation value

Scenic Value
Exist Prob

0.90

Wildlife value

Threatened/Endangered Fish

Threatened/Endangered Wildlife

0.90 Federal Land code 103

0.90 Federal Land code 104

0.90 Federal Land code 105

0.90 Federal Land code 106

0.90 Federal Land code 107

0.90 Federal Land Code 108

$\mathrm{Y} \quad 0.75$ Federal Land Code 198
Exist Prob

0.90

0.90

0.90

0.90

0.90

0.90

0.90

0.90

0.90

0.90 
DATE: $08 / 19 / 97$

FERC

Number

Plant Name

NV00226 SOUTH FORK RESERVOIR

County Name

ELKO

Class

Owner Name

M NEVADA DEPARTMENT OF CONSERVATION

Name Plate

Rating (KW)

PESF

PESF*KW

Annual Energy

Rating (MWh)

4900.00

Latitude $\quad 4041.00$

Unit

Plant

$0.75 \quad 2175.00$

Type

Project Status

Dam

Type

STG

$\mathrm{xx}$

Exist Prob

Factor

Exist Prob

Factor

Wild/Scenic Protection

wild/Scenic Tributary or Upstream/Downstream wild/Scenic Location

Cultural value

Fish Presence Value

Geologic Value

Historic value

other Value

Recreation Value

Scenic Value
PAGE NO: 45

State

Name

NV
PESF Annual Energy Rating (MWh)

3675

$\begin{array}{clll}0.90 & \begin{array}{l}\text { Wildife Value } \\ \text { Threatened/Endangered Fish }\end{array} & 0.90 \\ & & 0.90 \\ 0.90 & \text { Threatened/Endangered Wildlife } & 0.90 \\ 0.90 & \text { Federal Land Code 103 } & 0.90 \\ 0.90 & \text { Federal Land Code 104 } & 0.90 \\ 0.90 & \text { Federal Land Code 105 } & 0.90 \\ 0.90 & \text { Federal Land Code 107 } & 0.90 \\ 0.90 & \text { Federal Land Code 108 } & 0.90 \\ 0.75 & \text { Federal Land Code 198 } & 0.90 \\ & & 0.90\end{array}$


DATE: $08 / 19 / 97$

FERC

Number

Plant Name

NVO0234 EAST PEAK LAKE

county Name

DOUGLAS

\section{class}

R HEAVENLY VALLEY RESORT

Name Plate

Rating (KW)

PESF

$\mathrm{PESF} * \mathrm{KW}$

Annual Energy

131.00

$0.90 \quad 117.90$

473.00

PAGE NO: 46
River Basin

CARSON RIVER BASIN state

Name

NV

$\begin{array}{cccllr}\text { Unit } & \text { Plant } & \text { Project } & \text { Dam } & \text { Latitude } & 3856.00 \\ \text { Type } & \text { Type } & \text { Status } & \text { Status } & \text { Longitude } 11954.00 \\ \text { C } & \text { STG } & \text { XX } & \text { WO } & \end{array}$

Factor

Wild/Scenic Protection

wild/Scenic Tributary or Upstream/ Downstream wild/Scenic Location Cultural Value Fish Presence value Geologic Value Historic Value other Value Recreation Value Scenic Value
Exist Prob

Factor

Exist Prob

0.90 Wildlife Value Threatened/Endangered Fish Threatened/Endangered Wildlife

0.90 Federal Land Code 103

0.90 Federal Land Code 104

0.90 Federal Land Code 105

0.90 Federal Land Code 106

0.90 Federal Land Code 107

0.90 Federal Land Code 108

0.90 Federal Land Code 198
0.90

0.90

0.90

0.90

0.90

0.90

0.90

0.90

0.90

0.90
PESF Annual Energy Rating (MWh)

425.70

0.90 

R E S O U R E
D A T A B A S E
L I S T N G

DATE: $08 / 19 / 97$

PAGE NO: 47

FERC

Number

Plant Name

Stream

State

Name

NV00536 ROUND MOUNTAIN HYDRAULIC

JEFFERSON \& SHOSHONE CREEKS

NV

County Name

River Basin

NYE

BIG SMOKY BASIN

$\begin{array}{cc}\text { Class } & \text { Owner Name } \\ R & \text { ROUND MOUNTAIN HYDRAULIC MINING } C O .\end{array}$

Name Plate

Annual Energy

Rating ( $K W$ ) PESF PESF*KW Rating (MWh)

PESF Annual

$82.00 \quad 0.90 \quad 73.80$

324.00

Energy Rating (MWh)

$0.90 \quad 73.80$

Unit Plant Project

Type

Type

status

Dam

status

Latitude $\quad 3844.00$

C

DIV

$\mathrm{xx}$

$\mathrm{U}$

Factor

Exist Prob

Factor

Exist Prob

wild/Scenic Protection

Wild/Scenic Tributary or

Upstream/Downstream wild/scenic Location Cultural Value

Fish Presence Value

Geologic Value

Historic Value

other Value

Recreation Value

Scenic Value

0.90 Wildlife Value

Threatened/Endangered Fish Threatened/Endangered Wildlife

0.90 Federal Land Code 103

0.90 Federal Land Code 104

0.90 Federal Land Code 105

0.90 Federal Land Code 106

0.90 Federal Land code 107

0.90 Federal Land Code 108

0.90 Federal Land Code 198

0.90
291.60 

R ESOU R C E
D A T A B A S E
L I S T I G

DATE: $08 / 19 / 97$

PAGE NO: 48

FERC

Number

Plant Name

NV01151 CHIMNEY RESERVOIR

county Name

HUMBOLDT

Class

R NEVADA GARVEY RANCHES

Name Plate

Rating (KW)
95.00

PESF

$0.75 \quad 71.25$

\section{Plant}

Type

Unit

C

STG
Annual Energy Rating (MWh)
State

Name

NV
River Basin

HUMBOLDT RIVER BASIN
Factor

Wild/Scenic Protection wild/Scenic Tributary or Upstream/Downstream wild/Scenic Location Cultural value Fish Presence Value Geologic Value Historic Value other Value Recreation Value Scenic Value
Exist Prob

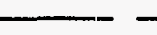

PESF Annual Energy Rating (MWh)

341.00

255.75

$\begin{array}{llr}\text { Dam } & \text { Latitude } & 4124.00 \\ \text { Status } & \text { Longitude } & 11711.00 \\ \text { wo } & \end{array}$

Factor

Exist Prob

0.90 Wildlife Value

Threatened/Endangered Fish

Threatened/Endangered Wildlife

0.90

0.90

0.90

0.90 Federal Iand Code 103

0.90 Federal Land Code 104

0.90 Federal Land Code 105

0.90

0.90

0.90

0.90

0.90

0.90

0.90 

R E S O U R C E
D A T A B A S E
L I S T I N G

DATE: $08 / 19 / 97$

PAGE NO: 49

FERC

Number

NV01198 FLORISTON MILI

County Name

WASHOE
Plant Name

Class

$\mathbf{R}$

FLORISTON PULP AND PAPER COMPANY

Name Plate

Rating (KW)

427.00

PESF

Annual Energy

PESF*KW

$0.90 \quad 384.30$

Plant

Type

Type

ROR

C

Factor

Exist Prob

0.90

Wildife Value

Threatened/Endangered Fish

Wild/Scenic Tributary or

Upstream/Downstream

wild/Scenic Location

Cultural Value

Fish Presence Value

Geologic Value

Historic Value

other Value

Recreation Value

Scenic Value
BRONCO AND DRY PRARIE CREEKS

River Basin

TRUCKEE RIVER BASIN $\begin{array}{llr}\text { Dam } & \text { Latitude } & 3927.00 \\ \text { Status } & \text { Longitude } & 12000.00\end{array}$

wo
0.90 Federal Land Code 104

0.90 Federal Land Code 105

0.90 Federal Land Code 106

0.90 Federal Land Code 107

0.90 Federal Land Code 108

0.90 Federal Land Code 198

0.90
0.90 Federal Land code 103 state

Name

NV
PESF Annual

Energy Rating (MWh)

$$
1621.00
$$

1458.90
Exist Prob

0.90

0.90

0.90

0.90

0.90

0.90

0.90

0.90

0.90

0.90 
R E S O U R C E D A T A B A S E I I S T I N G

DATE : $08 / 19 / 97$

PAGE NO: 50

FERC

Number

Plant Name

Stream

State

NV01412 OPHIR POWER PLANT

OPHIR CREEK

Name

\section{MV1412 OPHIR POWER PLANT}

County Name

WASHOE

River Basin

TRUCKEE RIVER BASIN

$\begin{array}{cr}\text { Class } & \text { Owner Name } \\ \text { R } & \text { DOUGLAss, W. G. }\end{array}$

Name Plate

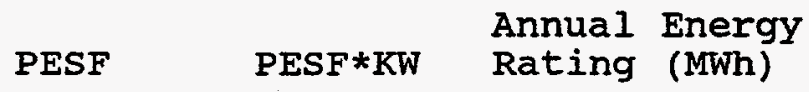

PESF Annual

Rating ( $\mathrm{KW})$

$0.75 \quad 251.25$

1263.00

NV

$$
335.00
$$

0.75

Project status

$\begin{array}{ll}\text { Unit } & \text { Plant } \\ \text { Type } & \text { Type }\end{array}$

C

ROR

$\mathrm{X} X$

Dam
status

Latitude

Longitude 11952.00
Factor

Wild/Scenic Protection

Wild/Scenic Tributary or Upstream/Downstream wild/Scenic Location Cultural value Fish Presence Value Geologic Value Historic Value other Value Recreation value Scenic value
Exist Prob

$\begin{array}{cc} & \\ & 0.90 \\ & \\ & 0.90 \\ & 0.90 \\ & 0.90 \\ & 0.90 \\ & 0.90 \\ Y & 0.75 \\ & 0.90 \\ Y & 0.90\end{array}$

Factor

Exist Prob

Wildlife Value Threatened/Endangered Fish Threatened/Endangered Wildlife

0.90

0.90

0.90

Federal Land Code 103

Federal Land Code 104

Federal Land Code 105

Federal Land Code 106

Federal Land Code 107

Federal Land Code 108

Federal Land Code 198
0.90

0.90

0.90

0.90

0.90

0.90

0.90 
DATE : $08 / 19 / 97$

FERC

Number

Plant Name

NV03720 CURLEY-WOOD

County Name

ELKO
PAGE NO: 51

JARBIDGE RIVER

state

stream

Name

NV

River Basin

SNAKE RIVER BASIN

Class Owner Name

R T. J. CURLEY AND W. T. WOOD

Name Plate

Rating ( $K W$ )

PESF

Annual Energy

PESF*KW

Rating (MWh)

PESF Annual

305.00

$0.50 \quad 152.50$

1094.00

547

\begin{tabular}{|c|c|c|c|c|}
\hline $\begin{array}{l}\text { Unit } \\
\text { Type }\end{array}$ & $\begin{array}{l}\text { Plant } \\
\text { Type }\end{array}$ & $\begin{array}{l}\text { Project } \\
\text { status }\end{array}$ & $\begin{array}{l}\text { Dam } \\
\text { status }\end{array}$ & $\begin{array}{lr}\text { Latitude } & 4152.00 \\
\text { Longitude } & 11526.00\end{array}$ \\
\hline C & ROR & $X X$ & W & \\
\hline
\end{tabular}

Factor

Exist Prob

Factor

Exist Prob

Wild/Scenic Protection

wild/Scenic Tributary or

Upstream/Downstream

wild/Scenic Location

Cultural Value

Fish Presence Value

Geologic Value

Historic Value

other Value

Recreation Value

Scenic Value

$\begin{array}{ccll} & 0.90 & \begin{array}{l}\text { Wildife Value } \\ \text { Threatened/Endangered Fish }\end{array} & 0.90 \\ & & \text { Threatened/Endangered Wildlife } & 0.90 \\ & 0.90 & \text { Federal Land Code 103 } & 0.90 \\ 0.90 & \text { Federal Land Code 104 } & 0.90 \\ 0.90 & \text { Federal Land Code 105 } & 0.90 \\ & 0.90 & \text { Federal Land Code 106 } & 0.90 \\ & 0.75 & \text { Federal Land Code 107 } & 0.90 \\ \text { Y } & 0.90 & \text { Federal Land Code 108 } & 0.90 \\ & 0.75 & \text { Federal Land Code 198 } & 0.90 \\ \text { Y } & 0.90 & & 0.90\end{array}$



R E S O U R C E
D A T A B A S E
L I S T IN $\mathrm{G}$

DATE: $08 / 19 / 97$

PAGE NO: 52

FERC

Number

Plant Name

Stream

State

NV03898 ROUND MOUNTAIN

JETT CREEK

Name

County Name

River Basin

NYE

BIG SMOKY BASIN

Class Owner Name

R ROUND MOUNTAIN MINING COMPANY

Name Plate

$\begin{array}{lll} & \text { Annual Energy } \\ \text { PESF } & \text { PESF*KW } & \text { Rating (MWh) }\end{array}$

PESF Annual

Rating (KW)

$0.90 \quad 73.80$

324.00

Energy Rating

(MWh)

82.00

$0.90 \quad 7.3 .80$

Unit Plant

Project status

Dam

status

Latitude

Longitude 11714.00

291.60

C

DIV

XX

Wo

Factor

Exist: Prob

Wild/Scenic Protection

Wild/Scenic Tributary or Upstream/ Downstream wild/Scenic Location Cultural Value

Fish Presence Value

Geologic Value

Historic Value

other Value

Recreation Value

Scenic Value

Existe Prob
0.90 Wildlife Value
Threatened/Endan

Threatened/Endangered Fish

Threatened/Endangered Wildlife

0.90 Federal Land Code 103

0.90 Federal Land Code 104

0.90 Federal Land Code 105

0.90 Federal Land Code 106

0.90 Federal Land Code 107

0.90 Federal Land Code 108

0.90 Federal Land Code 198
Exist Prob

0.90

0.90

0.90

0.90

0.90

0.90

0.90

0.90

0.90

0.90 
R E S O U R C E

DATE : $08 / 19 / 97$
D A T A B A S E

L I S T I N G

PAGE NO: 53

FERC

Number

Plant Name

NV06850 BOWERS MANSION PLANT

county Name

WASHOE

River Basin

TRUCKEE RIVER BASIN

FRANKTOWN CREEK

state

Name

NV

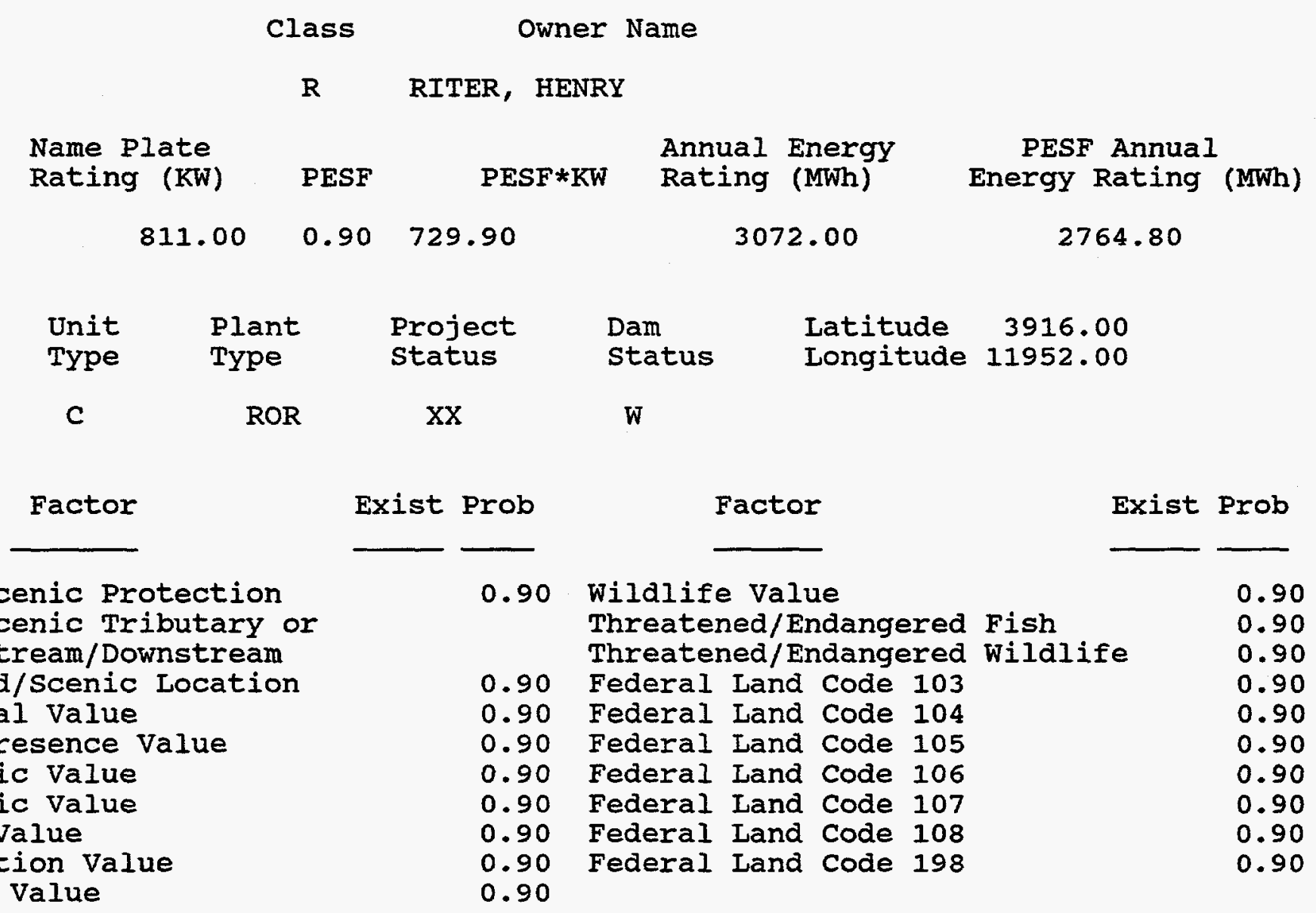

Wild/Scenic Protection

wild/Scenic Tributary or

Upstream/Downstream wild/Scenic Location

Cultural value

Fish Presence Value

Geologic Value

Historic Value

other value

Recreation Value

Scenic Value

Exist Prob

0.90

Wildife value

Threatened/Endangered Fish

0.90

Federal Land Code 103

Federal Land Code 104

0.90 Federal Land Code 105

0.90 Federal Land Code 106

0.90 Federal Land Code 107

0.90 Federal Land Code 108

0.90

Federal Land Code 198
0.90

0.90

0.90

0.90

0.90

0.90

0.90

0.90

0.90 
DATE: $08 / 19 / 97$

FERC

Number

Plant Name

NV07847 PIERMONT MILL

county Name

WHITE PINE
PAGE NO: 54

State

Name

NV

$\begin{array}{cc}\text { Class } & \text { Owner Name } \\ \text { R } & \text { ELY CALUMET MINING CORPORATION }\end{array}$

Name Plate

Rating (KW)

PESF

Annual Energy

PESF*KW

Rating (MWh)

PESF Annual

85.00

$0.90 \quad 76.50$

338.00

304.20

$\begin{array}{cccll}\text { Unit } & \text { Plant } & \text { Project } & \text { Dam } & \text { Latitude } 3929.00 \\ \text { Type } & \text { Type } & \text { Status } & \text { Status } & \text { Longitude } 11433.00 \\ \text { C } & \text { ROR } & X X & U & \end{array}$

Factor

Exist Prob

Factor

Exist Prob

Wild/Scenic Protection

Wild/Scenic Tributary or

0.90 Wildlife Value

Threatened/Endangered Fish

Upstream/Downstream Threatened/Endangered Wildlife

0.90

wild/Scenic Location

0.90 Federal Land Code 103

0.90

0.90 Federal Land Code 104

0.90

Cultural value

Fish Presence Value

0.90 Federal Land Code 105

0.90

0.90 Federal Land Code 106

0.90

Geologic Value

Historic Value

0.90 Federal Land Code 107

0.90

other Value

0.90 Federal Land Code 108

0.90

Recreation Value

0.90 Federal Land Code 198

0.90

Scenic Value

0.90

0.90 
RESOUR C E

DATE: $08 / 19 / 97$
D A T A B A S E

FERC

Number

NV09344 PECETTI

County Name

WASHOE

Class

$\mathbf{R}$

CASAZZA AND PECETTI

Name Plate Rating (KW)

River Basin

THOMAS CREEK
Annual Energy

PESF*KW

TRUCKEE RIVER BASIN

PAGE NO: 55

I S T I N G

State

Name

NV

$$
\begin{array}{lll}
142.00 & 0.90 \quad 127.80
\end{array}
$$

502.00

PESF Annual Energy Rating (MWh)

451.80

$\begin{array}{cccllr}\text { Unit } & \text { Plant } & \text { Project } & \text { Dam } & \text { Latitude } & 3923.00 \\ \text { Type } & \text { Type } & \text { Status } & \text { Status } & \text { Longitude } 11947.00 \\ \text { C } & \text { ROR } & \text { XX } & W & \end{array}$

Factor

Exist Prob

Factor

Exist Prob

Wild/Scenic Protection

Wild/Scenic Tributary or

0.90 Wildlife Value

Threatened/Endangered Fish

Upstream/Downstream

wild/Scenic Location

Cultural value

Fish Presence Value

Threatened/Endangered Wildlife

0.90

0.90

0.90 Federal Land code 103

0.90

0.90 Federal Land code 104

0.90

0.90 Federal Land code 105

0.90

0.90 Federal Land Code 106

0.90

0.90 Federal Land Code 107

0.90

Historic value

0.90 Federal Land Code 108

0.90

other Value

Recreation value

0.90 Federal Land Code 198

0.90

0.90

0.90 

R E S O U R C E
D A T A B A S E
I I S T I N G

DATE: $08 / 19 / 97$

PAGE NO: 56

FERC

Number

Plant Name

stream

State

NV10101 CAT CREEK RESERVOIR

CAT CREEK

Name

NV

County Name

River Basin

LYON

WALKER IAKE BASIN

\section{Class Owner Name}

F U.S. ARMY

Name Plate

Rating ( $K W$ )

PESF

Annual Energy

PESF*KW

Rating (MWh)

PESF Annual

630.00

$0.50 \quad 3.15 .00$

2397.00

1198.50

Energy Rating (MWh)

\begin{tabular}{|c|c|c|c|c|}
\hline $\begin{array}{l}\text { Unit } \\
\text { Type }\end{array}$ & $\begin{array}{l}\text { Plant } \\
\text { Type }\end{array}$ & $\begin{array}{l}\text { Project } \\
\text { status }\end{array}$ & $\begin{array}{l}\text { Dam } \\
\text { status }\end{array}$ & $\begin{array}{lr}\text { Latitude } & 3833.00 \\
\text { Longitude } 11843.00\end{array}$ \\
\hline C & STG & $x x$ & wo & \\
\hline
\end{tabular}

Factor

Wild/Scenic Protection

Wild/Scenic Tributary or

Upstream/Downstream

wild/Scenic Location

Cultural value

Fish Presence value

Geologic Value

Historic Value

other Value

Recreation value

Scenic Value
Exist Prob

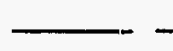

Exist Prob

Factor

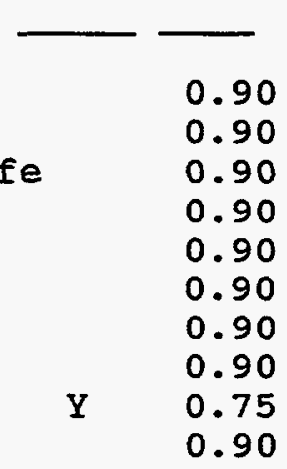


DATE: $08 / 19 / 97$

PAGE NO: 57

FERC

Number

NV10114 PALISADES \#2

County Name

EUREKA
Plant Name

County Name
River Basin

HUMBOLDT RIVER BASIN
State

Name

PINE CREEK NV

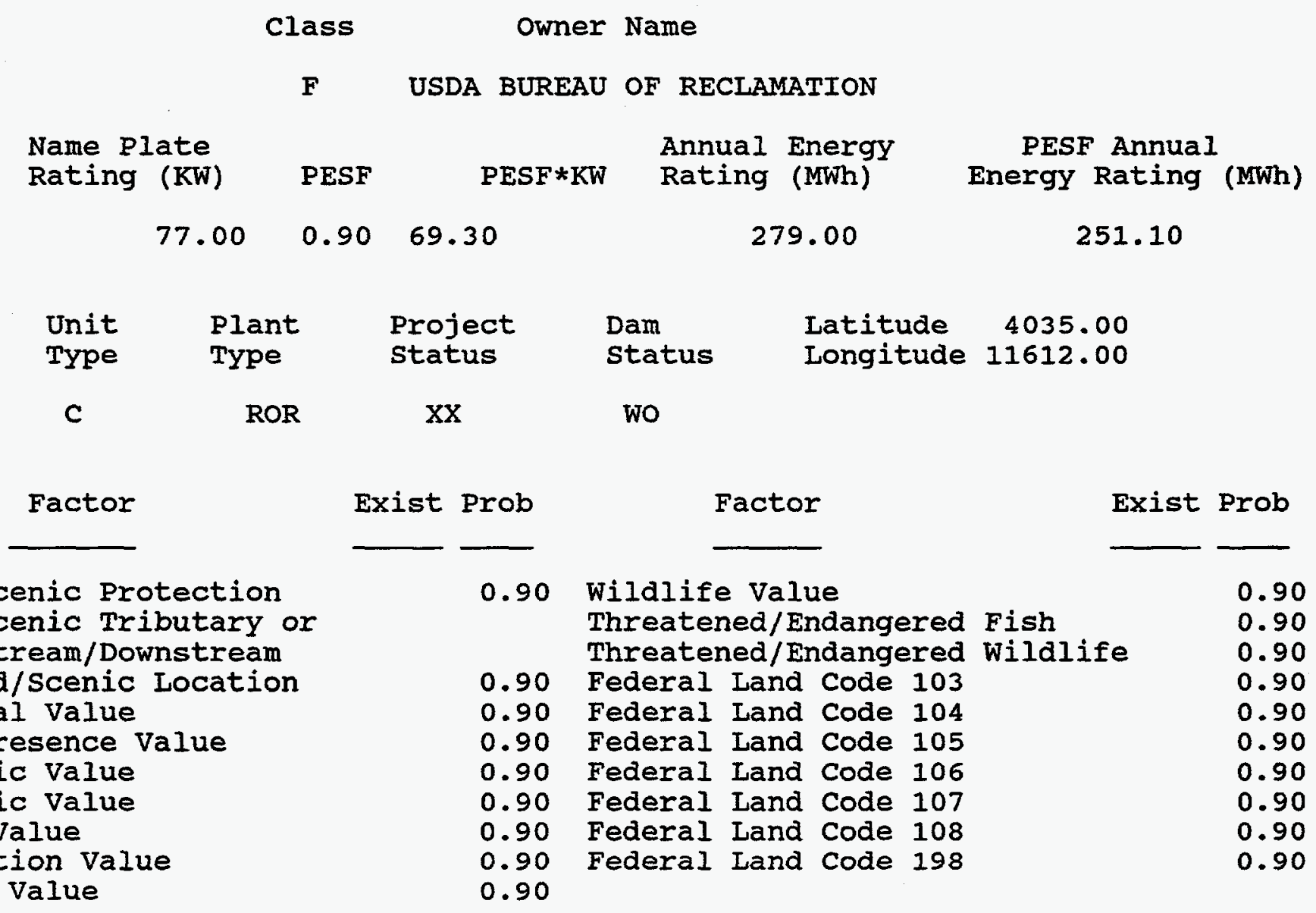

Wild/Scenic Protection

Wild/Scenic Tributary or Upstream/Downstream wild/Scenic Location Cultural value Fish Presence Value Geologic Value Historic Value other Value Recreation value Scenic Value

0.90
0.90

$$
\text { Annual Energy }
$$$$
279.00
$$$$
\text { Latitude } \quad 4035.00
$$$$
\text { Longitude } 11612.00
$$

0.90

0.90

0.90

0.90

0.90

0.90

0.90

0.90 
DATE: $08 / 19 / 97$

PAGE NO: 58

FERC

Number

Plant Name

Stream

State

NV10120 CARSON RIVER DIVERSION

CARSON RIVER

Name

NV

County Name

River Basin

CHURCHILL

CARSON RIVER BASIN

Class Owner Name

P TIRUCKEE-CARSON IRRIGATION DISTRICT

Name Plate

Rating (KW)

PESF

Annual Energy

2200.00

$0.75 \quad 1550.00$

4300.00

PESF Annual

Energy Rating (MWh)

3225

$\begin{array}{ccccc}\text { Unit } & \text { Plant } & \text { Project } & \text { Dam } & \text { Latitude } \\ \text { Type } & \text { Type } & \text { Status } & \text { Status } & \text { Longitude } 11859.00 \\ \text { C } & \text { ROR } & \text { XX } & \text { WO } & \end{array}$

Factor

Exist Prob

Factor

Exist Prob

Wild/Scenic Protection

Wild/Scenic Tributary or

Upstream/Downstream

wild/Scenic Location

Cultural Value

Fish Presence Value

Exist

0.90

Wildife Value

Threatened/Endangered Fish

Threatened/Endangered Wildlife

0.90

0.90 Federal Land Code 103

0.90

0.90 Federal Land Code 104

0.90

0.90 Federal Land Code 105

0.90

0.90 Federal Land Code 106

0.90

Geologic Value

Historic Value

other Value

Recreation Value

0.90 Federal Land code 107

0.90

$\mathrm{Y}$

0.75 Federal Land Code 108

0.90

0.90 Federal Land Code 198

0.90

Scenic Value

0.90

0.90 
DATE: $08 / 19 / 97$

PAGE NO: 59

FERC

Number

Plant Name

stream

state

Name

NV10121 DERBY DIVERSION DAM

TRUCKEE RIVER

NV

county Name

River Basin

STOREY

TRUCKEE RIVER BASIN

$\begin{array}{cc}\text { Class } & \text { Owner Name } \\ \text { F } & \text { USDI BUREAU OF RECLAMATION }\end{array}$

Name Plate

Rating (KW)

Annual Energy PESF PESF*KW Rating (MWh)

PESF Annual $\begin{array}{cc} & 2300.00 \\ \text { Unit } & \text { Plant } \\ \text { Type } & \text { Type } \\ \text { C } & \text { ROR }\end{array}$ $0.50 \quad 1150.00$ 3200.00 Energy Rating (MWh)

Project status

$\mathrm{xx}$

Exist Prob

Factor

Wild/Scenic Protection

Wild/Scenic Tributary or Upstream/Downstream wild/Scenic Location

cultural value

Fish Presence Value

Geologic Value

Historic Value

other Value

Recreation value

Scenic Value $\begin{array}{llr}\text { Dam } & \text { Latitude } & 3935.00 \\ \text { Status } & \text { Longitude } & 11927.00\end{array}$

wo
1600 
FERC

Number

Plant Name

NV10132 WEBER DAM

County Name

MINERAL

Class

$\mathbf{F}$

Name Plate

Rating (KW)

1700.00

0.75

Plant

Type

C

STG

Factor

Wild/Scenic Protection

wild/Scenic Tributary or

Upstream/Downstream

wild/Scenic Location

Cultural value

Fish Presence Value

Geologic Value

Historic Value

other value

Recreation Value

Scenic Value
1275.00

Project

status

Exist: Prob

0.90

Wildlife Value

Threatened/Endangered Fish

Threatened/Endangered Wildlife

Annual Energy

2900.00

Iatitude 3902.00

Longitude 11806.00

\section{5}

wo
State Name

NV
PESF Annual Energy Rating (MWh)
0.90 Federal Land Code 103

0.90 Federal Land Code 104

0.90 Federal Land Code 105

0.90 Federal Land Code 106

0.90 Federal Land Code 107

0.90 Federal Land Code 108

0.90 Federal Land Code 198
Exist Prob

0.90

0.90

0.90

0.90

0.90

0.90

0.90

$Y$

0.75

0.90

0.90 
DATE : $08 / 19 / 97$

FERC

Number

NV10133 STILLWATER POINT

county Name

CHURCHILL
PAGE NO: 61

State

Name

CARSON RIVER

NV

River Basin

CARSON RIVER BASIN

$\begin{array}{cc}\text { Class } & \text { Owner Name } \\ \text { F } & \text { USDI FISH AND WILDLIFE SERVICE }\end{array}$

Name Plate

Annual Energy

Rating (KW)

PESF

Rating (MWh)

PESF Annual

1100.00

$0.10 \quad 110.00$

1900.00

190

\begin{tabular}{|c|c|c|c|c|}
\hline $\begin{array}{l}\text { Unit } \\
\text { Type }\end{array}$ & $\begin{array}{l}\text { Plant } \\
\text { Type }\end{array}$ & $\begin{array}{l}\text { Project } \\
\text { status }\end{array}$ & $\begin{array}{l}\text { Dam } \\
\text { Status }\end{array}$ & $\begin{array}{lr}\text { Latitude } & 3932.00 \\
\text { Longitude } & 11829.00\end{array}$ \\
\hline C & DIV & $x x$ & wo & \\
\hline
\end{tabular}

Factor

Exist Prob

Factor

Exist Prob

wild/Scenic Protection

Wild/Scenic Tributary or

Upstream/Downstream wild/Scenic Location

Cultural Value

Fish Presence Value

Geologic Value

Historic Value

other Value

Recreation value

Scenic Value

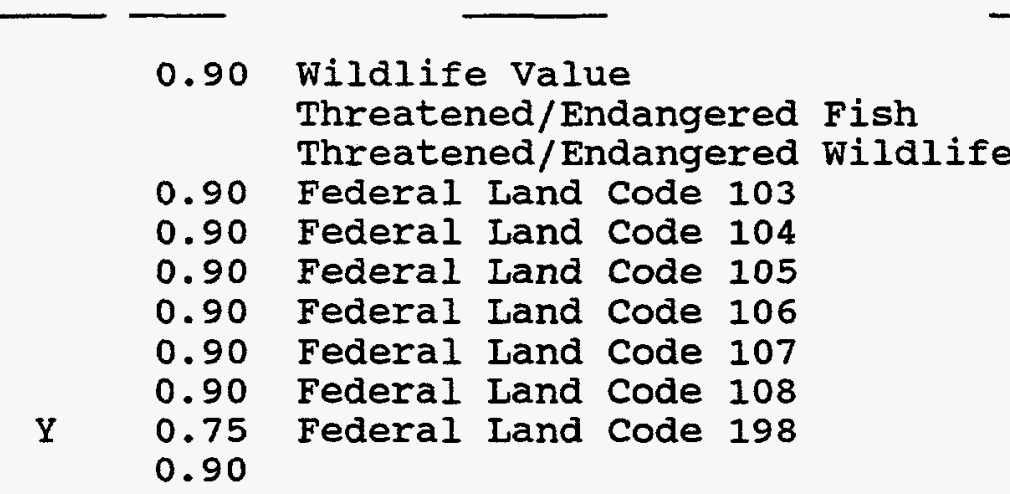

0.75

0.90

0.90

0.90

0.90

Y

0.10

0.90

0.90

0.90

0.90 


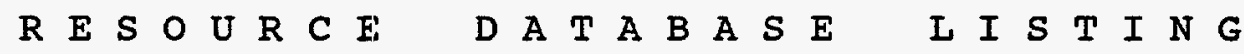

DATE: $08 / 19 / 97$

PAGE NO: 62

FERC

Number

Plant Name

stream

State

NV10170 LOGAN CREEK

LOGAN CREEK

Name

county Name

DOUGILAS

River Basin

LAKE TAHOE BASIN

$\begin{array}{cc}\text { Class } & \text { Owner Name } \\ \text { R } & \text { LCIGAN CREEK ESTATES }\end{array}$

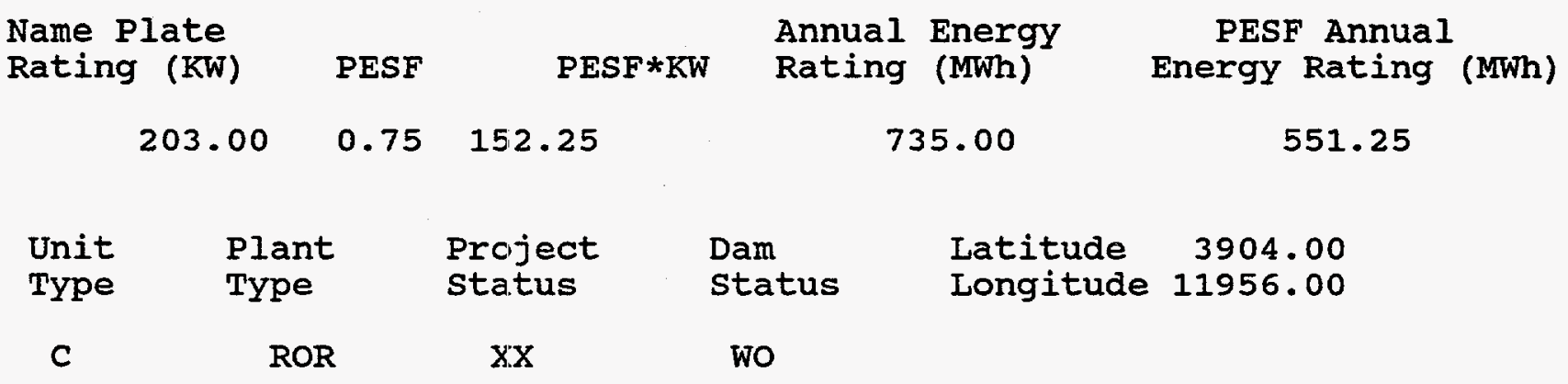

Factor

Wild/scenic Protection

Wild/Scenic Tributary or Upstream/Downstream wild/Scenic Location Cultural value

Fish Presence Value Geologic Value Historic Value other value Recreation Value Scenic Value
Exist Prob

0.90 Wildlife Value Threatened/Endangered Fish Threatened/Endangered Wildiife

Federal Land Code 103 Federal Land code 104 Federal Land code 105 Federal Land Code 106 Federal Land Code 107 Federal Land Code 108 Federal Land code 198

0.90

0.90
Exist Prob

0.90

0.90

0.90

0.90

0.90

0.90

0.90

0.90

0.90

0.90

0.90 

R E S O U R C E
D A T A B A S E
I I S I N G

DATE : $08 / 19 / 97$

PAGE NO: 63

FERC

Number

Plant Name

stream

State

Name

NV10189 HEADGATE DAM

SALMON FAILLS CREEK

NV

county Name

River Basin

ELKO

SNAKE RIVER BASIN

$\begin{array}{cr}\text { Class } & \text { Owner Name } \\ \text { R } & \text { EYER BOIES }\end{array}$

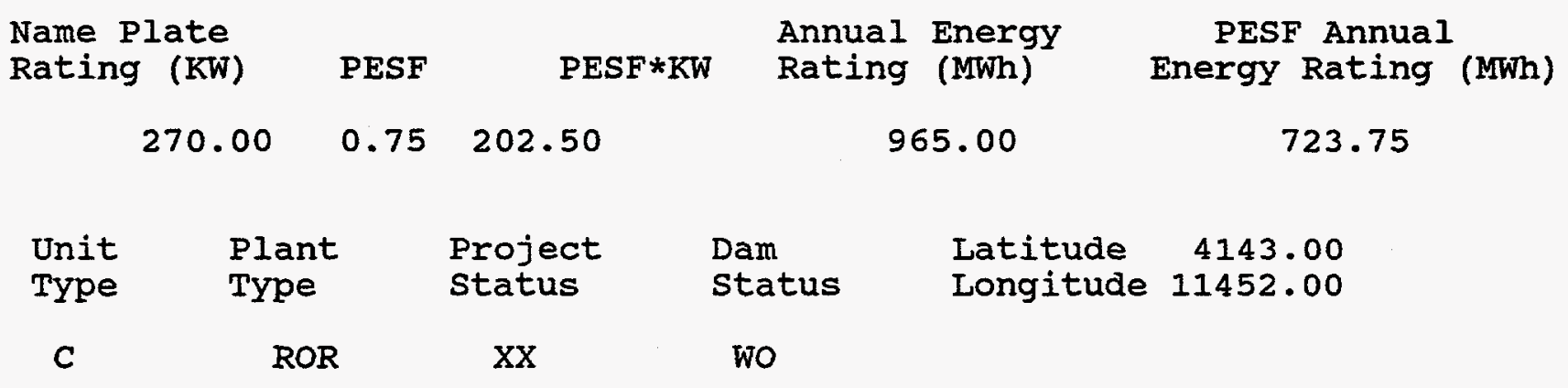

Factor

Exist Prob

Factor

Exist Prob

Wild/scenic Protection

Wild/Scenic Tributary or

Upstream/Downstream wild/Scenic Location

Cultural Value

Fish Presence Value

Geologic Value

Exist Prob

-

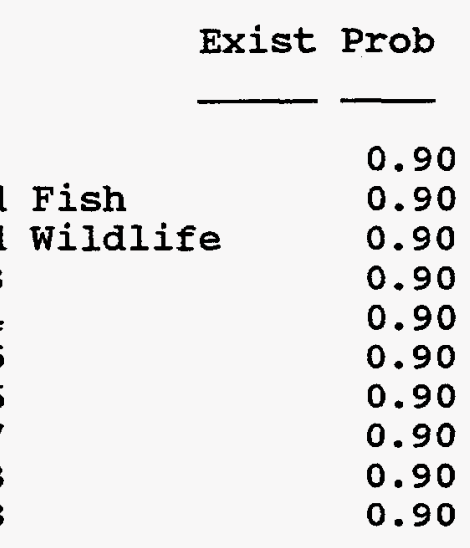

0.90 Wildlife value 0.90

Threatened/Endangered Fish 0.90

Threatened/Endangered Wildlife 0.90

0.90 Federal Land Code $103 \quad 0.90$

0.90 Federal Land Code $104 \quad 0.90$

$Y \quad 0.75$ Federal Land Code $105 \quad 0.90$

0.90 Federal Land Code $106 \quad 0.90$

0.90 Federal Land Code $107 \quad 0.90$

0.90 Federal Land Code $108 \quad 0.90$

$\begin{array}{llll}\text { Recreation Value } & 0.90 & \text { Federal Land Code } 198 & 0.90 \\ \text { Scenic Value } & 0.90 & & \end{array}$ 
R E S O U R C E D A T A B A S E I I S T I N G

DATE: $08 / 19 / 97$

PAGE NO: 64

FERC

Number

Plant Name

stream

State

NV10222 B-B MINE RESERVOIR

ROCK CREEK

Name

NV

county Name

River Basin

ESMERALDA

FISH LAKE BASIN

class Owner Name

R PAUL KOHLSMAN

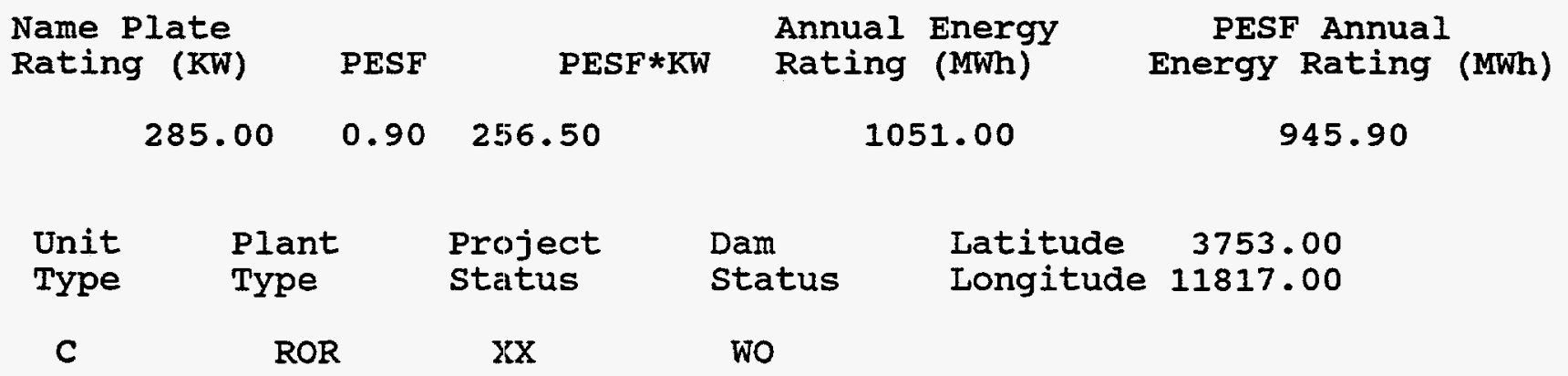

Factor

Wild/Scenic Protection

wild/Scenic Tributary or Upstream/Downstream

wild/Scenic Iocation

Cultural Value

Fish Presence Value

Geologic Value

Historic Value

other Value

Recreation Value

Scenic Value
Exist: Prob

0.90

Wildlife Value

Threatened/Endangered Fish

Threatened/Endangered Wildlife

0.90 Federal Land Code 103

0.90 Federal Land Code 104

0.90 Federal Land Code 105

0.90 Federal Land Code 106

0.90 Federal Land Code 107

0.90 Federal Land Code 108

0.90 Federal Land Code 198

0.90

Exist Prob

0.90

0.90

0.90

0.90

0.90

0.90

0.90

0.90

0.90

0.90 
$\begin{array}{llllllll}R & E & S & O & U & R & C\end{array}$

DATE: $08 / 19 / 97$
D A T A B A $\mathrm{S} E$

I I S T I N G

PAGE NO: 65

FERC

Number

Plant Name

Stream

state

Name

NV10223 CHIATOVICH CREEK RESERVOIR CHIATOVICH CREEK

NV

\author{
County Name \\ ESMERALDA \\ River Basin \\ FISH LAKE BASIN
}

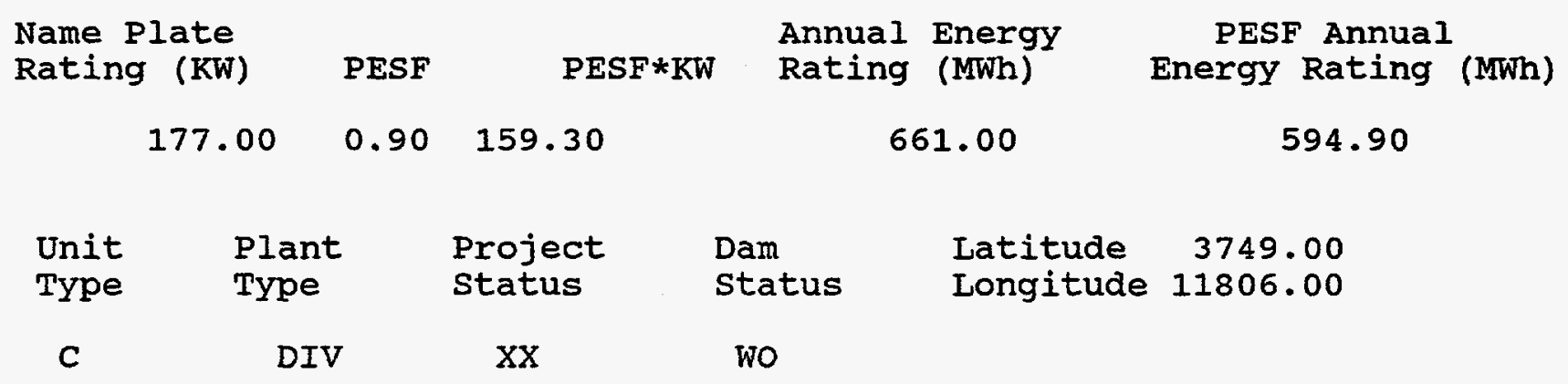

Factor

Wild/Scenic Protection

wild/Scenic Tributary or Upstream/Downstream wild/Scenic Location Cultural value Fish Presence Value Geologic Value Historic Value other Value

Recreation Value

Scenic Value
Exist Prob

0.90

Wildlife value Threatened/Endangered Fish Threatened/Endangered Wildlife

0.90 Federal Land Code 103

0.90 Federal Land Code 104

0.90 Federal Land Code 105

0.90 Federal Land Code 106

0.90 Federal Land Code 107

0.90 Federal Land Code 108

0.90 Federal Land Code 198
Exist Prob

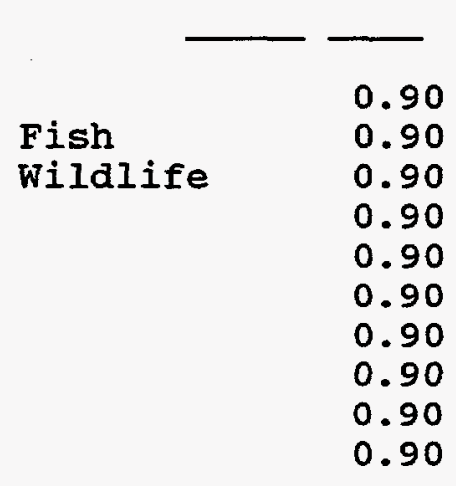


RE SOURCE D A T A B A S E L I S T I G

DATE : $08 / 19 / 97$

PAGE NO: 66

FERC

Number

Plant Name

Stream

state

NV10322 RATTLESNAKE RESERVOIR

QUEEN CREEK

Name

WVIO322 RATILESNAKE RESERVOIR

County Name

River Basin

MINERAL

OWENS RIVER BASIN

Class Owner Name

R QUENN VALLEY RANCH

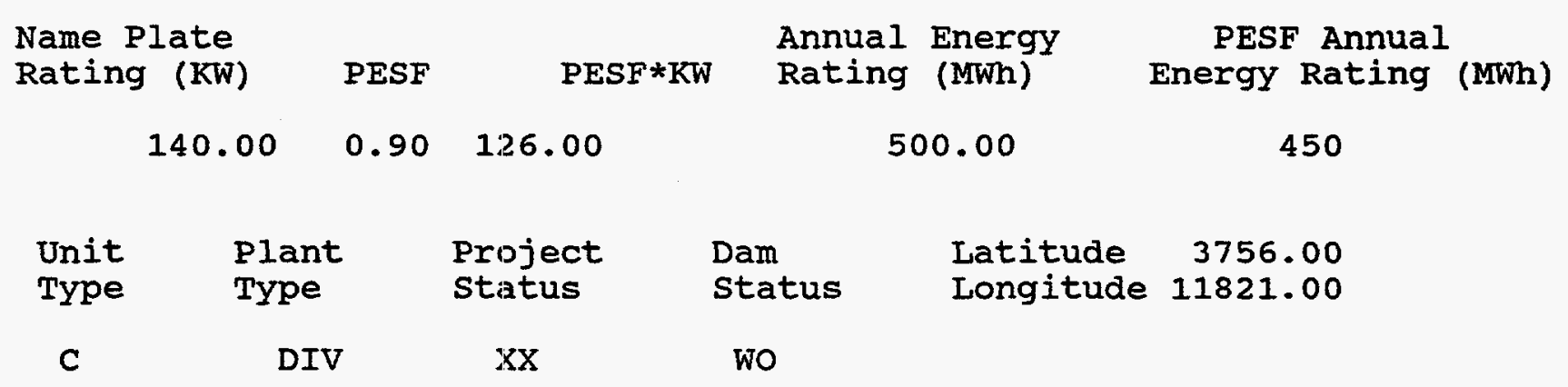

Factor

Exist Prob

Factor

Exist Prob

Wild/Scenic Protection

wild/Scenic Tributary or

0.90 Wildlife Value

Threatened/Endangered Fish

NV

Upstream/Downstream

Wild/Scenic Location

Cultural value

Fish Presence Value

Geologic Value

Historic Value

other Value

Recreation Value

Scenic Value Threatened/Endangered Wildlife

0.90

0.90

0.90

0.90 Federal Land Code 103

0.90

0.90 Federal Land code 104

0.90

0.90 Federal Land code 105

0.90

0.90 Federal Land Code 106

0.90

0.90 Federal Land Code 107

0.90

0.90 Federal Land Code 108

0.90

0.90 Federal Land Code 198

0.90 

R E S O U R C E
D A T A B A S E
I I S T I N G

DATE: $08 / 19 / 97$

PAGE NO: 67

FERC

Number

Plant Name

Stream

State

Name

NV10460 CALLAGHAN CREER RESERVOIR CALLAGHAN CREEK

NV

county Name

LANDER
River Basin

GRASS BASIN

$$
\begin{array}{cr}
\text { Class } & \text { Owner Name } \\
\text { R } & \text { MOLLY F. KNUDTSEN }
\end{array}
$$

Name Plate Rating ( $K W$ )

83.00

PESF

$0.90 \quad 74.70$

Unit

Type

$$
\begin{aligned}
& \text { Plant } \\
& \text { TYpe } \\
& \text { STG }
\end{aligned}
$$

C

Factor

Exist Prob

Wild/Scenic Protection

wild/Scenic Tributary or Upstream/Downstream wild/Scenic Location Cultural value

Fish Presence Value

Geologic Value

Historic Value

other Value

Recreation Value

Scenic Value
Annual Energy

PESF*KW Rating (MWh)

316.00

Latitude $\quad 3940.00$

\section{Longitude 11655.00 \\ Status}

PESF Annual

Energy Rating (MWh)

284.40

wo

0.90

Factor

Exist Prob

\section{Wildlife Value}

Threatened/Endangered Fish

Threatened/Endangered Wildlife

0.90 Federal Land Code 103

0.90 Federal Land Code 104

0.90 Federal Land Code 105

0.90 Federal Land Code 106

0.90 Federal Land Code 107

0.90 Federal Land Code 108

0.90 Federal Iand code 198

0.90
0.90

0.90

0.90

0.90

0.90

0.90

0.90

0.90

0.90

0.90 
DATE: $08 / 19 / 97$

PAGE NO: 68

FERC

Number

Plant Name

Stream

State

NV10470 TOPAZ RESERVOIR

WAIKER RIVER

Name

NV

county Name

DOUGLAS
River Basin

WALKER IAKE BASIN

\section{class Owner Name}

P WAIKER RIVER IRRIGATION DISTRICT

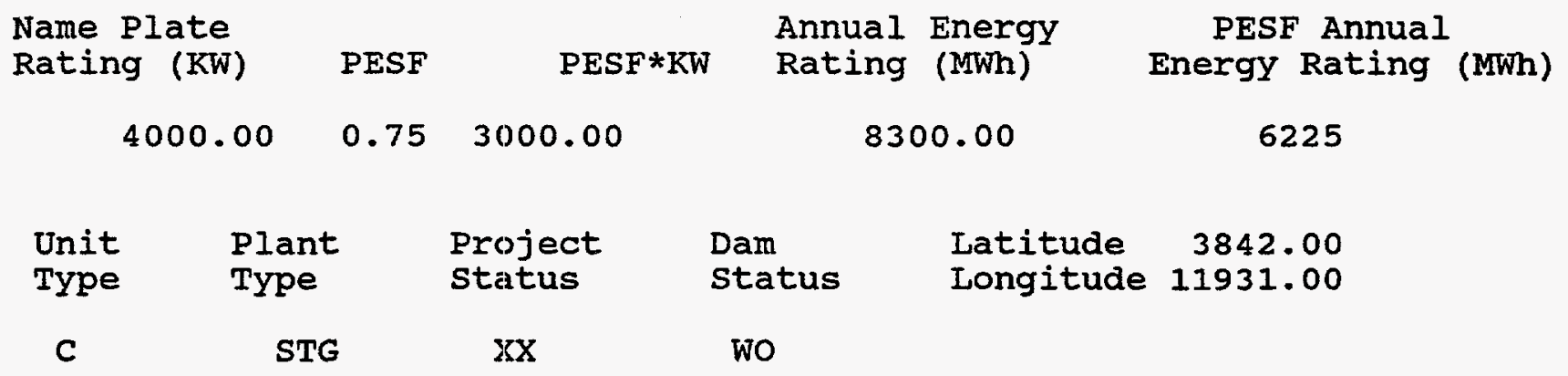

Factor

Wild/Scenic Protection

Wild/Scenic Tributary or Upstream/Downstream

wild/Scenic Location

Cultural Value

Fish Presence Value

Geologic Value

Historic Value

other Value

Recreation Value

Scenic Value
Exist: Prob

0.90

Wildlife Value

Threatened/Endangered Fish

Threatened/Endangered Wildiife

0.90 Federal Land Code 103

0.90 Federal Land Code 104

0.90 Federal Land Code 105

0.90 Federal Land Code 106

0.90 Federal Land Code 107

0.90 Federal Land Code 108

$Y \quad 0.75$

0.90

Federal Land Code 198
Exist Prob

0.90

0.90

0.90

0.90

0.90

0.90

0.90

0.90

0.90

0.90 
DATE : 08/19/97

FERC

Number

Plant Name

NV10472 WASHOE LAKE

county Name

WASHOE

class

Owner Name

$\mathbf{R}$

BRIAN

B. WALTERS
Name Plate Rating ( $\mathrm{KW}$ )
PESF
Annual Energy

PESF*KW
Rating (MWh)

261.00 state

Name

NV

$$
69.00 \quad 0.75 \quad 51.75
$$

$\begin{array}{cccc}\text { Unit } & \begin{array}{c}\text { Plant } \\ \text { Type }\end{array} & \begin{array}{l}\text { Project } \\ \text { Status }\end{array} & \begin{array}{l}\text { Dam } \\ \text { Status }\end{array} \\ \text { C } & \text { STG } & \text { XX } & \text { wo }\end{array}$

Factor

Exist Prob

Exist Prob

Wild/Scenic Protection

wild/Scenic Tributary or Upstream/Downstream wild/Scenic Location

Cultural value

Fish Presence Value

Geologic Value

Historic Value

other Value

Recreation Value

Scenic Value

Factor

Exist Prob

0.90

Wildlife Value

Threatened/Endangered Fish

Threatened/Endangered Wildlife

0.90 Federal Land Code 103

0.90 Federal Land Code 104

0.90 Federal Land Code 105

0.90 Federal Land Code 106

0.90 Federal Land Code 107

0.90 Federal Land Code 108

$\mathrm{Y} \quad 0.75$ Federal Land Code 198
PESF Annual Energy Rating (MWh)

195.75 
DATE: $08 / 19 / 97$

PAGE NO: 70

FERC

Number

Plant Name

NV20004 REDHEAD DAM

county Name

DOUGLAS
River Basin

CARSON RIVER BASIN
State

Name

NV

$$
\begin{array}{cc}
\text { Class } & \text { Owner Name } \\
\text { F } & \text { U.S.D.I. BUREAU OF RECLAMATION }
\end{array}
$$

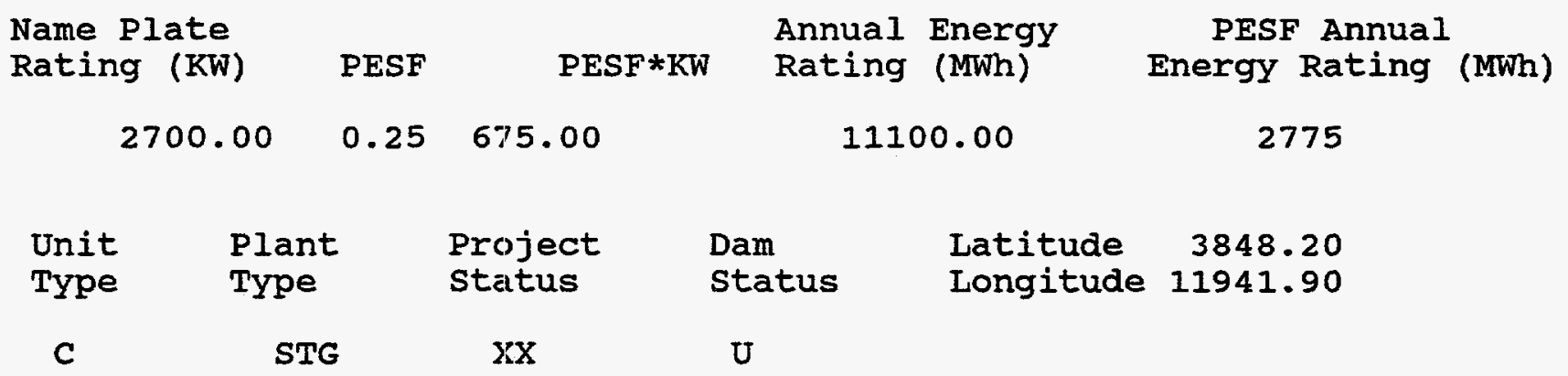

\section{Factor}

Wild/Scenic Protection wild/scenic Tributary or Upstream/Downstream wild/Scenic Location Cultural value Fish Presence Value Geologic Value Historic Value other Value Recreation value Scenic Value
Exist: Prob

Factor

0.90 Wildlife Value Threatened/Endangered Fish Threatened/Endangered Wildlife

0.90 Federal Land code 103

0.90 Federal Land Code 104

0.90 Federal Land Code 105

0.90 Federal Land Code 106

0.90 Federal Land Code 107

0.90 Federal Land Code 108

$\mathrm{Y} \quad 0.25$

Federal Land Code 198
Exist Prob

0.90

0.90

0.90

0.90

0.90

0.90

0.90

0.90

0.90

0.90 
DATE: $08 / 19 / 97$

FERC

Number

Plant Name

NV2U001 LAWTON DAM

county Name

WASHOE
PAGE NO: 71

State

Name

TRUCKEE RIVER

NV

River Basin

TRUCKEE RIVER BASIN

Class Owner Name

C UNKNOWN

Name Plate

Rating ( $K W$ )

4100.00
PESF

$0.10 \quad 410.00$
Annual Energy

Rating (MWh)

19200.00
PESF Annual

Energy Rating (MWh)

$\begin{array}{cc}\text { Unit } & \text { Plant } \\ \text { Type } & \text { Type } \\ \text { C } & \text { STG }\end{array}$

Factor

Wild/Scenic Protection

Wild/Scenic Tributary or Upstream/Downstream wild/Scenic Location Cultural Value

Fish Presence Value

Geologic Value

Historic Value

other value

Recreation value

Scenic Value

Project
Status

$\mathrm{XX}$

$\begin{array}{llr}\text { Dam } & \text { Latitude } & 3930.70 \\ \text { Status } & \text { Longitude } & 11954.70\end{array}$

$\mathrm{U}$
Exist Prob

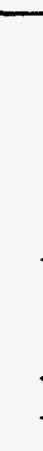

$\begin{array}{llll} & 0.90 & \text { Wildlife Value } & 0.90 \\ & & \text { Threatened/Endangered Fish } & 0.90 \\ & & \text { Threatened/Endangered Wildlife } & 0.90 \\ & 0.90 & \text { Federal Land Code 103 } & 0.90 \\ & 0.90 & \text { Federal Land Code } 104 & 0.90 \\ \text { Y } & 0.25 & \text { Federal Land Code 105 } & 0.90 \\ & 0.90 & \text { Federal Land Code 106 } & 0.90 \\ & 0.90 & \text { Federal Land Code } 107 & 0.90 \\ \text { Y } & 0.50 & \text { Federal Land Code } 108 & 0.90 \\ Y & 0.25 & \text { Federal Land Code 198 } & 0.90\end{array}$

Factor

Exist Prob

0.90

0.90

0.90

0.90

0.90

0.90

0.90 
RE SOUR C E D A T A B A S E L I S T I N G

DATE: $08 / 19 / 97$

PAGE NO: 72

FERC

Number

Plant Name

NV2U002 VERDI DAM

county Name

River Basin

WASHOE

TRUCKEE RIVER BASIN

Class Owner Name

C UNKNOWN

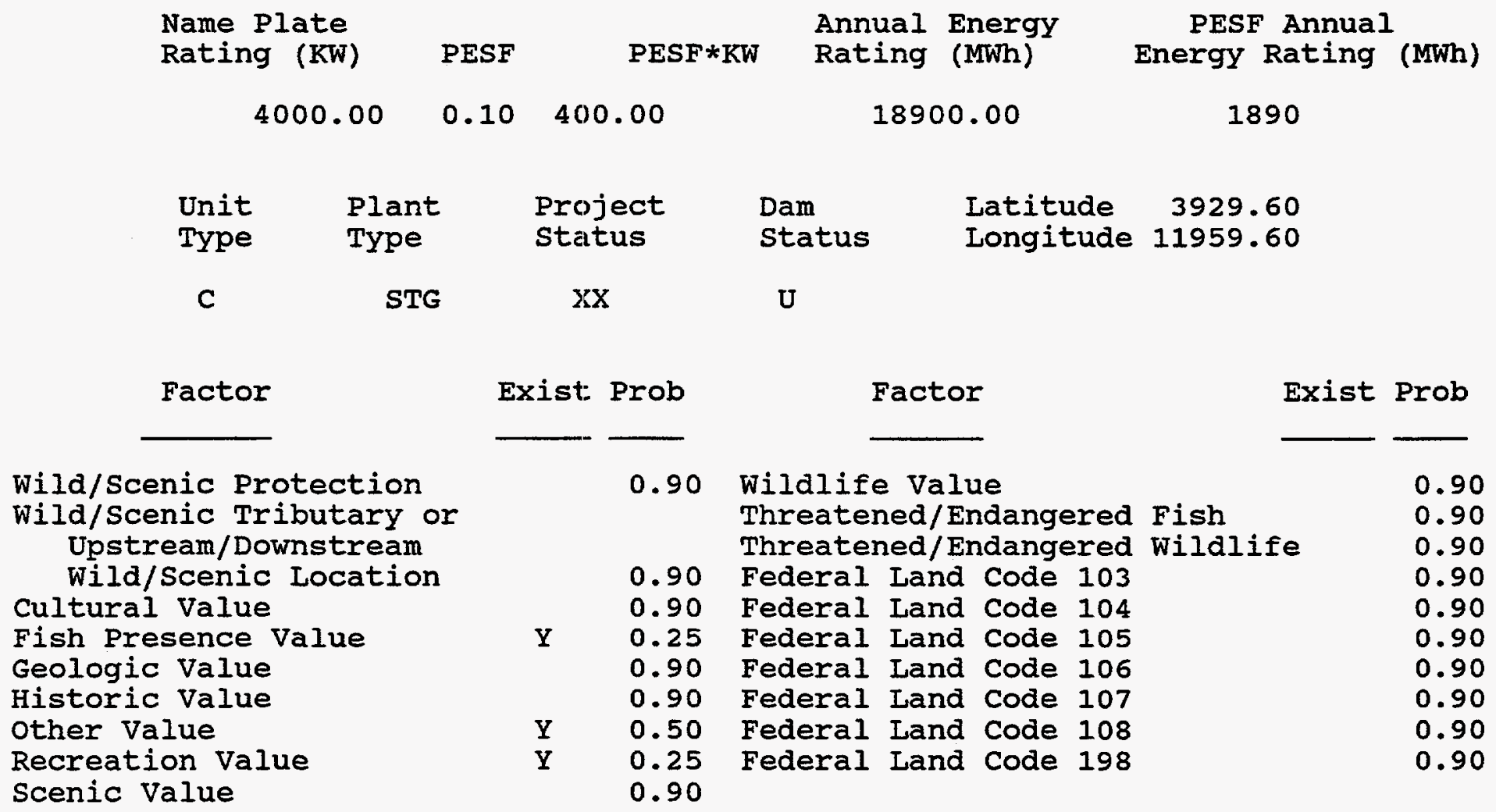


DATE: $08 / 19 / 97$

FERC

Number

Plant Name

NV2U003 MEXICAN DAM

County Name

CARSON CITY

Class Owner Name

C UNKNOWN

Name Plate

Rating ( $\mathrm{KW}$ )

PESF

Annual Energy

3500.00

$0.50 \quad 1750.00$

PESF*KW

Rating (MWh)

14300.00

PAGE NO: 73

CARSON RIVER

River Basin

CARSON RIVER BASIN
State

Name

NV

$\begin{array}{ccccc}\text { Unit } & \text { Plant } & \text { Project } & \text { Dam } & \text { Latitude } \\ \text { Type } & \text { Type } & \text { Status } & \text { Status } & \text { Longitude } 11939.70 \\ \text { C } & \text { ROR } & \mathrm{XX} & \mathrm{U} & \end{array}$

Factor

Wild/Scenic Protection

wild/Scenic Tributary or

Upstream/Downstream wild/Scenic Location

Cultural value

Fish Presence Value

Geologic Value

Historic Value

other Value

Recreation Value

Scenic Value
Exist Prob

Factor

Exist Prob

0.90 Wildlife Value

Threatened/Endangered Fish

Threatened/Endangered Wildlife

0.90 Federal Land Code 103

0.90 Federal Land Code 104

0.90 Federal Land Code 105

0.90 Federal Land Code 106

0.90 Federal Land Code 107

$\mathrm{Y}$

0.50 Federal Land Code 108

0.90 Federal Land Code 198

0.90
PESF Annual

Energy Rating (MWh)

7150 
FERC

Number

Plant Name

NV2U005 PEKOE DAM

County Name

WASHOE

\section{Class Owner Name}

C UNKNOWN

Name Plate

Rating (KW)

PESF

PESF*KW

Annual Energy

Rating (MWh)

8200.00

Latitude $\quad 3946.10$

$\begin{array}{ll}\text { Unit } & \text { Plant } \\ \text { Type } & \text { Type }\end{array}$

Project status

Dam

status

Longitude 11920.20

820

STG

XX

U

Factor

Wild/Scenic Protection Wild/Scenic Tributary or Upstream/ Downstream wild/Scenic Location Cultural value Fish Presence Value Geologic Value Historic Value other Value Recreation value Scenic Value
Exist Prob

Factor

Exist Prob

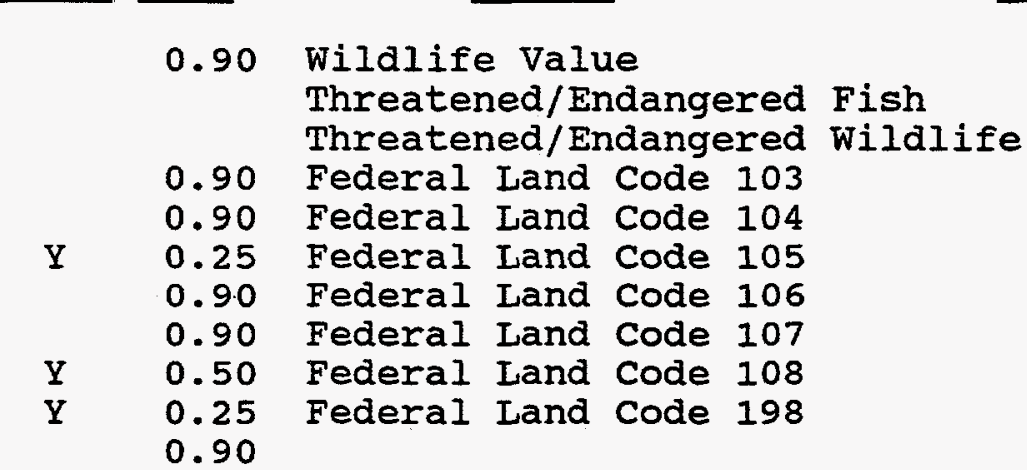

state Name

NV
PESF Annual Energy Rating (MWh) 
DATE: $08 / 19 / 97$

FERC

Number

Plant Name

NV2UC06 HUDSON RESERVOIR

County Name

LYON

Class Owner Name

C UNKNOWN

Name Plate

Rating (KW)

PESF

$\mathrm{PESF} * \mathrm{KW}$

Annual Energy Rating (NWh)

stream

State

Name

WEST WALKER RIVER

NV

PESF Annual

Energy Rating (MWh)
1400.00
0.50700 .00
6300.00

$\begin{array}{ccccc}\text { Unit } & \text { Plant } & \begin{array}{l}\text { Project } \\ \text { Status }\end{array} & \begin{array}{l}\text { Dam } \\ \text { Status }\end{array} & \begin{array}{l}\text { Latitude } \\ \text { Longitude }\end{array} \\ \text { Type } & \text { Typ48.70 } & \\ \text { C } & \text { STG } & \mathrm{XX} & \mathrm{U} & \end{array}$

Factor

Exist Prob

Factor

Wild/Scenic Protection

wild/Scenic Tributary or

Upstream/Downstream

wild/Scenic Location

Cultural Value

Fish Presence Value

Geologic Value

Historic Value

other Value

Recreation Value

Scenic Value

0.90 Wildlife Value

Threatened/Endangered Fish

Threatened/Endangered Wildlife

0.90 Federal Land code 103

0.90 Federal Land Code 104

0.90 Federal Iand Code 105

0.90 Federal Land Code 106

0.90 Federal Land Code 107

Y $\quad 0.50$ Federal Land Code 108

0.90 Federal Land Code 198

0.90
Exist Prob

3150

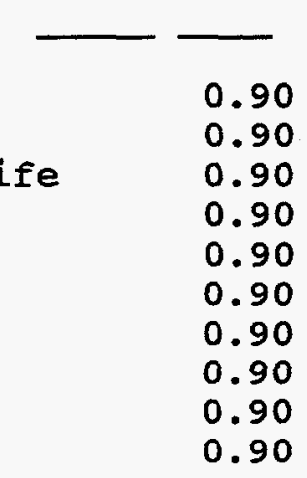



R E S O U R C E
D A T A B A S E
L I $S T I N G$

DATE: $08 / 19 / 97$

PAGE NO: 76

FERC

Number

Plant Name

stream

State

NV45518 LEHMAN PLANT

IEHMAN CREEK \& TRIBUTARIES

Name

County Name

WHITE PINE

River Basin

GREAT SALT LAKE BASIN

Class Owner Name

R BAKER RANCHES, INC.

Name Plate

Rating ( $\mathrm{KW}$ )

PESF

Annual Energy

128.00

$0.90 \quad 115.20$

487.00

NV

PESF Annual Energy Rating (MWh)

$$
438.30
$$

\begin{tabular}{|c|c|c|c|c|}
\hline $\begin{array}{l}\text { Unit } \\
\text { Type }\end{array}$ & $\begin{array}{l}\text { Plant } \\
\text { Type }\end{array}$ & $\begin{array}{l}\text { Project } \\
\text { status }\end{array}$ & $\begin{array}{l}\text { Dam } \\
\text { Status }\end{array}$ & $\begin{array}{lr}\text { Latitude } & 3901.00 \\
\text { Longitude } & 11407.00\end{array}$ \\
\hline C & DIV & $x x$ & U & \\
\hline
\end{tabular}

Factor

wild/Scenic Protection

wild/Scenic Tributary or

Upstream/Downstream

wild/Scenic Location

Cultural value

Fish Presence Value

Geologic Value

Historic Value

other Value

Recreation value

Scenic Value
Exist Prob

0.90

Wildlife Value

Threatened/Endangered Fish

Threatened/Endangered Wildlife

0.90 Federal Land Code 103

0.90 Federal Land Code 104

0.90 Federal Land Code 105

0.90 Federal Land Code 106

0.90 Federal Land Code 107

0.90 Federal Land Code 108

0.90 Federal Land Code 198
Exist Prob

0.90

0.90

0.90

0.90

0.90

0.90

0.90

0.90

0.90

0.90 
FERC

Number

NV45749 VIPONT CREEK PLANT

County Name

WHITE PINE

Plant Name

\section{Class}

$\mathbf{R}$

ROBISON, REED

Name Plate

Rating (KW)

65.00

PESF

PESF*KW

Annual Energy

Rating (MWh)

252.00

$0.90 \quad 58.50$

\begin{abstract}
Plant
Type

ROR
\end{abstract}

Type

C

Factor

Exist Prob

0.90

Wildlife Value Threatened/Endangered Fish Threatened/Endangered Wildlife

0.90 Federal Land Code 103

0.90 Federal Land Code 104

0.90 Federal Land Code 105

0.90 Federal Land Code 106

0.90 Federal Land Code 107

0.90 Federal Land Code 108

0.90 Federal Land Code 198
PESF Annual Energy Rating (MWh) 226.80
State Name

NV
wild/Scenic Protection

Upstream/Downstream Cultural Value

Fish Presence Value

Geologic Value

Historic Value

other value

Recreation value

Scenic Value
0.90 
DATE: $08 / 19 / 97$

PAGE NO: 78

FERC

Number

Plant Name

Stream

State

NV45750 CLEVELAND RANCH PLANT

CLEVE CREEK

Name

county Name

River Basin

WHITE PINE

SPRING BASIN

Class Owner Name

R RDBISON, REED B., ET AL

Name Plate

Rating ( $\mathrm{KW}$ )

PESF

Annual Energy

$\begin{array}{lll}115.00 & 0.90 \quad 103.50\end{array}$

433.00

NV

PESF Annual Energy Rating (MWh)

$\begin{array}{ccccc}\text { Unit } & \text { Plant } & \text { Project } & \text { Dam } & \text { Latitude } \\ \text { Type } & \text { Type } & \text { Status } & \text { Status } & \text { Longitude } 11430.00 \\ \text { C } & \text { DIV } & X X & U & \end{array}$

Factor

Wild/Scenic Protection

Wild/Scenic Tributary or Upstream/ Downstream wild/Scenic Location Cultural value Fish Presence Value Geologic Value Historic value Other Value Recreation Value Scenic Value
Exist Prob

0.90

Wildlife Value Threatened/Endangered Fish Threatened/Endangered Wildlife

0.90 Federal Land Code 103

0.90 Federal Land Code 104

0.90 Federal Land Code 105

0.90 Federal Land Code 106

0.90 Federal Land Code 107

0.90 Federal Land Code 108

0.90 Federal Land Code 198
Exist Prob

0.90

0.90

0.90

0.90

0.90

0.90

0.90

0.90

0.90

0.90 
R E S O U R C E

DATE: $08 / 19 / 97$
D A T A B A S E

I I S T I N G

PAGE NO: 79

FERC

Number

Plant Name

NV49555 RED POINT PLANT

county Name

ELKO

stream

state

Name

THOUSAND SPRINGS CREEK

NV

River Basin

GREAT SALT LAKE BASIN

Class Owner Name

$R$ IANDS OF SIERRA, INC.

Name Plate

Rating (KW)

PESF

Annual Energy

PESF*KW

Rating (MWh)

PESF Annual

76.00

$0.90 \quad 68.40$

277.00

249.30

Unit Plant

Type Type

Project

Dam

status

Iatitude

4128.00

C

STG

XX

U

Factor

Exist Prob

Factor

Exist Prob

wild/Scenic Protection

wild/Scenic Tributary or

0.90 Wildlife Value

Threatened/Endangered Fish

Threatened/Endangered Wilalife

Upstream/Downstream

wild/Scenic Location

Cultural Value

Fish Presence Value

$\begin{array}{ll}0.90 & \text { Federal Land Code } 103 \\ 0.90 & \text { Federal Land Code } 104\end{array}$

0.90 Federal Iand code 105

0.90 Federal Land code 106

0.90 Federal Land Code 107

0.90 Federal Land Code 108

0.90 Federal Land Code 198

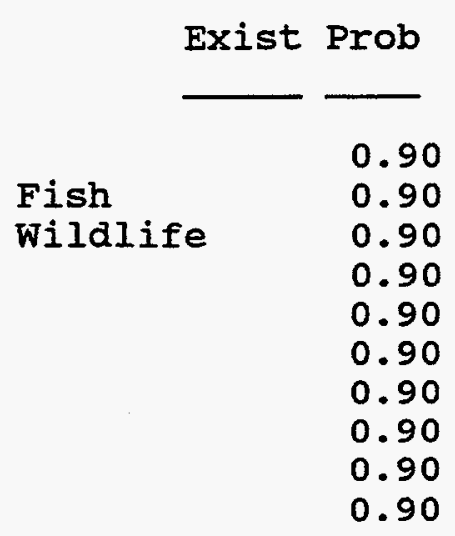

0.90

other Value
Recreation Value

Scenic Value 
DATE: $08 / 19 / 97$

PAGE NO: 80

FERC

Number

Plant Name

NV49556 ECCLES PLANT

County Name

River Basin

ELKO

GREAT SALT LAKE BASIN

Class Owner Name

$R$ LALNDS OF SIERRA, INC.

Name Plate

Rating (KW)

76.00
PESF

$0.90 \quad 68.40$

Plant

Type

C

STG
Project status

$2 x x$
Annual Energy $\mathrm{PESF} * \mathrm{KW}$ Rating (MWh)

277.00

State

Name

NV

\begin{tabular}{|c|c|c|c|c|}
\hline $\begin{array}{l}\text { Unit } \\
\text { Type }\end{array}$ & $\begin{array}{l}\text { Plant } \\
\text { Type }\end{array}$ & $\begin{array}{l}\text { Project } \\
\text { status }\end{array}$ & $\begin{array}{l}\text { Dam } \\
\text { status }\end{array}$ & $\begin{array}{lr}\text { Latitude } & 4134.00 \\
\text { Longitude } & 11425.00\end{array}$ \\
\hline C & STG & $2 x x$ & U & \\
\hline
\end{tabular}

\section{Factor}

Wild/Scenic Protection Wild/Scenic Tributary or Upstream/Downstream wild/Scenic Location Cultural value Fish Presence Value Geologic Value Historic Value other Value Recreation Value Scenic Value
Exist Prob

0.90

Wildlife Value Threatened/Endangered Fish Threatened/Endangered Wildlife

0.90 Federal Land Code 103

0.90 Federal Land Code 104

0.90 Federal Land Code 105

0.90 Federal Land Code 106

0.90 Federal Land Code 107

0.90 Federal Land Code 108

0.90 Federal Land Code 198
PESF Annual Energy Rating (MWh)

249.30 
DATE: $08 / 19 / 97$

PAGE NO: 81

FERC

Number

Plant Name

NV80000 26 FOOT DROP POWER PLANT

County Name

CHURCHILL
State

Name

$\checkmark$ CANAL

River Basin

CARSON RIVER BASIN

NV

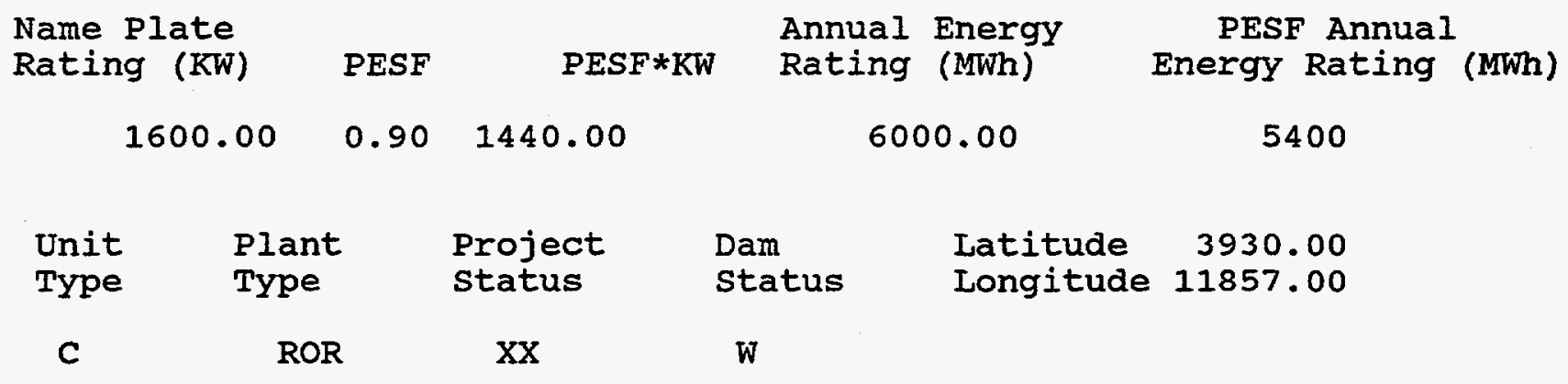

Factor

Wild/Scenic Protection

Wild/Scenic Tributary or Upstream/Downstream wild/Scenic Location

Cultural Value

Fish Presence Value

Geologic Value

Historic Value

other Value

Recreation Value

Scenic Value
Exist Prob

Factor

Exist Prob

$\begin{array}{ll}0.90 & \text { Wildife Value } \\ & \text { Threatened/Endangered Fish } \\ & \text { Threatened/Endangered Wildlife } \\ 0.90 & \text { Federal Land Code } 103 \\ 0.90 & \text { Federal Land Code } 104 \\ 0.90 & \text { Federal Land Code } 105 \\ 0.90 & \text { Federal Land Code } 106 \\ 0.90 & \text { Federal Land Code } 107 \\ 0.90 & \text { Federal Land Code } 108 \\ 0.90 & \text { Federal Land Code } 198 \\ 0.90 & \end{array}$

0.90

0.90

0.90

0.90

0.90

0.90

0.90

0.90

0.90

0.90 
R E S O U R C E D A T A B A S E I I S T I N G

DATE: $08 / 19 / 97$

PAGE NO: 82

FERC

Number

Plant Name

Stream

state

NVHOOO2 OVERTON PLANT

VIRGIN RIVER

Name

NV

county Name

CLARK
River Basin

COLORADO RIVER BASIN \begin{tabular}{cc} 
Class & \multicolumn{2}{c}{ Owner Name } \\
C & U.S.D.I. BUREAU OF RECLAMATION
\end{tabular}

Name Plate

Rating (KW)

PESF

Annual Energy

PESF*KW Rating (MWh)

PESF Annual Energy Rating (MWh)

430.00

$0.50 \quad 21.5 .00$

1537.00

768.50

$\begin{array}{cccllr}\text { Unit } & \text { Plant } & \text { Project } & \text { Dam } & \text { Latitude } & 3638.00 \\ \text { Type } & \text { Type } & \text { Status } & \text { Status } & \text { Longitude } 11419.00 \\ \text { C } & \text { ROR } & \text { XX } & \text { U } & \end{array}$

\author{
Factor \\ Wild/Scenic Protection \\ wild/Scenic Tributary or \\ Upstream/Downstream \\ wild/Scenic Iocation \\ cultural value \\ Fish Presence Value \\ Geologic Value \\ Historic Value \\ other Value \\ Recreation Value \\ scenic Value
}

Factor

Exist: Prob

0.90

Wildlife Value Threatened/Endangered Fish Threatened/Endangered Wildlife

0.90 Federal Land code 103

0.90 Federal Land code 104

0.90 Federal Land code 105

0.90 Federal Land Code 106

0.90 Federal Iand code 107

$Y \quad 0.50$

0.90

0.90

Federal Land Code 198
Exist Prob

0.90

0.90

0.90

0.90

0.90

0.90

0.90

0.90

0.90

0.90 

$\begin{array}{llllllll}R & E & S & O & U & R & C & E\end{array}$
D A $T$ A B A S E
L I S T I G

DATE : $08 / 19 / 97$

PAGE NO: 83

FERC

Number

Plant Name

stream

State

Name

NVH0004 CLEAR CREEK

CLEAR CREEK

NV

County Name

River Basin

DOUGLAS

CARSON RIVER BASIN

Class Owner Name

R UNKNOWN

Name Plate

Annual Energy

Rating (MWh)

PESF Annual

Rating (KW)

PESF

PESF*KW

Energy Rating (MWh)

$$
198.00
$$

$0.25 \quad 49.50$

Plant

Project

Type

Type

status

C

ROR

$\mathrm{XX}$

Factor

Exist Prob

wild/Scenic Tributary or Upstream/Downstream wild/Scenic Location

Cultural value

Fish Presence Value

Geologic Value

Historic Value

other value

Recreation Value

Scenic Value

\subsection{0}

Exist Prob

0.90 Federal Land Code 103

0.90 Federal Land Code 104

0.90 Federal Land Code 105

0.90 Federal Land Code 106

0.90 Federal Land Code 107

0.90 Federal Land Code 108

$\mathrm{Y} \quad 0.25$

0.90
0.90

0.90

0.90

0.90

0.90

0.90

0.90

0.90

0.90

0.90
748.00

Dam

Status

Latitude $\quad 3907.00$

U

Wildlife Value

Threatened/Endangered Fish

Threatened/Endangered Wildlife

187

Federal Land Code 198 
DATE: $08 / 19 / 97$

FERC

Number

Plant Name

NVHO006 BRYANT CREEK

County Name

DOUGLAS

Class

F

U.S.D.I.

Owner Name

Name Plate

Rating (KW)

PESF

PESF*KW

Annual Energy

Rating (MWh)

PAGE NO: 84

BRYANT CREEK

River Basin

CARSON RIVER BASIN state

Name

NV

$$
104.00 \quad 0.50 \quad 52.00
$$

388.00

PESF Annual Energy Rating (MWh)

194

$\begin{array}{ccccc}\text { Unit } & \text { Plant } & \text { Project } & \text { Dam } & \text { Latitude } \\ \text { Type } & \text { Type } & \text { Status } & \text { Status } & \text { Longitude } 11942.00 \\ \text { C } & \text { ROR } & X X & \text { U } & \end{array}$

Factor

Exist Prob

Factor

Exist Prob

Wild/Scenic Protection

wild/Scenic Tributary or

0.90 Wildlife value

0.90

Upstream/ Downstream

Threatened/Endangered Fish

0.90

wild/Scenic Location Threatened/Endangered Wildlife

0.90

0.90 Federal Land code 103

0.90

0.90 Federal Land Code 104

0.90

0.90 Federal Land Code 105

0.90

Fish Presence Value

0.90 Federal Land Code 106

0.90

Geologic Value

0.90 Federal Land Code 107

$\mathbf{Y}$

0.50

other Value

0.90 Federal Land Code 108

0.90

Recreation Value

0.90 Federal Land Code 198

0.90

0.90 
R E S O U R E

DATE : $08 / 19 / 97$
D A T A B A S E

FERC

Number

Plant Name

NVHO008 SMITH VALLEY

County Name

DOUGLAS COUNTY

class Owner Name

F U.S. FOREST SERVICE

Name Plate

Rating ( $K W)$

PESF

PESF*KW

Annual Energy

Rating (MWh)

1625.00

PAGE NO: 85

$$
427.00
$$

0.50

213.50

Plant

Project

status

Dam
status

Latitude

Longitude 11920.

Type

ROR

$\mathrm{XX}$

$\mathrm{U}$

Factor

Wild/Scenic Protection

Wild/Scenic Tributary or

Upstream/Downstream

wild/Scenic Location

Cultural Value

Fish Presence Value

Geologic Value

Historic Value

other value

Recreation Value

Scenic Value
Exist Prob

0.90

Wildife Value

Threatened/Endangered Fish

Threatened/Endangered Wildlife

0.90 Federal Land Code 103

0.90 Federal Land Code 104

0.90 Federal Land code 105

0.90 Federal Land Code 106

0.90 Federal Land Code 107

0.90 Federal Land Code 108

0.90 Federal Land Code 198
PESF Annual Energy Rating (MWh)

812.50
State

Name

NV 
RESOURCE D A T A B A S E I I S T I N G

DATE: $08 / 19 / 97$

PAGE NO: 86

FERC

Number

Plant Name

stream

State

Name

NVHOOO9 CHICKEN CREEK SUMMIT

JACK CREEK

NV

County Name

River Basin

ELKO

SNAKE RIVER BASIN

Class Owner Name

R UNKNOWN

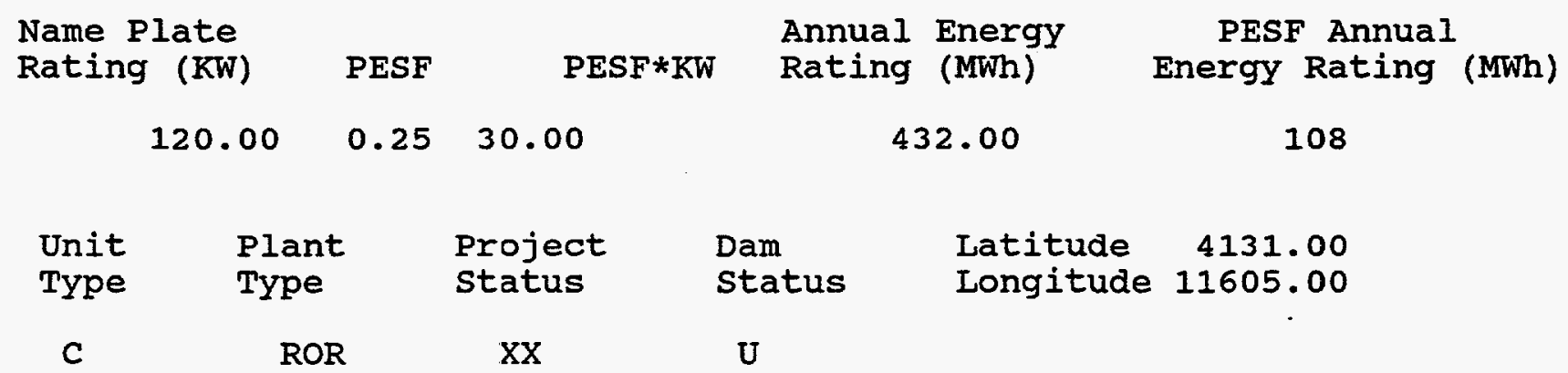

Factor

Exist Prob

Factor

Exist Prob

Wild/scenic Protection

Wild/Scenic Tributary or

0.90 Wildlife Value

0.90

Threatened/Endangered Fish

Upstream/Downstream

wild/Scenic Location

Threatened/Endangered Wildlife

0.90

0.90

0.90 Federal Land Code 103

0.90

0.90 Federal Land Code 104

0.90

0.90 Federal Land Code 105

0.90

0.90 Federal Land Code 106

0.90

0.90 Federal Land Code 107

0.90

Historic Value

0.90 Federal Land Code 108

0.90

Recreation value

0.25 Federal Land Code 198

0.90

Scenic Value

0.90 
DATE: $08 / 19 / 97$

FERC

Number

Plant Name

NVHO011 ROWLAND PLANT

county Name

ELRO
PAGE NO: 87

State

Name

NV
BRUNEAU RIVER

River Basin

SNAKE RIVER BASIN

\section{Class Owner Name \\ F U.S. FOREST SERVICE}

\begin{abstract}
Name Plate Rating (KW)

PESF

Annual Energy

$P E S F * K W$

Rating (MWh)
\end{abstract}

$0.10 \quad 61.70$

617.00

PESF Annual

Energy Rating (MWh)

$$
2211.00
$$

221.10

Factor

Wild/Scenic Protection

Wild/scenic Tributary or

Upstream/Downstream

wild/Scenic Location

Cultural value

Fish Presence Value

Geologic Value

Historic Value

other Value

Recreation Value

Scenic Value

\begin{abstract}
Project Status
\end{abstract}

$\mathrm{XX}$

\section{Dam \\ Status}

$\mathrm{U}$
Exist Prob

Factor

Latitude $\quad 4154.00$

Longitude 11530.00

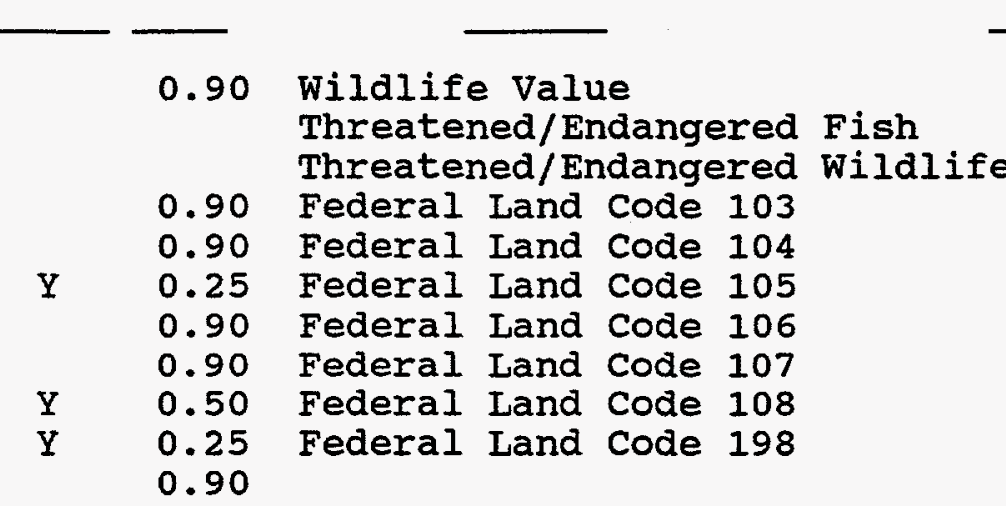

Exist Prob

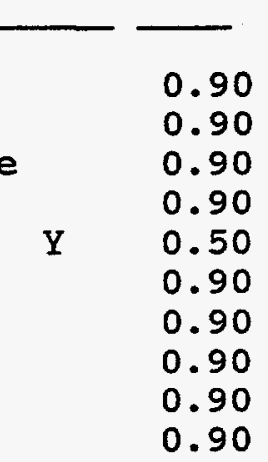


DATE: $08 / 19 / 97$

PAGE NO: 88

FERC

Number

Plant Name

NVHOO14 HOT SPRING CREEK PLANT

county Name

ELKO

Class

C UNKNOWN

Name Plate

Rating ( $\mathrm{KW})$

PESF

PESF*KW

Annual Energy

Rating (MWh)

$$
\begin{array}{lll}
56.00 & 0.50 \quad 28.00
\end{array}
$$

201.00

MARY'S RIVER

stream

State

Name

NV
River Basin

HUMBOLDT RIVER BASIN

\begin{tabular}{|c|c|c|c|c|}
\hline $\begin{array}{l}\text { Unit } \\
\text { Type }\end{array}$ & $\begin{array}{l}\text { Plant } \\
\text { Type }\end{array}$ & $\begin{array}{l}\text { Project } \\
\text { status }\end{array}$ & $\begin{array}{l}\text { Dam } \\
\text { status }\end{array}$ & $\begin{array}{lr}\text { Latitude } & 4115.00 \\
\text { Longitude } & 11515.00\end{array}$ \\
\hline C & ROR & $x x$ & $\mathrm{U}$ & \\
\hline
\end{tabular}

Factor

Wild/Scenic Protection

wild/Scenic Tributary or Upstream/Downstream wild/Scenic Location Cultural value Fish Presence Value Geologic Value Historic Value other Value Recreation Value Scenic Value
Exist Prob
PESF Annual Energy Rating (MWh) 100.50 

$\begin{array}{llllllll}\text { R } & \text { E } & S & \text { O } & \text { U } & R & \text { C } & \text { E }\end{array}$
D A T A B A S E
L I S T I N G

DATE : $08 / 19 / 97$

PAGE NO: 89

FERC

Number

Plant Name

stream

State

Name

NVH0015 HERDER PILANT

HERDER CREEK

NV

County Name

River Basin

EIKO

HUMBOIDT RIVER BASIN

class Owner Name

R UNKNOWN

Name Plate

Rating ( $\mathrm{KW})$

PESF

Annual Energy

PESF Annual

126.00

$0.90 \quad 113.40$

470.00

423

\begin{tabular}{|c|c|c|c|c|c|}
\hline $\begin{array}{l}\text { Unit } \\
\text { Type }\end{array}$ & $\begin{array}{l}\text { Plant } \\
\text { Type }\end{array}$ & $\begin{array}{l}\text { Project } \\
\text { status }\end{array}$ & $\begin{array}{l}\text { Dam } \\
\text { status }\end{array}$ & $\begin{array}{l}\text { Latitude } \\
\text { Longitude }\end{array}$ & $\begin{array}{r}4102.00 \\
11511.00\end{array}$ \\
\hline$c$ & ROR & $\mathrm{XX}$ & $\mathrm{U}$ & & \\
\hline
\end{tabular}

Factor

Exist Prob

Factor

Exist Prob

Wild/Scenic Protection

wild/Scenic Tributary or

0.90 Wildlife Value
Threatened/Endangered Fish Threatened/Endangered Wildlife

0.90

Upstream/Downstream wild/Scenic Location

0.90 Federal Land code 103

0.90

0.90 Federal Land code 104

0.90

Cultural Value

0.90 Federal Land code 105

0.90

Federal Land code 106

0.90

Fish Presence Value

0.90

Federal Land Code 107

0.90

0.90

0.90

0.90

Federal Land code 108

0.90

other Value

$\begin{array}{ll}\text { Recreation Value } & 0.90 \\ \text { Scenic Value } & 0.90\end{array}$

Federal Land Code 198

0.90

$\begin{array}{ll}\text { Recreation Value } & 0.90 \\ \text { Scenic Value } & 0.90\end{array}$ 
RESOURC $\mathrm{E}$ D D T A B A S E I I S T I N G

DATE: $08 / 19 / 97$

PAGE NO: 90

FERC

Number

Plant Name

NVHO016 TENT MOUNTAIN \#1

County Name

River Basin

ELKO

HUMBOLDT RIVER BASIN

\section{class Owner Name}

R UNKNOWN

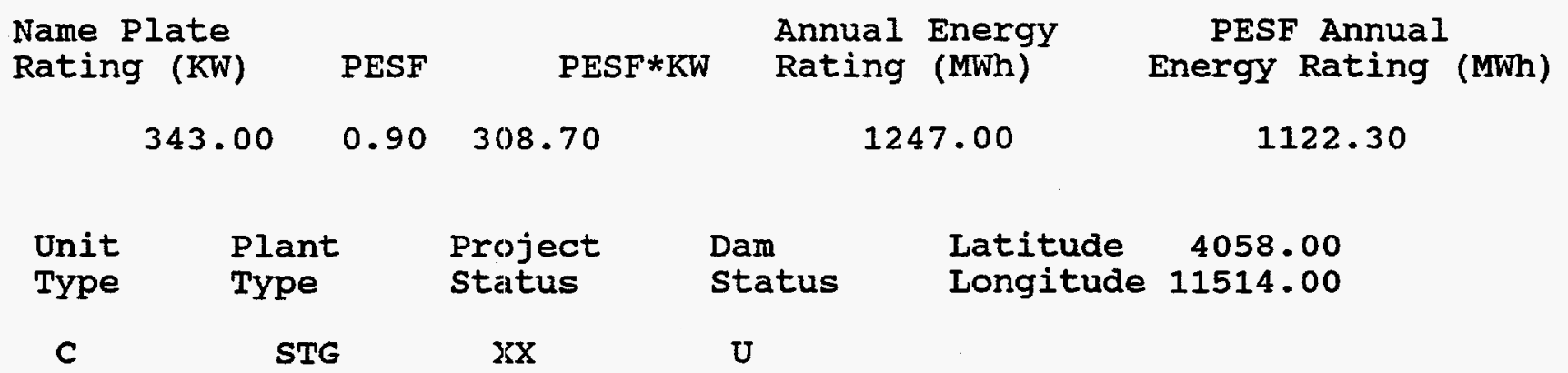

Factor

Wild/Scenic Protection

Wild/Scenic Tributary or Upstream/Downstream wild/Scenic Location Cultural value

Fish Presence Value

Geologic Value

Historic Value

other Value

Recreation value

Scenic Value
Exist: Prob

0.90

Wildife Value

Threatened/Endangered Fish

Threatened/Endangered Wildlife

0.90 Federal Land Code 103

0.90 Federal Land Code 104

0.90 Federal Land Code 105

0.90 Federal Land Code 106

0.90 Federal Land Code 107

0.90 Federal Land Code 108

0.90 Federal Land Code 198

0.90
State

Name

NV 

RESOU R C E
D A T A B A S E
L I S T I N G

DATE: $08 / 19 / 97$

PAGE NO: 91

FERC

Number

Plant Name

Stream

State

NVHO017 TENT MOUNTAIN \#2

WEST FORK BOULDER CREEK

Name

NVHOO17 TENT MOUNTAIN \#2

county Name

River Basin

ELKO

HUMBOLDT RIVER BASIN

Class Owner Name

R UNKNOWN

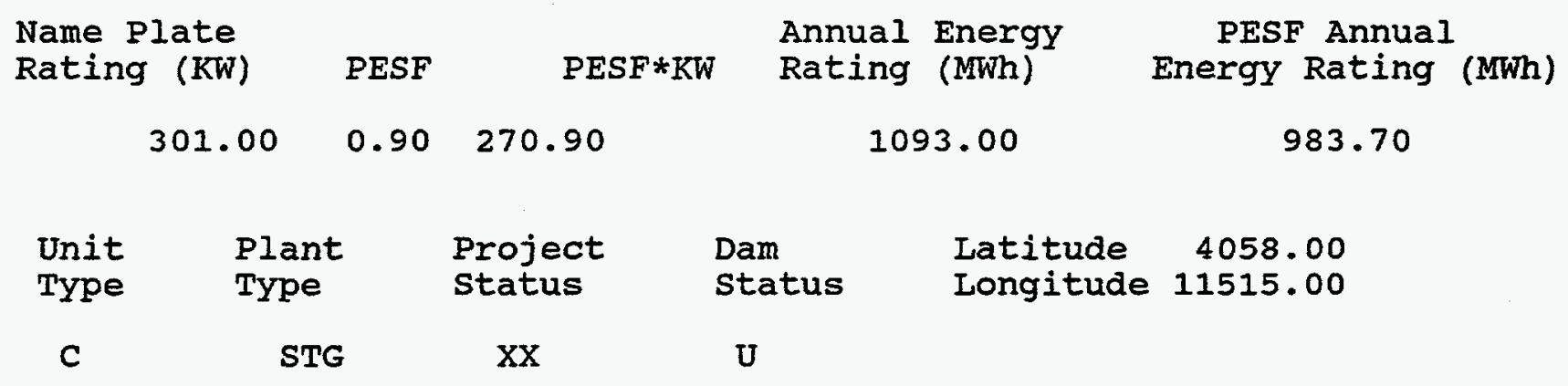

Factor

Exist Prob

Factor

Exist Prob

wild/scenic Protection

wild/Scenic Tributary or

Upstream/ Downstream

wild/Scenic Location

Cultural value

Fish Presence Value

Geologic Value

Historic Value

other Value

Recreation value

0.90

wildlife value

Threatened/Endangered Fish

Threatened/Endangered Wildlife

NV

NV

0.90 Federal Land code 103

0.90 Federal Land Code 104

0.90 Federal Land code 105

0.90 Federal Land Code 106

0.90 Federal Land Code 107

0.90 Federal Land Code 108

0.90 Federal Land Code 198

0.90

0.90

0.90

0.90

0.90

0.90

0.90

0.90

0.90

0.90

0.90 
RESOURCE DATABASE L I S T I NG

DATE : $08 / 19 / 97$

PAGE NO: 92

FERC

Number

Plant Name

NVHO018 SECRET PLANT

County Name

River Basin

ELKO

HUMBOLDT RIVER BASIN

Class Owner Name

R UNKNOWN

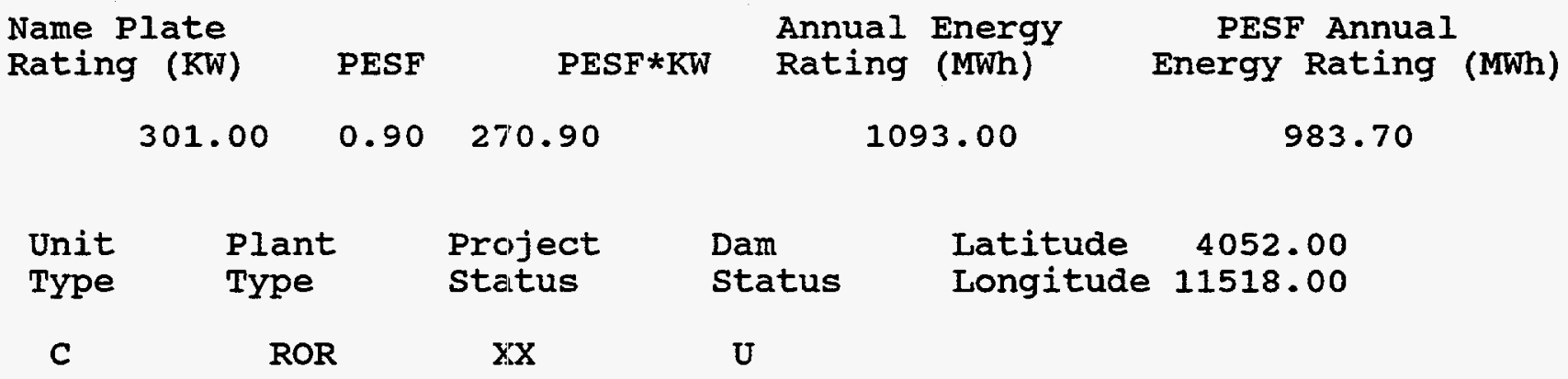

Factor

Exist. Prob

Factor

Exist Prob
Wild/Scenic Protection

wild/Scenic Tributary or Upstream/Downstream wild/Scenic Location Cultural value Fish Presence Value Geologic Value Historic Value other Value Recreation value Scenic Value
0.90 Wildlife Value Threatened/Endangered Fish Threatened/Endangered Wildlife

0.90 Federal Land Code 103

0.90 Federal Land Code 104

0.90 Federal Land Code 105

0.90 Federal Land Code 106

0.90 Federal Land code 107

0.90 Federal Land Code 108

0.90 Federal Land Code 198 state Name

NV

0.90

0.90

0.90

0.90

0.90

0.90

0.90

0.90

0.90

0.90

0.90 
DATE: $08 / 19 / 97$

FERC

Number

Plant Name

NVH0019 TENT MOUNTAIN \#3

County Name

ELKO

Class

Owner Name

R UNKNOWN

Name Plate

Rating ( $\mathrm{KW})$

PESF

Annual Energy Rating (MWh)

743.00

Dam

Unit

Plant

Type

Type

C

ROR

Factor

Exist Prob

Factor

Latitude $\quad 4101.00$

status

$\mathrm{XX}$

status

Longitude 11512.00
State

Name

NV
PESF Annual Energy Rating (MWh)

668.70
Wild/Scenic Protection

wild/Scenic Tributary or Upstream/Downstream wild/Scenic Location Cultural value

Fish Presence Value

Geologic Value

Historic Value

other Value

Recreation Value

Scenic Value
0.90 Wildlife Value

Threatened/Endangered Fish Threatened/Endangered Wildlife

0.90 Federal Land Code 103

0.90 Federal Land Code 104

0.90 Federal Land Code 105

0.90 Federal Land Code 106

0.90 Federal Land Code 107

0.90 Federal Land Code 108

0.90 Federal Land Code 198

0.90

Exist Prob

0.90

0.90

0.90

0.90

0.90

0.90

0.90

0.90

0.90

0.90 
DATE: $08 / 19 / 97$

PAGE NO: 94

FERC

Number

Plant Name

Stream

State

NVHOO21 TALBOT PLANT

TALBOT CREER

Name

NV

County Name

ELKO

Class

C UNNKNOWN

Name Plate

Rating (KW)

PESF

$\mathrm{PESF} * \mathrm{KW}$

Annual Energy

Rating (MWh)

1402.00

River Basin

HUMBOLDT RIVER BASIN
$382.00 \quad 0.25 \quad 95.50$

Unit

Type

C

$$
\begin{aligned}
& \text { Plant } \\
& \text { Type } \\
& \text { ROR }
\end{aligned}
$$

Project status

$\mathrm{XX}$
Exist Prob
Dam
Status

U
PESF Annual Energy Rating (MWh)

\footnotetext{
Factor

Wild/Scenic Protection

wild/Scenic Tributary or

Upstream/Downstream

wild/Scenic Location

Cultural value

Fish Presence Value

Geologic Value

Historic Value

other value

Recreation value

Scenic Value
}

Factor

Exist Prob

350.50 
DATE: $08 / 19 / 97$

FERC

Number

Plant Name

NVHO022 RYNDON PLANT

County Name

ELKO

Class Owner Name

C UNKNOWN

PAGE NO: 95

State

Name

HUMBOLDT RIVER

NV

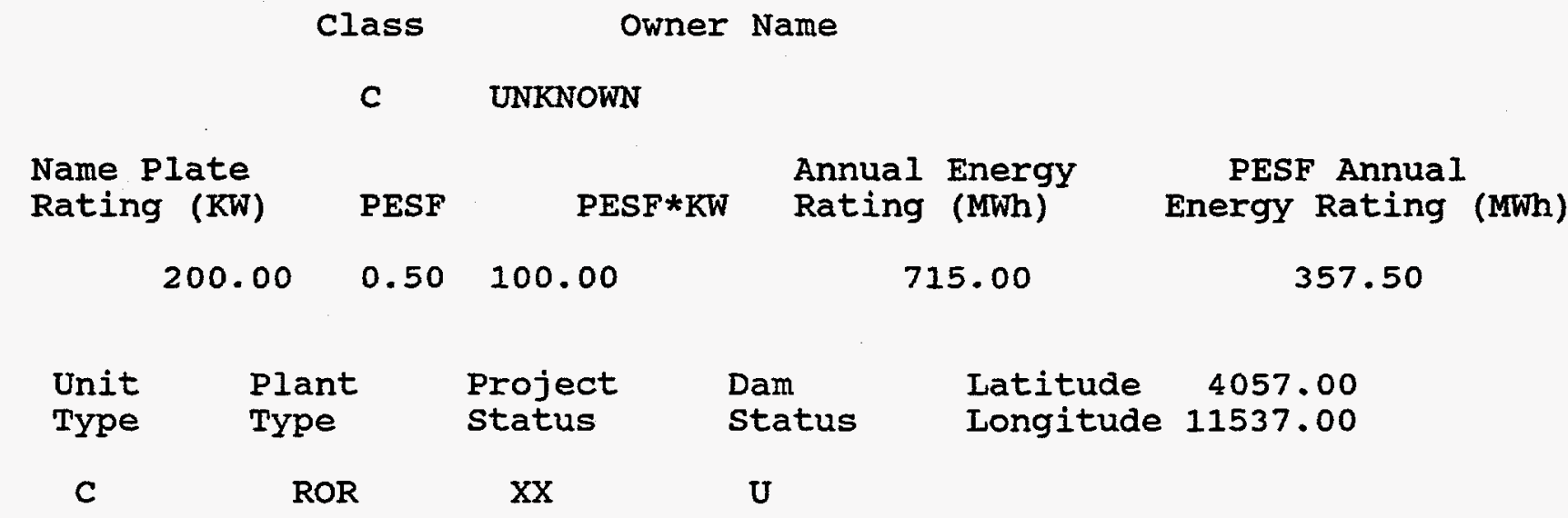

Factor

Exist Prob

Factor

Exist Prob

Wild/Scenic Protection

Wild/Scenic Tributary or

Upstream/Downstream

wild/Scenic Location

HUMBOLDT RIVER BASIN

River Basin

Cultural value

Fish Presence Value

Geologic Value

Historic Value

other Value

Recreation Value

Scenic Value

$\begin{array}{ll}0.90 & \begin{array}{l}\text { Wildife Value } \\ \text { Threatened/Endangered Fish } \\ \text { Threatened/Endangered Wildlife }\end{array} \\ 0.90 & \text { Federal Land Code } 103 \\ 0.90 & \text { Federal Land Code } 104 \\ 0.90 & \text { Federal Land Code } 105 \\ 0.90 & \text { Federal Land Code } 106 \\ 0.90 & \text { Federal Land Code } 107 \\ 0.50 & \text { Federal Land Code } 108 \\ 0.90 & \text { Federal Land Code } 198 \\ 0.90 & \end{array}$

0.90

0.90

0.90

0.90

0.90

0.90

0.90

0.90

0.90

0.90 
RE SOURCE DA T A B A S E I I S T I N G

DATE: $08 / 19 / 97$

PAGE NO: 96

FERC

Number

Plant Name

Stream

state

NVHO023 THORPE PLANT

THORPE CREEK

Name

NV

county Name

River Basin

ELKO

HUMBOLDT RIVER BASIN

Class Owner Name

R UNKNOWN

Name Plate

PESF $\quad$ PESF*KW $\begin{aligned} & \text { Annual Energy } \\ & \text { Rating (MWh) }\end{aligned}$

PESF Annual

Rating ( $\mathrm{KW})$

$0.90 \quad 219.60$

Energy Rating (MWh)

244.00

Unit Plant

Type Type

C

ROR

Factor

Exist Prob

Wild/Scenic Protection

wild/Scenic Tributary or Upstream/Downstream wild/Scenic Location Cultural Value

Fish Presence Value

Geologic Value

Historic Value

other Value

Recreation Value

Scenic value
890.00

Project st:atus

$\mathrm{xx}$

Dam

Status

Latitude

Langitude 11526.00

801

$\mathrm{U}$
0.90

Factor

Exist Prob

wildlife Value

Threatened/Endangered $\mathrm{F}$ ish

Threatened/Endangered Wildife

0.90 Federal Land Code 103

0.90 Federal Land Code 104

0.90 Federal Land code 105

0.90 Federal Land code 106

0.90 Federal Land Code 107

0.90 Federal Land code 108

0.90 Federal Land Code 198
0.90

0.90

0.90

0.90

0.90

0.90

0.90

0.90

0.90

0.90 
DATE: $08 / 19 / 97$

FERC

Number

Plant Name

NVH0026 ADOBIE PLANT

County Name

ELKO
PAGE NO: 97

State

Name

SUSIE CREEK

NV

River Basin

HUMBOLDT RIVER BASIN

\section{Class Owner Name}

R UNKNOWN

\begin{tabular}{|c|c|c|c|c|c|c|}
\hline $\begin{array}{l}\text { Name } P \\
\text { Rating }\end{array}$ & $\begin{array}{l}\text { Late } \\
(K W)\end{array}$ & PESF & $\mathrm{PESF} * \mathrm{KW}$ & $\begin{array}{l}\text { Anny } \\
\text { Rati }\end{array}$ & $\begin{array}{l}\text { Energy } \\
\text { (MWh) }\end{array}$ & $\begin{array}{l}\text { PESF Annual } \\
\text { Energy Rating (MWh) }\end{array}$ \\
\hline & 49.00 & 0.90 & 44.10 & & 96.00 & 176.40 \\
\hline $\begin{array}{l}\text { Unit } \\
\text { Type }\end{array}$ & $\begin{array}{l}\text { Pla } \\
\text { TYl }\end{array}$ & & $\begin{array}{l}\text { Project } \\
\text { Status }\end{array}$ & $\begin{array}{l}\text { Dam } \\
\text { status }\end{array}$ & $\begin{array}{l}\text { Latitude } \\
\text { Longitude }\end{array}$ & $\begin{array}{r}4050.00 \\
11602.00\end{array}$ \\
\hline C & & & $\mathrm{xx}$ & $\mathbf{U}$ & & \\
\hline
\end{tabular}

Factor

Wild/Scenic Protection

wild/Scenic Tributary or

Upstream/Downstream

wild/Scenic Location

Cultural value

Fish Presence Value

Geologic Value

Historic Value

other Value

Recreation value

Scenic Value
Exist Prob

Factor

Exist Prob

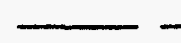

0.90

Wildife Value Threatened/Endangered Fish Threatened/Endangered Wildlife

0.90 Federal Land Code 103

0.90 Federal Land Code 104

0.90 Federal Land Code 105

0.90 Federal Land Code 106

0.90 Federal Land Code 107

0.90 Federal Land Code 108

0.90 Federal Land Code 198

0.90
0.90
0.90
0.90
0.90
0.90
0.90
0.90
0.90
0.90


R E S O U R C E D A T A B A S E I I S T I N G

DATE: $08 / 19 / 97$

PAGE NO: 98

FERC

Number

Plant Name

Stream

State

NVHO027 MAGGIE NARROWS

MAGGIE CREEK

Name

NV

County Name

River Basin

EUREKA

HUMBOLDT RIVER BASIN

Class Owner Name

R UNKNOWN

Name Plate

$\begin{array}{ll}\text { PESF } & \text { PESF*KW } \\ \text { Rating (MWh) }\end{array}$

PESF Annual

Rating (KW)

$0.25 \quad 277.50$

2538.00

Energy Rating

(MWh)

710.00

634.50

$\begin{array}{llllr}\text { Unit } & \text { Plant } & \text { Project } & \text { Dam } & \text { Latitude } \\ \text { Type } & \text { Type } & \text { Status } & \text { Status } & \text { Longitude } 11612.00\end{array}$

C

ROR

$\mathrm{XX}$

$\mathrm{U}$

Factor

Wild/Scenic Protection

Wild/Scenic Tributary or

Upstream/Downstream

wild/Scenic Location

Cultural value

Fish Presence Value

Geologic Value

Historic Value

other Value

Recreation Value

Scenic value
Exist Prob

0.90

Wildlife Value Threatened/Endangered Fish Threatened/Endangered Wildlife

0.90 Federal Land code 103

0.90 Federal Land Code 104

0.90 Federal Land Code 105

$Y \quad 0.50$ Federal Land code 106

0.90 Federal Land Code 107

0.90 Federal Land Code 108

0.90 Federal Land code 198
Exist Prob

0.90

0.90

0.90

0.90

0.90

0.90

0.90

0.90

0.90

0.90 
DATE: $08 / 19 / 97$

FERC

Number

Plant Name

NVHO028 OVERLAND PLANT

County Name

ELKO

Class

R

UNKNOWN
PAGE NO: 99

State

Name

OVERLAND CREEK

NV

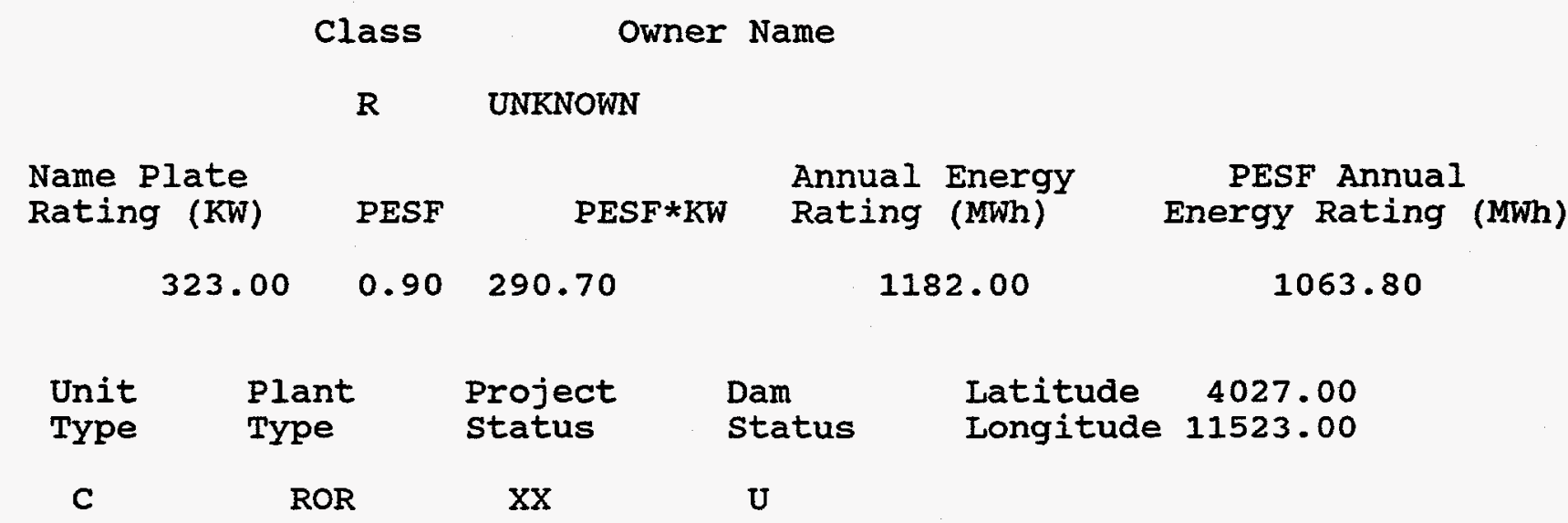

Factor

Wild/Scenic Protection

Wild/Scenic Tributary or

Upstream/Downstream

wild/Scenic Location

Cultural Value

Fish Presence Value

Geologic Value

Historic Value

other Value

Recreation Value

Scenic Value
Exist Prob

Factor

Exist Prob

0.90

wildlife Value

Threatened/Endangered Fish

Threatened/Endangered Wildlife

0.90 Federal Land Code 103

0.90 Federal Land Code 104

0.90 Federal Land Code 105

0.90 Federal Land Code 106

0.90 Federal Land Code 107

0.90 Federal Land Code 108

0.90 Federal Land Code 198

0.90
0.90

0.90

0.90

0.90

0.90

0.90

0.90

0.90

0.90

0.90 

R E S O U R C E
D A T A B A S E
L I S T I N G

DATE: $08 / 19 / 97$

PAGE NO: 100

FERC

Number

Plant Name

Stream

state

Name

NVHO029 FRANKLIN LAKE PLANT

FRANKLIN RIVER

NV

county Name

ELKO

Class Owner Name

C UNKNOWN
River Basin

FRANKLIN LAKE BASIN

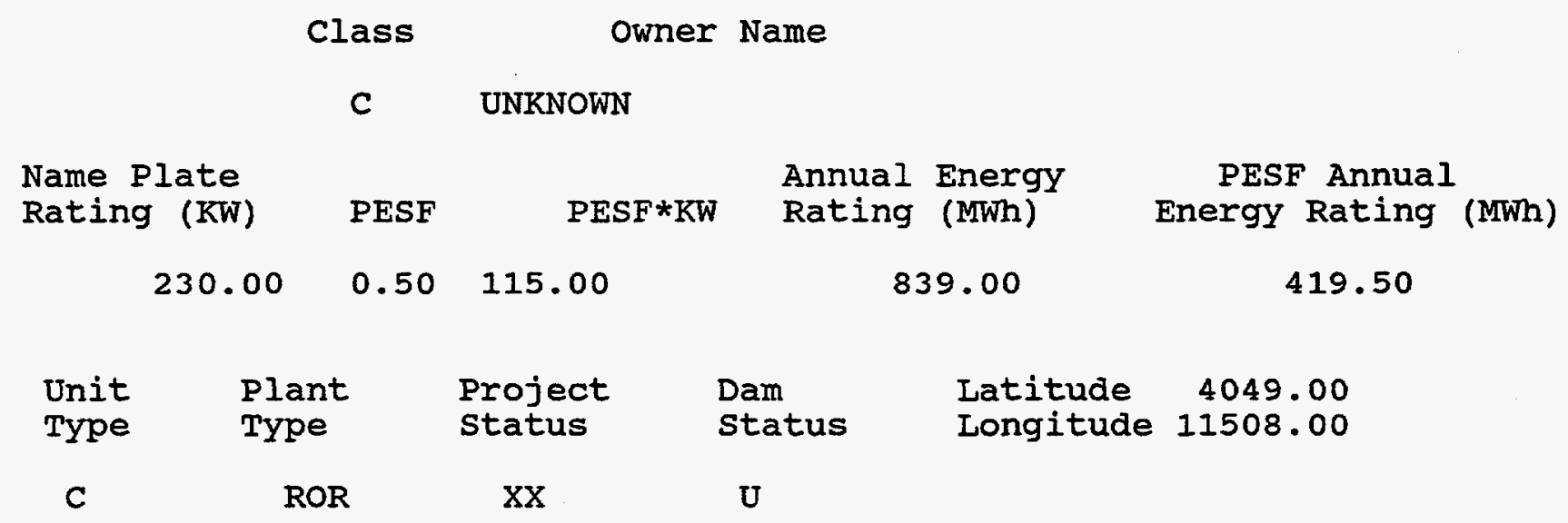

Factor

Wild/Scenic Protection Wild/Scenic Tributary or Upstream/Downstream wild/Scenic Location Cultural value Fish Presence Value Geologic Value Historic Value other Value Recreation value Scenic Value
Exist Prob

0.90

Wildlife Value Threatened/Endangered Fish Threatened/Endangered Wildilfe

0.90 Federal Land code 103

0.90 Federal Land Code 104

0.90 Federal Land Code 105

0.90 Federal Land Code 106

0.90 Federal Land Code 107

0.90 Federal Land Code 108

0.90 Federal Land code 198
Exist Prob

0.90

0.90

0.90

0.90

Y

0.50

0.90

0.90

0.90

0.90

0.90 
DATE: $08 / 19 / 97$

FERC

Number

Plant Name

NVHO031 MOUNT BARCROFT \#1

county Name

ESMERALDA

class

$\mathbf{R}$

UNKNOWN

Name Plate

Rating (KW)

$$
313.00
$$

PESF
Annual Energy

PESF*KW

Rating (MWh)

1168.00
PAGE NO: 101

State

Name

NV
River Basin

FISH LAKE BASIN

\begin{tabular}{|c|c|c|c|c|}
\hline $\begin{array}{l}\text { Unit } \\
\text { Type }\end{array}$ & $\begin{array}{l}\text { Plant } \\
\text { Type }\end{array}$ & $\begin{array}{l}\text { Project } \\
\text { Status }\end{array}$ & $\begin{array}{l}\text { Dam } \\
\text { Status }\end{array}$ & $\begin{array}{lr}\text { Latitude } & 3739.00 \\
\text { Longitude } & 11805.00\end{array}$ \\
\hline C & ROR & $X X$ & $\mathrm{U}$ & \\
\hline
\end{tabular}

Factor

Wild/Scenic Protection

Wild/Scenic Tributary or

Upstream/ Downstream

wild/Scenic Location

Cultural Value

Fish Presence Value

Geologic Value

Historic Value

other Value

Recreation value

Scenic Value
Exist Prob

0.90

Wildlife Value Threatened/Endangered Fish Threatened/Endangered Wildlife

0.90 Federal Land Code 103

0.90 Federal Land Code 104

0.90 Federal Land Code 105

0.90 Federal Land Code 106

0.90 Federal Land Code 107

0.90 Federal Land Code 108

0.90 Federal Land Code 198
PESF Annual Energy Rating (MWh)

1051.20

0.90

Exist Prob

0.90

0.90

0.90

0.90

0.90

0.90

0.90

0.90

0.90

0.90 
R E S O U R C E

DATE: $08 / 19 / 97$
D A T A B A S E

L I S T I G

PAGE NO: 102

FERC

Number

Plant Name

NVH0033 INDIAN CREEK PLANT

County Name

ESMERALDA

Class

Owner Name

C UNKNOWN

\author{
Name Plate \\ Rating (KW) \\ 167.00 \\ Factor
}

PESF

$\mathrm{PESF} * \mathrm{KW}$

Annual Energy Rating (MWh)

602.00

Latitude $\quad 3747.00$

Dam

status

Longitude 11812.00

$\begin{array}{cccll}\text { Unit } & \text { Plant } & \text { Project } & \text { Dam } & \text { Latitude } \\ \text { Type } & \text { Type } & \text { Status } & \text { Status } & \text { Longitude } 11812.00 \\ \text { C } & \text { ROR } & \mathrm{XX} & U & \end{array}$

Exist Prob

0.90

Wildife value

Threatened/Endangered Fish

Threatened/Endangered Wildlife

0.90 Federal Land Code 103

0.90 Federal Land Code 104

0.90 Federal Land Code 105

0.90 Federal Land Code 106

0.90 Federal Land Code 107

0.90 Federal Land Code 108

0.90 Federal Land Code 198
0.90
State

Name

NV
Wild/Scenic Protection

wild/scenic Tributary or Upstream/Downstream wild/Scenic Location Cultural value Fish Presence Value

Geologic Value Historic Value other Value

Recreation Value

Scenic Value
PESF Annual Energy Rating (MWh)

541.80 
FERC

Number

NVH0035 LEONARD RANCH PLANT

County Name

HUMBOLDT

Plant Name
$\mathbf{R}$

Name Plate

Rating (KW)

96.00

PESF

Annual Energy Rating (MWh)

356.00

Unit

Plant

Type

Type

C

ROR

Factor

Exist Prob

0.90

Wildlife Value

Threatened/Endangered Fish

wild/Scenic Tributary or

Upstream/Downstream

wild/Scenic Location

Cultural Value

Fish Presence Value

Geologic Value

Historic Value

other value

Recreation Value

Scenic Value
Latitude

4130.00

$\begin{array}{llr}\text { Dam } & \text { Latitude } & 4130.00 \\ \text { Status } & \text { Longitude } & 11843.00\end{array}$

U
0.90 Federal Land Code 103

0.90 Federal Land Code 104

0.90

Federal Land Code 105

0.90 Federal Land Code 106

0.90 Federal Land Code 107

0.90 Federal Land Code 108

0.90 Federal Land Code 198

\section{Wilalife}

0.90
State

Name

NV
PESF Annual Energy Rating (MWh)

320.40

Exist Prob

0.90

0.90

0.90

0.90

0.90

0.90

0.90

0.90

0.90

0.90 

R E S O U R C E
D A T A B A S E
I I S T I N G

DATE: $08 / 19 / 97$

PAGE NO: 104

FERC

Number

Plant Name

Stream

State

Name

NVH0036 TRIDENT PLANT

KING'S RIVER

NV

county Name

HUMBOLDT
River Basin

BLACK ROCK DESERT BASIN
Class Owner Name

C IJNKNOWN

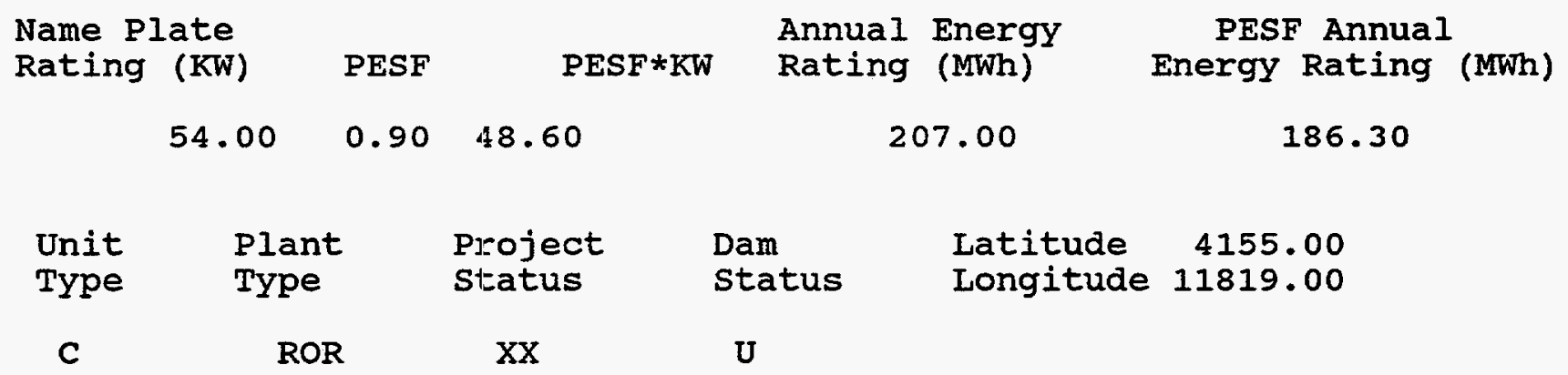

Factor

Exist Prob

Factor

Exist Prob

0.90 Wildlife Value Threatened/Endangered Fish

0.90

Wild/Scenic Protection

Wild/Scenic Tributary or Threatened/Endangered Wildlife

0.90

0.90

0.90 Federal Land code 103

0.90 Federal Land Code 104

0.90 Federal Land Code 105

0.90

0.90

0.90

0.90 Federal Land Code 106

0.90

0.90 Federal Land Code 107

0.90

0.90 Federal Land Code 108

0.90

0.90 Federal Land Code 198

0.90

0.90

Recreation Value

Scenic Value 
DATE: $08 / 19 / 97$

FERC

Number

Plant Name

NVH0037 LOG CABIN PLANT

County Name

HUMBOLDT

Class

C

UNKNOWN

Name Plate

Rating ( $\mathrm{KW}$ )

PESF

Annual Energy

43.00

$0.90 \quad 38.70$

$\mathrm{PESF} * \mathrm{KW}$

Rating (MWh)

181.00

Dam Latitude $\quad 4155.00$

Status

Longitude 11820.00 state

Name

NV

\begin{tabular}{|c|c|c|c|c|}
\hline $\begin{array}{l}\text { Unit } \\
\text { Type }\end{array}$ & $\begin{array}{l}\text { Plant } \\
\text { Type }\end{array}$ & $\begin{array}{l}\text { Project } \\
\text { status }\end{array}$ & $\begin{array}{l}\text { Dam } \\
\text { status }\end{array}$ & $\begin{array}{lr}\text { Latitude } & 4155.00 \\
\text { Longitude } & 11820.00\end{array}$ \\
\hline C & ROR & $X X$ & $\mathrm{U}$ & \\
\hline
\end{tabular}

Factor

Exist Prob

Factor

Exist Prob

Wild/Scenic Protection

wild/Scenic Tributary or

Upstream/Downstream

0.90 Wildife Value

Threatened/Endangered Fish

Threatened/Endangered Wildlife

0.90 Federal Land Code 103

0.90 Federal Land Code 104

0.90 Federal Land Code 105

0.90 Federal Land Code 106

0.90 Federal Land Code 107

0.90 Federal Land Code 108

0.90 Federal Land Code 198
PESF Annual Energy Rating (MWh)

162.90

Cultural value

0.90

Geologic Value

other Value

Recreation Value

Scenic Value

0.90

0.90

0.90

0.90

0.90

0.90

0.90

0.90

0.90

0.90 
R E S O U R C E D A T A B A S E I I S T I N G

DATE: $08 / 19 / 97$

PAGE NO: 106

FERC

Number

Plant Name

Stream

State

NVHO038 GRANITE PLANT

GRANITE CREEK

Name

NV

county Name

River Basin

HUMBOLDT

BLACK ROCK DESERT BASIN

Class Owner Name

F U.S.D.I. BUREAU OF IAND MANAGEMENT

\begin{tabular}{|c|c|c|c|c|c|c|}
\hline \multirow[t]{2}{*}{$\begin{array}{l}\text { Name } P \\
\text { Rating }\end{array}$} & $\begin{array}{l}\text { late } \\
(\mathrm{KW})\end{array}$ & PESF & PESF*KW & $\begin{array}{l}\text { Annu } \\
\text { Rati }\end{array}$ & $\begin{array}{l}\text { Energy } \\
\text { (MWh) }\end{array}$ & $\begin{array}{l}\text { PESF Annual } \\
\text { Energy Rating (MWh) }\end{array}$ \\
\hline & 29.00 & 0.90 & 26.10 & & 120.00 & 108 \\
\hline $\begin{array}{l}\text { Unit } \\
\text { Type }\end{array}$ & $\begin{array}{l}\text { Pla } \\
\text { Tyl }\end{array}$ & & $\begin{array}{l}\text { Project } \\
\text { Status }\end{array}$ & $\begin{array}{l}\text { Dam } \\
\text { status }\end{array}$ & $\begin{array}{l}\text { Latitude } \\
\text { Longitude }\end{array}$ & $\begin{array}{r}4156.00 \\
11815.00\end{array}$ \\
\hline C & & & $X X$ & $\mathrm{U}$ & & \\
\hline
\end{tabular}

Factor

Exist Prob

Factor

Exist Prob

Wild/Scenic Protection

wild/Scenic Tributary or

0.90 Wildlife Value

Threatened/Endangered Fish

Upstream/Downstream

wild/Scenic Location

Threatened/Endangered Wildlife

0.90

0.90

0.90 Federal Land Code 103

0.90

0.90 Federal Land Code 104

0.90

0.90 Federal Land Code 105

0.90

Fish Presence value

0.90 Federal Land Code 106

0.90

0.90 Federal Land Code 107

0.90

Historic Value

0.90 Federal Land Code 108

0.90

other Value

Recreation Value

0.90 Federal Land Code 198

0.90

0.90

0.90

Scenic Value 
DATE: $08 / 19 / 97$

PAGE NO: 107

FERC

Number

Plant Name

stream

state

Name

NVH0039 MCDERMITT \#1

EAST QUINN RIVER

NV

county Name

HUMBOLDT
River Basin

BLACK ROCK DESERT BASIN $\begin{array}{cc}\text { Class } & \text { Owner Name } \\ \text { C } & \text { U.S.D.I. BUREAU OF INDIAN AFFAIRS }\end{array}$

Name Plate

Rating (KW)

PESF

Annual Energy

PESF*KW Rating (MWh)

PESF Annual

136.00

$0.50 \quad 68.00$

489.00

244.50

\begin{tabular}{|c|c|c|c|c|c|}
\hline $\begin{array}{l}\text { Unit } \\
\text { Type }\end{array}$ & $\begin{array}{l}\text { Plant } \\
\text { Type }\end{array}$ & $\begin{array}{l}\text { Project } \\
\text { status }\end{array}$ & $\begin{array}{l}\text { Dam } \\
\text { status }\end{array}$ & $\begin{array}{l}\text { Latitude } \\
\text { Longitude }\end{array}$ & $\begin{array}{r}4158.00 \\
11735.00\end{array}$ \\
\hline C & STG & $\mathrm{XX}$ & $\mathrm{U}$ & & \\
\hline
\end{tabular}

Factor

Exist Prob

Factor

Exist Prob

Wild/Scenic Protection

Wild/Scenic Tributary or

Upstream/Downstream

wild/Scenic Location

Cultural value

Fish Presence Value

Geologic Value

Historic value

other value

Recreation value

Scenic Value

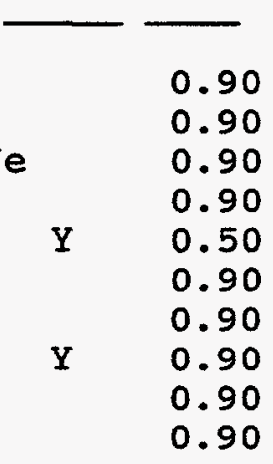


RESOURCE DA TABAS E I I S T IN G

DATE: $08 / 19 / 97$

PAGE NO: 108

FERC

Number

Plant Name

stream

State

NVH0040 MCDERMITT \#2

MCDERMITT CREEK

Name

NV

County Name

River Basin

HUMBOLDT

BLACK ROCK DESERT BASIN

Class Owner Name

C UNKNOWN

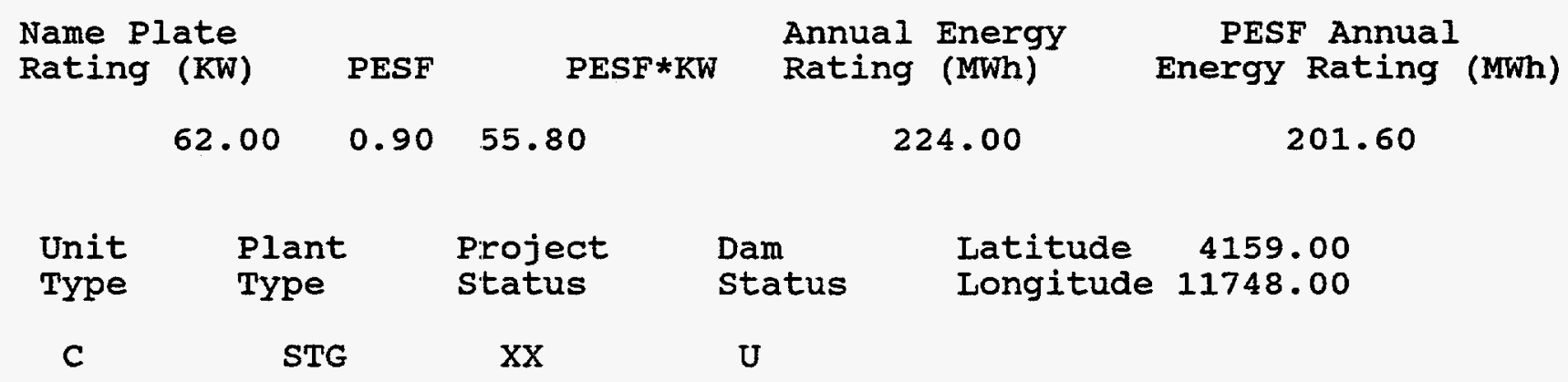

Factor

Exist Prob

Factor

Exist Prob

Wild/Scenic Protection

Wild/Scenic Tributary or

0.90 Wildlife Value

Threatened/Endangered Fish

Upstream/Downstream Threatened/Endangered Wildlife

0.90

0.90

0.90

0.90 Federal Land code 103

0.90

0.90 Federal Land Code 104

0.90

0.90 Federal Land Code 105

0.90

0.90 Federal Land Code 106

0.90

0.90 Federal Land Code 107

0.90

Historic Value

0.90

Federal Land Code 108

0.90

Recreation value

0.90

Federal Land Code 198

0.90

Scenic Value

0.90 
DATE: $08 / 19 / 97$

FERC

Number

Plant Name

NVH0042 PARADISE MILL

County Name

HUMBOLDT

Class Owner Name

C UNKNOWN

Name Plate

Rating (KW)

PESF

Annual Energy

126.00

$0.75 \quad 94.50$

PESF*KW

Rating (MWh)

455.00

Latitude $\quad 4132.00$

Unit Plant

Type Type

C

ROR

Factor

Exist Prob

Factor

Exist Prob

Wild/Scenic Protection

wild/Scenic Tributary or

Upstream/Downstream

wild/Scenic Location

Cultural value

Fish Presence Value

Geologic Value

Historic Value

other Value

Recreation Value

Scenic Value
PAGE NO: 109

state

Name

NV
PESF Annual Energy Rating (MWh)

341.25

$\begin{array}{lllr}\text { Project } & \text { Dam } & \text { Latitude } & 4132.00 \\ \text { Status } & \text { Status } & \text { Longitude } & 11725.00\end{array}$

$\mathrm{XX}$

W

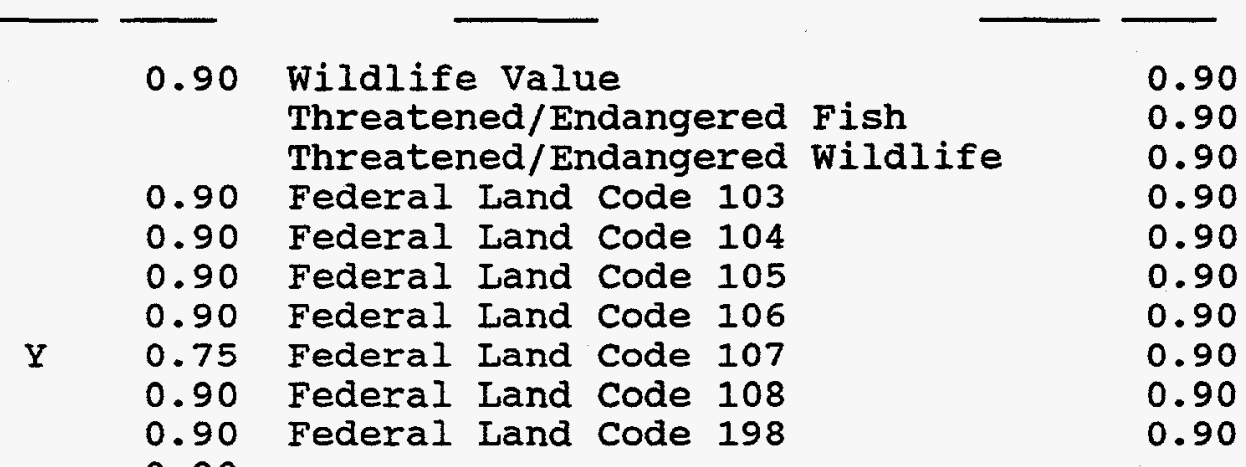



R E S O U R C E
D A T A B A E
I I S T N G

DATE : $08 / 19 / 97$

PAGE NO: 110

FERC

Number

Plant Name

Stream

State

NVHO043 PARADISE \#1

BIG COTTONWOOD CREEK

Name

County Name

HUMBOLDT

River Basin

HUMBOLDT RIVER BASIN

class Owner Name

R UNKNOWN

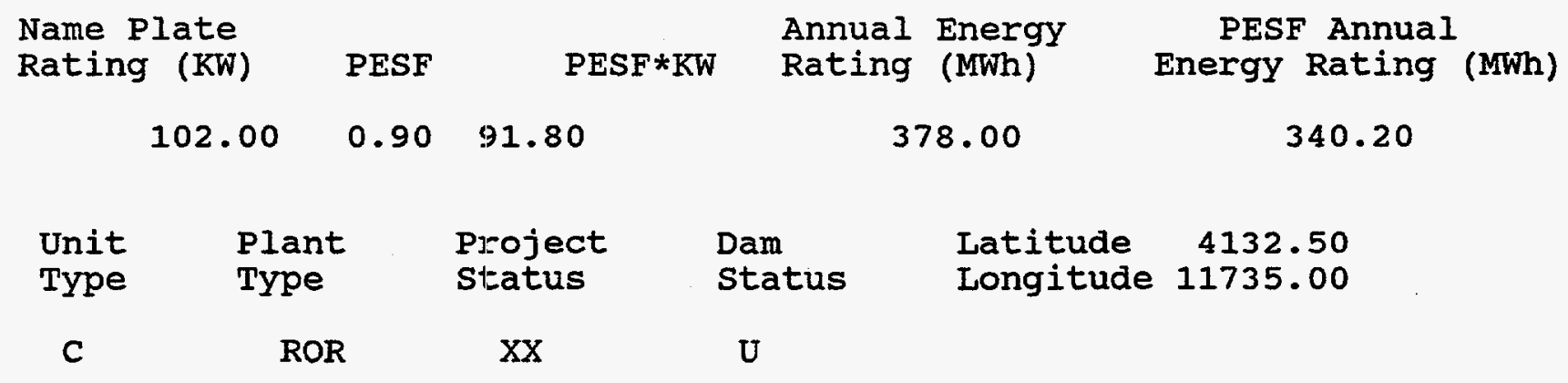

Factor

Exist Prob

Factor

Exist Prob

wild/Scenic Protection

wild/Scenic Tributary or

0.90 Wildlife Value

Threatened/Endangered Fish

NV

Upstream/Downstream

wild/Scenic Location

Cultural value

Fish Presence value

Geologic Value

Historic Value

other Value

Recreation value Threatened/Endangered Wildlife

0.90

$\begin{array}{ll}0.90 & \text { Federal Land Code } 103 \\ 0.90 & \text { Federal Land Code } 104\end{array}$

0.90 Federal Land Code 105

0.90 Federal Land Code 106

0.90 Federal Land Code 107

0.90 Federal Land Code 108

0.90 Federal Land Code 198

0.90

0.90

0.90

0.90

0.90

0.90

0.90

0.90

0.90

0.90

Scenic Value 
FERC

Number

NVH0044 PARADISE \#2

County Name

HUMBOLDT

Class Owner Name

C UNKNOWN

Name Plate

Rating (KW)

183.00
Annual Energy Rating (MWh)

672.00
State

Name

NV
River Basin

HUMBOLDT RIVER BASIN

\begin{tabular}{|c|c|c|c|c|}
\hline $\begin{array}{l}\text { Unit } \\
\text { Type }\end{array}$ & $\begin{array}{l}\text { Plant } \\
\text { Type }\end{array}$ & $\begin{array}{l}\text { Project } \\
\text { Status }\end{array}$ & $\begin{array}{l}\text { Dam } \\
\text { status }\end{array}$ & $\begin{array}{lr}\text { Latitude } & 4134.00 \\
\text { Longitude } & 11731.00\end{array}$ \\
\hline C & ROR & $x X$ & $\mathbf{U}$ & \\
\hline
\end{tabular}

Factor

Wild/Scenic Protection

Wild/Scenic Tributary or

Upstream/Downstream

wild/Scenic Location

Cultural Value

Fish Presence Value

Geologic Value

Historic Value

other value

Recreation Value

Scenic Value
Exist Prob

0.90

Wildlife Value Threatened/Endangered Fish Threatened/Endangered Wildife

0.90 Federal Land Code 103

0.90 Federal Land Code 104

0.90 Federal Land Code 105

0.90 Federal Land Code 106

0.90 Federal Land Code 107

0.90 Federal Land Code 108

0.90 Federal Land Code 198
PESF Annual Energy Rating (MWh)

336

Exist Prob

$\begin{array}{ll} & \\ & \\ & 0.90 \\ & 0.90 \\ & 0.90 \\ & 0.90 \\ \mathrm{Y} & 0.50 \\ & 0.90 \\ & 0.90 \\ & 0.90 \\ & 0.90 \\ & 0.90\end{array}$



RESOU R C E
D A T A B A S E
I I S T N G

DATE : 08/19/97

PAGE NO: 112

FERC

Number

Plant Name

stream

State

Name

NVH0045 GOLCONDA PLANT

POLE CREEK

NV

County Name

River Basin

HUMBOLDT

HUMBOLDT RIVER BASIN

Class Owner Name

C UNKNOWN

Name Plate

Rating (KW)

PESF

Annual Energy

Rating (MWh)

PESF Annual

73.00

$0.90 \quad 65.70$

280.00

Energy Rating (MWh)

Unit Plant

Type Type

Project

Dam Status

Status

Latitude $\quad 4055.00$

C

ROR

$\mathrm{xx}$

U

Factor

Exist Prob

Factor

Exist Prob

Wild/Scenic Protection

Wild/Scenic Tributary or Upstream/Downstream wild/Scenic Location

Cultural value

Fish Presence Value

Geologic Value

Historic Value

other Value

Recreation Value

Scenic value

0.90 Wildife Value

Threatened/Endangered Fish

252

Threatened/Endangered Wildlife

0.90

0.90

0.90 Federal Land Code 103

0.90 Federal Land Code 104

0.90

Federal Land Code 105

0.90

0.90

Federal Land Code 106

0.90

0.90

Federal Land Code 107

0.90

0.90

Federal Land Code 108

0.90

0.90

Federal Land Code 198

0.90

0.90

0.90

0.90

0.90 
DATE: $08 / 19 / 97$

PAGE NO: 113

FERC

Number Plant Name

stream

State

NVHO046 ROCK CREEK DAM

ROCK CREEK

Name

NVHO046 ROCK CREEK DAM

county Name

River Basin

LANDER

HUMBOLDT RIVER BASIN

\section{Class Owner Name}

C IANDER COUNTY

Name Plate

Rating ( $\mathrm{KW}$ )

137.00
PESF

$0.25 \quad 34.25$
Annual Energy

Rating (MWh)

493.00
NV

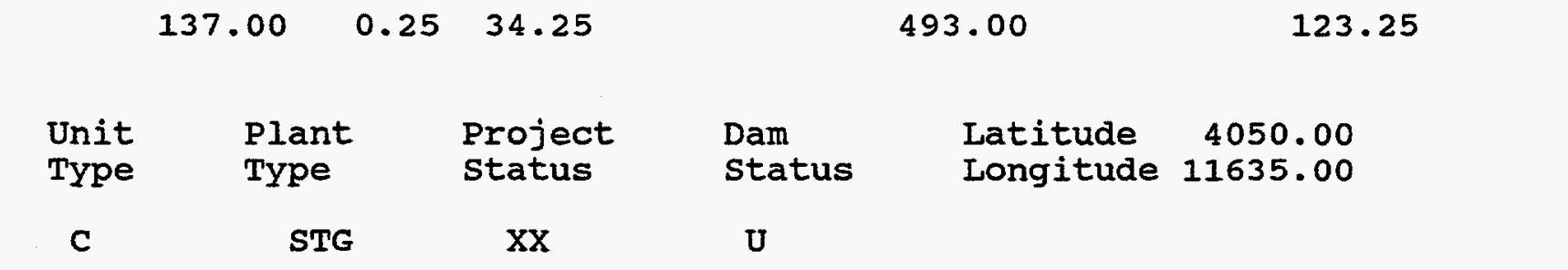

Factor

Exist Prob

wild/Scenic Protection

wild/Scenic Tributary or Upstream/Downstream wild/Scenic Location Cultural Value

Fish Presence Value

Geologic Value

Historic Value

other Value

Recreation value

Scenic Value
Factor

Exist Prob

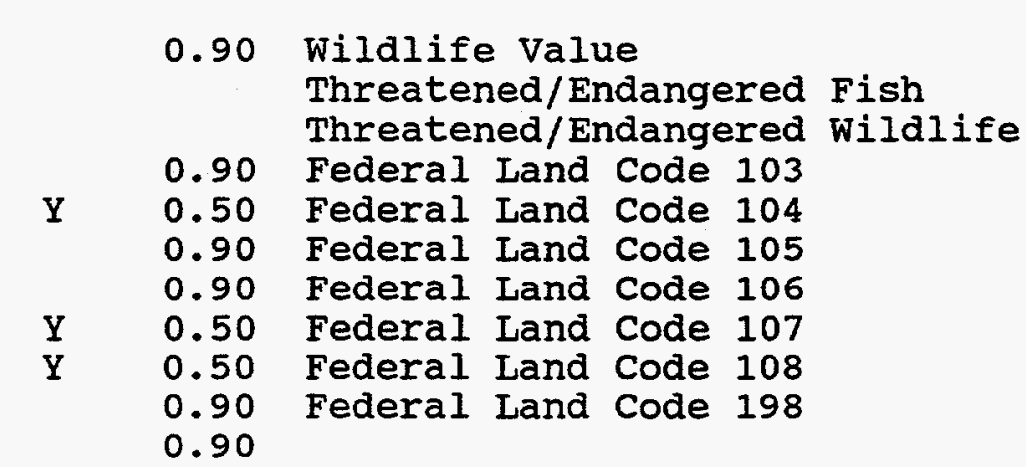

0.90

0.90

0.90

0.90

0.90

0.90

0.90

0.90

0.90

0.90 
R E S O U R C E

DATE : $08 / 19 / 97$
D A T A B A S E

FERC

Number

Plant Name

NVHO048 URSINE PLANT

County Name

LINCOLN

class

Owner Name

C IJNKNOWN
PAGE NO: 114

$\begin{array}{cc}\text { Stream } & \begin{array}{c}\text { State } \\ \text { Name }\end{array} \\ \text { MEADOW VALLEY WASH } & \mathrm{NV} \\ \text { River Basin } & \\ \text { COLORADO RIVER BASIN } & \end{array}$

State

NV

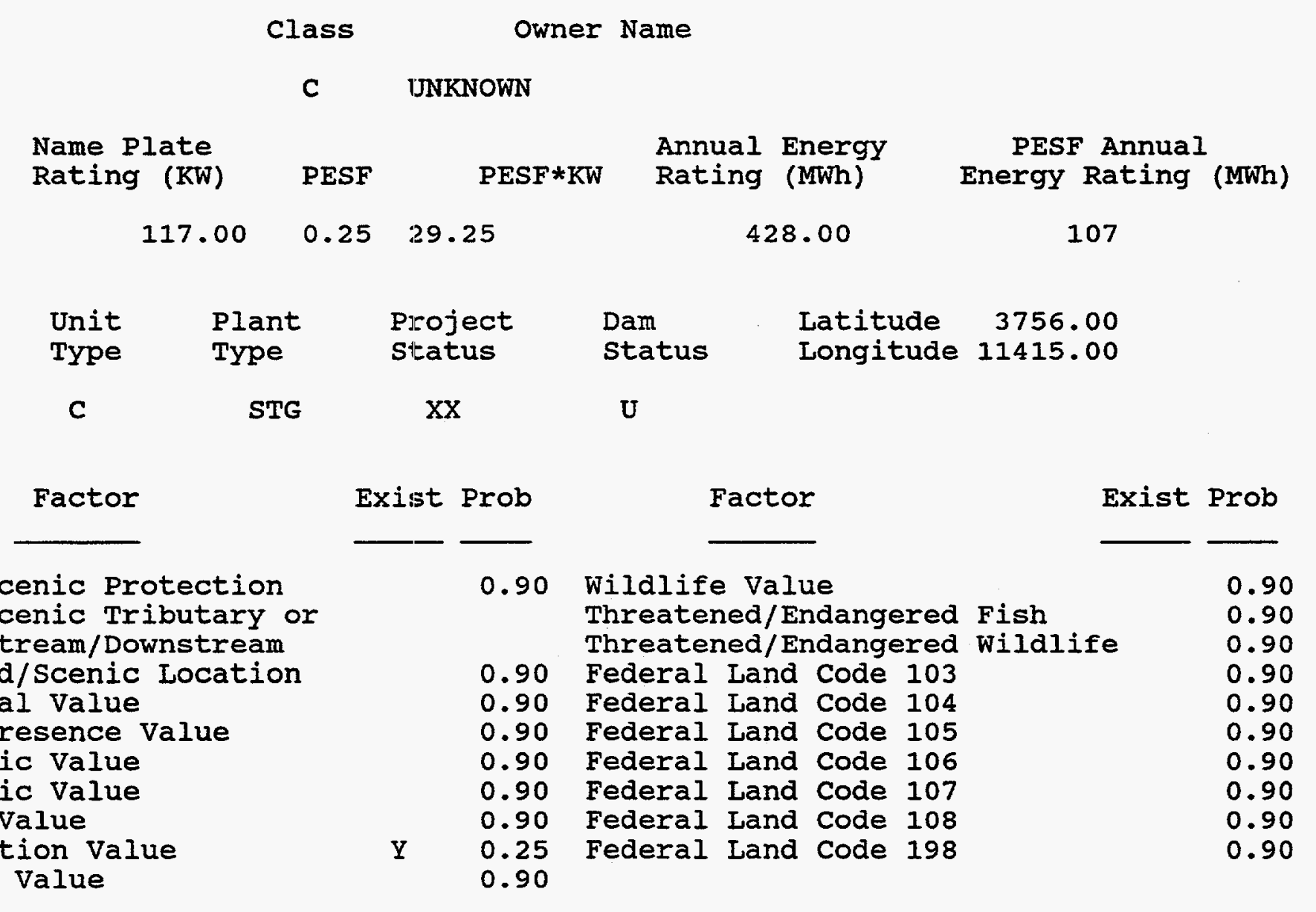

Wild/Scenic Protection

wild/scenic Tributary or

Upstream/Downstream wild/Scenic Location

Cultural value

Fish Presence Value

Geologic Value

Historic Value

other value

Recreation Value

Scenic Value
Exist Prob

0.90

Threatened/Endan

Threatened/Endangered Wildife

Federal Land Code 103

0.90 Federal Land Code 104

0.90 Federal Land Code 105

0.90 Federal Land Code 106

0.90 Federal Land Code 107

0.90 Federal Land Code 108

0.90
0.90

0.90

0.90

0.90

0.90

0.90

0.90 
DATE: $08 / 19 / 97$

FERC

Number

Plant Name

NVHOO49 EAST FORK POWER PLANT

County Name

IYON
PAGE NO: 115

State

Name

EAST FORK WALKER RIVER

NV

Class Owner Name

R UNKNOWN

Name Plate

Rating (KW)

108.00

PESF

Annual Energy

PESF*KW

Rating (MWh)

417.00

$0.50 \quad 54.00$

Plant

Type

STG

Project

status

$\mathrm{XX}$

Exist Prob

Factor

Latitude 3850.00

Dam

Status

Longitude 11905.00
PESF Annual Energy Rating (MWh)

208.50
Factor

Wild/Scenic Protection

wild/Scenic Tributary or Upstream/Downstream wild/Scenic Location cultural value Fish Presence Value Geologic Value Historic Value other Value Recreation Value Scenic Value
Exist Prob

0.90

wildlife value

Threatened/Endangered Fish

Threatened/Endangered Wildlife

0.90 Federal Land Code 103

0.90 Federal Land Code 104

0.90 Federal Land Code 105

0.90 Federal Land Code 106

0.90 Federal Land Code 107

$Y \quad 0.50$ Federal Land Code 108

0.90 Federal Land Code 198

0.90
Exist Prob

0.90

0.90

0.90

0.90

0.90

0.90

0.90

0.90

0.90

0.90 
RE SOURCE D A T A B A S E L I S T IN G

DATE: $08 / 19 / 97$

PAGE NO: 116

FERC

Number

Plant Name

Stream

State

NVH0051 YOMBA PLANT

REESE RIVER

Name

NV

County Name

River Basin

NYE

HUMBOLDT RIVER BASIN

Class

Owner Name

F U.S.D.I. BUREAU OF INDIAN AFFAIRS

Name Plate

Annual Energy

Annual Energy
Rating (MWh)

PESF Annual

Rating (KW)

PESF

324.00

Energy Rating (MWh)

$89.00 \quad 0.50 \quad 44.50$

Project

status

Dam

status

Latitude $\quad 3855.00$

Type

Type

$\mathrm{XX}$

U

Factor

Exist Prob

Factor

Wild/Scenic Protection

wild/Scenic Tributary or

0.90 Wildife Value

Threatened/Endangered Fish

Upstream/Downstream

wild/Scenic Location

Cultural value

Fish Presence Value

Geologic Value

Historic Value

other Value

Recreation value

Scenic value
Threatened/Endangered Wildlife

0.90 Federal Land Code 103

0.90 Federal Land Code 104

0.90 Federal Land Code 105

0.90 Federal Land Code 106

0.90 Federal Land Code 107

0.90 Federal Land Code 108

0.90 Federal Land Code 198

0.90
Exist Prob

162

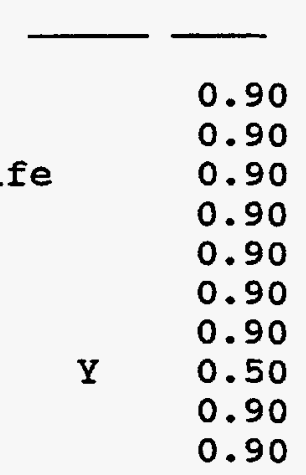


DATE: $08 / 19 / 97$

FERC

Number

Plant Name

NVH0052 ARC DOME \#1

County Name

NYE
PAGE NO: 117

State

Name

SOUTH TWIN RIVER

NV

\section{Class Owner Name}

F U.S.D.A. FOREST SERVICE

Name Plate

Rating ( $\mathrm{KW}$ )

Annual Energy

PESF

Rating (MWh)

PESF Annual

162.00

$0.50 \quad 81.00$

608.00

304

$\begin{array}{cc}\text { Unit } & \text { Plant } \\ \text { Type } & \text { Type } \\ \text { C } & \text { STG }\end{array}$

Project

status

$\mathrm{XX}$

$\begin{array}{llr}\text { Dam } & \text { Latitude } & 3853.00 \\ \text { Status } & \text { Longitude } & 11714.00\end{array}$

U
Factor

Wild/Scenic Protection

Wild/Scenic Tributary or

Upstream/Downstream

wild/Scenic Location

Cultural Value

Fish Presence Value

Geologic Value

Historic Value

other Value

Recreation value

Scenic Value
Exist Prob

0.90

Wildife Value

Threatened/Endangered Fish

Threatened/Endangered Wildlife

0.90 Federal Land Code 103

0.90 Federal Land Code 104

0.90 Federal Land Code 105

0.90 Federal Land Code 106

0.90 Federal Land Code 107

0.90 Federal Land Code 108

0.90 Federal Land Code 198
Exist Prob

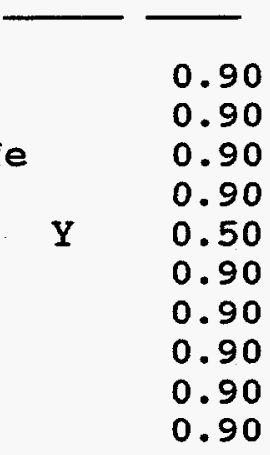


FERC

Number

NVHO053 CURRANT PLANT

County Name

NYE
Plant Name

Class

R UNKNOWN
State

Name

CURRANT CREEK

NV

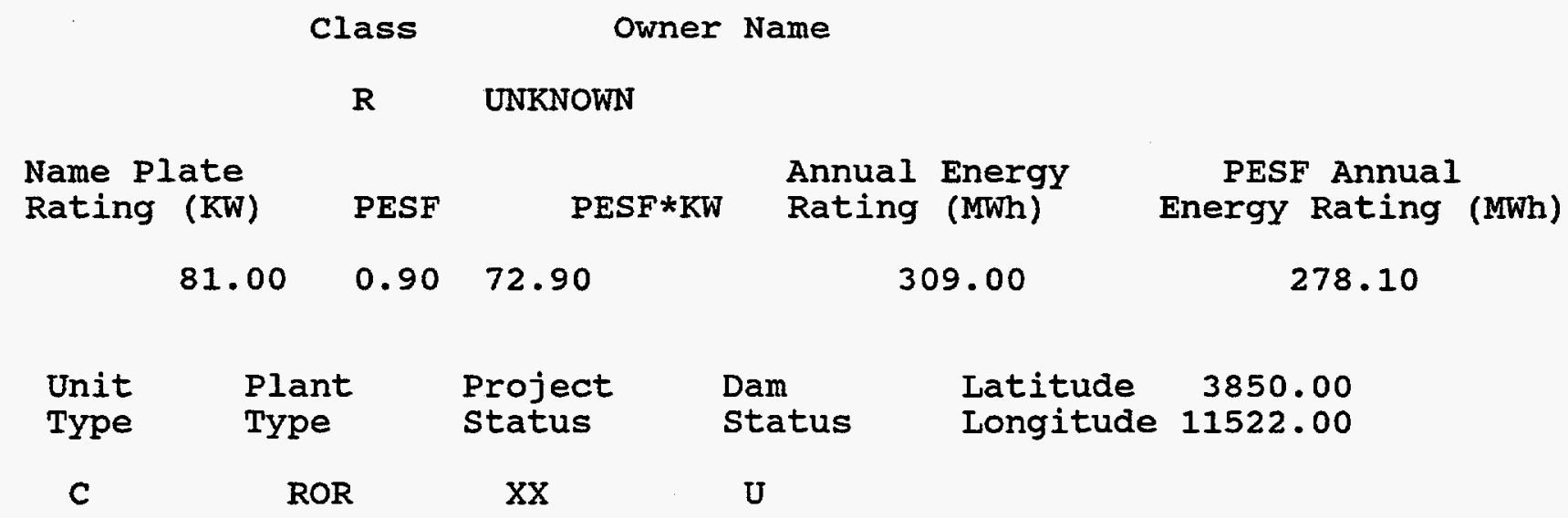

Factor

Exist Prob

Factor

Exist Prob

Wild/Scenic Protection

Wild/Scenic Tributary or

0.90 Wildlife value

Threatened/Endangered Fish

Upstream/Downstream

wild/Scenic Location

Cultural value

Fish Presence Value

Geologic Value

Historic Value

other Value

Recreation Value

Scenic Value
River Basin

RAILROAD BASIN
PESF Annual

$$
278.10
$$

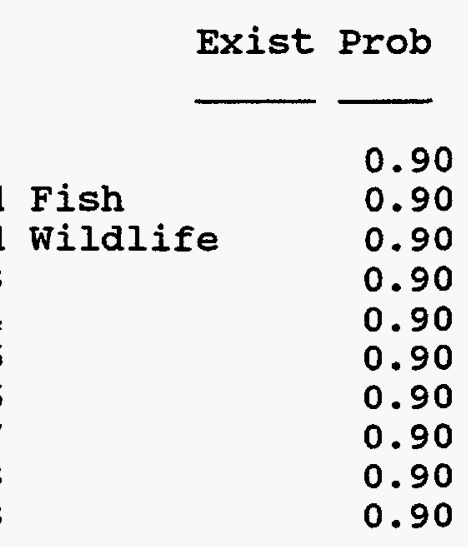


DATE: $08 / 19 / 97$

FERC

Number

NVHO055 WHITE'S PLANT

County Name

WASHOE
PAGE NO: 119

State

Name

WHITE'S CREEK

NV

River Basin

TRUCKEE RIVER BASIN

Class Owner Name

R UNKNOWN

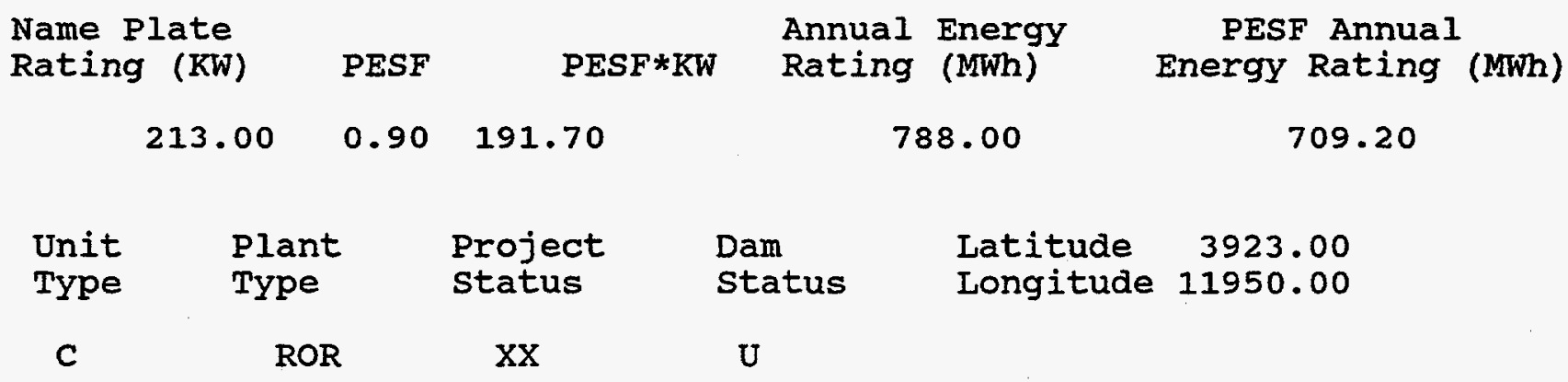

Factor

Exist Prob

Factor

Exist Prob

Wild/Scenic Protection

wild/Scenic Tributary or

0.90 Wildlife Value

Threatened/Endangered Fish

Upstream/Downstream

wild/Scenic Location

Threatened/Endangered Wildlife

0.90 Federal Land Code 103

0.90 Federal Land code 104

$\longrightarrow$

0.90 Federal Land Code 105

0.90 Federal Land Code 106

0.90 Federal Land Code 107

0.90

0.90

0.90

Cultural value

Fish Presence Value

0.90 Federal Land Code 108

0.90

0.90

Geologic Value

0.90 Federal Irand Code 198

0.90

0.90

0.90

other value

Recreation Value

0.90

0.90

0.90 
R E S O UR C E D A T A B A S E I I S T I N G

DATE: $08 / 19 / 97$

PAGE NO: 120

FERC

Number

Plant Name

stream

State

NVH0063 GOSHUTE PLANT

SPRING CREEK

Name

County Name

River Basin

WHITE PINE

GREAT SAIT IAKE BASIN

class Owner Name

F U.S.D.I. BUREAU OF INDIAN AFFAIRS

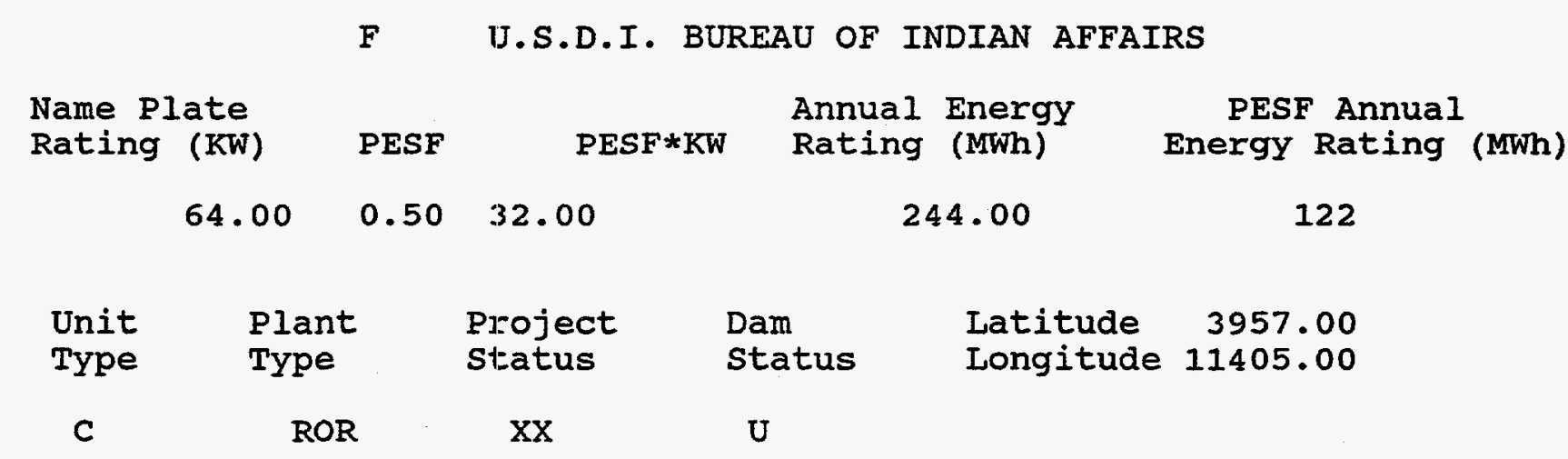

Factor

wild/scenic Protection

Wild/Scenic Tributary or Upstream/Downstream wild/Scenic Location Cultural value Fish Presence Value Geologic Value Historic Value other value Recreation value Scenic Value
Exist Prob

0.90 Wildlife Value
Threatened/Endan Threatened/Endangered Fish Threatened/Endangered Wildlife

0.90 Federal Land Code 103

0.90 Federal Land Code 104

0.90 Federal Land Code 105

0.90 Federal Land Code 106

0.90 Federal Land Code 107

0.90 Federal Land Code 108

0.90 Federal Land Code 198

0.90
Exist Prob

0.90

0.90

0.90

0.90

0.90

0.90

0.90

$Y$

0.90

0.90 
DATE: $08 / 19 / 97$

FERC

Number

Plant Name

NVH0065 BAKER PLANT

County Name

WHITE PINE

Class

Owner Name

R UNKNOWN

Name Plate Rating (KW)

PESF

Annual Energy

PESF*KW

Rating (MWh)

592.00

PAGE NO: 121

BAKER CREEK

River Basin

GREAT SALT LAKE BASIN

$$
161.00 \quad 0.90 \quad 144.90
$$

State

Name

NV
Project status

Type

c Type

ROR

$\mathrm{XX}$
PESF Annual Energy Rating (MWh)

532.80
Factor

Wild/Scenic Protection

wild/Scenic Tributary or

Upstream/Downstream

wild/Scenic Location

Cultural Value

Fish Presence Value

Geologic Value

Historic Value

other Value

Recreation Value

Scenic Value
Dam
status

U
Exist Prob

Factor

Latitude $\quad 3859.00$

Longitude 11413.00
0.90 Wildlife Value
Threatened/Endangered Fish Threatened/Endangered Wildlife
0.90
Federal Land Code 103
0.90 Federal Land Code 104
0.90 Federal Land Code 105
0.90 Federal Land Code 106
0.90 Federal Land Code 107
0.90 Federal Land Code 108
0.90 Federal Land Code 198
0.90

Exist Prob

Exist Prob

0.90

0.90

0.90

0.90

0.90

0.90

0.90

0.90

0.90

0.90 
R E SOU R C E D A TA B A S E I I S T I N G

DATE: $08 / 19 / 97$

PAGE NO: 122

FERC

Number

Plant Name

Stream

State

Name

NVH0068 MOTT CANYON PLANT

MOTT CREEK

NV

county Name

DOUGLAS

Class

R INNKNOWN

Name Plate

Rating ( $\mathrm{KW}$ )

PESF

$\mathrm{PESF} * \mathrm{KW}$

Annual Energy

Rating (MWh)

163.00

$0.90 \quad 146.70$

588.00

Dam

Status

Latitude $\quad 3856.00$

$\begin{array}{ll}\text { Unit } & \text { Plant } \\ \text { Type } & \text { Type }\end{array}$

Project

status

C

ROR

$\mathrm{xx}$

U
PESF Annual Energy Rating (MWh)

529.20
Factor

Wild/Scenic Protection

Wild/Scenic Tributary or Upstream/ Downstream wild/Scenic Location

Cultural Value

Fish Presence Value

Geologic Value

Historic Value

other Value

Recreation Value

Scenic Value
Exist Prob

0.90

Wildlife value Threatened/Endangered Fish Threatened/Endangered Wildlife

0.90 Federal Land code 103

0.90 Federal Land Code 104

0.90 Federal Land Code 105

0.90 Federal Land Code 106

0.90 Federal Land Code 107

0.90 Federal Land Code 108

0.90 Federal Land Code 198

0.90

Exist Prob

0.90

0.90

0.90

0.90

0.90

0.90

0.90

0.90

0.90

0.90 
R E S O U R C E

DATE: $08 / 19 / 97$

FERC

Number

Plant Name

NVH0069 MOUNT SEIGEL PLANT

county Name

DOUGLAS
D A T A B A S E

L I S T I G

\section{PAGE NO: 123}

State

Name

PINE NUT CREEK

NV

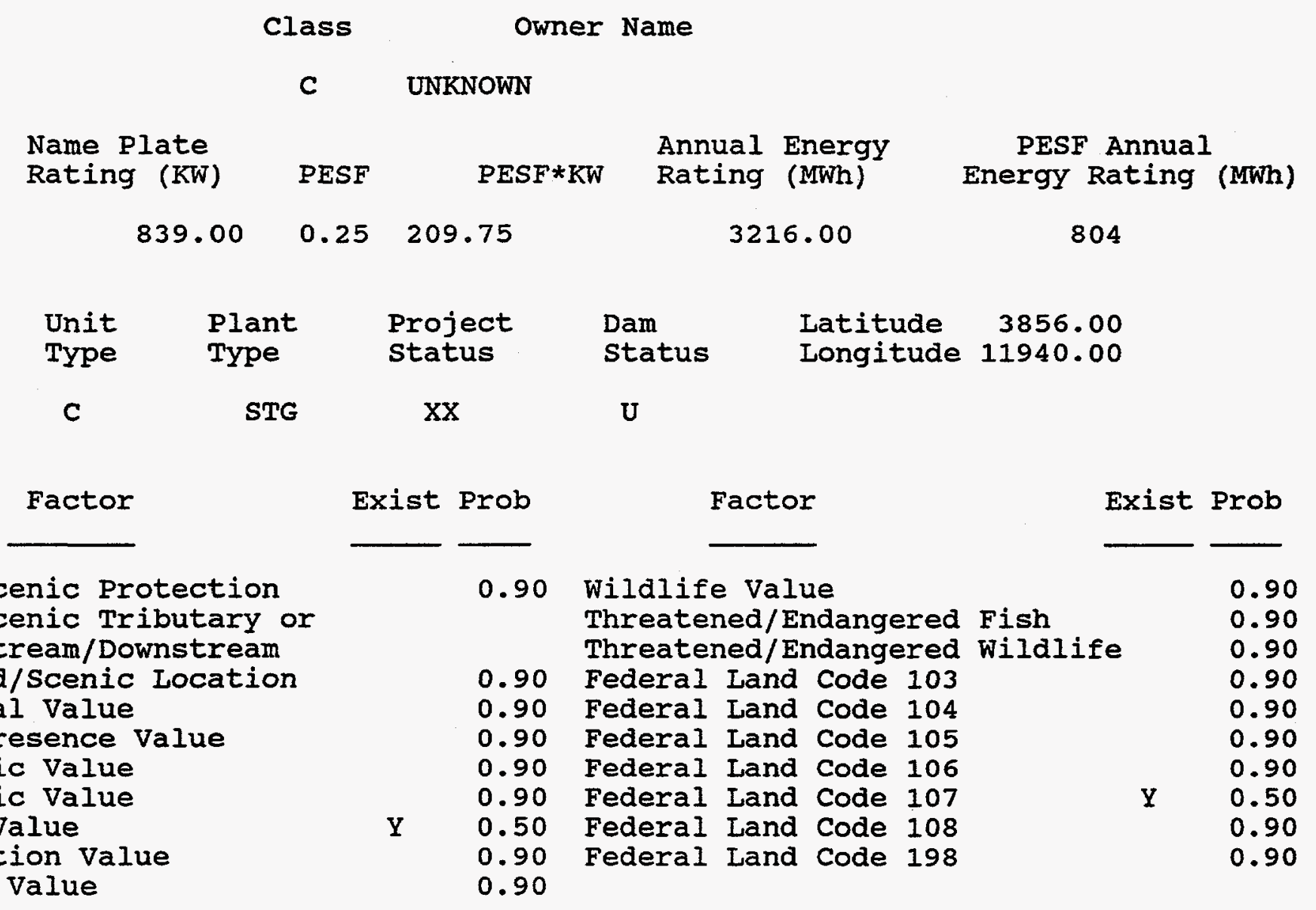

Wild/Scenic Protection

Wild/Scenic Tributary or Upstream/Downstream wild/Scenic Location Cultural value Fish Presence Value Geologic Value Historic Value other Value Recreation value Scenic Value
River Basin

CARSON RIVER BASIN 
R E S O U R C E

DATE: $08 / 19 / 97$
D A T A B A E I I S T I N G

PAGE NO: 124

FERC

Number

Plant Name

NVH0071 B.C. PLANT

County Name

ELKO

River Basin
SNAKE RIVER BASIN
COON CREER

River Basin
KKE RIVER BASIN
State

Name

NV

Class Owner Name

F U.S.D.A. FOREST SERVICE
Name Plate
Rating ( $\mathrm{KW}$ )
PESF
PESF*KW
Annual Energy
Rating (MWh)

102.00

$0.25 \quad 25.50$

387.00
Plant

Type

STG

Unit
Type

C

Factor

Wild/Scenic Protection

wild/Scenic Tributary or Upstream/Downstream wild/Scenic Location

Cultural Value

Fish Presence Value

Geologic Value

Historic Value

other Value

Recreation value

Scenic Value
Exist Prob

Project status

$\mathrm{XX}$ $\begin{array}{llr}\text { Dam } & \text { Latitude } & 4149.00 \\ \text { Status } & \text { Longitude } & 11535.00\end{array}$

$\begin{array}{llr}\text { Dam } & \text { Latitude } & 4149.00 \\ \text { Status } & \text { Longitude } & 11535.00\end{array}$

U
PESF Annual Energy Rating (MWh)

96.75

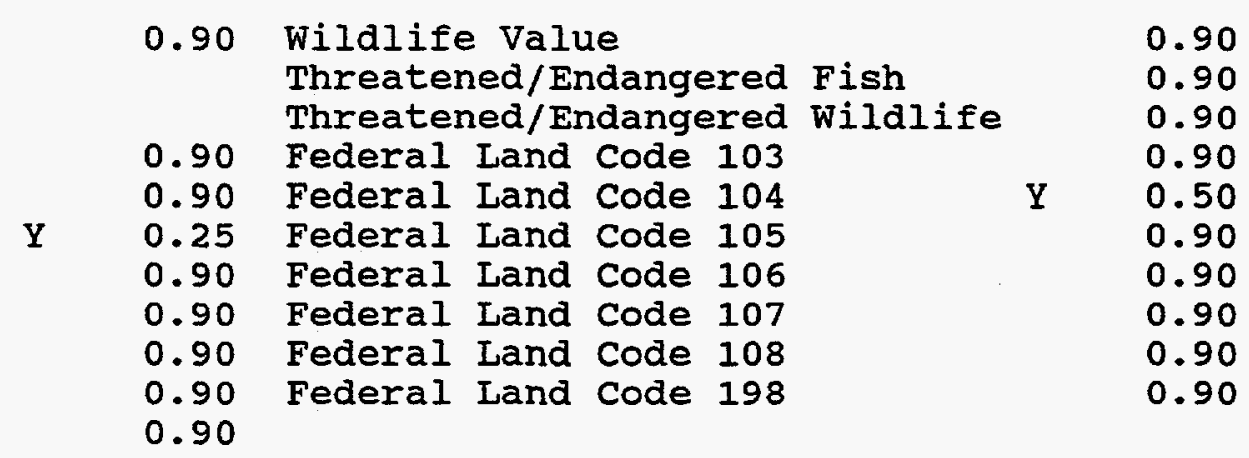


FERC

Number

Plant Name

NVH0072 COPPER MOUNTAIN PLANT

county Name
EIKO

\author{
County Name
}

ELKO

SNAKE RIVER BASIN

\section{Class Owner Name}

C UNKNOWN
State

Name

NV

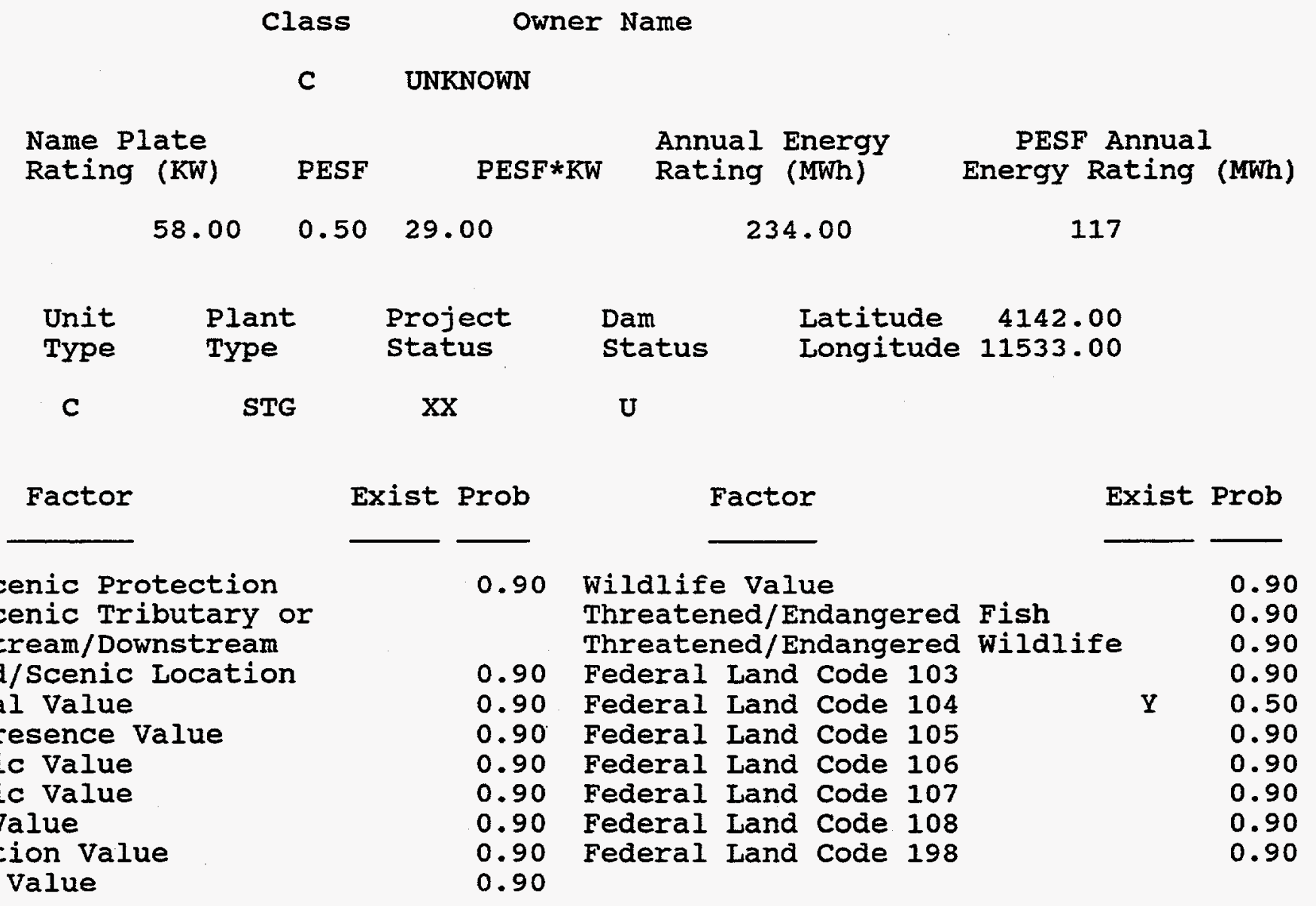

Wild/scenic Protection

wild/Scenic Tributary or Upstream/Downstream wild/Scenic Location Cultural Value Fish Presence Value Geologic Value Historic Value other Value Recreation Value Scenic Value
COPPER CREER

River Basin

SNAKE RIVER BASIN 

R E S O U R C E
D A T A B A S E
I I T I N G

DATE: $08 / 19 / 97$

PAGE NO: 126

FERC

Number

Plant Name

stream

State

NVH0073 BIG BUCK PLANT

BUCK CREEK

Name

NV

county Name

River Basin

ELKO

SNAKE RIVER BASIN

class Owner Name

C UNKNOWN

Name Plate

Rating ( $\mathrm{KW}$ )

PESF

Annual Energy

PESF Annual

$197.00 \quad 0.10 \quad 19.70$

724.00

Energy Rating (MWh)

PESF*KW Rating (MWh)

72.40

\begin{tabular}{|c|c|c|c|c|}
\hline $\begin{array}{l}\text { Unit } \\
\text { Type }\end{array}$ & $\begin{array}{l}\text { Plant } \\
\text { Type }\end{array}$ & $\begin{array}{l}\text { Project } \\
\text { status }\end{array}$ & $\begin{array}{l}\text { Dam } \\
\text { status }\end{array}$ & $\begin{array}{lr}\text { Latitude } & 4158.00 \\
\text { Longitude } & 11526.00\end{array}$ \\
\hline C & ROR & $x x$ & $\mathrm{U}$ & \\
\hline
\end{tabular}

Factor

Wild/Scenic Protection

Wild/Scenic Tributary or Upstream/Downstream wild/Scenic Location Cultural Value

Fish Presence Value

Geologic Value

Historic Value

other Value

Recreation Value

Scenic Value
Exist Prob

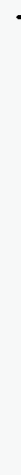

Exist Prob

Factor

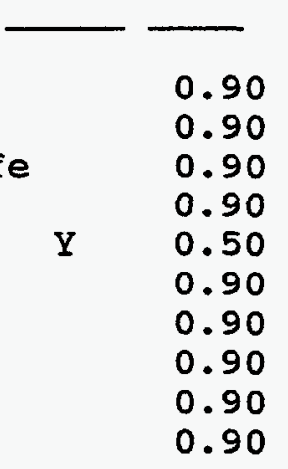


DATE: $08 / 19 / 97$

PAGE NO: 127

FERC

Number

NVHO074 DAVE POWER PLANT

County Name

ELKO
Plant Name

Owner Name

C UNKNOWN

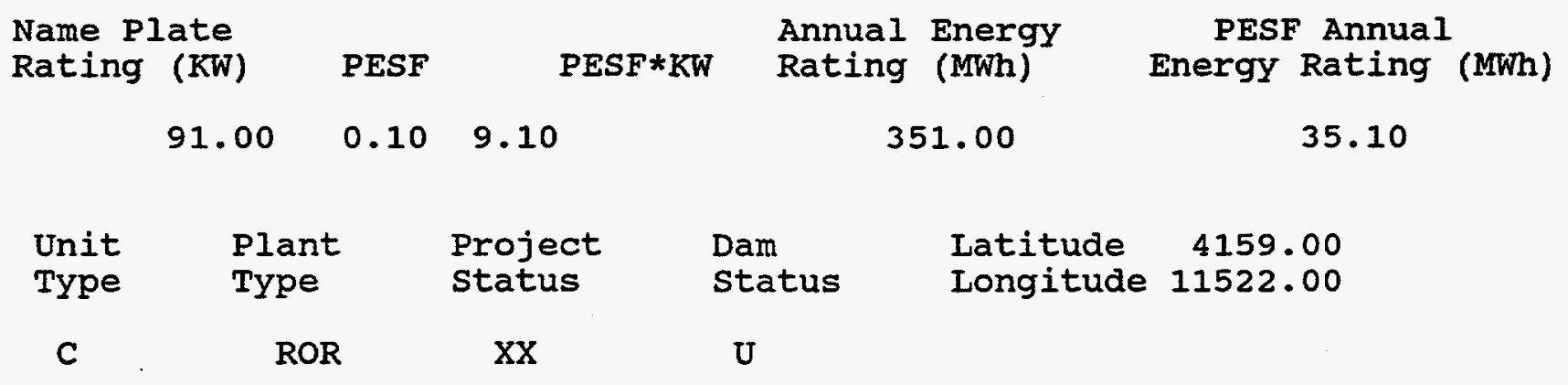

Factor

Exist Prob

Wild/Scenic Protection

wild/Scenic Tributary or Upstream/Downstream wild/Scenic Location Cultural value Fish Presence Value Geologic Value Historic Value other Value

Recreation Value Scenic Value
River Basin

SNAKE RIVER BASIN
State

Name NV
DAVE CREEK

stream 
FERC

Number

Plant Name

NVH0075 JARBIDGE \#1

County Name

ELKO

class Owner Name

C UNKNOWN state

Name

EAST FORK JARBIDGE RIVER

NV
River Basin

SNAKE RIVER BASIN

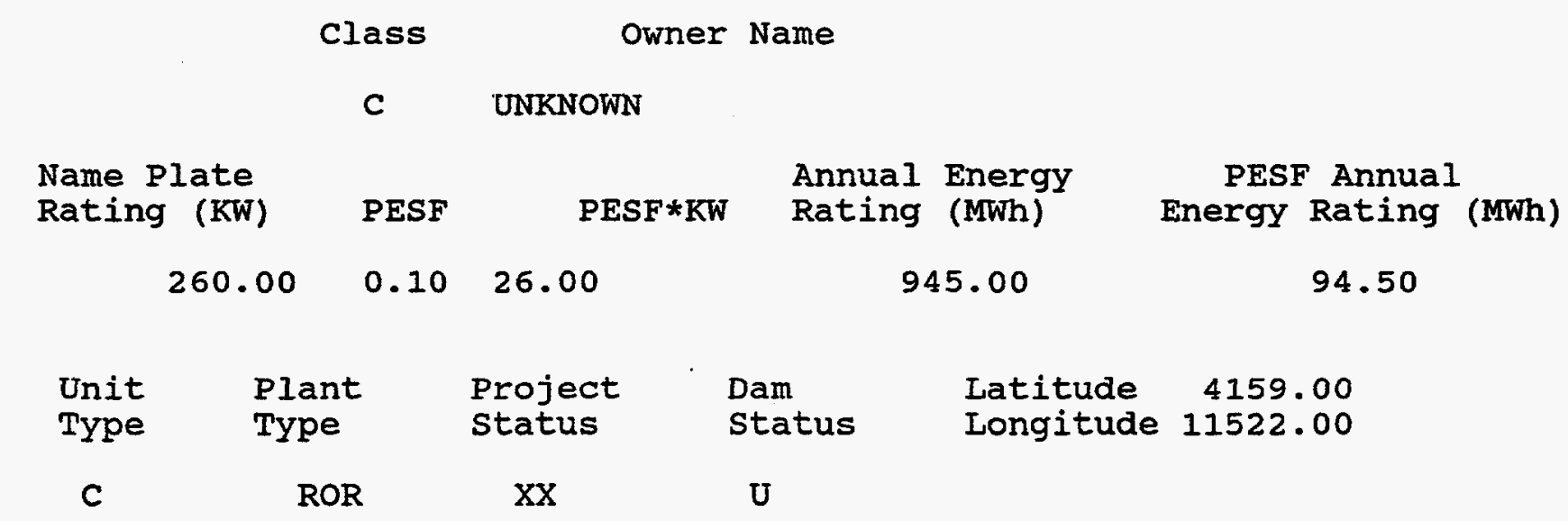

Factor

Wild/Scenic Protection

Wild/Scenic Tributary or Upstream/Downstream wild/Scenic Location Cultural Value Fish Presence Value Geologic Value Historic Value other Value Recreation Value Scenic Value
Exist Prob

$\begin{array}{cc} & 0.90 \\ & \\ & \\ & 0.90 \\ & 0.90 \\ \mathrm{Y} & 0.25 \\ & 0.90 \\ & 0.90 \\ & 0.90 \\ \mathrm{Y} \quad 0.25 \\ & 0.90\end{array}$

Factor

Exist Prob

Wildlife value Threatened/Endangered Fish Threatened/Endangered Wildlife Federal Land Code 103 Federal Land Code 104 Federal Land Code 105 Federal Land Code 106 Federal Land Code 107 Federal Land Code 108 Federal Land Code 198
0.90

0.90

0.90

0.90

$Y$

0.50

0.90

0.90

0.90

0.90

0.90 
DATE: $08 / 19 / 97$

FERC

Number

NVH0076 CANYON PLANT

County Name

ELKO
PAGE NO: 129

State

Name

NV

\section{CANYON CREEK}

River Basin

SNAKE RIVER BASIN

\section{Class Owner Name}

C UNKNOWN

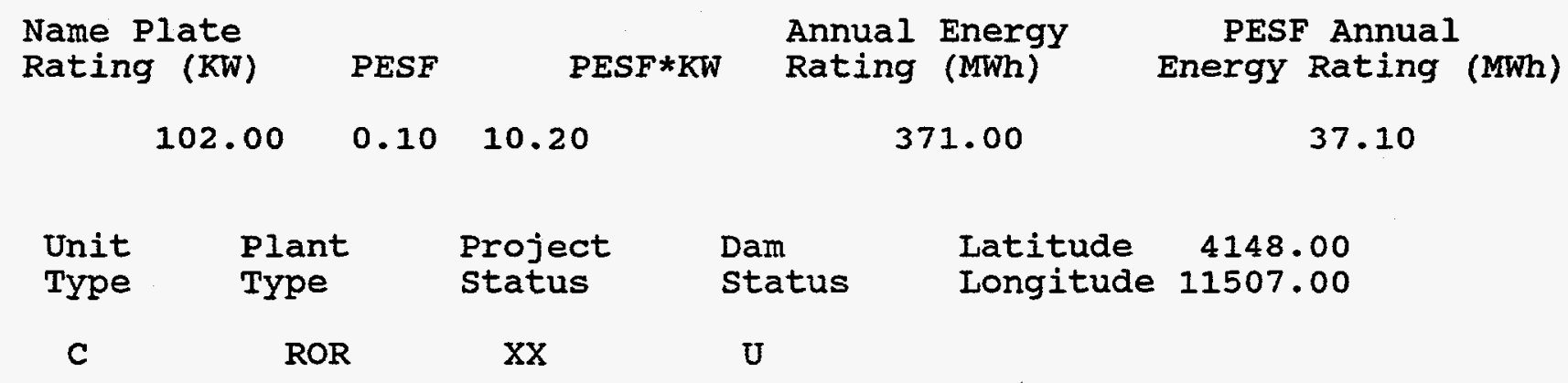

Factor

Wild/Scenic Protection

wild/Scenic Tributary or

Upstream/Downstream

wild/Scenic Location

Cultural Value

Fish Presence Value

Geologic Value

Historic Value

other value

Recreation value

Scenic Value
Exist Prob

Factor

Exist Prob

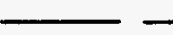

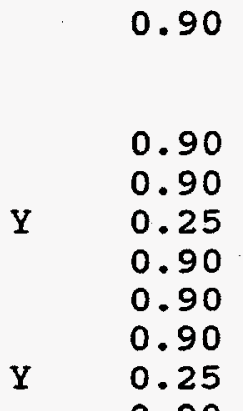

Wildife Value

Threatened/Endangered Fish

Threatened/Endangered Wildlife

Exist Prob

Federal Land Code 103

Federal Land Code 104

Federal Land Code 105

Federal Land Code 106

Federal Land Code 107

Federal Land Code 108

Federal Land Code 198
0.90

0.90

0.90

0.90

$\mathrm{Y}$

0.50

0.90

0.90

0.90

0.90

0.90 

R E S O U R C E
D A T A B A S E
L I S T I N G

DATE: $08 / 19 / 97$

PAGE NO: 130

FERC

Number

Plant Name

stream

State

Name

NVH0080 DIXIE FLATS PLANT

DIXIE CREEK

NV

County Name

River Basin

ELKO

HUMBOLDT RIVER BASIN

Class Owner Name

C IJ.S.D.I. BUREAU OF LAND MANAGEMENT

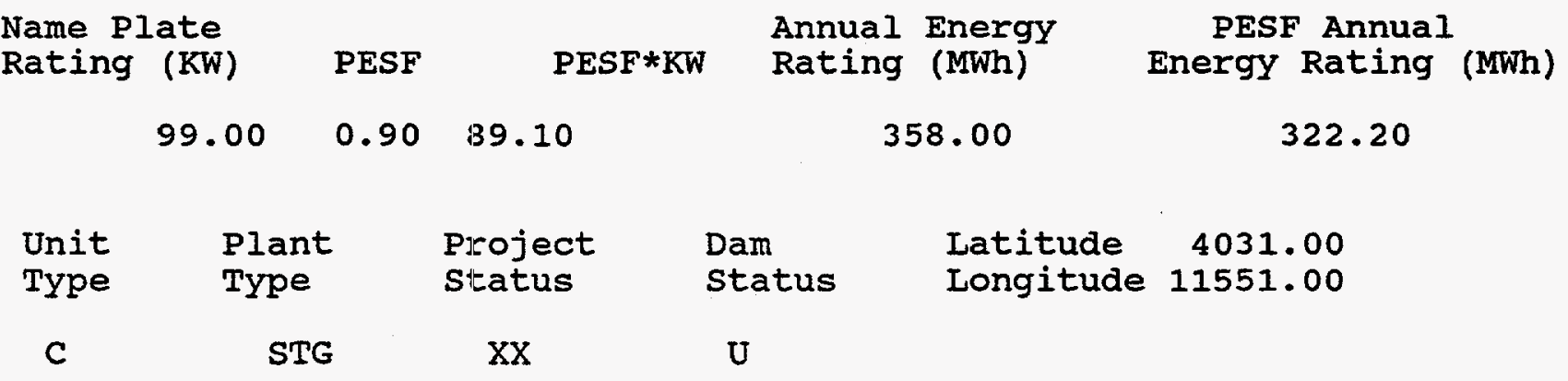

Factor

Exist Prob

Factor

Exist Prob

Wild/Scenic Protection

wild/Scenic Tributary or

0.90 Wildlife Value

Threatened/Endangered Fish

Upstream/Downstream

wild/Scenic Location

0.90 Federal Land Code 103

0.90

0.90

0.90

Federal Land Code 104

0.90

Cultural value

Fish Presence Value

0.90

Federal Land Code 105

0.90

0.90

$\begin{array}{lll}0.90 & \text { Federal Land Code } 106 \\ 0.90 & \text { Federal Land Code } 107\end{array}$

0.90

Geologic Value

0.90

Historic Value

0.90

Federal Land Code 108

0.90

Other value

Recreation value

0.90

Federal Land Code 198

0.90

0.90

0.90

0.90

Scenic Value 
DATE: $08 / 19 / 97$

FERC

Number

Plant Name

NVHO081 FRANKLIN \#3

County Name

ELKO

Class

C

UNKNOWN

Name Plate

Rating ( $\mathrm{KW}$ )

$$
43.00
$$

PESF

Annual Energy

PESF*KW Rating (MWh)

180.00

$0.50 \quad 21.50$

Plant

Type

STG

Project status

$\mathrm{XX}$

Exist Prob

Factor

Exist Prob

Factor

wild/Scenic Protection

wild/Scenic Tributary or Upstream/Downstream wild/Scenic Location Cultural value Fish Presence Value Geologic Value Historic Value other Value

Recreation Value

Scenic Value
PAGE NO: 131

State

Name

NV
River Basin

FRANKLIN LAKE BASIN
PESF Annual Energy Rating (MWh)

90 $\begin{array}{llr}\text { Dam } & \text { Latitude } & 4029.00 \\ \text { Status } & \text { Longitude } & 11522.00\end{array}$

U
0.90 Wildlife Value

Threatened/Endangered Fish Threatened/Endangered Wildlife

0.90 Federal Land Code 103

0.90 Federal Iand Code 104

0.90 Federal Land Code 105

0.90 Federal Land Code 106

0.90 Federal Land Code 107

0.90 Federal Land Code 108

0.90 Federal Land Code 198 
FERC

Number

Plant Name

NVHO083 QUEEN RANCH PLANT

county Name

ESMERALDA state Name

MORRIS CREEK

NV
River Basin

OWENS RIVER BASIN $\begin{array}{cc}\text { Class } & \text { Owner Name } \\ \text { C } & \text { QUEEN VALLEY RANCH }\end{array}$

Name Plate Rating (KW) PESF

Annual Energy

142.00

0.5071 .00
516.00

\section{Rating (MWh)}

PESF Annua1 Energy Rating (MWh) 258

$\begin{array}{cccll}\text { Unit } & \text { Plant } & \text { Project } & \text { Dam } & \text { Latitude } 3753.00 \\ \text { Type } & \text { Type } & \text { Status } & \text { Status } & \text { Longitude 11823.50 } \\ \text { C } & \text { ROR } & \text { XX } & \text { wo } & \end{array}$

Factor

Wild/Scenic Protection wild/Scenic Tributary or Upstream/Downstream wild/scenic Location Cultural value Fish Presence Value Geologic Value Historic Value other Value Recreation Value Scenic Value
Exist Prob

0.90 Wildlife Value

0.90 Federal Land Code 103

0.90 Federal Land Code 104

0.90 Federal Land Code 105

0.90 Federal Iand Code 106

0.90 Federal Land Code 107

$Y \quad 0.75$

0.90

0.90
Exist Prob

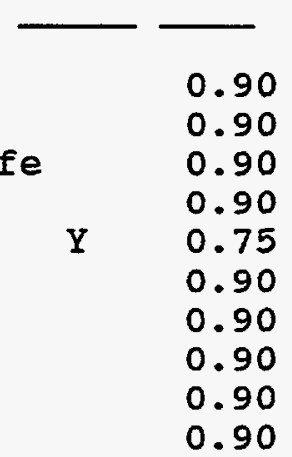


FERC

Number

Plant Name

NVH0085 TRIDENT \#2

County Name

HUMBOLDT

Class

Owner Name

C UNKNOWN
Factor

Name Plate

Rating (KW)

75.00

PESF

PESF*KW

Annual Energy Rating (MWh)

287.00

Latitude

Longitude

Exist Prob

Factor
State

Name

NV
PESF Annual Energy Rating (MWh)

$$
258.30
$$

\begin{tabular}{|c|c|c|c|c|}
\hline $\begin{array}{l}\text { Unit } \\
\text { Type }\end{array}$ & $\begin{array}{l}\text { Plant } \\
\text { Type }\end{array}$ & $\begin{array}{l}\text { Project } \\
\text { Status }\end{array}$ & $\begin{array}{l}\text { Dam } \\
\text { status }\end{array}$ & $\begin{array}{lr}\text { Latitude } & 4156.00 \\
\text { Longitude } & 11829.00\end{array}$ \\
\hline C & ROR & $x x$ & $\mathbf{U}$ & \\
\hline
\end{tabular}

Wild/Scenic Protection

Wild/Scenic Tributary or Upstream/Downstream wild/Scenic Location Cultural value Fish Presence Value Geologic Value Historic Value other Value Recreation Value Scenic Value
Exist Prob

0.90 Wildife Value Threatened/Endangered Fish Threatened/Endangered wildlife

0.90

0.90 Federal Land Code 103 Federal Land Code 104

0.90 Federal Land Code 105

0.90 Federal Land Code 106

0.90 Federal Land Code 107

0.90 Federal Land Code 108

0.90 Federal Land Code 198

0.90 

R E S O U R C E
D A T A B A S E
L I S T I N G

DATE: $08 / 19 / 97$

PAGE NO: 134

FERC

Number

Plant Name

Stream

State

NVHO089 UPPER SOLDIER MEADOW'S

county Name

COLEMAN CREEK

Name

NV

River Basin

HUMBOLDT

BLACK ROCK DESERT BASIN

class Owner Name

F U.S.D.I.BUREAU OF LAND MANAGEMENT

Name Plate

Rating ( $\mathrm{KW}$ )

PESF

Annual Energy

Rating (MWh)

PESF Annual

69.00

$0.90 \quad 62.10$

285.00

256.50

\begin{tabular}{|c|c|c|c|c|}
\hline $\begin{array}{l}\text { Unit } \\
\text { Type }\end{array}$ & $\begin{array}{l}\text { Plant } \\
\text { Type }\end{array}$ & $\begin{array}{l}\text { Project } \\
\text { status }\end{array}$ & $\begin{array}{l}\text { Dam } \\
\text { status }\end{array}$ & $\begin{array}{lr}\text { Latitude } & 4127.00 \\
\text { Longitude } & 11905.00\end{array}$ \\
\hline C & STG & $x x$ & $\mathbf{U}$ & \\
\hline
\end{tabular}

Factor

Exist Prob

Factor

Exist Prob

wild/Scenic Protection

Wild/Scenic Tributary or

0.90 Wildlife Value

Threatened/Endangered Fish

Upstream/Downstream Threatened/Endangered Wildife

0.90

wild/Scenic Location

0.90 Federal Land Code 103

0.90 Federal Land Code 104

0.90 Federal Land Code 105

0.90 Federal Land Code 106

0.90 Federal Land Code 107

0.90 Federal Land Code 108

0.90 Federal Land Code 198

0.90

0.90

Cultural Value

Fish Presence Value

Geologic Value

Historic Value

other value

Recreation Value

0.90

0.90

0.90

0.90

0.90

0.90

0.90

Scenic Value

0.90 
FERC

Number Plant Name

NVH0092 SUMMIT PIANT

county Name

HUMBOLDT

Class Owner Name

C UNKNOWN

Name Plate Rating (KW)

PESF

Annual Energy

PESF*KW Rating (MWh)

258.00
SUMMER CAMP CREEK

River Basin

BLACK ROCK DESERT BASIN

$$
\begin{array}{lll}
67.00 & 0.90 \quad 60.30
\end{array}
$$

state

Name

NV
Project status

XX
Dam status

U
PESF Annual Energy Rating (MWh)

232.20

$\begin{array}{cccc}\text { Unit } & \text { Plant } & \text { Project } & \text { Dam } \\ \text { Type } & \text { Type } & \text { Status } & \text { Status } \\ \text { C } & \text { ROR } & \text { XX } & \text { U }\end{array}$

Factor

Exist Prob
Factor

Latitude
Wild/scenic Protection

Wild/Scenic Tributary or Upstream/Downstream wild/Scenic Location Cultural Value Fish Presence Value Geologic Value Historic Value other Value Recreation Value Scenic Value

\subsection{Wildlife Value} Threatened/Endangered Fish Threatened/Endangered Wildiife Exist Prob

0.90 Federal Land Code 103

0.90 Federal Land Code 104

0.90 Federal Land code 105

0.90 Federal Land Code 106

0.90 Federal Land Code 107

0.90 Federal Land code 108

0.90 Federal Land code 198

0.90
0.90

0.90

0.90

0.90

0.90

0.90

0.90

0.90

0.90

0.90 
FERC

Number

Plant Name

NVH0093 BATTLE CREEK PLANT

County Name

HUMBOLDT
Stream

BATTLE CREEK

River Basin

BLACK ROCK DESERT BASIN

\section{Class Owner Name}

C UNKNOWN

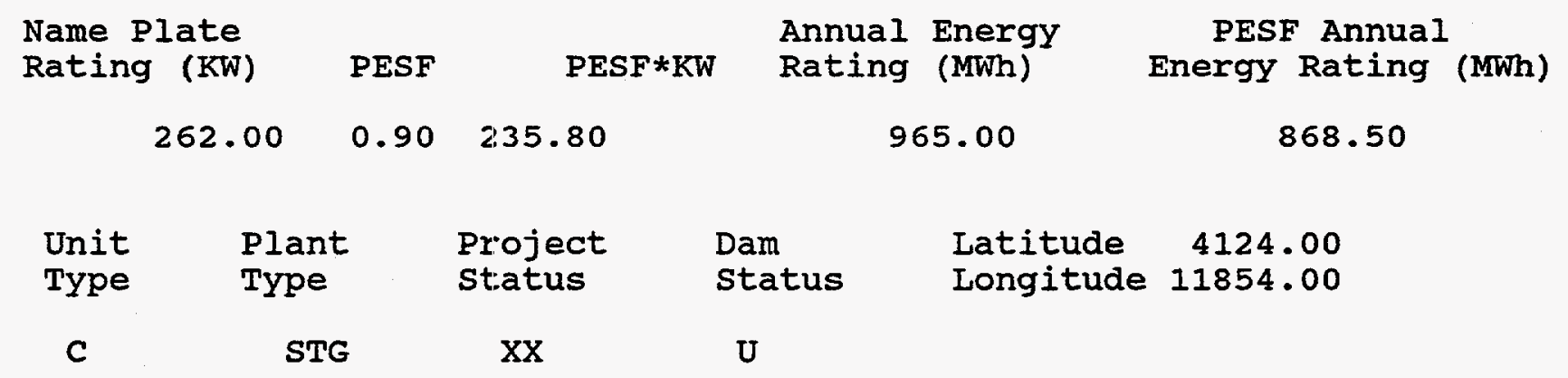

Factor

Exist Prob

0.90

Wildlife value Threatened/Endangered Fish

wild/Scenic Tributary or Upstream/Downstream wild/Scenic Location Cultural value

Fish Presence Value

Geologic Value

Historic Value

other Value

Recreation value

Scenic Value
Factor

Exist Prob
State Name

NV 

R E S O U R C E
D A T A B A S E
I I S T I N G

DATE: $08 / 19 / 97$

PAGE NO: 137

FERC

$\begin{array}{ll}\text { Number } & \text { Plant Name } \\ \text { NVH0095 WILLOW CREEK PLANT }\end{array}$

county Name

HUMBOLDT

\section{stream}

BIG CREEK state

Name

NV

River Basin

BLACK ROCK DESERT BASIN

Class Owner Name

C U.S.D.I. BUREAU OF LAND MANAGEMENT

Name Plate

Rating (KW)

Annual Energy

PESF PESF*KW Rating (MWh)

PESF Annual

130.00

$0.90 \quad 117.00$

499.00

Energy Rating (MWh)

$\begin{array}{cc} & 130.00 \\ \text { Unit } & \text { Plant } \\ \text { Type } & \text { Type } \\ \text { C } & \text { RoR }\end{array}$

Project

status

$\mathrm{XX}$

Dam
status

Latitude

Longitude 11836.00

449.10

Factor

Exist Prob

0.90

Wild/Scenic Protection

Wild/Scenic Tributary or

Upstream/Downstream

wild/Scenic Location

Cultural value

Fish Presence Value

Geologic Value

Historic Value

other Value

Recreation value

Scenic Value

0.90

$\mathrm{U}$

Factor

Exist Prob

Wildlife Value

Threatened/Endangered Fish

Threatened/Endangered Wildlife

0.90 Federal Land Code 103

0.90 Federal Land Code 104

0.90 Federal Land Code 105

0.90 Federal Land Code 106

0.90 Federal Land Code 107

0.90 Federal Land Code 108

0.90 Federal Land Code 198
0.90

0.90

0.90

0.90

0.90

0.90

0.90

0.90

0.90

0.90 
R E S O U R E D A T A B A S E I I S T I N G

DATE: $08 / 19 / 97$

PAGE NO: 138

FERC

Number

Plant Name

Stream

State

Name

NVH0096 REBEL YELL PLANT

REBEL CREEK

NV

county Name

HUMBOLDT

Class

C UNKNOWN

Name Plate

Rating ( $\mathrm{KW}$ )

PESF

PESF*KW

Annual Energy

Rating (MWh)

258.00

67.00

$0.50 \quad 33.50$

Project

Unit

Type

$$
\begin{aligned}
& \text { Plant } \\
& \text { Type }
\end{aligned}
$$

C

ROR

Factor

Exist Prob

0.90

Wild/Scenic Protection

wild/Scenic Tributary or

Upstream/ Downstream

wild/Scenic Location

Cultural value

Fish Presence Value

Geologic Value

Historic Value

other Value

Recreation value

Scenic Value
River Basin

BLACK ROCK DESERT BASIN
Owner Name 

R E S O U R E
D A T A B A S E
L I S T I N G

DATE: $08 / 19 / 97$

PAGE NO: 139

FERC

Number

Plant Name

stream

State

Name

NVHO097 COLONY CREEK PLANT

SINGAS CREEK

NV

county Name

River Basin

HUMBOLDT

HUMBOLDT RIVER BASIN

Class Owner Name

C UNKNOWN

Name Plate

Rating ( $\mathrm{KW})$

PESF

Annual Energy

Rating (MWh)

PESF Annual

52.00

$0.90 \quad 46.80$

214.00

192.60

$\begin{array}{cccll}\text { Unit } & \text { Plant } & \text { Project } & \text { Dam } & \text { Latitude } \\ \text { Type } & \text { Type } & \text { Status } & \text { Status } & \text { Longitude } 11735.00 \\ \text { C } & \text { ROR } & \mathrm{XX} & \mathrm{U} & \end{array}$

Factor

Exist Prob

Factor

Exist Prob

Wild/Scenic Protection

Wild/Scenic Tributary or

0.90 Wildife Value

Threatened/Endangered Fish

Upstream/ Downstream wild/Scenic Location Threatened/Endangered Wildlife

0.90 Federal Land Code 103

0.90 Federal Land Code 104 Energy Rating (MWh)

Cultural Value

Fish Presence Value

0.90 Federal Land Code 105

0.90 Federal Land Code 106

0.90 Federal Land Code 107

0.90 Federal Land Code 108

0.90 Federal Land Code 198

Exist Prob

Historic value

0.90

0.90

0.90

0.90

0.90

0.90

0.90

0.90

0.90

0.90

Recreation Value

Scenic Value

0.90 
RE SOURCE DATABASE L I S T I N G

DATE : $08 / 19 / 97$

PAGE NO: 140

FERC

Number

Plant Name

stream

State

NVH0098 PARADISE \#2

WASH O'NEIL CREEK

Name

NV

County Name

River Basin

HUMBOLDT

HUMBOLDT RIVER BASIN

Class Owner Name

C UNKNOWN

Name Plate

Rating (KW)

PESF

Annual Energy

PESF Annual

53.00

0.90

4.7 .70

207.00

186.30

$\begin{array}{cccll}\text { Unit } & \text { Plant } & \begin{array}{l}\text { Project } \\ \text { Status }\end{array} & \begin{array}{l}\text { Dam } \\ \text { Status }\end{array} & \begin{array}{l}\text { Latitude } \\ \text { Longitude } 11735.00\end{array} \\ \text { Type } & \text { ROR } & \text { XX } & \text { U } & \end{array}$

Factor

Wild/scenic Protection

Wild/Scenic Tributary or Upstream/Downstream wild/scenic Location Cultural value

Fish Presence Value

Geologic Value

Historic Value

other value

Recreation Value

Scenic Value
Exist Prob

0.90

Wildlife Value

Threatened/Endangered Fish

Threatened/Endangered Wildlife

0.90 Federal Land Code 103

0.90 Federal Land Code 104

0.90 Federal Land Code 105

0.90 Federal Land Code 106

0.90 Federal Land Code 107

0.90 Federal Land Code 108

0.90 Federal Land Code 198

0.90

Exist Prob

0.90

0.90

0.90

0.90

0.90

0.90

0.90

0.90

0.90

0.90 
DATE: $08 / 19 / 97$

FERC

Number

Plant Name

NVHO099 STONE HOUSE PLANT

county Name

HUMBOLDT

Class

Owner Name

C UNKNOWN

Name Plate

Rating (KW)

PESF

Annual Energy

PESF*KW

Rating (MWh)

$49.00 \quad 0.90 \quad 44.10$

194.00

Latitude $\quad 4125.00$

Unit

Plant

Project

status

Dam

status

Longitude 11736.00
C ROR

$\mathrm{xx}$

$\mathrm{U}$

Factor

Exist Prob

Factor

Exist Prob

wild/Scenic Protection

wild/Scenic Tributary or Upstream/Downstream wild/Scenic Location

Cultural value

Fish Presence Value

Geologic Value

Historic Value

other Value

Recreation Value

Scenic Value

PAGE NO: 141

State

Name

NV
PESF Annual Energy Rating (MWh)

174.60 
DATE: $08 / 19 / 97$

PAGE NO: 142

FERC

Number

Plant Name

NVH0100 COLEMAN CREEK PLANT

county Name

HUMBOLDT

Stream

State

Name

MULLINEX CREEK

NV

River Basin

HUMBOLDT RIVER BASIN

Class Owner Name

C UNKNOWN

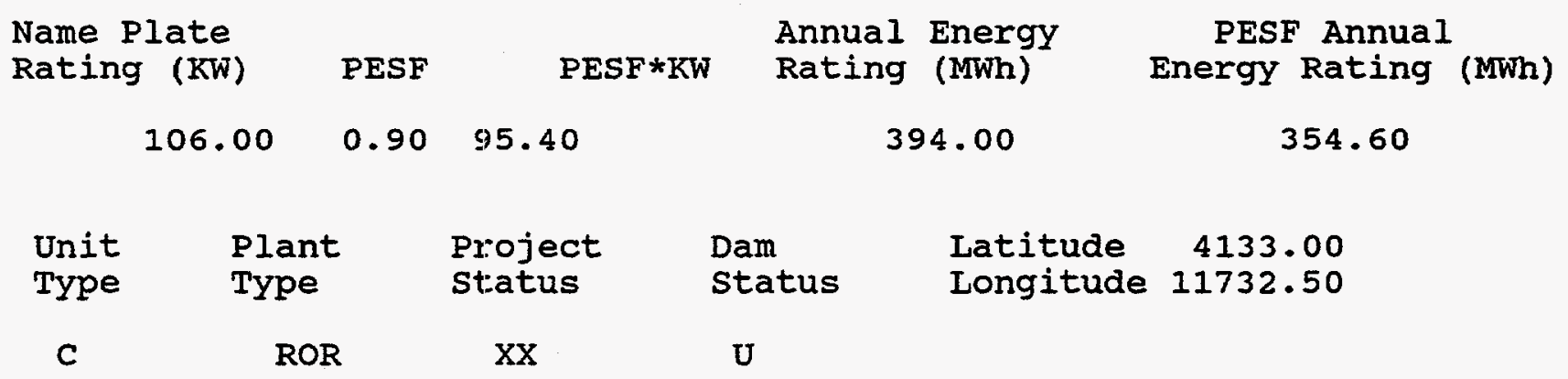

Factor

Wild/Scenic Protection

Wild/Scenic Tributary or Upstream/Downstream wild/Scenic Location Cultural value

Fish Presence Value

Geologic Value

Historic Value

other Value

Recreation value

Scenic Value
Exist Prob

0.90

Wildlife Value

Threatened/Endangered Fish

Threatened/Endangered Wildlife

0.90 Federal Land Code 103

0.90 Federal Land Code 104

0.90 Federal Land Code 105

0.90 Federal Land Code 106

0.90 Federal Land Code 107

0.90 Federal Land Code 108

0.90 Federal Land Code 198
Exist Prob

0.90

0.90

0.90

0.90

0.90

0.90

0.90

0.90

0.90

0.90 
DATE: $08 / 19 / 97$

FERC

Number

Plant Name

NVH0101 AUSTIN-50 PLANT

County Name

LANDER
PAGE NO: 143

stream

BIG CREEK

River Basin

HUMBOLDT RIVER BASIN state

Name

NV

\section{Class Owner Name}

C UNKNOWN

\begin{tabular}{|c|c|c|c|c|c|c|}
\hline \multicolumn{2}{|c|}{ Name Plate } & PESF & $P E S F * K W$ & $\begin{array}{l}\text { Annua } \\
\text { Ratir }\end{array}$ & $\begin{array}{l}\text { Energy } \\
(M W h)\end{array}$ & $\begin{array}{l}\text { PESF Annual } \\
\text { Energy Rating (MWh) }\end{array}$ \\
\hline & 63.00 & 0.50 & 31.50 & & 245.00 & 122.50 \\
\hline $\begin{array}{l}\text { Unit } \\
\text { Type }\end{array}$ & $\begin{array}{l}\text { Pla } \\
\text { TYI }\end{array}$ & & $\begin{array}{l}\text { Project } \\
\text { status }\end{array}$ & $\begin{array}{l}\text { Dam } \\
\text { status }\end{array}$ & $\begin{array}{l}\text { Latitude } \\
\text { Longitude }\end{array}$ & $\begin{array}{r}3921.00 \\
11709.00\end{array}$ \\
\hline C & & & $X X$ & $\mathrm{U}$ & & \\
\hline
\end{tabular}

Factor

Exist Prob

Factor

Exist Prob

Wild/Scenic Protection

Wild/Scenic Tributary or

Upstream/Downstream

wild/Scenic Location

Cultural Value

Fish Presence Value

Geologic Value

Historic Value

other value

Recreation value

0.90 Wildife Value

Threatened/Endangered Fish

Threatened/Endangered wildlife

0.90 Federal Land Code 103

0.90 Federal Land Code 104

0.90 Federal Land Code 105

0.90 Federal Iand Code 106

0.90 Federal Land Code 107

0.90 Federal Land Code 108

0.90 Federal Land Code 198

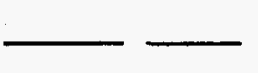

0.90

0.90

0.90

0.90

0.90

$\mathrm{Y} \quad 0.50$

0.90

0.90

0.90

0.90

0.90

Scenic Value 
FERC

Number

Plant Name

NVH0102 SPENCER PLANT

County Name

IANDER

\section{stream}

BIRCH CREEK

River Basin

BIG SMOKY BASIN state Name

NV

\section{Class Owner Name}

C UNKNOWN

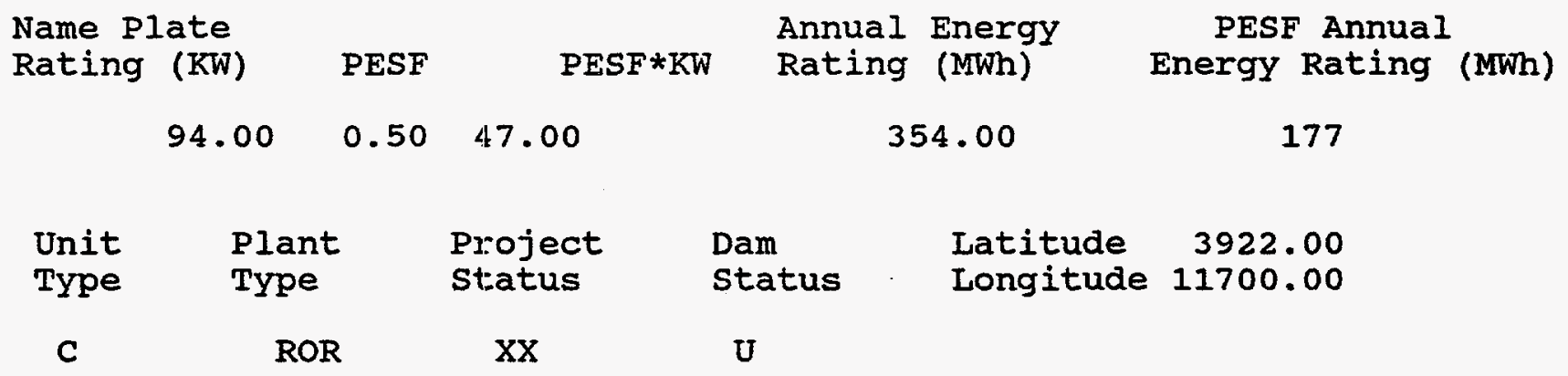

Factor

Wild/Scenic Protection Wild/Scenic Tributary or Upstream/ Downstream wild/Scenic Location Cultural value Fish Presence Value Geologic Value Historic Value other Value Recreation Value Scenic Value
Exist Prob

0.90

Wildlife Value Threatened/Endangered Fish Threatened/Endangered Wildlife

0.90 Federal Land Code 103

0.90 Federal Land code 104

0.90 Federal Land Code 105

0.90 Federal Land Code 106

0.90 Federal Land Code 107

0.90 Federal Land Code 108

0.90 Federal Land Code 198
Exist Prob

0.90

0.90

0.90

0.90

$\mathbf{Y}$

0.90

0.90

0.90

0.90

0.90 
DATE: $08 / 19 / 97$

FERC

Number

Plant Name

NVHO103 MILLET PLANT

County Name

LANDER
PAGE NO: 145

State

Name

NV
BOWMAN CREEK

River Basin

BIG SMOKY BASIN

Class Owner Name

F U.S.D.A. FOREST SERVICE

Name Plate

Rating ( $\mathrm{KW}$ )

PESF

Annual Energy

PESF*KW Rating (MWh)

PESF Annual

45.00

$0.50 \quad 22.50$

191.00

Energy Rating (MWh)

95.50

\begin{tabular}{|c|c|c|c|c|}
\hline $\begin{array}{l}\text { Unit } \\
\text { Type }\end{array}$ & $\begin{array}{l}\text { Plant } \\
\text { Type }\end{array}$ & $\begin{array}{l}\text { Project } \\
\text { Status }\end{array}$ & $\begin{array}{l}\text { Dam } \\
\text { status }\end{array}$ & $\begin{array}{lr}\text { Latitude } & 3810.00 \\
\text { Longitude } 11707.00\end{array}$ \\
\hline C & ROR & $x x$ & U & \\
\hline
\end{tabular}

Factor

Exist Prob

0.90

Wild/Scenic Protection

Wild/Scenic Tributary or Upstream/Downstream wild/Scenic Location

Cultural Value

Fish Presence Value

Geologic Value

Historic Value

other Value

Recreation value

Scenic Value
Factor

Exist Prob

Wildife Value

Threatened/Endangered Fish

Threatened/Endangered Wildlife

0.90 Federal Land Code 103

0.90 Federal Land Code 104

0.90 Federal Land Code 105

0.90 Federal Land Code 106

0.90 Federal Land Code 107

0.90 Federal Land Code 108

0.90 Federal Land Code 198 
RESOURCE D A T A B A E I I S T I N G

DATE: $08 / 19 / 97$

PAGE NO: 146

FERC

Number

Plant Name

Stream

State

NVH0105 CALLAGHAN \#2

STEINER CREEK

Name

NV

County Name

River Basin

LANDER

GRASS BASIN

Class Owner Name

R UNKNOWN

Name Plate

Rating (KW) PESF PESF*KW Rating (MWh)

Annual Energy

PESF Annual

110.00

$0.90 \quad 99.00$

432.00

Energy Rating (MWh)

$\begin{array}{lll}110.00 & 0.90 \quad 99.00\end{array}$

$\begin{array}{ll}\text { Unit } & \text { Plant } \\ \text { Type } & \text { Type }\end{array}$

Project

status

Dam

Status

Latitude 3939.00

c

ROR

$\mathrm{xx}$

U

Factor

Wild/Scenic Protection

Wild/Scenic Tributary or

Upstream/Downstream

wild/Scenic Location

Cultural Value

Fish Presence Value

Geologic Value

Historic Value

other Value

Recreation value

Scenic Value
Exist Prob

0.90 Wildlife Value

Threatened/Endangered Fish

Threatened/Endangered Wildlife

0.90 Federal Land code 103

0.90 Federal Land Code 104

0.90 Federal Land Code 105

0.90 Federal Land Code 106

0.90 Federal Land Code 107

0.90 Federal Land Code 108

0.90 Federal Land Code 198
388.80

0.90

Exist Prob

0.90

0.90

0.90

0.90

0.90

0.90

0.90

0.90

0.90

0.90 
FERC

Number

Plant Name

NVH0106 PINE GROVE PLANT

county Name

LYON

Class Owner Name

F U.S.D.A. FOREST SERVICE

Name Plate

Rating ( $K W$ )

793.00

PESF

Annual Energy

PESF*KW

Rating (MWh)

$0.50 \quad 396.50$

3000.00

Unit

Plant

Type

Type

C

STG

Factor

Exist Prob

Factor

Exist Prob

Wild/Scenic Protection

Wild/Scenic Tributary or

Upstream/Downstream

wild/Scenic Location

cultural value

Fish Presence Value

Geologic Value

Historic Value

other Value

Recreation Value

Scenic Value
Latitude $\quad 3835.00$

Project

status

$\mathrm{XX}$

Dam
status

Longitude 11913.00

$\mathrm{U}$
State

Name

NV
PESF Annual
Energy Rating (MWh)
1500

$\begin{array}{lll}0.90 & \text { Wildlife Value } & 0.90 \\ & \text { Threatened/Endangered Fish } & 0.90 \\ 0.90 & \text { Threatened/Endangered Wildlife } & 0.90 \\ 0.90 & \text { Federal Land Code 103 } & 0.90 \\ 0.90 & \text { Federal Land Code } 104 & \\ 0.90 & \text { Federal Land Code } 105 & 0.50 \\ 0.90 & \text { Federal Land Code 106 } & 0.90 \\ 0.90 & \text { Federal Land Code 108 } & 0.90 \\ 0.90 & \text { Federal Land Code } 198 & 0.90 \\ 0.90 & & 0.90 \\ & & 0.90\end{array}$




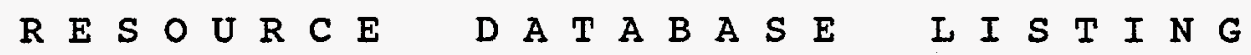

DATE: $08 / 19 / 97$

PAGE NO: 148

FERC

Number

Plant Name

stream

State

Name

NVH0108 BODIE PLANT

BODIE CREEK

NV

county Name

River Basin

MINERAL

WALKER IAKE BASIN

\section{Class Owner Name}

C INNKNOWN

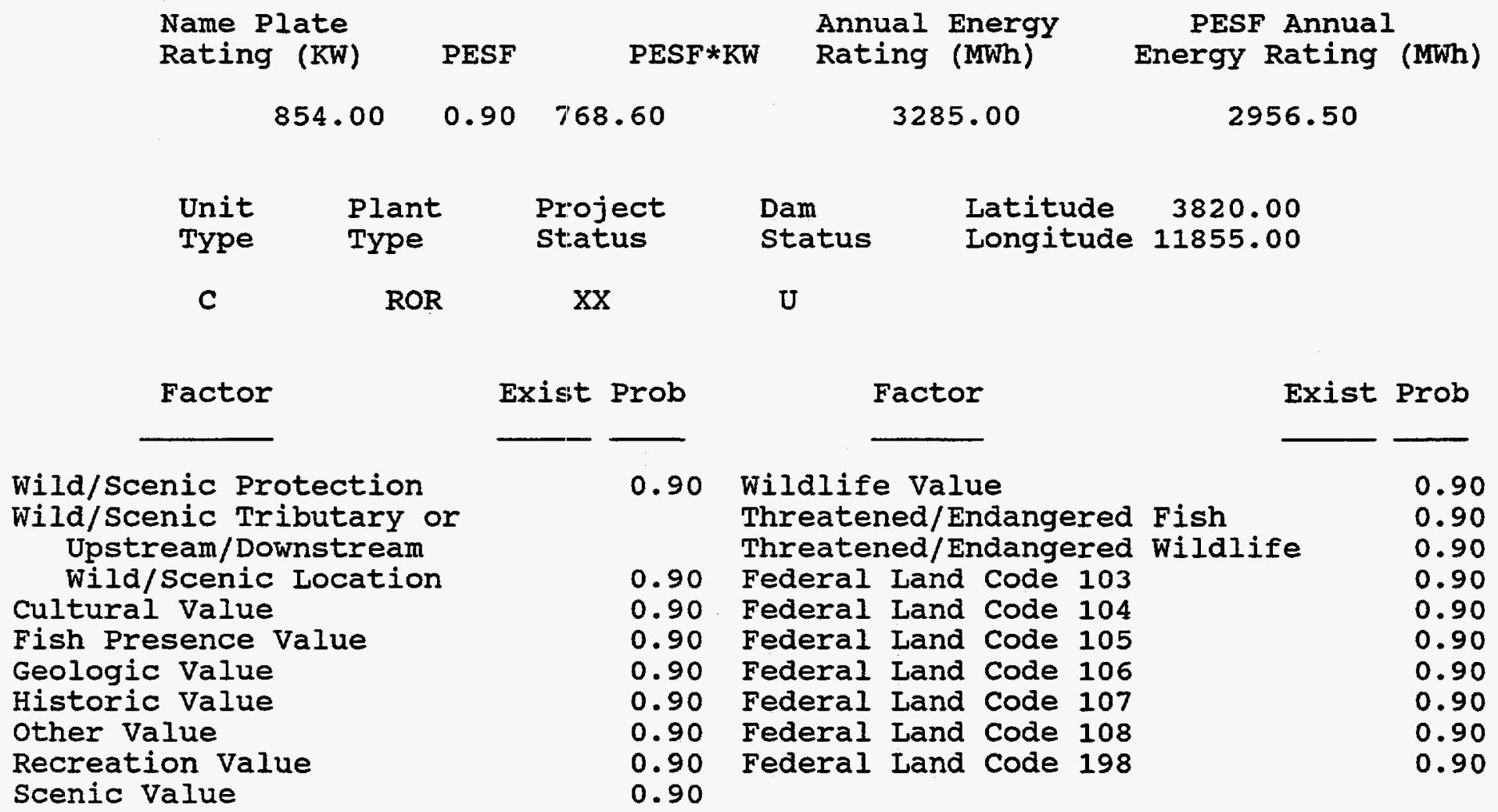


R E S O U R C E

DATE: $08 / 19 / 97$
D A T A B A S E L I S T I N G

PAGE NO: 149

FERC

Number

Plant Name

NVH0109 ROUGH WATER PLANT

county Name

MINERAL

Class

Owner Name

C UNKNOWN
River Basin

WALKER LAKE BASIN
State

Name

NV

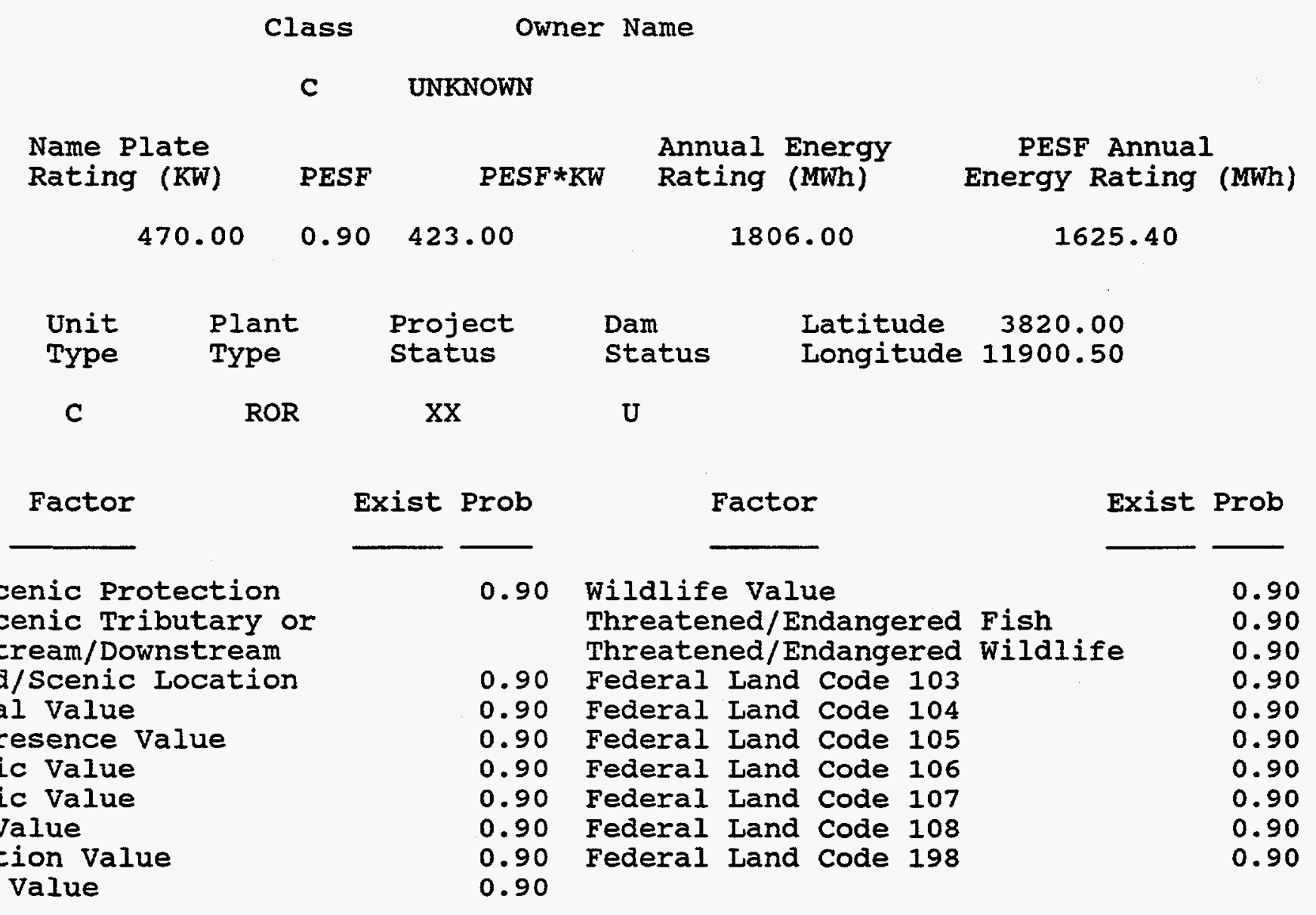

Wild/scenic Protection

Wild/Scenic Tributary or Upstream/Downstream wild/Scenic Location

cultural value

Fish Presence Value

Geologic Value

Historic Value

other Value

Recreation Value

Scenic Value 

R E S O U R C E
D A T A B A S E
L I T ING

DATE: $08 / 19 / 97$

PAGE NO: 150

FERC

Number

Plant Name

stream

State

Name

NVH0110 COTTONWOOD

COTTONWOOD CREEK

NV

county Name

MINERAL
River Basin

WALKER LAKE BASIN
Class Owner Name

F U.S. ARMY
Name Plate Rating (KW)

1372.00
PESF

0.50

686.00

Project status

Unit
Type

$$
\text { Plant }
$$
Type

c STG

XX
Annual Energy Rating (MWh)

5262.00
PESF Annual Energy Rating (MWh)

Dam

status

Latitude $\quad 3838.50$

U

Factor

Wild/Scenic Protection

Wild/Scenic Tributary or

Upstream/Downstream

wild/Scenic Location

Cultural Value

Fish Presence Value

Geologic Value

Historic Value

other value

Recreation value

Scenic Value
Exist Prob

0.90

Wildlife Value

Threatened/Endangered Fish Threatened/Endangered Wildlife

0.90 Federal Land Code 103

0.90 Federal Land Code 104

0.90 Federal Land Code 105

0.90 Federal Land Code 106

0.90 Federal Land Code 107

0.90 Federal Land Code 108

0.90 Federal Land Code 198
Exist Prob

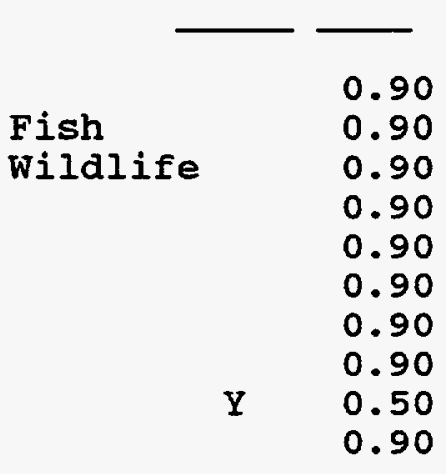



R E S O U R C E
D A T A B A $S$ E
I I S T I N G

DATE: $08 / 19 / 97$

PAGE NO: 151

FERC

Number

Plant Name

Stream

state

Name

NVH0112 CORRAL WASH PLANT

CLEAR CREEK

NV

County Name

River Basin

NYE

HUMBOLDT RIVER BASIN

Class Owner Name

F U.S.D.A. FOREST SERVICE

Name Plate

$\begin{array}{lll}\text { PESF } & \text { PESF*KW } & \begin{array}{l}\text { Annual Energy } \\ \text { Rating }\end{array} \\ \text { (MWh) }\end{array}$

PESF Annual

Rating (KW)

$0.50 \quad 20.50$

162.00

Energy Rating (MWh)

41.00

20.50

81

Unit Plant

Type Type

c

ROR

Exist Prob
Dam
Status

$\begin{array}{lr}\text { Latitude } & 3859.00 \\ \text { Longitude } & 11727.00\end{array}$

status

$\mathrm{XX}$

U
Factor

\begin{abstract}
Factor
Wild/Scenic Protection

Wild/scenic Tributary or Upstream/Downstream wild/Scenic Location Cultural value

Fish Presence Value

Geologic Value

Historic Value

other value

Recreation value

Scenic value
\end{abstract}

(1)

0.90
0.90
0.90
0.90
0.90
0.90
0.90
0.90
0.90

Wildlife Value Threatened/Endangered Fish

Exist Prob Threatened/Endangered Wildlife Federal Land Code 103 Federal Land Code 104 Federal Land Code 105 Federal Land Code 106 Federal Land Code 107 Federal Land code 108 Federal Land Code 198

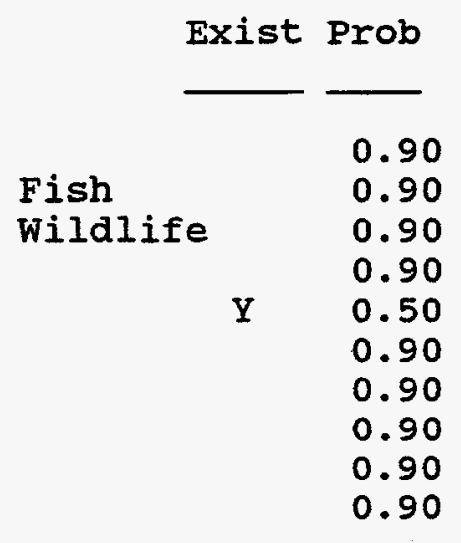



R E S O U R C E
D A T A B A S E
L I S T I N G

DATE: $08 / 19 / 97$

PAGE NO: 152

FERC

Number

Plant Name

NVH0115 PABLO CANYON PLANT

County Name

NYE

\section{Class}

F

U.S.D.A. FOREST SERVICE

Name Plate

Rating ( $\mathrm{KW}$ )

PESF

95.00

$0.50 \quad 47.50$

PESF*KW

Annual Energy

Rating (MWh)

367.00

Latitude $\quad 3841.50$

Unit

Plant

Type

c

ROR

Factor

Exist Prob

Factor

Exist Prob

Wild/Scenic Protection

Wild/Scenic Tributary or Upstream/Downstream wild/Scenic Location Cultural Value

Fish Presence Value

$$
\text { Project }
$$
status

$\mathrm{xX}$

Status

Longitude 11713.00
State

Name

NV
PESF Annual Energy Rating (MWh)

$$
183.50
$$

$\mathrm{U}$
Geologic Value

Historic Value

other value

Recreation Value

Scenic Value
0.90 Wildlife Value

Threatened/Endangered Fish Threatened/Endangered Wildlife

0.90 Federal Land code 103

0.90 Federal Iand Code 104

0.90 Federal Land Code 105

0.90 Federal Land Code 106

0.90 Federal Land Code 107

0.90 Federal Land Code 108

0.90 Federal Land Code 198
0.90

0.90

0.90

0.90

$\mathrm{Y} \quad 0.50$

0.90

0.90

0.90

0.90

0.90 
DATE: $08 / 19 / 97$

FERC

Number

Plant Name

NVH0116 BARKER PLANT

County Name

NYE

Class

F

U.S.D.I

Owner Name

BARKER CREEK

stream

PAGE NO: 153

State

Name

NV

BIG SMOKY BASIN

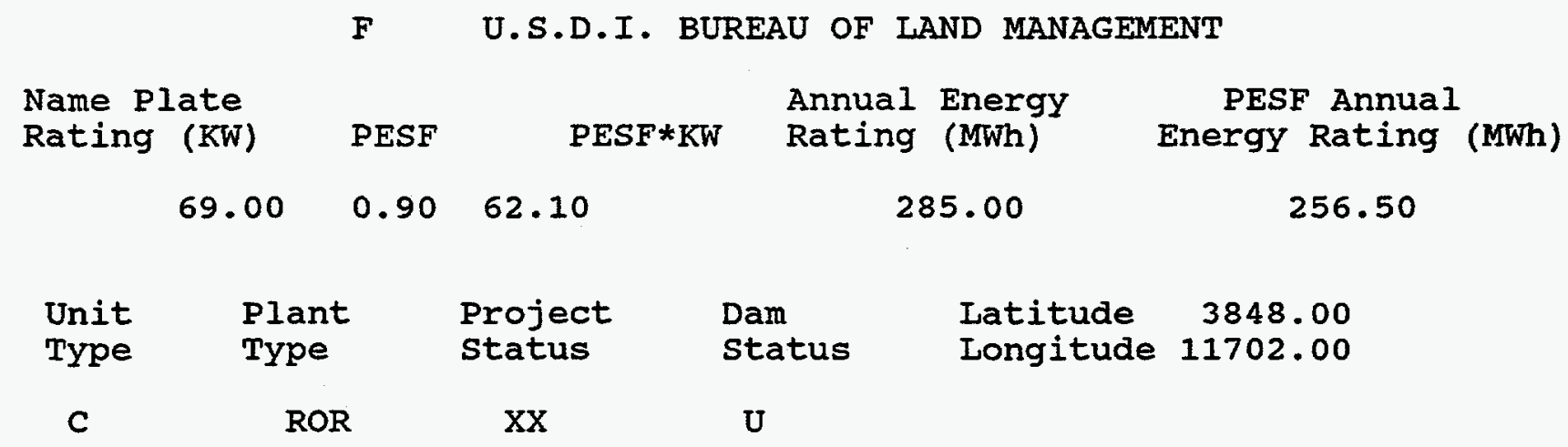

Factor

Exist Prob

Factor

Exist Prob

Wild/Scenic Protection

Wild/scenic Tributary or

Upstream/Downstream

Wild/Scenic Location

Cultural value

Fish Presence Value

Geologic Value

Historic Value

other Value

Recreation Value

Scenic Value

0.90 Wildlife Value
Threatened/Endangered Fish

Threatened/Endangered Wildlife

0.90 Federal Land Code 103

0.90 Federal Land Code 104

0.90 Federal Land Code 105

0.90 Federal Land code 106

0.90 Federal Land Code 107

0.90 Federal Land Code 108

0.90 Federal Land Code 198
0.90

0.90

0.90

0.90

0.90

0.90

0.90

0.90

0.90

0.90 
FERC

Number

Plant Name

NVHO117 NOT P.C. PLANT

county Name

NYE

Class

Owner Name

F

U.S.D.A. FOREST SERVICE

Name Plate

Rating ( $\mathrm{KW}$ )

104.00

PESF

Annual Energy

PESF * KW

Rating (MWh)

$0.50 \quad 52.00$

427.00

Latitude $\quad 3846.00$

Unit Plant

Type Type

c

STG

Project

status

$\mathrm{xX}$

Dam

Status

Longitude 11712.00

$\mathrm{U}$
State

Name

NV
PESF Annual Energy Rating (MWh)

Factor

Exist Prob

Factor

Exist Prob

213.50

Wild/Scenic Protection

wild/Scenic Tributary or Upstream/Downstream wild/Scenic Location Cultural value

Fish Presence Value

Geologic Value

Historic Value other Value

Recreation value

Scenic Value
0.90 Wildife Value

Threatened/Endangered Fish Threatened/Endangered Wildlife

0.90 Federal Land Code 103

0.90 Federal Land Code 104

0.90 Federal Land Code 105

0.90 Federal Land Code 106

0.90 Federal Land Code 107

0.90 Federal Land Code 108

0.90 Federal Land Code 198

0.90

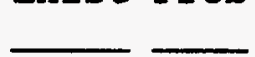

0.90

0.90

0.90

0.90

0.50

0.90

0.90

0.90

0.90

0.90 
DATE: $08 / 19 / 97$

FERC

Number Plant Name

NVHO118 COVE PLANT

County Name

NYE
PAGE NO: 155

State

Name

COVE CANYON CREEK

NV

Class Owner Name

F U.S.D.A. FOREST SERVICE

Name Plate

Rating (KW)

PESF

Annual Energy

$67.00 \quad 0.50 \quad 33.50$

BIG SMOKY BASIN

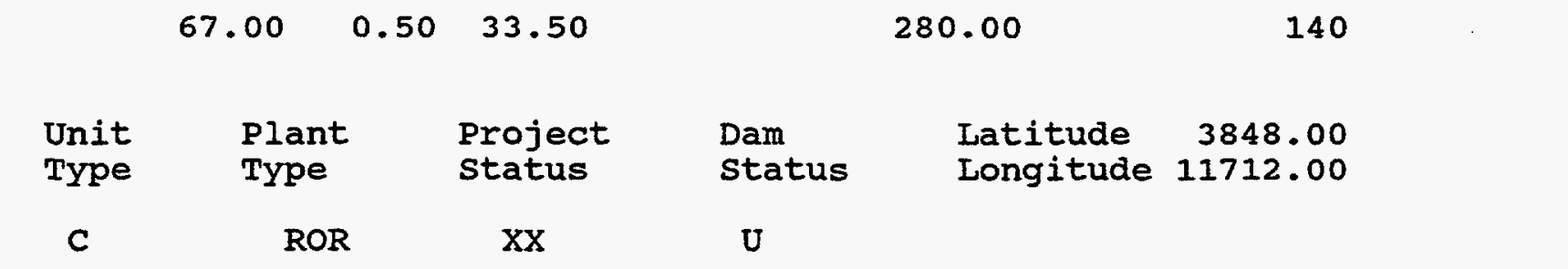

Factor

Exist Prob

Factor

Exist Prob

Wild/Scenic Protection

wild/Scenic Tributary or

Upstream/ Downstream

wild/Scenic Location

Cultural value

Fish Presence Value

Geologic Value

Historic Value

other Value

Recreation Value

Scenic Value

0.90 Wildife Value

Threatened/Endangered Fish

Threatened/Endangered Wildlife

0.90 Federal Land Code 103

0.90 Federal Land Code 104

0.90 Federal Land Code 105

0.90 Federal Land Code 106

0.90 Federal Land Code 107

0.90 Federal Land Code 108

0.90 Federal Land Code 198

0.90
PESF Annual

Energy Rating (MWh)

140 


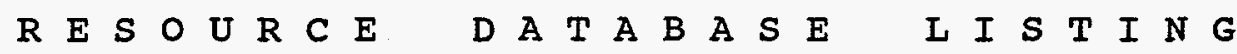

DATE: $08 / 19 / 97$

PAGE NO: 156

FERC

Number

Plant Name

NVH0119 ARC DOME \#2

NORTH TWIN RIVER

State

Name

NV

county Name

NYE

Class

F U.S.D.A. FOREST SERVICE

Name Plate

Rating (KW)

134.00

PESF

Annual Energy

PESF*KW Rating (MWh)

516.00
River Basin

BIG SMOKY BASIN

$\begin{array}{ccccc}\text { Unit } & \text { Plant } & \text { Project } & \text { Dam } & \text { Latitude } \\ \text { Type } & \text { Type } & \text { Status } & \text { Status } & \text { Longitude } 11715.00 \\ \text { C } & \text { STG } & \text { XX } & \text { U } & \end{array}$

Factor

Wild/Scenic Protection

Wild/Scenic Tributary or

Upstream/Downstream

wild/Scenic Location

Cultural Value

Fish Presence Value

Geologic Value

Historic Value

other Value

Recreation Value

Scenic Value
Exist Prob

0.90

Wildlife Value

Threatened/Endangered Fish

Threatened/Endangered Wildlife

0.90 Federal Land Code 103

0.90 Federal Land Code 104

0.90 Federal Land Code 105

0.90 Federal Land Code 106

0.90 Federal Land Code 107

0.90 Federal Land Code 108

0.90 Federal Land Code 198
PESF Annual Energy Rating (MWh)

258

0.90

Exist Prob

0.90

0.90

0.90

0.90

$Y \quad 0.50$

0.90

0.90

0.90

0.90

0.90 
DATE: $08 / 19 / 97$

PAGE NO: 157

State

Name

NV
FERC

Number

NVH0120 LAST CHANCE PLANT

County Name

NYE

Plant Name

\author{
county Name
}

NY $\begin{array}{cc}\text { Class } & \text { Owner Name } \\ \text { F } & \text { U.S.D.A. FOREST SERVICE }\end{array}$

Name Plate

Rating ( $\mathrm{KW}$ )

78.00

PESF

Annual Energy

PESF*KW

Rating (MWh)

River Basin

BIG SMOKY BASIN

\section{LAST CHANCE CREEK}

321.00

$0.50 \quad 39.00$ $\begin{array}{ll}\text { Unit } & \text { Plant } \\ \text { Type } & \text { Type }\end{array}$

c

ROR

Factor

Exist Prob

0.90

Wild/Scenic Protection

wild/scenic Tributary or

Upstream/Downstream

wild/Scenic Location

Cultural Value

Fish Presence Value

Geologic Value

Historic Value

other Value

Recreation Value

Scenic Value

$\begin{array}{lllr}\text { Project } & \text { Dam } & \text { Latitude } & 3856.00 \\ \text { Status } & \text { Status } & \text { Longitude } & 11715.00\end{array}$

$\mathrm{U}$
PESF Annual Energy Rating (MWh)

160.50
Factor

Exist Prob

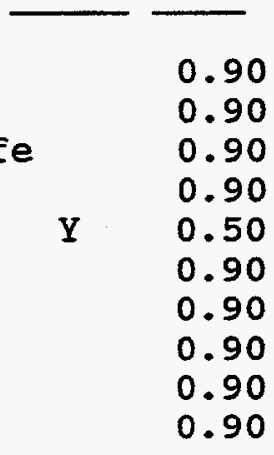



R E S O U R C E
D A T A B A S E
L I S I N G

DATE: $08 / 19 / 97$

PAGE NO: 158

FERC

Number

Plant Name

Stream

State

NVH0121 GOLDRUSH PLANT

OPHIR CREEK

Name

NV

county Name

NYE
Name Plate Rating ( $\mathrm{KW}$ )

78.00
PESF

U.S.D.A. FOREST SERVICE

BIG SMOKY BASIN
River Basin

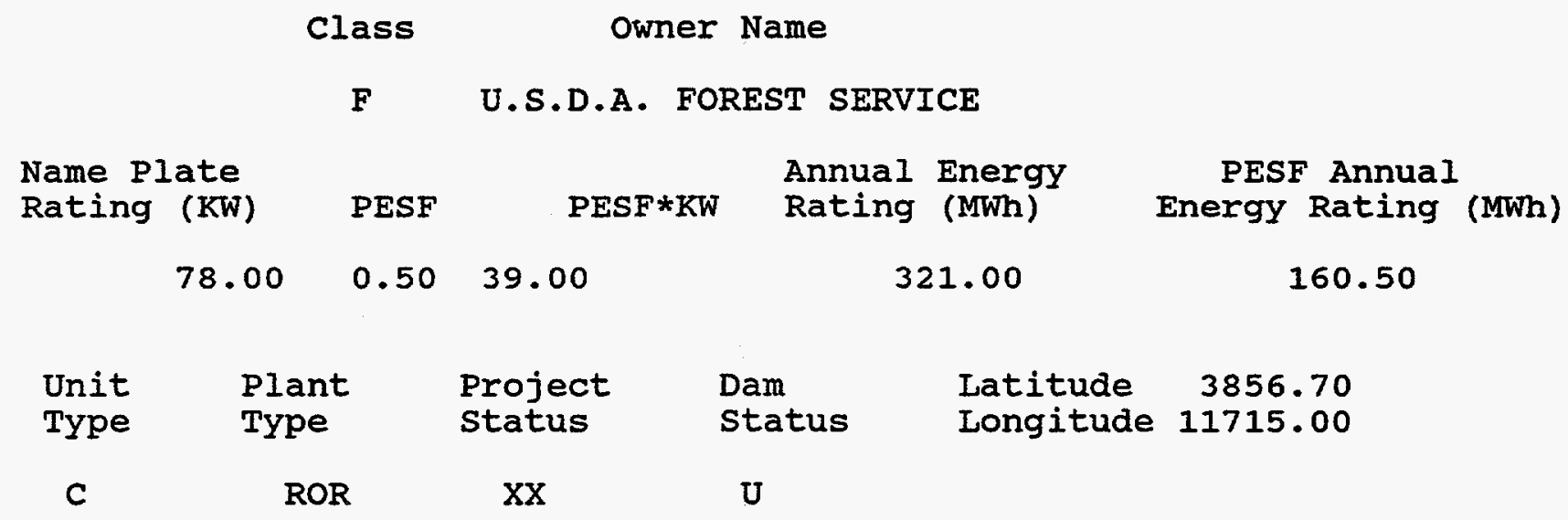

Factor

Wild/Scenic Protection

wild/Scenic Tributary or

Upstream/Downstream

wild/Scenic Location

Cultural Value

Fish Presence Value

Geologic Value

Historic Value

other value

Recreation value

Scenic Value
Exist Prob

0.90

Wildife value

Threatened/Endangered Fish

Threatened/Endangered Wildiife

0.90 Federal Land Code 103

0.90 Federal Land Code 104

0.90 Federal Land Code 105

0.90 Federal Land Code 106

0.90 Federal Land Code 107

0.90 Federal Land Code 108

0.90 Federal Land Code 198
Exist Prob

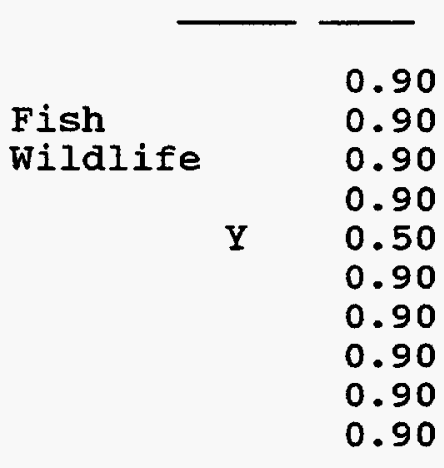



R E S O U R C E
D A T A B A S E
L I S T I N G

DATE: $08 / 19 / 97$

PAGE NO: 159

FERC

Number Plant Name

NVH0122 TEXTILE PIANT

county Name

River Basin

NYE

LITTLE FISH LAKE BASIN

Class Owner Name

F UNKNOWN

Name Plate

Annual Energy

Rating (KW)

PESF

PESF*KW

Rating (MWh)

PESF Annual

96.00

0.5048 .00

378.00

189

Unit Plant Project Dam Latitude 3846.00

Type Type status status Longitude 11631.00

C ROR $\quad \mathrm{XX} \quad \mathrm{U}$

Factor

Exist Prob

Factor

Exist Prob

wild/scenic Protection

wild/scenic Tributary or

Upstream/Downstream

wild/Scenic Location

0.90 Wildlife Value

Threatened/Endangered Fish

state

Name

NV

Energy Rating (MWh)

Threatened/Endangered WildIife

0.90 Federal Land code 103

0.90 Federal Land code 104

0.90 Federal Land code 105

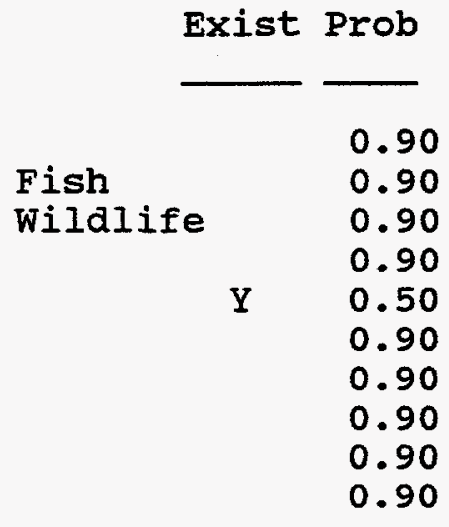

Cultural Value

0.90 Federal Land code 106

0.90 Federal Land Code 107

0.90 Federal Land Code 108

0.90 Federal Land Code 198

0.90

Recreation Value

Scenic Value

0.90 
R E S O UR C E D A T A B A S E I I S T IN

DATE: $08 / 19 / 97$

PAGE NO: 160

FERC

Number

Plant Name

stream

State

NVH0123 WINDOW PLANT

CLEAR CREEK

Name

NV

county Name

NYE

$$
\text { Class Owner Name }
$$

C UNKNOWN

\begin{tabular}{|c|c|c|c|c|c|c|}
\hline \multirow[t]{2}{*}{$\begin{array}{l}\text { Name } P \\
\text { Rating }\end{array}$} & $\begin{array}{l}\text { late } \\
(\mathrm{KW})\end{array}$ & PESF & $\mathrm{PESF} * \mathrm{KW}$ & $\begin{array}{l}\text { Annu } \\
\text { Rati }\end{array}$ & $\begin{array}{l}\text { Energy } \\
\text { (MWh) }\end{array}$ & $\begin{array}{l}\text { PESF Annual } \\
\text { Energy Rating (MWh) }\end{array}$ \\
\hline & 82.00 & 0.50 & 41.00 & & 327.00 & 163.50 \\
\hline $\begin{array}{l}\text { Unit } \\
\text { Type }\end{array}$ & $\begin{array}{l}\text { Pla } \\
\text { TyY }\end{array}$ & & $\begin{array}{l}\text { Project } \\
\text { Status }\end{array}$ & $\begin{array}{l}\text { Dam } \\
\text { status }\end{array}$ & $\begin{array}{l}\text { Latitude } \\
\text { Longitude }\end{array}$ & $\begin{array}{r}3849.00 \\
11631.00\end{array}$ \\
\hline$c$ & & & $x X$ & $\mathrm{U}$ & & \\
\hline
\end{tabular}

Factor

Exist Prob

Factor

Exist Prob

0.90 Wildife Value

Threatened/Endangered Fish

Threatened/Endangered Wildlife

0.90

Wild/scenic Protection

wild/scenic Tributary or

Upstream/Downstream

wild/Scenic Location

0.90 Federal Land Code 103

0.90 Federal Land Code 104

0.90 Federal Land Code 105

0.90 Federal Land Code 106

0.90 Federal Land Code 107

0.90 Federal Land Code 108

0.90 Federal Land Code 198

0.90
0.90

0.90

0.90

$\mathrm{Y} \quad 0.50$

0.90

0.90

0.90

0.90

0.90 
DATE: $08 / 19 / 97$

FERC

Number

Plant Name

NVH0124 SOUTH SIXMILE CANYON

County Name

NYE
PAGE NO: 161

State

Name

\section{SIXMILE CREEK}

NV

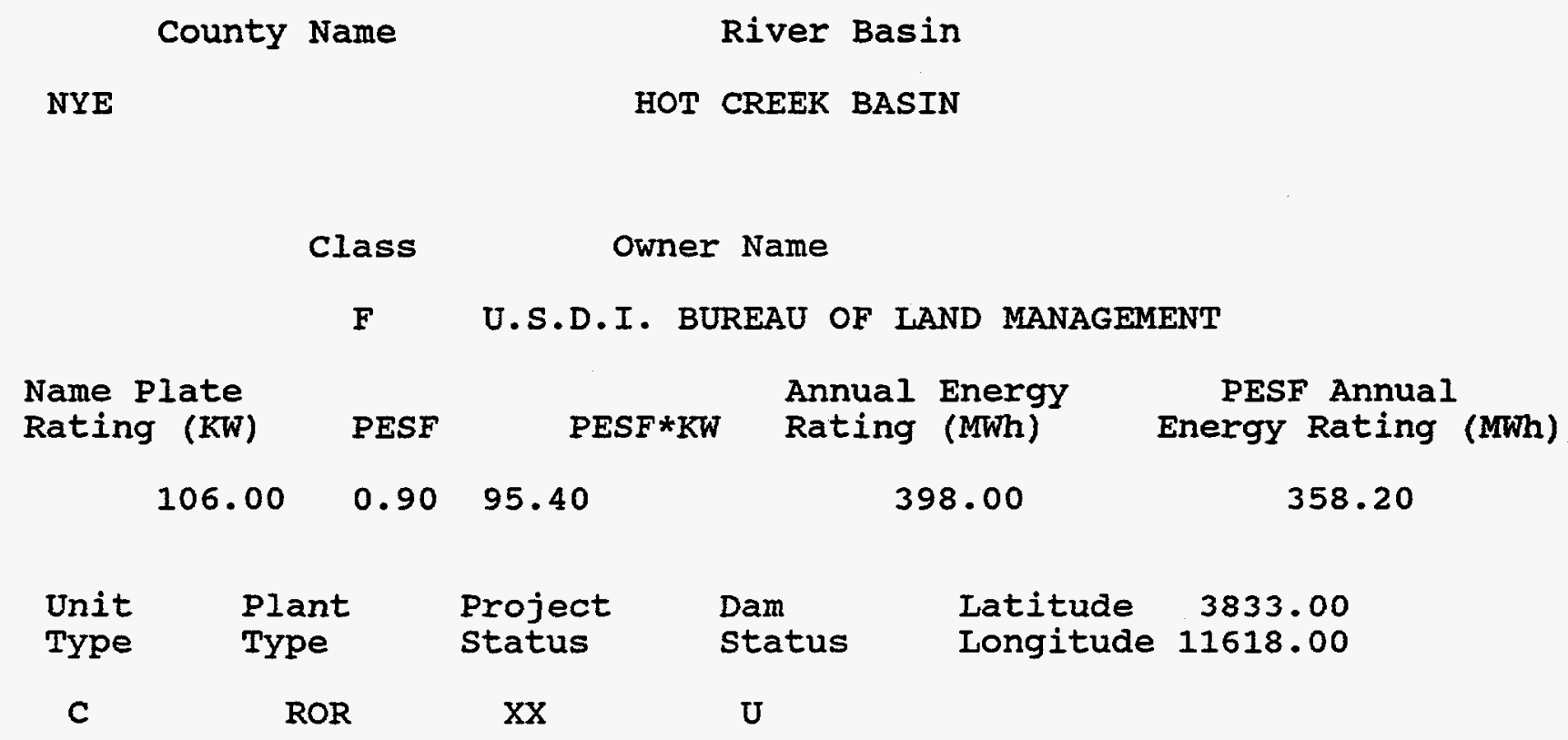

Factor

Exist Prob

Factor

Exist Prob

Wild/Scenic Protection

Wild/Scenic Tributary or

Upstream/Downstream

0.90 Wildife Value

Threatened/Endangered Fish

Threatened/Endangered Wildlife

0.90

wild/Scenic Location

0.90 Federal Land code 103

0.90 Federal Land Code 104

0.90 Federal Land Code 105

0.90 Federal Land Code 106

0.90 Federal Land Code 107

0.90 Federal Land Code 108

0.90 Federal Land code 198

0.90

0.90

0.90

Fish Presence Value

Geologic Value

Historic Value

other Value

Recreation Value

0.90

0.90

0.90

0.90

0.90

0.90

Scenic Value

0.90 
R E S O URCE D A T A B A S E I I S T I N G

DATE: $08 / 19 / 97$

PAGE NO: 162

FERC

Number

Plant Name

Stream

State

NVH0125 CARSON CITY

ASH CANYON CREEK

Name

NV

county Name

River Basin

CARSON CITY

CARSON RIVER BASIN

Class Owner Name

R UNKNOWN

Name Plate

Rating ( $\mathrm{KW}$ )

PESF

Annual Energy

PESF*KW

Rating (MWh)

PESF Annual

244.00

$0.90 \quad 219.60$

890.00

Energy Rating (MWh)

801

\begin{tabular}{|c|c|c|c|c|}
\hline $\begin{array}{l}\text { Unit } \\
\text { Type }\end{array}$ & $\begin{array}{l}\text { Plant } \\
\text { Type }\end{array}$ & $\begin{array}{l}\text { Project } \\
\text { status }\end{array}$ & $\begin{array}{l}\text { Dam } \\
\text { status }\end{array}$ & $\begin{array}{lr}\text { Latitude } & 3911.00 \\
\text { Longitude } & 11948.00\end{array}$ \\
\hline C & ROR & $\mathrm{XX}$ & U & \\
\hline
\end{tabular}

Factor

Exist Prob

Factor

Exist Prob

wild/Scenic Protection

wild/Scenic Tributary or

0.90 Wildlife Value Threatened/Endangered Fish

0.90

Upstream/Downstream Threatened/Endangered Wildlife

0.90

wild/Scenic Location

Cultural value

Fish Presence Value

$\begin{array}{ll}0.90 & \text { Federal Land Code } 103 \\ 0.90 & \text { Federal Land Code } 104\end{array}$

0.90

0.90 Federal Land Code 104

0.90

0.90 Federal Land Code 106

0.90

Geologic Value

0.90

0.90 Federal Land Code 107

0.90

Historic Value

0.90

Federal Land Code 108

0.90

Other Value

0.90 Federal Land Code 198

0.90

Recreation value

0.90

0.90 
DATE: $08 / 19 / 97$

FERC

Number

Plant Name

NVH0126 UNIONVILLE \#2

County Name

PERSHING
PAGE NO: 163

State

Name

INDIAN CREEK

NV
River Basin

BUENA VISTA BASIN

Class Owner Name

C UNKNOWN

\begin{tabular}{|c|c|c|c|c|c|c|}
\hline \multirow[t]{2}{*}{$\begin{array}{l}\text { Name } P \\
\text { Rating }\end{array}$} & $\begin{array}{l}\text { late } \\
(\mathrm{KW})\end{array}$ & PESF & $\mathrm{PESF} * \mathrm{KW}$ & \multicolumn{2}{|c|}{$\begin{array}{l}\text { Annual Energy } \\
\text { Rating (MWh) }\end{array}$} & $\begin{array}{l}\text { PESF Annual } \\
\text { Energy Rating (MWh) }\end{array}$ \\
\hline & 43.00 & 0.90 & 38.70 & \multicolumn{2}{|c|}{180.00} & 162 \\
\hline $\begin{array}{l}\text { Unit } \\
\text { Type }\end{array}$ & $\begin{array}{l}\text { Pl } \\
\text { TYl }\end{array}$ & & $\begin{array}{l}\text { Project } \\
\text { Status }\end{array}$ & $\begin{array}{l}\text { Dam } \\
\text { Status }\end{array}$ & $\begin{array}{l}\text { Latitude } \\
\text { Longitude }\end{array}$ & $\begin{array}{r}4023.00 \\
11807.00\end{array}$ \\
\hline C & \multicolumn{2}{|c|}{ ROR } & $x x$ & \multicolumn{2}{|l|}{ U } & \\
\hline
\end{tabular}

Factor

Exist Prob

0.90

Wild/Scenic Protection

Wild/Scenic Tributary or Upstream/Downstream wild/Scenic Location Cultural Value Fish Presence Value Geologic Value Historic Value other Value Recreation Value Scenic Value

0.90

0.90
Factor

Wildlife Value

Threatened/Endangered Fish Threatened/Endangered Wildlife

0.90 Federal Land Code 103

0.90 Federal Land Code 104

0.90 Federal Land Code 105

0.90 Federal Land Code 106

0.90 Federal Land Code 107

0.90 Federal Land Code 108
Exist Prob

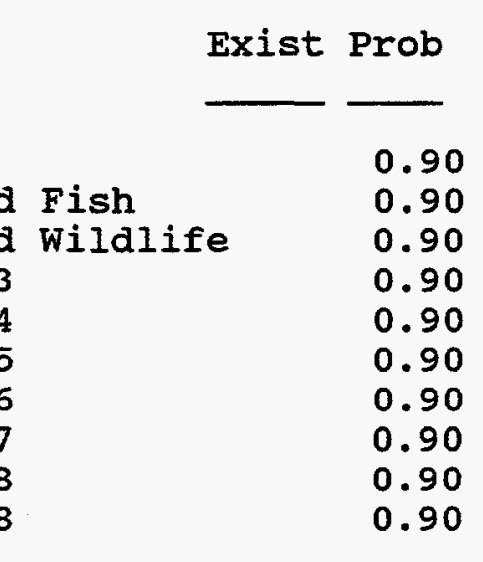

0.90

0.90

0.90

0.90

0.90

0.90

0.90
Federal Land Code 198 
RESOURCE D A T A B A S E I I S T I N G

DATE: $08 / 19 / 97$

PAGE NO: 164

FERC

Number

Plant Name

Stream

State

NVH0127 UNIONVILLE \#3

COTTONWOOD CREEK

Name

NV

County Name

PERSHING
River Basin

BUENA VISTA BASIN
Class Owner Name

C UNKNOWN

Name Plate

Rating (KW)

PESF

Annual Energy

PESF*KW

Rating (MWh)

PESF Annual

$43.00 \quad 0.90 \quad 38.70$

180.00

162

\begin{tabular}{|c|c|c|c|c|}
\hline $\begin{array}{l}\text { Unit } \\
\text { Type }\end{array}$ & $\begin{array}{l}\text { Plant } \\
\text { Type }\end{array}$ & $\begin{array}{l}\text { Project } \\
\text { Status }\end{array}$ & $\begin{array}{l}\text { Dam } \\
\text { status }\end{array}$ & $\begin{array}{lr}\text { Latitude } & 4024.00 \\
\text { Longitude } & 11807.00\end{array}$ \\
\hline C & ROR & $X X$ & $\mathrm{U}$ & \\
\hline
\end{tabular}

Factor

Exist Prob

Factor

Exist Prob

wild/Scenic Protection

wild/Scenic Tributary or

0.90 Wildlife Value

Threatened/Endangered Fish

Upstream/Downstream Threatened/Endangered Wildlife

0.90

0.90

0.90 Federal Land Code 103

0.90

wild/Scenic Location

0.90 Federal Land code 104

0.90

0.90 Federal Land code 105

0.90

Fish Presence Value

0.90 Federal Land Code 106

0.90

0.90 Federal Land Code 107

0.90

Historic Value

0.90 Federal Land code 108

0.90

other value

Recreation value

0.90

Federal Land Code 198

0.90

Scenic Value

0.90

0.90 
R E S O U R C E

DATE: $08 / 19 / 97$
D A T A B A S E

L I S T I N G

PAGE NO: 165

FERC

Number

Plant Name

Stream

state

Name

NVH0128 UNIONVILLE \#I

County Name

PERSHING

BUENA VISTA CREEK

NV

BUENA VISTA BASIN

class Owner Name

R UNKNOWN

\begin{tabular}{|c|c|c|c|c|c|c|}
\hline \multicolumn{2}{|c|}{ Name Plate } & PESF & $P E S F * K W$ & $\begin{array}{l}\text { Annu } \\
\text { Rati }\end{array}$ & $\begin{array}{ll}1 & \text { Energy } \\
g & \text { (MWh) }\end{array}$ & $\begin{array}{l}\text { PESF Annual } \\
\text { Energy Rating (MWh) }\end{array}$ \\
\hline & 79.00 & 0.90 & 71.10 & & 311.00 & 279.90 \\
\hline $\begin{array}{l}\text { Unit } \\
\text { Type }\end{array}$ & $\begin{array}{l}\text { Pl } \\
\text { Tyl }\end{array}$ & & $\begin{array}{l}\text { Project } \\
\text { status }\end{array}$ & $\begin{array}{l}\text { Dam } \\
\text { status }\end{array}$ & $\begin{array}{l}\text { Latitude } \\
\text { Longitude }\end{array}$ & $\begin{array}{r}4027.00 \\
11807.00\end{array}$ \\
\hline C & & & $x x$ & $U$ & & \\
\hline
\end{tabular}

Factor

Exist Prob

0.90

Wild/Scenic Protection

wild/Scenic Tributary or Upstream/ Downstream wild/Scenic Location Cultural value Fish Presence Value

Geologic Value

Historic Value

other Value

Recreation Value

Scenic Value

0.90

\section{Factor}

Exist Prob

wildlife Value

Threatened/Endangered Fish Threatened/Endangered Wildlife

0.90 Federal Land Code 103

0.90 Federal Land Code 104

0.90 Federal Land Code 105

0.90 Federal Land Code 106

0.90 Federal Land Code 107

0.90 Federal Land Code 108

0.90 Federal Land Code 198
0.90

0.90

0.90

0.90

0.90

0.90

0.90

0.90

0.90

0.90 
RE SOU R C E D A TABASE L I S T IN G

DATE: $08 / 19 / 97$

PAGE NO: 166

FERC

Number

Plant Name

NVH0129 BASQUE PLANT

county Name

PERSHING

Class Owner Name

C UNKNOWN

Name Plate

Rating (KW)

PESF

PESF*KW

Annual Energy

$94.00 \quad 0.90 \quad 84.60$

River Basin

MIDDLEGATE BASIN
354.00

Rating (MWh)

$$
354.00
$$

State

Name

NV
PESF Annual Energy Rating (MWh)
318.60

$\begin{array}{cccll}\text { Unit } & \text { Plant } & \text { Project } & \text { Dam } & \text { Latitude } \\ \text { Type } & \text { Type } & \text { Status } & \text { Status } & \text { Longitude } 11743.00 \\ \text { C } & \text { ROR } & \text { XX } & \text { U } & \end{array}$

Factor

Exist Prob

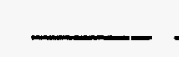

Wild/Scenic Protection

wild/Scenic Tributary or Upstream/Downstream wild/Scenic Location Cultural value Fish Presence Value Geologic Value Historic value other Value Recreation value Scenic Value
Factor

Exist Prob

0.90 Wildlife Value Threatened/Endangered Fish Threatened/Endangered Wildlife

0.90 Federal Land Code 103

0.90 Federal Land Code 104

0.90 Federal Land Code 105

0.90 Federal Land Code 106

0.90 Federal Land Code 107

0.90 Federal Land Code 108

0.90 Federal Land Code 198
0.90

0.90

0.90

0.90

0.90

0.90

0.90

0.90

0.90

0.90 
DATE: $08 / 19 / 97$

FERC

Number

NVH0133 DIAMOND PEAK PLANT

County Name

WASHOE
PAGE NO: 167

state

Name

INCLINE CREEK

NV

\section{Class Owner Name}

R UNKNOWN

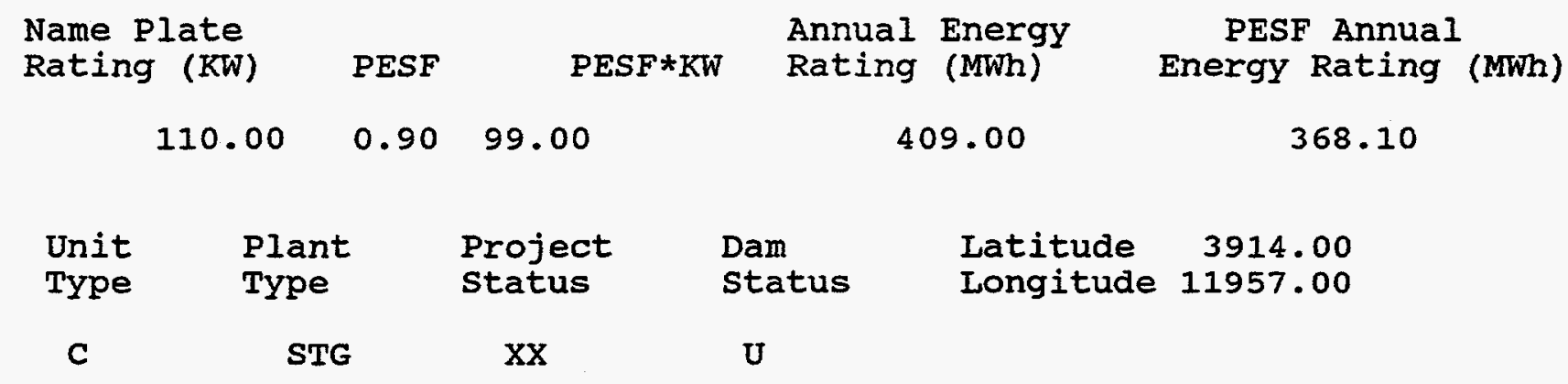

Factor

Wild/Scenic Protection

wild/Scenic Tributary or

Upstream/Downstream

wild/Scenic Location

Cultural value

Fish Presence Value

Geologic Value

Historic Value

other Value

Recreation Value

Scenic Value
Exist Prob

Factor

Exist Prob

\subsection{Wildife Value}

Threatened/Endangered Fish

Threatened/Endangered Wildlife

0.90 Federal Iand Code 103

0.90 Federal Land Code 104

0.90 Federal Land Code 105

0.90 Federal Land Code 106

0.90 Federal Land Code 107

0.90 Federal Land Code 108

0.90 Federal Land Code 198

0.90 
R E S O U R C E

DATE: $08 / 19 / 97$
D A T A B A S E

P

FERC

Number

Plant Name

NVH0134 GREY POWER PLANT

County Name

WASHOE

Class

R UNKNOWN

Name Plate

Rating ( $K W$ )

PESF

PESF*KW

Annual Energy

189.00

0.90

170.10

Rating (MWh)

703.00

River Basin

TRUCKEE RIVER BASIN

PAGE NO: 168

stream

State

Name

NV
PESF Annual Energy Rating (MWh)

\begin{tabular}{|c|c|c|c|c|}
\hline $\begin{array}{l}\text { Unit } \\
\text { Type }\end{array}$ & $\begin{array}{l}\text { Plant } \\
\text { Type }\end{array}$ & $\begin{array}{l}\text { Project } \\
\text { Status }\end{array}$ & $\begin{array}{l}\text { Dam } \\
\text { status }\end{array}$ & $\begin{array}{lr}\text { Latitude } & 3919.00 \\
\text { Longitude } & 12000.00\end{array}$ \\
\hline C & STG & $\mathrm{xX}$ & $\mathbf{U}$ & \\
\hline
\end{tabular}

Factor

Exist Prob

0.90 Wildlife Value Threatened/Endangered $F$ ish Threatened/Endangered Wildlife

0.90 Federal Land code 103

0.90 Federal Land Code 104

0.90 Federal Land code 105

0.90 Federal Land code 106

0.90 Federal Land Code 107

0.90 Federal Land Code 108

0.90 Federal Land code 198
Exist Prob

0.90

0.90

0.90

0.90

0.90

0.90

0.90

0.90

0.90

0.90

Recreation value

0.90 
DATE: $08 / 19 / 97$

PAGE NO: 169

FERC

Number

Plant Name

stream

State

NVH0136 MOUNT ROSE PLANT WEST

DEEP CANYON

Name

County Name

River Basin

WASHOE

TRUCKEE RIVER BASIN

Class Owner Name

C UNKNOWN

$\begin{array}{rrrccc}\begin{array}{l}\text { Name Plate } \\ \text { Rating (KW) }\end{array} & \text { PESF } & \text { PESF*KW } & \begin{array}{c}\text { Annual Energy } \\ \text { Rating (MWh) }\end{array} & \begin{array}{c}\text { PESF Annual } \\ \text { Energy Rating (MWh) }\end{array} \\ 427.00 & 0.50 & 213.50 & 1587.00 & 793.50\end{array}$

$\begin{array}{llllr}\text { Unit } & \text { Plant } & \text { Project } & \text { Dam } & \text { Latitude } \\ \text { Type } & \text { Type } & \text { Status } & \text { Status } & \text { Longitude } 12000.000\end{array}$

C STG $\mathrm{XX} \quad \mathrm{U}$

Factor

Exist Prob

Factor

Exist Prob

wild/Scenic Protection

wild/Scenic Tributary or

0.90 Wildlife Value

0.90

Threatened/Endangered Fish

0.90

Upstream/ Downstream Threatened/Endangered Wildlife

0.90

wild/Scenic Location

0.90 Federal Land Code 103

0.90 Federal Land Code 104

0.90

cultural value

0.90 Federal Land Code 105

0.50

Fish Presence Value

0.90 Federal Land code 106

0.90

Geologic Value

0.90 Federal Land Code 107

0.90

Historic Value

0.90 Federal Land Code 108

0.90

other Value

Recreation Value

0.90

Federal Land Code 198

0.90

Scenic Value

0.90

0.90 
FERC

Number

Plant Name

NVH0137 UNNAMED PLANT

County Name

WASHOE

Class

$\mathbf{R}$

IINKNOWN

Name Plate Rating (KW)

305.00

PESF

PESF*KW

Annual Energy

Rating (MWh)

1113.00

$0.90 \quad 274.50$

Plant

Type

Project

status

C

ROR

Dam
status

Latitude

Longitude 11957.00

$\mathrm{XX}$

U
State Name

NV
PESF Annual Energy Rating (MWh)

\author{
Factor \\ wild/Scenic Protection \\ wild/Scenic Tributary or \\ Upstream/Downstream \\ wild/Scenic Location \\ Cultural value \\ Fish Presence Value \\ Geologic Value \\ Historic Value \\ other Value \\ Recreation Value \\ Scenic Value
}

Exist Prob

\begin{tabular}{cc} 
Exist Prob & \multicolumn{2}{c}{ Factor } \\
\cline { 3 - 3 } 0.90 & $\begin{array}{l}\text { Wildlife Value } \\
\text { Threatened/Endangered Fish } \\
\text { Threatened/Endangered Wildlife }\end{array}$ \\
0.90 & Federal Land Code 103 \\
0.90 & Federal Land Code 104 \\
0.90 & Federal Land Code 105 \\
0.90 & Federal Land Code 106 \\
0.90 & Federal Land Code 107 \\
0.90 & Federal Land Code 108 \\
0.90 & Federal Land Code 198 \\
0.90 &
\end{tabular}

Exist Prob

0.90

0.90

0.90

0.90

0.90

0.90

0.90

0.90

0.90

0.90 
R E S O U R C E

DATE: $08 / 19 / 97$

FERC

Number

Plant Name

NVH0138 BIG INDIAN PLANT

County Name

WHITE PINE

Class Owner Name

C UNKNOWN
PAGE NO: 171

State

Name

BIG INDIAN CREEK

NV

\section{stream}

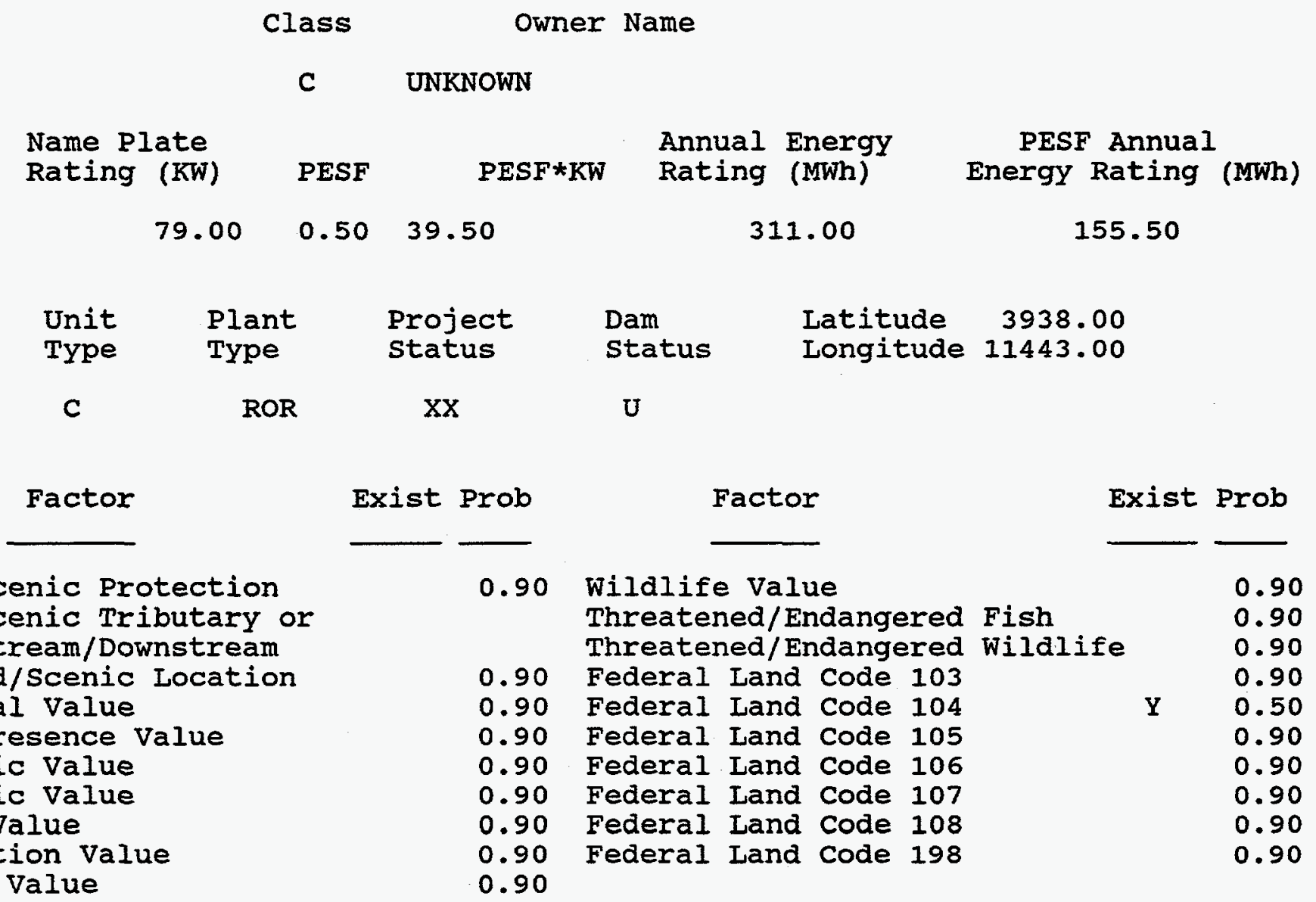

Wild/scenic Protection

Wild/Scenic Tributary or

Upstream/Downstream

wild/Scenic Location

Cultural Value

Fish Presence Value

Geologic Value

Historic Value

other Value

Recreation value

Scenic Value
River Basin

STEPTOE BASIN

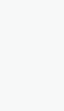



R E S O U R C E
D A T A B A S E
L I S T I N G

DATE: $08 / 19 / 97$

PAGE NO: 172

FERC

Number

Plant Name

stream

State

Name

NVH0139 KALAMAZOO PLANT

KALAMAZOO CREEK

NV

county Name

WHITE PINE
River Basin

GREAT SALT LAKE BASIN
Class Owner Name

F UNKNOWN

Name Plate

Rating (KW)

PESF

Annual Energy

PESF*KW

Rating (MWh)

PESF Annual Energy Rating (MWh)

$$
211.00 \quad 0.50 \quad 105.50
$$

797.00

398.50

\begin{tabular}{|c|c|c|c|c|}
\hline $\begin{array}{l}\text { Unit } \\
\text { Type }\end{array}$ & $\begin{array}{l}\text { Plant } \\
\text { Type }\end{array}$ & $\begin{array}{l}\text { Project } \\
\text { Status }\end{array}$ & $\begin{array}{l}\text { Dam } \\
\text { status }\end{array}$ & $\begin{array}{lr}\text { Latitude } & 3933.50 \\
\text { Longitude } & 11433.00\end{array}$ \\
\hline C & ROR & $\mathrm{xX}$ & $\mathrm{U}$ & \\
\hline
\end{tabular}

Factor

Wild/Scenic Protection

Wild/Scenic Tributary or Upstream/Downstream wild/Scenic Location Cultural Value Fish Presence Value Geologic Value Historic Value other Value

Recreation Value

Scenic value
Exist Prob

0.90 Wildlife Value Threatened/Endangered Fish Threatened/Endangered Wildlife

0.90 Federal Land Code 103

0.90 Federal Land Code 104

0.90 Federal Land code 105

0.90 Federal Land Code 106

0.90 Federal Land Code 107

0.90 Federal Land Code 108

0.90 Federal Land Code 198
Exist Prob

0.90

0.90

0.90

0.90

Y

0.50

0.90

0.90

0.90

0.90

0.90 

R E S O U R C E
D A T A B A S E
I I S T I N G

DATE: $08 / 19 / 97$

PAGE NO: 173

FERC

Number

Plant Name

stream

state

NVHO142 BIG WASH PIANT

BIG WASH CREEK

Name

county Name

River Basin

WHITE PINE

GREAT SALT LAKE BASIN

Class Owner Name

C UNKNOWN

Name Plate

Rating (KW)

Annual Energy

PESF

Rating (MWh)

PESF Annual

185.00

$0.50 \quad 92.50$

681.00

$\begin{array}{lllll}\text { Unit } & \text { Plant } & \text { Project } & \text { Dam } & \text { Latitude } 3853.00 \\ \text { Type } & \text { Type } & \text { Status } & \text { Status } & \text { Longitude 11407.00 }\end{array}$

C

ROR

$\mathrm{XX}$

U

Factor

Wild/Scenic Protection

Wild/Scenic Tributary or

Upstream/Downstream

wild/Scenic Location

Cultural Value

Fish Presence Value

Geologic Value

Historic Value

other value

Recreation Value

Scenic value
Exist Prob

0.90 Wildlife Value Threatened/Endangered Fish Threatened/Endangered wildlife

0.90 Federal Land Code 103

0.90 Federal Land Code 104

0.90 Federal Land Code 105

0.90 Federal Land Code 106

0.90 Federal Land Code 107

0.90 Federal Land Code 108

0.90 Federal Land Code 198

0.90
NV
340.50
Energy Rating (MWh)




\section{RE SOU R C E D A T A B A S E I I S T I N G}

DATE: $08 / 19 / 97$

PAGE NO: 174

FERC

Number

Plant Name

Stream

State

Name

NVHO143 SNAKE PLANT

SNAKE CREEK

NV

county Name

WHITE PINE
River Basin

GREAT SALT IAKE BASIN

\section{Class Owner Name}

C UNKNOWN

Name Plate

Rating ( $\mathrm{KW}$ )

PESF

Annual Energy

Rating (MWh)

609.00

Latitude $\quad 3855.00$

Unit

Plant

Project

status

Dam

status

Longitude 11408.00
C

ROR

$\mathrm{xx}$

U

PESF Annual Energy Rating (MWh)
Factor

Wild/Scenic Protection

Wild/Scenic Tributary or

Upstream/ Downstream

wild/Scenic Location

Cultural value

Fish Presence Value

Geologic Value

Historic Value

other Value

Recreation value

Scenic Value
Exist Prob

0.90

Wildlife Value Threatened/Endangered Fish Threatened/Endangered Wildlife

0.90 Federal Land Code 103

0.90 Federal Land Code 104

0.90 Federal Land Code 105

0.90 Federal Land code 106

0.90 Federal Land Code 107

0.90 Federal Land Code 108

0.90 Federal Land Code 198
Exist Prob

0.90

0.90

0.90

0.90

$Y$

0.50

0.90

0.90

0.90

0.90

0.90 
R E S O UR C E

DATE: $08 / 19 / 97$
D A T A B A S E

L I $S I N G$

FERC

Number

Plant Name

PAGE NO: 175

NVHO144 PRIOR TO PRESTON PLANT

County Name

WHITE PINE

River Basin

COLORADO RIVER BASIN

State

Name

NV

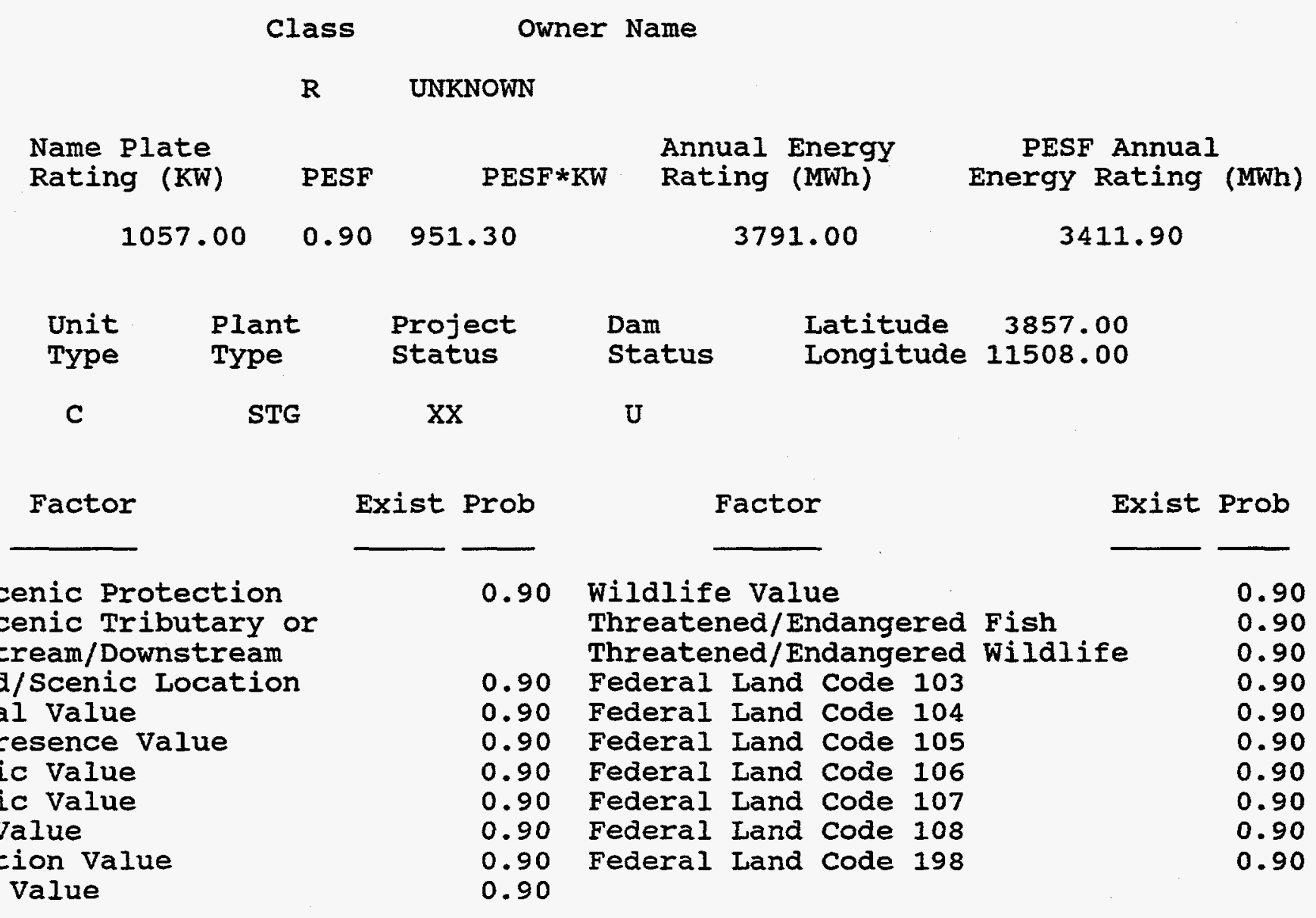

wild/Scenic Protection

Wild/Scenic Tributary or

Upstream/Downstream

wild/Scenic Location

Cultural Value

Fish Presence Value

WHITE RIVER

stream

Geologic Value

Historic Value

other Value

Recreation Value

Scenic Value

Exist Prob

Factor

Exist Prob

0.90

0.90

0.90

0.90

0.90

0.90

0.90

0.90

0.90

Federal Land Code 108

0.90 
FERC

Number

Plant Name

NVU0001 PATSVILLE

County Name

ELKO

Class

c UNKNOWN

Name Plate

Rating (KW)

PESF

PESF*KW

Annual Energy

1600.00

$0.10 \quad 160.00$

OWYHEE RIVER

stream

state

Name

NV
River Basin

SNAKE RIVER BASIN 290

\begin{tabular}{|c|c|c|c|c|}
\hline $\begin{array}{l}\text { Unit } \\
\text { Type }\end{array}$ & $\begin{array}{l}\text { Plant } \\
\text { Type }\end{array}$ & $\begin{array}{l}\text { Project } \\
\text { status }\end{array}$ & $\begin{array}{l}\text { Dam } \\
\text { status }\end{array}$ & $\begin{array}{lr}\text { Latitude } & 4148.00 \\
\text { Longitude } & 11557.30\end{array}$ \\
\hline C & STG & $x X$ & $\mathrm{U}$ & \\
\hline
\end{tabular}

Factor

Wild/Scenic Protection

Wild/scenic Tributary or

Upstream/Downstream

wild/Scenic Location

Cultural value

Fish Presence Value

Geologic Value

Historic Value

other Value

Recreation value

Scenic Value
Exist Prob

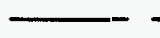

$\begin{array}{cc} & 0.90 \\ & \\ & \\ & 0.90 \\ & 0.90 \\ Y & 0.25 \\ & 0.90 \\ & 0.90 \\ Y & 0.50 \\ Y & 0.25 \\ & 0.90\end{array}$

\section{Factor}

Exist Prob

Wildife Value

Threatened/Endangered Fish Threatened/Endangered Wildlife

Federal Land Code 103

Federal Land Code 104

Federal Land Code 105

Federal Land Code 106

Federal Land code 107

Federal Land Code 108

Federal Land Code 198
PESF Annual Energy Rating (MWh) 

R E S O U R C E
D A T A B A S E
I I S T I N G

DATE: $08 / 19 / 97$

PAGE NO: 177

FERC

Number

Plant Name

NVU0002 SKULI CREEK

County Name

EIKO

Class

C UNKNOWN

Name Plate

Rating (KW)

PESF

Annual Energy

Rating (MWh)

6400.00

$0.10 \quad 340.00$

Project

status

XX

Exist Prob

Factor

Type

STG

Factor

Wild/Scenic Protection

Wild/Scenic Tributary or

Upstream/Downstream

wild/Scenic Location

Cultural Value

Fish Presence Value

Geologic Value

Historic Value

other value

Recreation Value

Scenic Value
OWYHEE RIVER

River Basin

SNAKE RIVER BASIN $\begin{array}{llr}\text { Dam } & \text { Latitude } & 4155.00 \\ \text { Status } & \text { Longitude } & 11604.00\end{array}$

$\mathbf{U}$

$\begin{array}{cclll} & & & & \\ & 0.90 & \begin{array}{l}\text { Wildife Value } \\ \text { Threatened/Endangered Fish }\end{array} & 0.90 \\ & & \text { Threatened/Endangered Wildlife } & 0.90 \\ & 0.90 & \text { Federal Land Code 103 } & 0.90 \\ & 0.90 & \text { Federal Land Code 104 } & 0.90 \\ \text { Y } & 0.25 & \text { Federal Land Code 105 } & 0.90 \\ & 0.90 & \text { Federal Land Code 106 } & 0.90 \\ & 0.90 & \text { Federal Land Code 107 } & 0.90 \\ & 0.90 & \text { Federal Land Code 108 } & 0.90 \\ \text { Y } & 0.25 & \text { Federal Land Code 198 } & 0.90 \\ & 0.90 & & \end{array}$

state

Name

NV
PESF Annual

Energy Rating (MWh)

640 
RES O U C E D A T A B S E L I S T I N

DATE: $08 / 19 / 97$

PAGE NO: 178

FERC

Number

Plant Name

Stream

State

NVU0017 DEVIL'S GATE RESERVOIR

NORTH FORK HUMBOLDT RIVER

Name

MVOO17 DEVIL'S GAIE RESERVOIR

County Name

River Basin

ELKO

HUMBOLDT RIVER BASIN

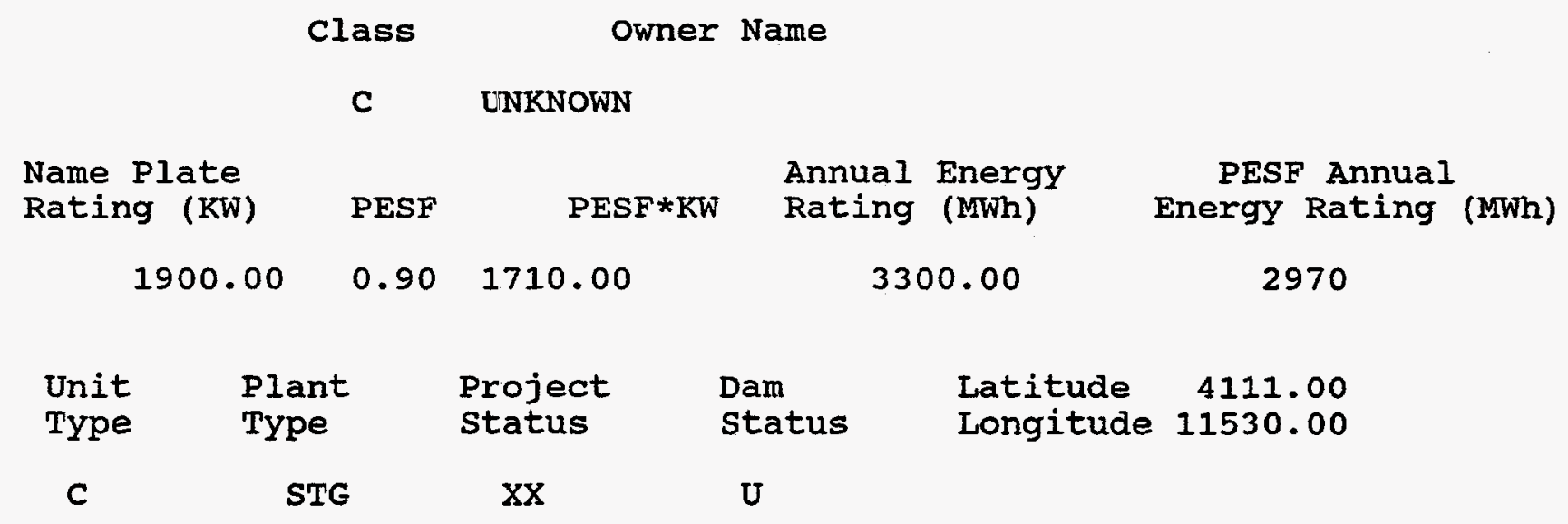

Factor

Exist Prob

Factor

Exist Prob

Wild/Scenic Protection

wild/Scenic Tributary or

0.90 Wildlife Value

Threatened/Endangered Fish

Upstream/Downstream

wild/Scenic Location

Cultural value

Fish Presence Value

Geologic Value

Historic Value

Other Value

Recreation value

Scenic value
Threatened/Endangered Wildlife

0.90 Federal Land code 103

0.90 Federal Land Code 104

0.90 Federal Land code 105

0.90 Federal Land Code 106

0.90 Federal Land Code 107

0.90 Federal Land code 108

0.90 Federal Land Code 198

0.90
NV 
DATE: $08 / 19 / 97$

FERC

Number

Plant Name

NVUO018 EUREKA DAM

County Name

LYON
PAGE NO: 179

state

Name

NV
CARSON RIVER

River Basin

CARSON RIVER BASIN

\section{Class Owner Name}

C UNKNOWN

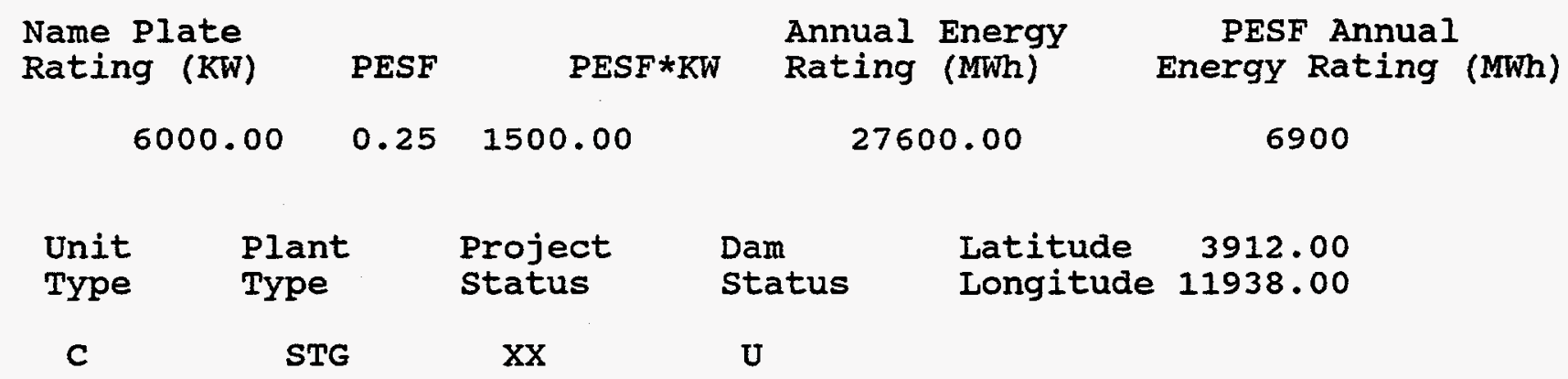

Factor

Wild/Scenic Protection

wild/Scenic Tributary or

Upstream/Downstream

wild/Scenic Location

Cultural Value

Fish Presence Value

Geologic Value

Historic Value

other Value

Recreation Value

Scenic Value
Exist Prob

Factor

\subsection{Wildlife Value}

Threatened/Endangered Fish

Threatened/Endangered Wildlife

0.90 Federal Land Code 103

0.90 Federal Land Code 104

0.90 Federal Land Code 105

0.90 Federal Land Code 106

0.90 Federal Land Code 107

Y $\quad 0.50$ Federal Land code 108

$Y \quad 0.25$

0.90
Exist Prob

\begin{tabular}{ll} 
& \multicolumn{2}{c}{ Exist Prob } \\
& \\
Fish & 0.90 \\
Wildife & 0.90 \\
& 0.90 \\
& 0.90 \\
& 0.90 \\
& 0.90 \\
& 0.90 \\
& 0.90 \\
& 0.90 \\
& 0.90
\end{tabular}

0.90

0.90

0.90

0.90

0.90

0.90 

R E S O U R C E
D A T A B A S E
I I S I N G

DATE : $08 / 19 / 97$

PAGE NO: 180

FERC

Number

Plant Name

stream

State

NVUO020 HOYE CANYON

WEST WALKER RIVER

Name

county Name

River Basin

DOUGLAS

WALKER LAKE BASIN

NV

Class Owner Name

R WALKER RIVER IRRIGATION DISTRICT

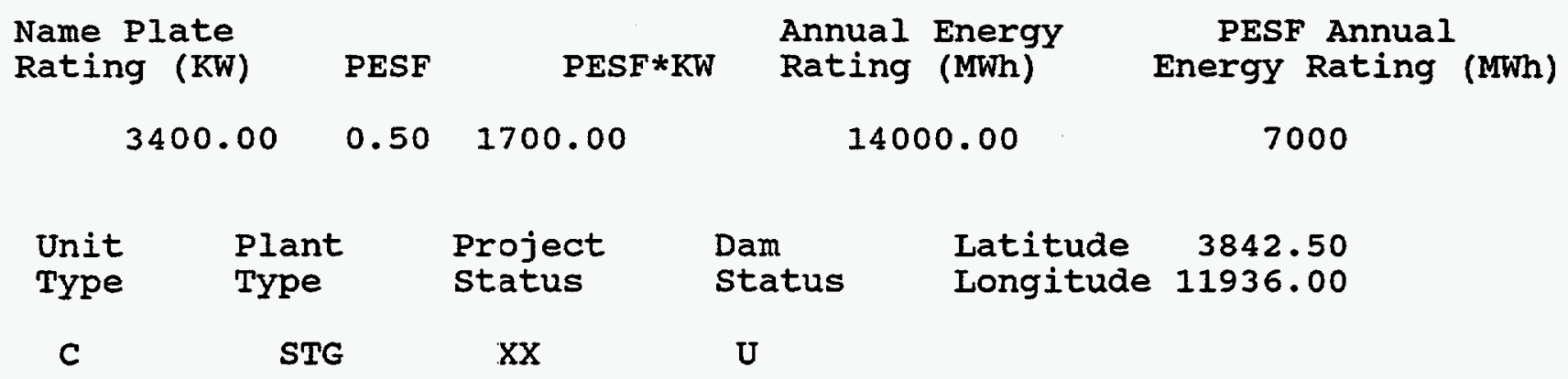

Factor

Exist Prob

Factor

Exist Prob

wild/Scenic Protection

wild/Scenic Tributary or

0.90 Wildlife Value Threatened/Endangered Fish

0.90

0.90

Upstream/Downstream wild/Scenic Location

$\begin{array}{ll}0.90 & \text { Federal Land Code } 103 \\ 0.90 & \text { Federal Land Code } 104\end{array}$

0.90

0.90 Federal Land Code 105

0.90

Cultural Value

Fish Presence Value

0.90 Federal Land Code 106

0.90

Geologic Value

0.90 Federal Land Code 107

0.90

Historic Value

other Value

Recreation value

0.50

Federal Land Code 108

0.90

$\mathrm{Y}$

0.90

Federal Land Code 198

0.90

0.90

0.90

0.90

Scenic Value 
DATE : $08 / 19 / 97$

FERC

Number

NVU0023 KEYSTONE RESERVOIR

County Name

WHITE PINE

Class Owner Name

C UNKNOWN state

Name

GLEASON CREEK

NV

stream

V

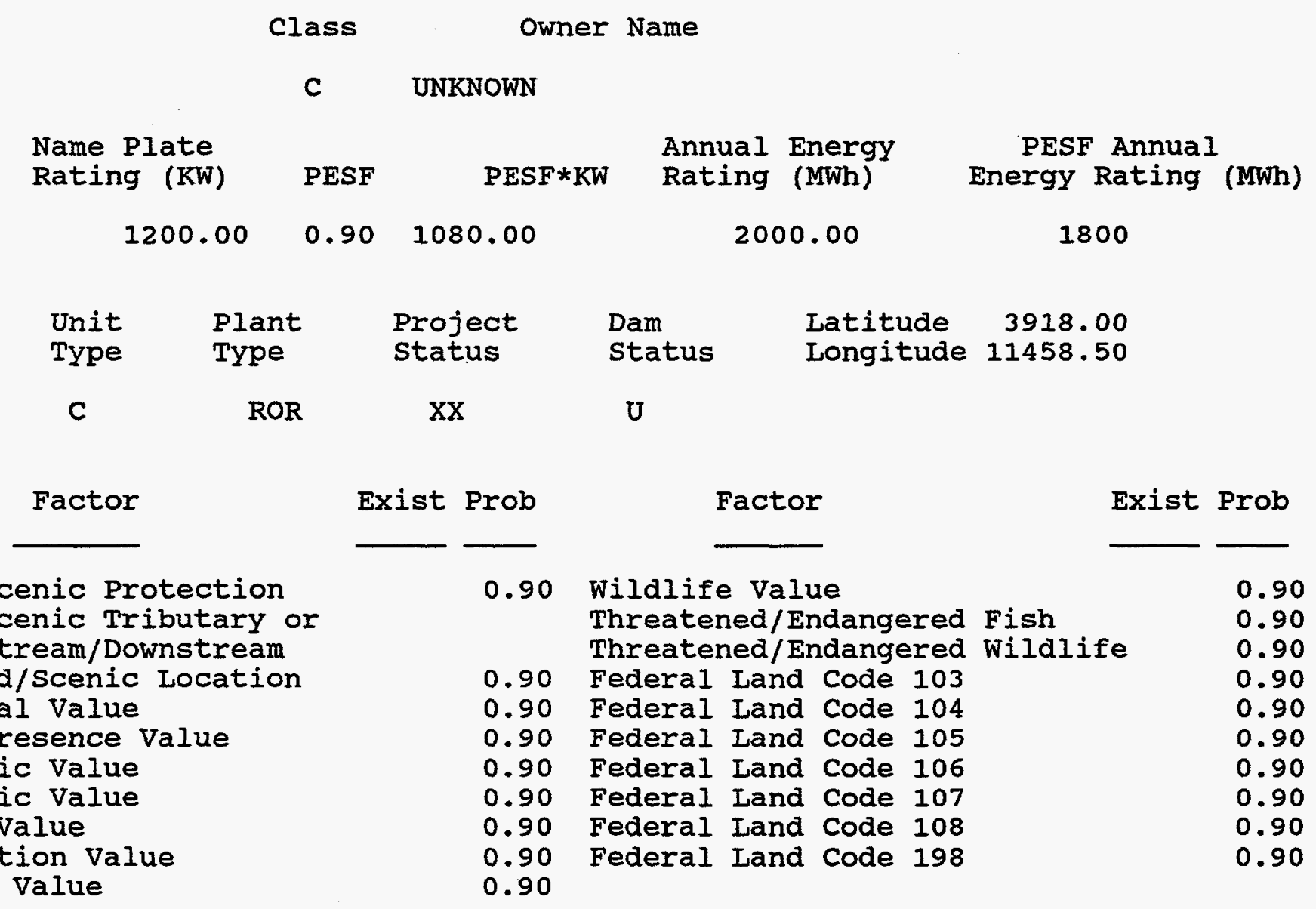

Wild/Scenic Protection

Wild/Scenic Tributary or

Upstream/Downstream wild/Scenic Location

Cultural value

Fish Presence Value

Geologic Value

Historic Value

other Value

Recreation Value

Scenic Value

River Basin

STEPTOE BASIN

PAGE NO: 181 


\section{Appendix E}

\section{List of 201 Additional Sites}


- LIST OFF STREAM, PLANT MM, DAMSTATUS, CNTY NM, BASIN NM, LAT U, LONG U, OWNER NM STREAM

ADOBE CREEK

ALLERMAN CANAL

ALLERMAN CANAL

ALLERMAN CANAL

BEAVER DAM WASH

BEAVER DAM HASH

BIG SPRINGS

BILK CREEK

BILLY SHAW SLOUGH

BLACK CANYON

BLUE JACKET CREEK

BOG HOT SPRING

BOULDER SPRING

BRUNEAU RIVER

BUCK RAKE JACK CREEK

BUFFALO SLOUGH

BULL CREEK

BULL RUN CREEK

BULL RUN CREEK

BULL RUN CREEK

BUSHEE CREEK

CALAGHAN CREEK

CARICO CREEK

CARSON RIVER

CARSON RIVER

CARSON RIVER

CARSON RIVER

CARSON RIVER

CATNIP CREEK

CAVE CREEK

CHIMNEY CREEK

CIRCLE CREEK

CIRCLE CREEK

COLD CREEK

COLEMAN CREEK

COLORADO RIVER

COTTONWOOD CREEK

COTTONWOOD CREEK

COTTONHOOD CREEK

CRITTENDEN CREEK

CRYSTAL \& BRADFORD SPRINGS

CURRANT CREEK
PLANT_NM DAMSTATUS CNTY_NM

CRESTVIEH DAM

ALLERMAN \#1 RESERVOIR WO DOUGLAS

ALLERMAN \#2

ALLERMAN \#3 RESERVOIR WO DOUGLAS

BEAVER DAM U CLARK

SCHROEDER RESERVOIR WO LINCOLN

IZZENHOCD RESERVOIR WO LANDER

LOWER BILK CREEK RESERVOIR WO HUMBOLDT

COYOTE HOLE RESERVOIR WO ELKO

MILK RANCH RESERVOIR U HASHOE

COLUMBIA RANCH

BOG HOT RESERVOIR

WO HO ELKO

PALISADES \#4

DEWEY-PARKER

BULL CREEK \#3

MAGGIE

RAWHIDE RESERVOIR

WILSON RESERVOIR

BUSHEE CREEK RESERVOIR HO PERSHING

HUNT FIELD DAM

CARICO LAKE

HARMON RESERVOIR

LAHONTAN DAM

OLLIE'S POND

SAGOUSPIE DIVERSION

SHECKLER RESERVOIR

CATNIP RESERVOIR

CAVE CREEK RESERVOIR

CHIMNEY CREEK RESERVOIR

GROUNDHOG RESERVOIR

JOSEPHINE RESERVOIR

COLD CREEK RESERVOIR

COLEMAN RESERVOIR

HOOVER DAM

COTTONHOOD \#1

COTTONWOOD \#2

FLY RESERVOIR

CRITTENDEN RESERVOIR

CRYSTAL LAKE

MANZONIE
BASIN_NM

HUMBOLDT RIVER BASIN

CARSON RIVER BASIN

CARSON RIVER BASIN

CARSON RIVER BASIN

COLORADO RIVER BASIN

COLORADO RIVER BASIN

HUMBOLDT RIVER BASIN

BLACK ROCK DESERT BASII

SNAKE RIVER BASIN

HONEY LAKE BASIN

SNAKE RIVER BASIN

CONTINENTAL LAKE BASIN

SUPRISE BASIN

SNAKE RIVER BASIN

HUMBOLDT RIVER BASIN

SMOKE CREEK DESERT BASIN

RAILROAD BASIN

SNAKE RIVER BASIN

SNAKE RIVER BASIN

SNAKE RIVER BASIN

HUMBOLDT RIVER BASIN

GRASS BASIN

HUMBOLDT RIVER BASIN

CARSON RIVER BASIN

CARSON RIVER BASIN

CARSON RIVER BASIN

CARSON RIVER BASIN

CARSON RIVER BASIN

SUPRISE BASIN

STEPTOE BASIN

SNAKE RIVER BASIN

SNAKE RIVER BASIN

SNAKE RIVER BASIN

NEWARK BASIN

BIG LAKE BASIN

COLORADO RIVER BASIN

TRUCKEE RIVER BASIN

TRUCKEE RIVER BASIN

BLACK ROCK DESERT BASIN

GREAT SALT LAKE BASIN

DEATH VALLEY BASIN

RAILROAD BASIN
LAT_U LONG_U OWNER_MM

4051.0011549 .00 CHARLES CHESTER

3857.0011943 .00 DANGBERG HOLDINGS LLC

3858.0011943 .00 DANGBERG HOLDINGS LLC

3859.0011943 .00 DANGBERG HOLDINGS LLC

3727.0011402 .00 MESQUITE FARMSTEAD MATER ASSOCIATIO

3731.0011404 .00 NEVADA DEPARTMENT OF CONSERVATION

4055.00 11654.00 25 CORPORATION

4136.0011826 .00 C2 CATTLE COMPANY

4126.0011614 .00 PETAN COMPANY

$3958.0011949 .00 \mathrm{~W}$. DALTON AND JUANITA LARUE

4140.0011605 .00 AGRI BEEF COMPANY

$4155.0011847 .00 \mathrm{~J}$. L. COONAN

4121.0011945 .00 HOME CAMP L.\& L. COMPANY, INC

4135.0011530 .00 ROBERT PRUNTY

4033.00 11613.00 USDI BUREAU OF RECLAMATION

4036.0011946 .00 ESPIL SHEEP COMPANY

3901.0011538 .00 D. A. WITTS

4138.00 11604.00 AGRI BEEF COMPANY

4143.0011620 .00 PETAN COMPANY

4141.0011621 .00 PETAN COMPANY

4021.0011737 .00 ROBERT \& DIANA VESCO

3941.0011650 .00 MOLLY F. KNUDTSEN

4008.0011653 .00 HENRY FILIPPINI

3929.0011838 .00 TRUCKEE-CARSON IRRIGATION DISTRICI

3928.0011904 .00 TRUCKEE-CARSON IRRIGATION DISTRICT

3931.0011842 .00 TRUCKEE-CARSON IRRIGATION DISTRICT

3932.0011844 .00 TRUCKEE-CARSON IRRIGATION DISTRICT

3926.0011854 .00 TRUCKEE-CARSON IRRIGATION DISTRICT

4155.0011928 .00 USDI FISH AND HILDLIFE SERVICE

3911.0011442 .00 NEVADA DEPARTMENT OF CONSERVATION

4143.0011633 .00 ROARING SPRINGS ASSOCIATES

4156.00 11620.00 PETAN COMPANY

4154.0011627 .00 PETAN COMPANY

3951.0011545 .00 BALDARELLI

$4159.0011947 .00 \mathrm{D}$. L. NICOL

3601.0011444 .00 USDI BUREAU OF RECLAMATION

3935.00 11953.00 SALVADOR URRITIA

3936.0011952 .00 SALVADOR URRITIA

$4052.0011920 .00 \mathrm{~J}$. J. CASEY

4132.0011410 .00 WALKER-WINECUP-GAMBLE INCORPORATED

3625.0011620 .00 SPRING MEADOWS, INCORPORATED

3847.0011523 .00 USDA FOREST SERVICE 
DAVIS AND OPHIR CREEKS DEADMAN'S CANYON CREEK DEEP CREEK

DEER CREEK

DENAY CREEK

DESERT CREEK

DESERT CREEK

DOG CREEK

DORSEY CREEK

DRY CREEK

DRY CREEK

DUCK CREEK

DUCK CREEK

DUCK FLAT WASH

EAGLE GULCH

EAGLE VALLEY CREEK

EAST FORK CAARSON RIVER

EAST FORK CARSON RIVER

EAST FORK OWYHEE RIVER

EAST FORK WALKER RIVER EFFLUENT

EFFLUENT

EFFLUENT STORAGE DAM

EIGHT MILE CREEK

ELDORADO CANYON CREEK

EVANS CERRK

EVANS CREEK

FISH ANO BADGER CREEKS

FRANKTOWN CREEK

FRANKTOWN CREEK

FRANKTOWN CREEK

GEYSER \& NORTH CREEKS

GOLD CREEK

GRASS VALLEY CREEK

HENDERSON CREEK

HOLY LAKE CREEK

HORSE CREEK

HOT CREEK

HUMBOLDT RIVER

HUMBOLDT RIVER

HUMBOLOT RIVER

HUMBOLDT RIVER

HUMBOLDT RIVER

ILL. IPAH CREEK

INDIAN CREEK
DAVIS CREEK DAM GREIL RESERVOIR DEEP CREEK RESERVOIR

DELONG RESERVOIR

TONKIN RESERVOIR

NUTI AND SON RESERVOIR

NUTI RESERVOIR

DOG CREEK DAM

DORSEY CREEX RESERVOIR

DRY CREEK RESERVOIR

SPANISH FLAT RESERVOIR

BASSET LAKE

DUCK LAKE

DISABEL RESERVOIR

POWERS DAM

EAGLE VALLLY RESERVOIR

SETTLEMEYER RANCH

WILSLEF MILL.

WILDHORSE RESERVOIR

STALL BROTHERS MILL.

BUCKEYE CREEK

ROUNDHILL EFFLUENT

CARSON CITY WASTEWATER

EIGHT MILE PLANT

ELDORADO CANYON DAM

HERMAN DAM

TOWLE RESERVOIR

SWAN LAKE

CLIFF RESERVOIR

HOBART RESERVOIR

RED HOUSE DIVERSION

GEYSER DAM \#2

SUNFLOWER RESERVOIR

GRASS VALLEY RESERVOIR

J D RESERVOIR

SEIVER LAKE RESERVOIR

CHISHOLM

GIBBS RANCH RESERVOIR

PITT DAM

ROGERS DAM

STALL DAM

UPPER PITT-TAYLOR RESER.

UPPER SLAVEN

ILLIPAH POND

MUD LAKE

$\begin{array}{ll}\text { WO } & \text { WASHOE } \\ \text { WO } & \text { WASHOE } \\ \text { WO } & \text { ELKO } \\ \text { WO } & \text { HUMBOLDT } \\ \text { WO } & \text { EUREKA } \\ \text { WO } & \text { LYON } \\ \text { WO } & \text { LYON } \\ \text { U } & \text { WASHOE } \\ \text { WO } & \text { ELKO } \\ \text { WO } & \text { ELKO } \\ \text { WO } & \text { WASHOE } \\ \text { WO } & \text { WHITE PINE } \\ \text { WO } & \text { WHITE PINE } \\ \text { U } & \text { WASHOE } \\ \text { U } & \text { WASHOE } \\ \text { WO } & \text { LINCOLN } \\ \text { W } & \text { DOUULAS } \\ \text { W } & \text { DOUGLAS } \\ \text { WO } & \text { ELKO } \\ \text { WO } & \text { LYON } \\ \text { WO } & \text { DOUGLAS } \\ \text { WO } & \text { DOUGLAS } \\ \text { WO } & \text { CARSON CITY } \\ \text { W } & \text { WHITE PINE } \\ \text { WO } & \text { LYON } \\ \text { WO } & \text { WASHOE } \\ \text { WO } & \text { WHITE PINE } \\ \text { U } & \text { WASHOE } \\ \text { WO } & \text { WASHOE } \\ \text { WO } & \text { WASHOE } \\ \text { WO } & \text { WASHOE } \\ \text { WO } & \text { WASHOE } \\ \text { WO } & \text { WHITE PINE } \\ \text { WO } & \text { ELKO } \\ \text { WO } & \text { WASHOE } \\ \text { WO } & \text { EUREKA } \\ \text { WO } & \text { WASHOE } \\ \text { U } & \text { CHURCHILL } \\ \text { U } & \text { ELKO } \\ \text { WO } & \text { PERSHING } \\ \text { WERSHING } \\ \text { HUMBOLDT } \\ \text { PERSHING } \\ \text { WANDER } \\ \text { WO } & \\ \text { WOSH } \\ \text { WO }\end{array}$

TRUCKEE RIVER BASIN TRUCKEE RIVER BASIN SNAKE RIVER BASIN

BLACK ROCK DESERT BASIN

HUMBOLDT RIVER BASIN

WALKER LAKE BASIN

WALKER LAKE BASIN

TRUCKEE RIVER BASIN

HUMBOLDT RIVER BASIN

SNAKE RIVER BASIN

HONEY LAKE BASIN

STEPTOE BASIN

STEPTOE BASIN

SUPRISE BASIN

SUPRISE BASIN

COLORADO RIVER BASIN

CARSON RIVER DASTH

CARSON RIVER BASIN

SNAKE RIVER BASIN

WALKER RIVER BASIN

CARSON RIVER BASIN

LAKE TAHOE BASIH

CARSON RIVER BASIN

SPRING BASIN

CARSON RIVER BASIN

TRUCKEE RIVER BASIN

TRUCKEE RIVER BASIN

LONG BASIN

TRUCKEE RIVER BASIN

TRUCKEE RIVER BASIN

TRUCKEE RIVER BASIN

SPRING BASIN

SNAKE RIVER BASIN

BLACK ROCK DESERT BASIN

HUMBOLDT RIVER BASIN

LONG BASIN

DIXIE VALLEY BASIN

HUMBOLOT RIVER BASIN

HUMBOLDT RIVER BASIN

HUMBOLDT RIVER BASIN

HUMBOLDT RIVER BASIN

HUMBOLDT RIVER BASIN

HUMBOLDT RIVER BASIN

JAKES BASIN

CARSON RIVER BASIN
3918.00 11950.00 WASHOE COUNTY PARKS

3915.0011945 .00 JAMES P. ROSS

4135.0011619 .00 ROARING SPRINGS ASSOCIATION

4123.00 11831.00 DELONG RANCHES INCORPORATED

3955.0011625 .00 FRANK PAXTON

3846.0011919 .00 SIX-N RANCH, INCORPORATED

3847.0011919 .00 SIX-N RANCH, INCORPORATED SIERRA PACIFIC POWER COMPANY

4104.00 11549.00 FRANK HOOPER

4148.0011614 .00 PETAN COMPANY

$4000.0011947 .00 \mathrm{H}$. DALTON AND JUANITA LARUE

3928.0011451 .00 KENNECOTT CORPORATION

3928.0011442 .00 KENNECOTT CORPORATION

4057.00 11941.00 USDI BUREAU OF LAND MANAGEMENT

4135.0011955 .00 H. J. POWERS

3801.0011412 .00 NEVADA DEPARTMENT OF CONSERVATION

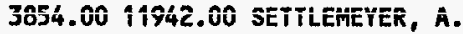

3855.0011944 .00 WILSLEF, THOMAS $\mathrm{N}$.

4141.0011550 .00 DUCK VALLEY INDIAN COLONY

3826.0011906 .00 STALL BROTHERS

3859.0011940 .00 DOUGLAS COUNTY SEWER IMP. DIST. \#1

3859.0011955 .00 DOUGLAS COUNTY SEWER IMP. DIST. \#1

3910.00 11940.00 CARSON CITY UTILITIES

3923.00 11422.00 GEORGE ELDRIDGE AND SON, INC.

3913.00 11934.00 DAVADA INCORPORATED

3933.0011950 .00 WASHOE COUNTY PARKS

3928.0011950 .00 CEASAR GASPARI

4152.0011929 .00 USDI FISH AND WILDLIFE SERVICE

3915.0011949 .00 DONALD A. CLIFF

3912.00 11952.00 NEVADA DEPARTMENT OF ADMINISTRATION

3912.00 11952.00 NEVADA DEPARTMENT OF ADMINISTRATION

3840.0011438 .00 D. A. WITTS

4145.00 11543.00 RAYMOND MENDIVE

4106.0011930 .00 HOLLAND LIVESTOCK COMPANY

4005.0011620 .00 DIAMOND LAND AND CATTLE COMPANY

4146.0011953 .00 LEE HUTCHENS

3932.0011801 .00 HARLES CHISHOLM

4134.0011511 .00 W. H. GIBBS

4014.00 11825.00 PERSHING COUNTY WATER CONSV. DIST.

4012.00 11827.00 PERSHING COUNTY WATER CONSV. DIST.

4058.00 11726.00 NEVADA FIRST CORPORATION

4038.00 11821.00 PERSHING COUNTY WATER CONSV. DIST.

4040.00 11644.00 USDI BUREAU OF RECLAMATION

3920.0011523 .00 NEVADA DEPARTMENT OF CONSERVATION 3851.0011944 .00 AQUADUCT 1 
IOWA CREEK

JAKE'S WASH

JAKES CREEK

JAKES CREEX

JAKES CREEK

JAMES CREEK

JOHN DAY CREEK

JONES CANYON CREEK

JUMBO CREEK

LEIDY CREEK

LIITLE HIGH ROCK CREEK

LITTLE HIGH ROCK CREEK

LITTLE NEGRO CREEK

LOST CREEK

MACAFEE CREEK

MARLETTE CREEK

MASON CREEK

MASSACRE CREEK

MC FAUL CREEK

MCAFEE CREEK

MILL CREEK

MILL CREEK

MILL CREEK

MINE DEWATERING

MOORMAN SPRINGS WASH

MOSQUI TO CREEK

MUD MEADOW CREEK

MUD MEADOWS CREEK

MUD MEADOWS CREEK

MUD MEADOWS CREEK

MUDDY RIVER

MUSGROVE CREEK

NEGRO CREEK

NEGRO CREEK

NEGRO CREEK

NEH YEAR LAKE

NIAGARA CREEK

NICHOLAS SPRING

NORTH CANYON CREEK

NORTH CREEK

PASQUALE SPRING

PETERSON CREEK

QUINN RIVER

RAGAN CREEK

REESE RIVER
LOWA CREEK RESERVOIR

PRESTON DAM

LANOER

A. H. AGEE RESERVOIR

BOIES RESERVIOR

DESMOND DAM

JAMES CREEK DIVERSION

JOHW OAY RESERVOIR

JONES CANYON RESERVOIR

JUMBO DAM

FISH LAKE DAM

DENIO CAMP RESERVOIR

LITTLE HIGH ROCK

ELDRIDGE L. N. PLANT

LOST CREEK RESERVOIR

MACAFEE CREEK RESERVOIR

MARLETTE RESERVOIR

DINNER STATION RESERVOIR

CAP JOHNSON RESERVOIR

BOURNE RESERVOIR

MCAFEE

FILIPPINI RANCH RESERVOIR WO

MILL CREEK \#2

MILL. CREEK DAM

MAGGIE CREEK RESERVOIR

DACEY RESERVOIR

HILL DAM \#1

MUD MEADOWS RESERVOIR

PARMAN RESERVOIR

WEISS-VOGEL RESERVOIR

WHEELER RESERVOIR

HONEYBEE RESERVOIR

RANCH DAMS

IVESON DAM

NEGRO CREEK PLANT

HIGGER CREEK RESERVOIR

FROG POND

URIGHT RANCH

NICOLAS MILL

SPOONER LAKE

GEYSER DAM \#5

PASQUALE RESERVOIR

PETERSOH

FORT MCDERMITT RESERVOIR

PUMPERNICKEL RESERVOIR

LEDLIE RESERVOIR
HUMBOLDT RIVER BASIN

COLORADO RIVER BASIN

SNAKE RIVER BASIN

SNAKE RIVER BASIN

HUMBOLDT RIVER BASIN

HUMBOLDT RIVER BASIN

HUMBOLOT RIVER BASIN

TRUCKEE RIVER BASIM

TRUCKEE RIVER BASIN

FISH LAKE BASIII

BLACK ROCK DESERT BASIN

BLACK ROCK DESERT BASI

SPRING BASIN

SUPRISE BASIN

FISH LAKE BASIN

LAKE TAHOE BASIN

HUMBOLDT RIVER BASIN

SUPRISE BASIN

LAKE TAHOE BASIN

HUMBOLDT RIVER BASIM

HUMBOLDT RIVER BASIN

LAKE TAHOE BASIN

SNAKE RIVER BASIN

HUMBOLDT RIVER BASIN

COLORADO RIVER BASIM

SUPRISE BASIN

BLACK ROCK DESERT BASIN

BLACK ROCK DESERT BASIM

BLACK ROCK DESERT BASIN

BLACK ROCK DESERT BASIN

COLORADO RIVER BASIN

TRUCKEE RIVER BASIII

BLACK ROCK DESERT BASII

SPRING BASIN

BLACK ROCK DESERT BASIN

SUPRISE BASIN

SWAKE RIVER BASIN

COLORADO RIVER BASIN

LAKE TAHOE BASIH

SPRING BASIN

HUMBOLOT RIVER BASIN

HUMBOLDT RIVER BASIN

BLACK ROCK DESERT BASIN

HUMBOLDT RIVER BASIN

HUMBOLDT RIVER BASIM
$3949.0011702 .00 P$, INCHAUSPE

2901.0011505 .00 PRESTON IRRIGATION COMPANY

4137.0011453 .00 O. F. BOIES

4136.0011453 .00 BOIES RANCHES

4111.0011704 .00 PACIFIC STATES SAVIKG AMD LOAN

4046.00 11613.00 MEWMONT GOLD COMPANY

4053.0011524 .00 FRANK HOOPER

3943.0011924 .00 USDI BUREAU OF LAND MANAGEMENT

3917.0011944 .00 TANIA MALOFF

3745.0011803 .00 FOUR RENT INCORPORATED

4115.0011929 .00 ROGER AND KATHLEEN DAVIS

4114.0011926 .00 ERNEST MURPHY

3926.0011430 .00 GEORGE ELDRIDGE AND SON, INC.

4104.0011946 .00 GEORGE CHRISTENSEN

3739.0011804 .00 ERNEST $M$. PELLKOFER

3911.0011955 .00 USDA FOREST SERVICE

4106.0011553 .00 FRANK HOOPER

4136.0011944 .00 UNKNOWN

3860.0011956 .00 S H BOURNE 1994 TRUST

4131.0011555 .00 NORTH FORK CATTLE COMPANY

4024.00 11637.00 HENRY FILIPPINI

3914.0011955 .00 INCLINE VILLAGE GENERAL IMP. DIST.

4149.0011559 .00 DINSDALE AND HIEDERBERG

4048.00 11609.00 NEWMONT GOLD COMPANY

3823.0011507 .00 NEVADA DEPARTMENT OF CONSERVATION

$4148.0011951 .00 \mathrm{~W}$. LEININGER

4119.0011910 .00 SUSAN VALLEY RANCH

4110.0011907 .00 SUSAN VALLEY RANCH

4105.0011905 .00 ANDREW \& GROVER JACKSON

4112.0011907 .00 SUSAN VALLEY RAHCH

3632.0011425 .00 NEVADA DIVISION OF WILDLIFE

3913.00 11949.00 LIGHTMIMG "HU RAHCH

3957.0011922 .00 LOU FASCIO

3917.0011424 .00 ELORIDGE, BRENT, ET AL

4657.0011923 .00 LOU FASCIO

41.0011956 .00 FEE RANCH

4123.0011604 .00 JAMES J. URIGHT RANCH, INC.

3855.0011504 .00 ALLRED, HERBERT

3907.0011955 .00 NEVADA STATE PARKS

3841.0011437 .00 D. A. WITTS

4107.0011724 .00 JACK \& IRENE FULLENWTDER

4132.0011555 .00 NORTH FORK CATTLE COMPANY

4159.0011735 .00 FORT MCDERMITT IMDIAN RESERVATION

4041.0011730 .00 ELLISON RANCHING COMPANY

3731.0011710 .00 SILVER CREEK RANCH 
ROBERTS CREEK

ROCK CREEK

RODEO CREEK

ROSE CREEK

ROSE CREEK

ROSS CREEK

S-LINE CANAL

SAND CREEK

SANDSTONE SPRING

SAVONIA CREEK

SHEEP CREEK

SHINGLE CREEK

SMITH CREEK

SHOW CREEK

SPRING CREEK

SPRING VALLEY CREEK

SPRING YALLEY WASH

SQUAW CREEK

STAFFOO CANYON

STATE WATER SUPPLY PIPELINE

STEAMBOAT CREEK

SUGAR CANE SPRINGS

SUNNYSIDE CREEK

THOMAS CREEK

THOMAS CREEK \& EFFLUENT

THOUSAND CREEK

TROUT CREEK

TROUT CREEK

TROY CREEK

TRUCKEE RIVER

TRUCKEE RIVER

TRUCKEE RIVER \& EVANS CREEK

TWIN SPRINGS CREEK

UNDERGROUND WATER

UNNAMED

UNNAMED

UNNAMED

UNNAMED

UNNAMED CREEK

UNNAMED CREEK

UNNAMED SPRING

UNNAMED SPRING

UNMAMED SPRINGS

UNNAMED TRIB. TO

UNNAMED WASH
ROBERTS CREEK RESERVOIR

NELSON RESERVOIR

RODEO CREEK DAM

ROSE CREEK DAM

ROSE CREEK RESERVOIR

ROSS CANYON DAMS

S-LINE RESERVOIR

CARTER RESERVOIR

SPRING MOUNTAIN

SAVONIA

SHEEP CREEK RESERVOIR

SHINGLE PLANT

SMITH CREEK RESERVOIR

ANGLEWORM RANCH

SPRING CREEK RESERVOIR

ECHO CANYON RESERVOIR

SFRINUO VALLEY WASH RES.

SQUAH CREEK RESERVOIR

PALISADES \#3

TANK

PAGNI POND

SUGAR CANE SPRINGS POND

OLD PLACE DIKE \#3

ALEXANDER LAKE

SOUTH TRUCKEE MEADOWS

BIG SPRING RESERVOIR

JAYNES RESERVOIR

HELCOME RESERVOIR

OLD ENGLISH MILL

RENO DIVERSION

WASHOE DIVERSION

LAKE STANLEY

HOME RANCH RESERVOIR

SILVEY RESERVOIR

PRIVATE POND

ROCK SPRING TABLE

ROUND MOUNTAIN RESERVOIR

SAVAL RESERVOIR

HILL DAM \#2

EGBERT RESERVOIR

DAM "C"

LEAR PLANT

PIUTE MEADOWS RESERVOIR
WO EUREKA

HO LANDER

WASHOE

WASHOE

MINERAL

HASHOE

CHURCHILL

WASHOE

CLARK

ELKO

ELKO

WHITE PINE

LANDER

NYE

ELKO

LINCOLN

WHiTE PINE

WASHOE

EUREKA

CARSON CITY

WASHOE

WASHOE

NYE

WASHOE

WASHOE

HUMBOLDT

ELKO

ELKO

NYE

WASHOE

WASHOE

WASHOE

WASHOE

PERSHING

CLARK

HUMBOLDT

ELKO

ELKO

HASHOE

WASHOE

ELKO

ELKO

NYE

HASHOE

HUMBOLDT
KOBEH BASIN

HUMBOLDT RIVER BASIN

TRUCKEE RIVER BASIN

LAKE TAHOE BASIH

WALKER LAKE BASIN

HONEY LAKE BASIN

CARSON RIVER BASIN

SUPRISE BASIN

COLORADO RIVER BASIN

SNAKE RIVER BASIN

SNAKE RIVER BASIN

SPRING BASIN

SMITH CREEK BASIN

RAILROAD BASIN

HUMBOLDT RIVER BASIN

COLORADO RIVER BASIN

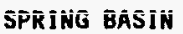

SMOKE CREEK DESERT BASIN

HUMBOLDT RIVER BASIN

CARSON RIVER BASIN

TRUCKEE RIVER BASIN

TRUCKEE RIVER BASIN

COLORADO RIVER BASIN

TRUCKEE RIVER BASIN

TRUCKEE RIVER BASIN

CONTINENTAL LAKE BASIN

HUMBOLDT RIVER BASIN

HUMBOLDT RIVER BASIN

RAILROAD BASIN

TRUCKEE RIVER BASIN

TRUCKEE RIVER BASIN

TRUCKEE RIVER BASIN

TRUCKEE RIVER BASIN

HUMBOLDT RIVER BASIN

COLORADO RIVER BASIN

CONTINENTAL LAKE BASIN

SWAKE RIVER BASIN

HUMBOLDT RIVER BASIN

SUPRISE BASIN

LONG BASIN

HUMBOLDT RIVER BASIN

HUMBOLDT RIVER BASIN

DEATH VALLEY BASIN

TRUCKEE RIVER BASIN

BLACK ROCK DESERT BASIN
3946.0011617 .00 EUREKA LIVESTOCK COMPANY 4031.0011650 .00 JOHN MARVEL

$3949.0011931 .00 \mathrm{H}$. DALTON AND JUANITA LARUE

3916.0011958 .00 WAHSOE COUNTY

3835.0011845 .00 U.S. ARMY

3949.0011956 .00 ROSS CREEK RANCH

3929.0011843 .00 TRUCKEE-CARSON IRRIGATION DISTRICT

$4141.0011956 .00 \mathrm{~J}$. E. BUHYARD

3604.0011528 .00 NEVADA STATE PARKS

4155.0011530 .00 8UCK CREEK RANCH, INC.

4152.0011616 .00 DUCK VALLEY INDIAN COLONY

3959.0011425 .00 HARBECKE, ROBERT L. \& FERN A.

3923.0011738 .00 JACK VAN SICKLE

3846.00 11529.00 DON LANI

4044.00 11534.00 SPRING CREEK ASSOCIATION

3755.0011416 .00 NEVADA DEPARTMENT OF CONSERVATION

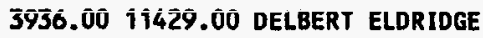

4049.0011932 .00 J. J. CASEY

4031.00 11613.00 USDI BUREAU OF RECLAMATION

3912.0011950 .00 MARLETTE POUER CORPORATION

3920.0011930 .00 DAHLAHI NEVADA CORPORATION

3957.0011948 .00 W. DALTON AND JUANITA LARUE

3825.0011625 .00 NEVADA DEPARTMENT OF CONSERVATION

3928.0011944 .00 RUTH M. BUTLER

3928.00 11945.00 WASHOE COUNTY

$4156.0011910 .00 \mathrm{~J}$. L. COONAN

4105.0011507 .00 FRANK HOOPER

4107.0011506 .00 FRANK HOOPER

3821.0011534 .00 OLD ENGLISH GOLD CORPORATION

3931.0011951 .00 SIERRA PACIFIC POHER COMPANY

3931.0011958 .00 SIERRA PACIFIC POWER COMPANY

3928.00 11949.00 LAKESHORE HOMEOWNER'S ASSOCIATION

3943.00 11930.00 FTY INCORPORATED

4013.0011828 .00 DOROTHY SILVEY

3619.0011538 .00 FIGGINS, JOEL, ET AL

4144.0011931 .00 UNKNOWN

4143.0011615 .00 PETAN COMPANY

4116.0011553 .00 A. GREER EDHARDS, JR.

$4143.0011953 .00 \mathrm{~W}$. LEININGER

4150.0011948 .00 UNKNOWN

4117.0011501 .00 FARNES EGBERT

4117.0011501 .00 FARNES EGBERT

3625.0011616 .00 SPRING MEADOWS INCORPORATED

3929.0011959 .00 LEAR, DAVID

4118.0011856 .00 IRV BROWN 
VIRGIN CREEK

WADE DRAIN

WALKER RIVER

WARM SPRINGS \& COLD CREEKS

WARM SPRINGS CREEK

WET RAVIME WASH

WHISKEY AND LORRIE'S CREEKS

WHITE RIVER

WHITE RIVER

WHITE RIVER

WHITE RIVER

WHITE RIVER

WHITE RIVER

WHITE ROCK CREEK

WILCOX CANYON CREEK

WILCOX CANYON CREEK

WILCOX CANYON CREEK

WILLOW CREEK

WILLOW CREEK

WILLOW CREEK

WILLOW CREEK

WILLOW SPRING

- SET PRINT OFF

$\begin{array}{lll}\text { ANDERSON RANCH } & \text { WO } & \text { WASHOE } \\ \text { BLACK BEAUTY RESERVOIR } & \text { WO } & \text { MINERAL } \\ \text { ALKALI RESERVOIR } & \text { WO } & \text { HUMBOLDT } \\ \text { OLD RIVER DAM } & \text { WO } & \text { CHURCHILL } \\ \text { PERRIN RESERVOIR } & \text { WO } & \text { LYON } \\ \text { HARM SPRINGS } & \text { W } & \text { ELKO } \\ \text { MATLEY DAM } & \text { WO } & \text { HASHOE } \\ \text { WET RAVINE } & \text { WO } & \text { HASHOE } \\ \text { LORRIE'S RESERVOIR } & \text { U } & \text { HASHOE } \\ \text { ADAMS-MCGILL RESERVOIR } & \text { WO } & \text { NYE } \\ \text { COLD SPRINGS } & \text { WO } & \text { NYE } \\ \text { HAY MEADOH DIKE } & \text { WO } & \text { NYE } \\ \text { SUNHYSIDE RESERVOIR } & \text { WO } & \text { LINCOLN } \\ \text { TULE FIELD } & \text { WO } & \text { NYE } \\ \text { UPPER PAHRANAGAT } & \text { WO } & \text { LINCOLN } \\ \text { WHITE ROCK } & \text { U } & \text { ELKO } \\ \text { WILCOX CANYOH DAM \#1 } & \text { WO } & \text { WASHOE } \\ \text { HILCOX CANYOH DAM \#2 } & \text { WO } & \text { WASHOE } \\ \text { HILCOX CANYON DAM \#3 } & \text { WO } & \text { WASHOE } \\ \text { ANGEL LAKE } & \text { WO } & \text { ELKO } \\ \text { BMG WILLOH CREEK RESERVOIR WO } & \text { LANDER } \\ \text { SWINGLE RANCH POND } & \text { WO } & \text { WASHOE } \\ \text { WILLOW CREEK RESERVOIR } & \text { HO } & \text { ELKO } \\ \text { SANDSTONE PLANT } & \text { W } & \text { CLARK }\end{array}$

TRUCKEE RIVER BASIM

WALKER LAKE BASIN

CONTINENTAL LAKE BASIN

CARSON RIVER BASIN

WALKER LAKE BASIN

HUMBOLDT RIVER BASIN

TRUCKEE RIVER BASIN

HONEY LAKE BASIN

TRUCKEE RIVER BASIN

COLORADO RIVER BASIN

COLORADO RIVER BASIN

COLORADO RIVER BASIN

COLORADO RIVER BASIM

COLORADO RIVER BASIN

COLORADO RIVER BASIM

SNAKE RIVER BASIM

TRUCKEE RIVER BASIN

TRUCKEE RIVER BASIN

TRUCKEE RIVER BASIN

HUMBOLDT RIVER BASIN

BUFFALO BASIN

BLACK ROCK DESERT BASIN

HUMBOLDT RIVER BASIN

COLORADO RIVER BASIN
3938.0011955 .00 A. E. ANDERSON

3832.0011842 .00 U.S. ARMY

4138.0011908 .00 HARRY WILSON

3931.0011846 .00 TRUCKEE-CARSON IRRIGATION DISTRICT 3853.0011922 .00 PERRIN \& SONS

4049.00 11523.00 HOOPER, JENNIFER A.. ET AL

3955.0011945 .00 INTERMOUNTAIN LAND COMPANY

3938.0011959 .00 JACK AND JOSEPHINE SHEENEY

$3957.0011949 .00 \mathrm{~W}$. DALTON \& JUANITA LARUE

3822.0011507 .00 NEVADA DEPARTMENT OF CONSERVATION

3740.0011539 .00 NEVADA DEPARTMENT OF CONSERVATION

3820.0011510 .00 NEVADA DEPARTMENT OF CONSERVATION

3822.0011513 .00 MEVADA DEPARTMENT OF CONSERVATION

3819.0011511 .00 NEVADA DEPARTMENT OF CONSERVATION

3711.0011507 .00 USDI FISH AND HILDLIFE SERVICE

4142.0011610 .00 BLUE JACKET MINING COMPANY

3942.0011930 .00 JOHN R. ALBIN

3942.0011931 .00 ARTHUR 1. RELOJ

3942.0011931 .00 CATTLEMEN'S GUARANTEE TITLE COMPANY

4102.0011505 .00 MARSHALL MORGAN

4014.00 11710.00 BATTLE MOUNTAIN GOLD CORPORATION

4108.0011923 .00 MICHAEL B. STEWART

4113.0011632 .00 ELLISON RANCHING COMPANY

3610.0011527 .00 LINCOLN STONE COMPANY 